\title{
Regio- and Stereoselective Cyanotriflation of Alkynes Using
}

\section{Aryl(cyano)iodonium Triflates}

\author{
Xi Wang, and Armido Studer* \\ Institute of Organic Chemistry, University of Münster, Corrensstrasse 40, 48149 \\ Münster, Germany \\ E-mail: studer@uni-muenster.de
}

\section{Table of Contents}

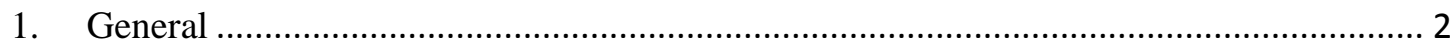

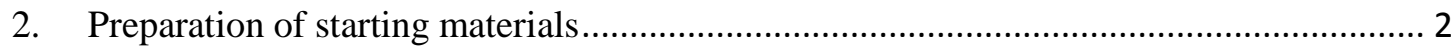

General procedure for the preparation of alkynes 3 from the corresponding aryl iodides

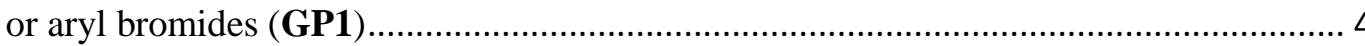

3. Regio- and stereoselective cyanotriflation of alkynes using aryl(cyano)-iodonium

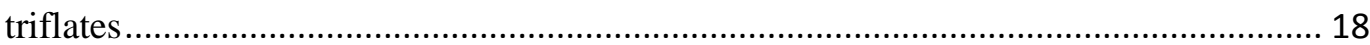

General procedure for cyanotriflation of alkynes (GP2) .......................................... 18

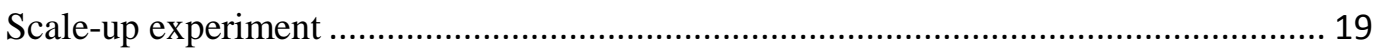

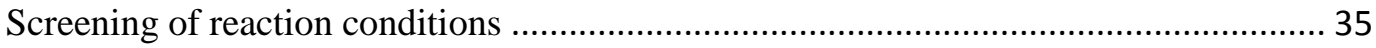

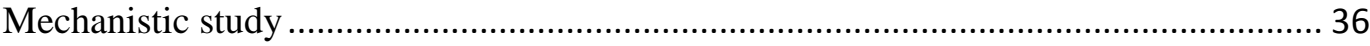

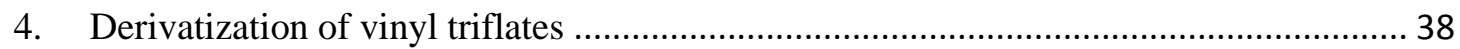

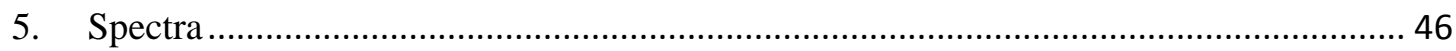

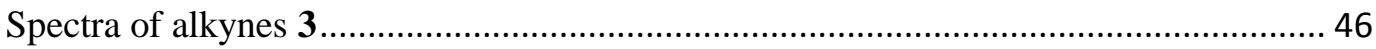

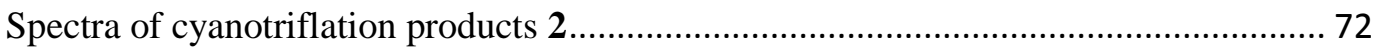

Spectra of products 4-11 in follow-up chemistry ……................................................. 125

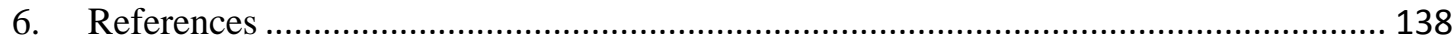




\section{General}

All reactions involving air- or moisture-sensitive reagents or intermediates were carried out in pre-heated glassware under an argon atmosphere using standard Schlenk techniques. THF was freshly distilled from $\mathrm{K}$ under argon. All other solvents and reagents were purified according to standard procedures or were used as received from Alfa Aesar, TCI, Aldrich, Fluka, Acros or ABCR. The alkynes were synthesized according to literature procedures. IR spectra were recorded on a Digilab FTS 4000 with a Specac MKII Golden Gate Single Refelxtion ART System. ${ }^{1} \mathrm{H}$ NMR and ${ }^{13} \mathrm{C}$ NMR spectra were recorded on a DPX 300, AV 400 or DD2 600 at $300 \mathrm{~K}$. Spectra were calibrated relative to solvent's residual proton and carbon chemical shift: $\mathrm{CHCl}_{3}(\delta=$ 7.26 for ${ }^{1} \mathrm{H}$ NMR and $\delta=77.0$ for 13C NMR). TLC was performed using Merck silica gel 60 F-254 plates, detection of compounds with UV light or dipping into a solution of $\mathrm{KMnO}_{4}\left(1.5 \mathrm{~g}\right.$ in $\left.400 \mathrm{~mL} \mathrm{H} \mathrm{H}_{2} \mathrm{O}, 5 \mathrm{~g} \mathrm{NaHCO}_{3}\right)$, followed by heating. Flash column chromatography (FC) was performed using Merck or Fluka silica gel $60(40-63 \mu \mathrm{m})$ applying a pressure of about 0.2 bar. Mass spectra were recorded on a Finnigan MAT 4200S, a Bruker Daltonics Micro Tof, a Waters-Micromass Quatro LCZ (ESI); peaks are given in $m / z$ (\% of basis peak).

\section{Preparation of starting materials}

Aryl(cyano)iodonium triflates $\mathbf{1 a}, \mathbf{1 b}, \mathbf{1 c}$ were prepared according to the previously reported literature procedures. ${ }^{[1]}$

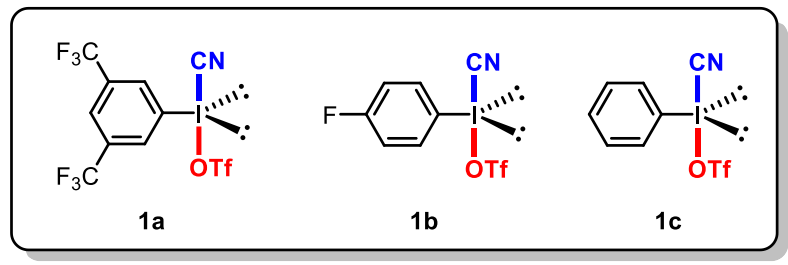

Alkyne 3a is commercially available from Alfa Aesar and was used as received. Alkyne $3 \mathbf{t}$ was prepared according to a previously reported literature procedure. ${ }^{[2]}$ 


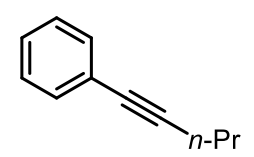

$3 a$

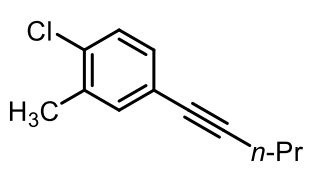

3d<smiles>CCCPC#Cc1ccc(OC(C)=O)cc1</smiles>

$3 g$

$\mathrm{F}_{3} \mathrm{CO}$<smiles>CCCC#Cc1ccc(C)cc1</smiles>

$3 i$<smiles>C=PC#Cc1cccc(C=O)c1</smiles>

$3 m$<smiles>CCCPC#C/C=C/c1ccc(Cl)cc1</smiles>

$3 p$

Pr

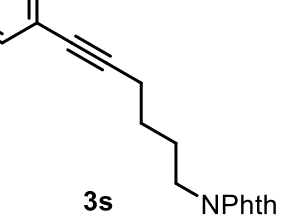

$\mathrm{Br}$<smiles>CC(C)(C)C#Cc1ccc(F)cc1</smiles>

$3 v$<smiles>Cc1ccc(C#CC(C)C)cc1</smiles>

$3 b$

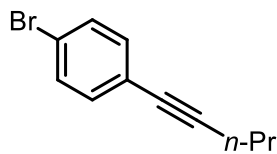

$3 e$

PhthN<smiles>CCCPC#Cc1ccc(C)cc1</smiles>

3h<smiles>C=PC#Cc1ccc(SC(F)(F)F)cc1</smiles>

3k

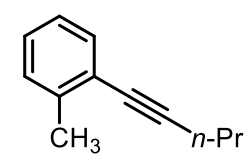

$3 n$

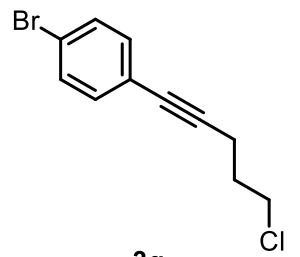

$3 q$<smiles>CC#Cc1ccc(Br)cc1</smiles>

$3 t$

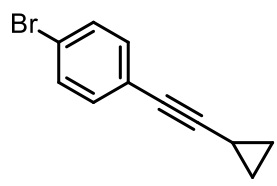

$3 w$

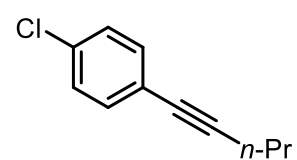

$3 c$

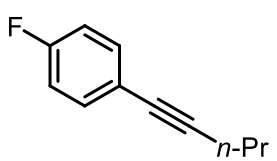

$3 f$

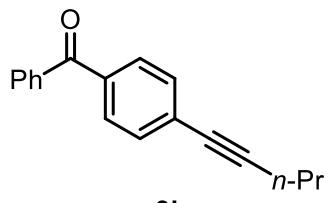

$3 i$

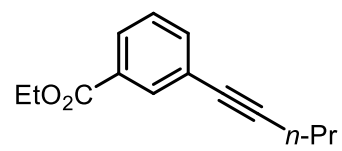

3I

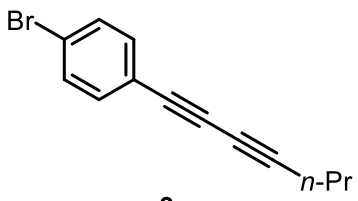

30

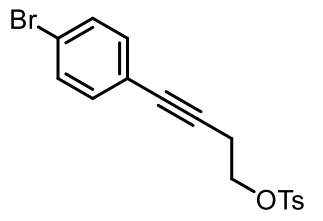

$3 r$

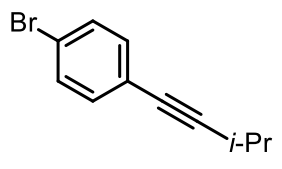

$3 u$<smiles>Brc1ccc(C#CC2CCCC2)cc1</smiles>

$3 x$<smiles>Brc1ccc(C#CC2CCCCC2)cc1</smiles>

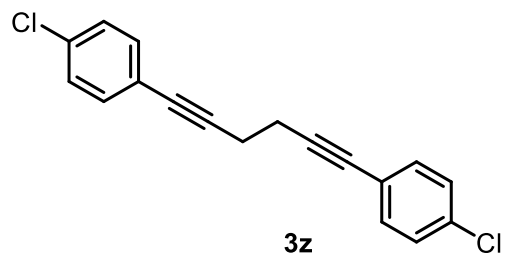


General procedure for the preparation of alkynes 3 from the corresponding aryl iodides or aryl bromides (GP1)

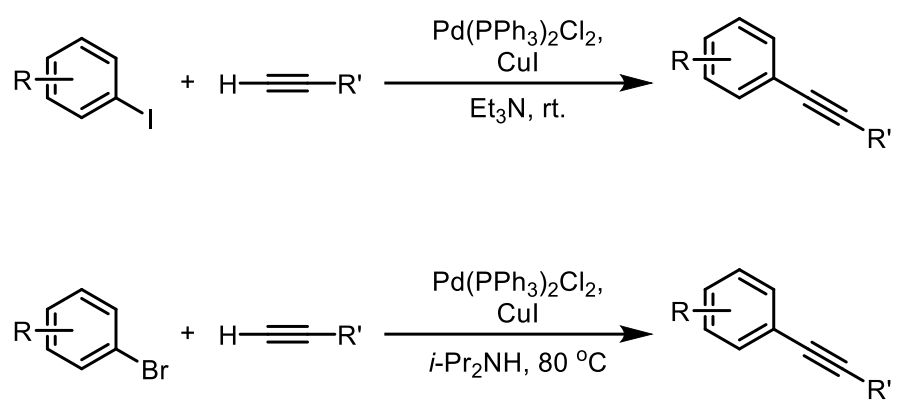

A flame-dried Schlenk-flask equipped with a magnetic stir bar was charged with bis(triphenylphosphine)palladium(II) dichloride, copper(I) iodide, aryl iodide or aryl bromide, sealed with a septum, and degassed by alternating vacuum evacuation and argon backfilling (three times) before triethylamine or diisopropylamine was added. The corresponding terminal alkyne was added to the resulting suspension subsequently. The reaction mixture was then stirred at room temperature or $80^{\circ} \mathrm{C}$ for 12 hours. After the reaction was complete, the reaction mixture was diluted with $\mathrm{Et}_{2} \mathrm{O}(30 \mathrm{~mL})$ and filtrated through a small pad of silica gel. The solvent was removed under reduced pressure with the aid of a rotary evaporator and the crude residue was purified by a silica gel column chromatography to give the corresponding pure alkynes $\mathbf{3}$.

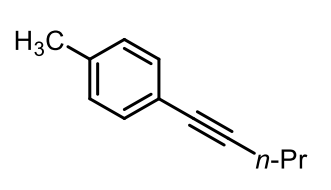

1-Methyl-4-(pent-1-yn-1-yl)benzene (3b): The title compound was prepared according to general procedure (GP1) with $\mathrm{Pd}\left(\mathrm{PPh}_{3}\right)_{2} \mathrm{Cl}_{2}(70.2 \mathrm{mg}, 0.100 \mathrm{mmol}, 1.0 \mathrm{~mol} \%), \mathrm{CuI}(38.1 \mathrm{mg}$, $0.200 \mathrm{mmol}, 2.0 \mathrm{~mol} \%$ ), 1-iodo-4-methylbenzene (2.180 g, $10.00 \mathrm{mmol}, 1.0$ equiv), and pent-1-yne $\left(0.817 \mathrm{~g}, 12.0 \mathrm{mmol}, 1.2\right.$ equiv) in $\mathrm{Et}_{3} \mathrm{~N}(10 \mathrm{~mL})$ at room temperature for 12 hours. Purification via silica gel chromatography (Pentane) gave the desired product $\mathbf{3 b}$ as a yellow oil in 94\% yield (1.482 g). TLC $\mathbf{R}_{\mathrm{f}}=0.85$ (Pentane); ${ }^{1} \mathbf{H}$ NMR $\left(300 \mathrm{MHz}, \mathrm{CDCl}_{3}, 300 \mathrm{~K}\right): \delta(\mathrm{ppm})=7.21(\mathrm{~d}, J=8.1 \mathrm{~Hz}, 2 \mathrm{H}), 7.00(\mathrm{~d}, J=8.1 \mathrm{~Hz}, 2 \mathrm{H})$, $2.29(\mathrm{t}, J=7.0 \mathrm{~Hz}, 2 \mathrm{H}), 2.25(\mathrm{~s}, 3 \mathrm{H}), 1.55\left(\mathrm{tq}, J^{1}=7.2 \mathrm{~Hz}, J^{2}=7.2 \mathrm{~Hz}, 2 \mathrm{H}\right), 0.96(\mathrm{t}, J$ $=7.4 \mathrm{~Hz}, 3 \mathrm{H}) ;{ }^{13} \mathbf{C ~ N M R}\left(75 \mathrm{MHz}, \mathrm{CDCl}_{3}, 300 \mathrm{~K}\right): \delta(\mathrm{ppm})=137.4(\mathrm{C}), 131.4(\mathrm{CH})$, 
$128.9(\mathrm{CH}), 121.0(\mathrm{C}), 89.4(\mathrm{C}), 80.7(\mathrm{C}), 22.3\left(\mathrm{CH}_{2}\right), 21.4\left(\mathrm{CH}_{2}\right), 21.3\left(\mathrm{CH}_{3}\right), 13.5$ $\left(\mathrm{CH}_{3}\right)$; EI-MS (m/z, relative intensity): $158\left(\mathrm{M}^{+}, 34\right), 143$ (38), 129 (100), 128 (63), 115 (25), 102 (7), 91 (4), 74 (10), 77 (11), 63 (6), 51 (4); IR (neat, $\mathrm{cm}^{-1}$ ): 3029w, 2963m, $2933 m, 2872 w, 2233 w, 1509 s, 1460 m, 1379 w, 1338 w, 1284 w, 1180 w, 1106 w, 1040 w$, $945 w, 816 s$.

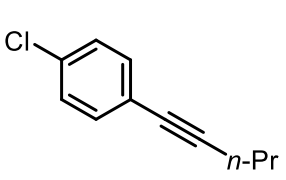

1-Chloro-4-(pent-1-yn-1-yl)benzene $(3 \mathrm{c})^{[3]}$ : The title compound was prepared according to general procedure (GP1) with

$\mathrm{Pd}\left(\mathrm{PPh}_{3}\right)_{2} \mathrm{Cl}_{2}(70.2 \mathrm{mg}, 0.100 \mathrm{mmol}, 1.0 \mathrm{~mol} \%), \mathrm{CuI}(38.1 \mathrm{mg}$, $0.200 \mathrm{mmol}, 2.0 \mathrm{~mol} \%)$, 1-chloro-4-iodobenzene (2.385 g, $10.00 \mathrm{mmol}, 1.0$ equiv), and pent-1-yne $\left(0.817 \mathrm{~g}, 12.0 \mathrm{mmol}, 1.2\right.$ equiv) in $\mathrm{Et}_{3} \mathrm{~N}(10 \mathrm{~mL})$ at room temperature for 12 hours. Purification via silica gel chromatography (Pentane) gave the desired product 3c as a light yellow oil in $86 \%$ yield (1.532 g). TLC $\mathbf{R}_{\mathrm{f}}=0.85$ (Pentane); ${ }^{1} \mathbf{H}$ NMR (300 $\left.\mathrm{MHz}, \mathrm{CDCl}_{3}, 300 \mathrm{~K}\right): \delta(\mathrm{ppm})=7.35(\mathrm{~d}, J=8.7 \mathrm{~Hz}, 2 \mathrm{H}), 7.28(\mathrm{~d}, J=8.9 \mathrm{~Hz}, 2 \mathrm{H}), 2.41$ (t, $J=7.0 \mathrm{~Hz}, 2 \mathrm{H}), 1.66\left(\mathrm{tq}, J^{1}=7.2 \mathrm{~Hz}, J^{2}=7.2 \mathrm{~Hz}, 2 \mathrm{H}\right), 1.07(\mathrm{t}, J=7.4 \mathrm{~Hz}, 3 \mathrm{H}) ;{ }^{13} \mathrm{C}$ NMR (75 MHz, $\left.\mathrm{CDCl}_{3}, 300 \mathrm{~K}\right): \delta(\mathrm{ppm})=133.4(\mathrm{C}), 132.8(\mathrm{CH}), 128.5(\mathrm{CH}), 122.6$ (C), $91.3(\mathrm{C}), 79.7(\mathrm{C}), 22.1\left(\mathrm{CH}_{2}\right), 21.4\left(\mathrm{CH}_{2}\right), 13.5\left(\mathrm{CH}_{3}\right)$.

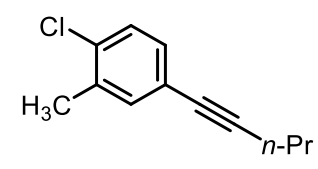

1-Chloro-2-methyl-4-(pent-1-yn-1-yl)benzene (3d): The title compound was prepared according to general procedure (GP1) with $\mathrm{Pd}\left(\mathrm{PPh}_{3}\right)_{2} \mathrm{Cl}_{2}(112.3 \mathrm{mg}, 160.0 \mu \mathrm{mol}, 2.0 \mathrm{~mol} \%), \mathrm{CuI}(61.0$ mg, $320.0 \mathrm{mmol}, 4.0 \mathrm{~mol} \%)$, 4-bromo-1-chloro-2-methylbenzene (1.631 g, $8.000 \mathrm{mmol}$, 1.0 equiv), and pent-1-yne (1.306 g, $19.20 \mathrm{mmol}, 2.4$ equiv) in $i-\operatorname{Pr}_{2} \mathrm{NH}(15 \mathrm{~mL})$ at 80 ${ }^{\circ} \mathrm{C}$ for 12 hours. Purification via silica gel chromatography (Pentane) gave the desired product $\mathbf{3 d}$ as a light yellow solid in $61 \%$ yield $(0.933 \mathrm{~g})$. TLC $\mathbf{R}_{\mathrm{f}}=0.85$ (Pentane); ${ }^{1} \mathbf{H}$ NMR (300 MHz, $\left.\mathrm{CDCl}_{3}, 300 \mathrm{~K}\right): \delta(\mathrm{ppm})=7.18(\mathrm{~s}, 1 \mathrm{H}), 7.16(\mathrm{~d}, J=8.2 \mathrm{~Hz}, 1 \mathrm{H}), 7.07$ $\left(\mathrm{dd}, J^{1}=8.2 \mathrm{~Hz}, J^{2}=1.8 \mathrm{~Hz}, 1 \mathrm{H}\right), 2.29(\mathrm{t}, J=7.0 \mathrm{~Hz}, 2 \mathrm{H}), 2.25(\mathrm{~s}, 3 \mathrm{H}), 1.55\left(\mathrm{tq}, J^{1}=\right.$ $\left.7.2 \mathrm{~Hz}, J^{2}=7.2 \mathrm{~Hz}, 2 \mathrm{H}\right), 0.97(\mathrm{t}, J=7.4 \mathrm{~Hz}, 3 \mathrm{H}) ;{ }^{13} \mathbf{C ~ N M R}\left(75 \mathrm{MHz}, \mathrm{CDCl}_{3}, 300 \mathrm{~K}\right)$ : $\delta(\mathrm{ppm})=\delta 135.9(\mathrm{C}), 133.9(\mathrm{CH}), 133.7(\mathrm{C}), 130.1(\mathrm{CH}), 128.9(\mathrm{CH}), 122.6(\mathrm{C}), 90.8$ (C), $79.9(\mathrm{C}), 22.2\left(\mathrm{CH}_{2}\right), 21.4\left(\mathrm{CH}_{2}\right), 19.8\left(\mathrm{CH}_{3}\right), 13.5\left(\mathrm{CH}_{3}\right) ; \mathbf{H R M S}(\mathrm{ESI}) \mathrm{m} / \mathrm{z}=$ 
298.97512 calcd. for $\mathrm{C}_{12} \mathrm{H}_{13} \mathrm{ClAg}[\mathrm{M}+\mathrm{Ag}]^{+}$, found: 298.97533; IR (neat, $\mathrm{cm}^{-1}$ ): $2963 w$, $2872 m, 2230 w, 1888 w, 1761 w, 1594 w, 1478 s, 1381 w, 1338 w, 1272 w, 1180 w, 1137 w$, $1051 s, 883 m, 819 s, 773 w, 705 w$.

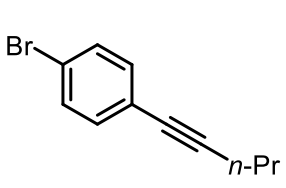

1-Bromo-4-(pent-1-yn-1-yl)benzene $(3 e)^{[4]}$ : The title compound was prepared according to general procedure (GP1) with $\mathrm{Pd}\left(\mathrm{PPh}_{3}\right)_{2} \mathrm{Cl}_{2}(70.2 \mathrm{mg}, 0.100 \mathrm{mmol}, 1.0 \mathrm{~mol} \%), \mathrm{CuI}(38.1 \mathrm{mg}$, $0.200 \mathrm{mmol}, 2.0 \mathrm{~mol} \%)$, 1-bromo-4-iodobenzene ( $8.490 \mathrm{~g}, 30.00 \mathrm{mmol}, 3.0$ equiv), and pent-1-yne (0.681 g, $10.0 \mathrm{mmol}, 1.0$ equiv) in $\mathrm{Et}_{3} \mathrm{~N}(10 \mathrm{~mL})$ at room temperature for 12 hours. Purification via silica gel chromatography (Pentane) gave the desired product 3e as a light yellow oil in $75 \%$ yield (1.670 g). TLC $\mathbf{R}_{\mathrm{f}}=0.85$ (Pentane); ${ }^{1} \mathbf{H}$ NMR (300 $\left.\mathrm{MHz}, \mathrm{CDCl}_{3}, 300 \mathrm{~K}\right): \delta(\mathrm{ppm})=7.33(\mathrm{~d}, J=8.5 \mathrm{~Hz}, 2 \mathrm{H}), 7.17(\mathrm{~d}, J=8.5 \mathrm{~Hz}, 2 \mathrm{H}), 2.29$ (t, $J=7.0 \mathrm{~Hz}, 2 \mathrm{H}), 1.55\left(\mathrm{tq}, J^{1}=7.2 \mathrm{~Hz}, J^{2}=7.5 \mathrm{~Hz}, 2 \mathrm{H}\right), 0.97(\mathrm{t}, J=7.4 \mathrm{~Hz}, 3 \mathrm{H}) ;{ }^{13} \mathrm{C}$ NMR (75 MHz, $\left.\mathrm{CDCl}_{3}, 300 \mathrm{~K}\right): \delta(\mathrm{ppm})=133.0(\mathrm{CH}), 131.4(\mathrm{CH}), 123.1(\mathrm{C}), 121.5$ (C), $91.6(\mathrm{C}), 79.7(\mathrm{C}), 22.1\left(\mathrm{CH}_{2}\right), 21.4\left(\mathrm{CH}_{2}\right), 13.5\left(\mathrm{CH}_{3}\right)$.

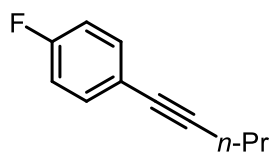

1-Fluoro-4-(pent-1-yn-1-yl)benzene $(3)^{[5]}$ : The title compound was prepared according to general procedure (GP1) with $\mathrm{Pd}\left(\mathrm{PPh}_{3}\right)_{2} \mathrm{Cl}_{2}(56.2 \mathrm{mg}, 80.0 \mu \mathrm{mol}, 1.0 \mathrm{~mol} \%), \mathrm{CuI}(30.5 \mathrm{mg}, 0.160$ mmol, $2.0 \mathrm{~mol} \%)$, 1-fluoro-4-iodobenzene (1.776 g, $8.000 \mathrm{mmol}, 1.0$ equiv), and pent1-yne $(0.654 \mathrm{~g}, 9.60 \mathrm{mmol}, 1.2$ equiv $)$ in $\mathrm{Et}_{3} \mathrm{~N}(8 \mathrm{~mL})$ at room temperature for 12 hours. Purification via silica gel chromatography (Pentane) gave the desired product $\mathbf{3 f}$ as a light yellow oil in 92\% yield (1.195 g). TLC $\mathbf{R}_{\mathrm{f}}=0.85$ (Pentane); ${ }^{1} \mathbf{H}$ NMR (300 MHz, $\left.\mathrm{CDCl}_{3}, 300 \mathrm{~K}\right): \delta(\mathrm{ppm})=7.32-7.27(\mathrm{~m}, 2 \mathrm{H}), 6.90(\mathrm{t}, J=8.6 \mathrm{~Hz}, 2 \mathrm{H}), 2.30(\mathrm{t}, J=7.0$ $\mathrm{Hz}, 2 \mathrm{H}), 1.55\left(\mathrm{tq}, J^{1}=7.2 \mathrm{~Hz}, J^{2}=7.2 \mathrm{~Hz}, 2 \mathrm{H}\right), 0.97(\mathrm{t}, J=7.4 \mathrm{~Hz}, 3 \mathrm{H}) ;{ }^{13} \mathbf{C}$ NMR $(75$ $\left.\mathrm{MHz}, \mathrm{CDCl}_{3}, 300 \mathrm{~K}\right): \delta(\mathrm{ppm})=162.0(\mathrm{~d}, J=248.0 \mathrm{~Hz}, \mathrm{CF}), 133.3(\mathrm{~d}, J=8.2 \mathrm{~Hz}, \mathrm{CH})$, $120.2(\mathrm{~d}, J=3.5 \mathrm{~Hz}, \mathrm{C}), 115.3(\mathrm{~d}, J=22.0 \mathrm{~Hz}, \mathrm{CH}), 89.9$ (C), $79.6(\mathrm{C}) .22 .2\left(\mathrm{CH}_{2}\right)$, $21.3\left(\mathrm{CH}_{2}\right), 13.5\left(\mathrm{CH}_{3}\right) ;{ }^{19} \mathbf{F}$ NMR $\left(282 \mathrm{MHz}, \mathrm{CDCl}_{3}, 300 \mathrm{~K}\right): \delta(\mathrm{ppm})=-112.5(\mathrm{~s}, 3 \mathrm{~F})$.

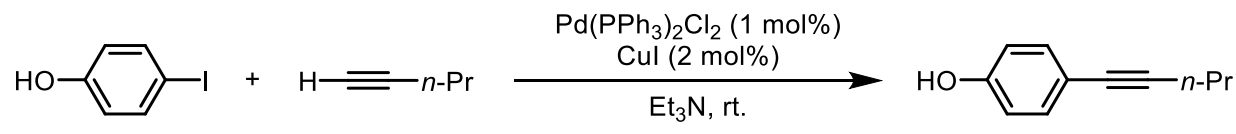



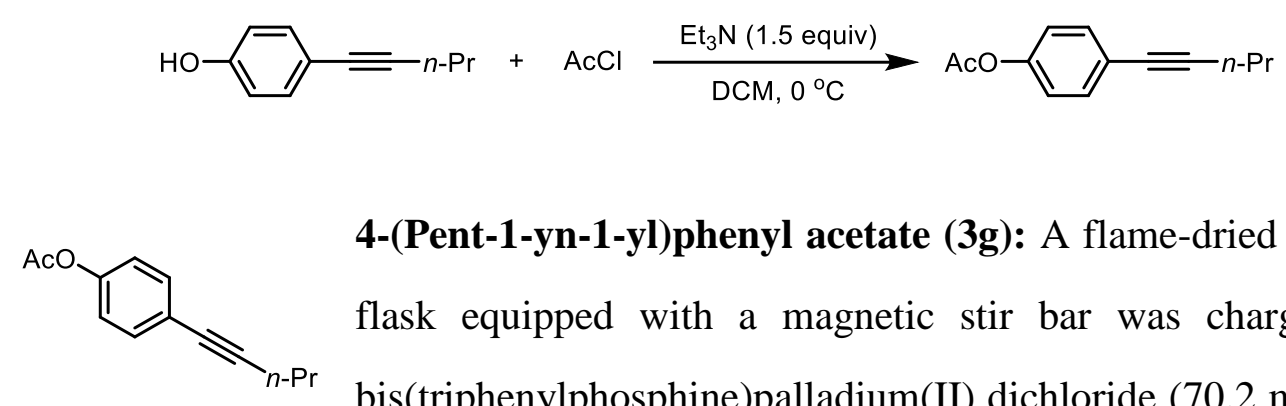

4-(Pent-1-yn-1-yl)phenyl acetate (3g): A flame-dried Schlenkflask equipped with a magnetic stir bar was charged with bis(triphenylphosphine)palladium(II) dichloride (70.2 mg, 0.100 mmol, $1.0 \mathrm{~mol} \%$ ) and copper(I) iodide (38.1 mg, $0.200 \mathrm{mmol}, 2.0 \mathrm{~mol} \%$ ), 4-iodophenol ( $2.200 \mathrm{~g}, 10.00 \mathrm{mmol}, 1.0$ equiv), sealed with a septum, and degassed by alternating vacuum evacuation and argon backfilling (three times) before $\mathrm{Et}_{3} \mathrm{~N}(10 \mathrm{~mL})$ was added. Pent-1-yne (0.820 g, $12.0 \mathrm{mmol}, 1.2$ equiv) was added to the resulting suspension subsequently. The reaction mixture was then stirred at room temperature for 12 hours. After the reaction was complete, the reaction mixture was diluted with $\mathrm{Et}_{2} \mathrm{O}(30 \mathrm{~mL})$ and filtrated through a pad of silica gel to remove most impurity. The solvent was removed under reduced pressure with the aid of a rotary evaporator and the crude residue was used in next step without further purification. Acetyl chloride $(0.640 \mathrm{~g}$, 8.13 mmol, 1.3 equiv) was slowly added to a solution of the crude 4-(pent-1-yn-1yl)phenol (1.000 g, $6.250 \mathrm{mmol}, 1.0$ equiv), triethylamine (0.950 g, $9.38 \mathrm{mmol}, 1.5$ equiv) and in $\mathrm{DCM}(10 \mathrm{~mL})$ at $0{ }^{\circ} \mathrm{C}$. The reaction mixture was stirred at room temperature for $6 \mathrm{~h}$, before being diluted with hexanes $(30 \mathrm{~mL})$. The solid precipitates were filtered off and the filtrate obtained was concentrated under reduced pressure with the aid of a rotary evaporator. The crude residue was purified through silica gel flash column chromatography (Pentane:EtOAc $=12: 1$ ) to give pure $\mathbf{3 g}$ as light yellow oil in $65 \%$ yield $(0.818 \mathrm{~g})$. TLC Rf $=0.65$ (Pentane:EtOAc $=4: 1) ;{ }^{1} \mathbf{H}$ NMR $(300 \mathrm{MHz}$, $\left.\mathrm{CDCl}_{3}, 300 \mathrm{~K}\right): \delta(\mathrm{ppm})=7.32(\mathrm{~d}, J=8.6 \mathrm{~Hz}, 2 \mathrm{H}), 6.93(\mathrm{~d}, J=8.6 \mathrm{~Hz}, 2 \mathrm{H}), 2.29(\mathrm{t}, J$ $=7.0 \mathrm{~Hz}, 2 \mathrm{H}), 2.20(\mathrm{~s}, 3 \mathrm{H}), 1.55\left(\mathrm{tq}, J^{1}=7.2 \mathrm{~Hz}, J^{2}=7.2 \mathrm{~Hz}, 2 \mathrm{H}\right), 0.96(\mathrm{t}, J=7.4 \mathrm{~Hz}$, $3 \mathrm{H}) ;{ }^{13} \mathrm{C} \mathrm{NMR}\left(75 \mathrm{MHz}, \mathrm{CDCl}_{3}, 300 \mathrm{~K}\right): \delta(\mathrm{ppm})=169.1(\mathrm{C}), 149.8(\mathrm{C}), 132.6(\mathrm{CH})$, $121.8(\mathrm{C}), 121.4(\mathrm{CH}), 90.3(\mathrm{C}), 79.9(\mathrm{C}), 22.1\left(\mathrm{CH}_{2}\right), 21.3\left(\mathrm{CH}_{2}\right), 21.1\left(\mathrm{CH}_{3}\right), 13.5$ $\left(\mathrm{CH}_{3}\right)$; HRMS (ESI) $m / z=225.0886$ calcd. for $\mathrm{C}_{13} \mathrm{H}_{14} \mathrm{O}_{2} \mathrm{Na}[\mathrm{M}+\mathrm{Na}]^{+}$, found: 225.0889; IR (neat, $\mathrm{cm}^{-1}$ ): 2964w, 2396w, 2238w, 1765s, 1601w, 1504s, 1369m, 1189s, 1098w, $1014 m, 909 s, 847 s, 726 w, 690 w$. 


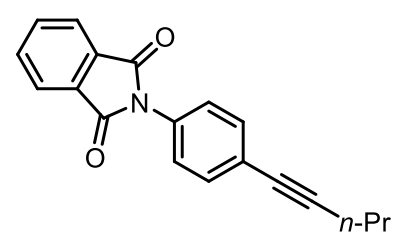

\section{2-(4-(Pent-1-yn-1-yl)phenyl)isoindoline-1,3-dione (3h):}

The title compound was prepared according to general procedure (GP1) with $\mathrm{Pd}\left(\mathrm{PPh}_{3}\right)_{2} \mathrm{Cl}_{2}(112.4 \mathrm{mg}, 0.1600$ mmol, $2.0 \mathrm{~mol} \%)$, CuI (61.0 mg, $0.320 \mathrm{mmol}, 4.0 \mathrm{~mol} \%)$, 2-(4-bromophenyl)isoindoline-1,3-dione ( $2.41 \mathrm{~g}, 8.0 \mathrm{mmol}, 1.0$ equiv), and pent-1-yne (1.308 g, $19.20 \mathrm{mmol}, 2.4$ equiv) in $i-\mathrm{Pr}_{2} \mathrm{NH}(16 \mathrm{~mL})$ and $\mathrm{THF}(16 \mathrm{~mL})$ at $80{ }^{\circ} \mathrm{C}$ for 24 hours. Purification via silica gel chromatography (Pentane:EtOAc $=16: 1)$ gave the desired product $3 \mathbf{h}$ as a gray solid in $58 \%$ yield $(1.360 \mathrm{~g})$. TLC $\mathbf{R}_{\mathrm{f}}=0.55$ (Pentane:EtOAc = 4:1); MP: $119^{\circ} \mathrm{C}$, (decomp.); ${ }^{1} \mathbf{H}$ NMR $\left(300 \mathrm{MHz}, \mathrm{CDCl}_{3}, 300 \mathrm{~K}\right)$ : $\delta(\mathrm{ppm})=7.95\left(\mathrm{dd}, J^{1}=5.5 \mathrm{~Hz}, J^{2}=3.0 \mathrm{~Hz}, 2 \mathrm{H}\right), 7.78\left(\mathrm{dd}, J^{1}=5.5 \mathrm{~Hz}, J^{2}=3.1 \mathrm{~Hz}\right.$, 2H), $7.51(\mathrm{~d}, J=8.6 \mathrm{~Hz}, 2 \mathrm{H}), 7.39(\mathrm{~d}, J=8.7 \mathrm{~Hz}, 2 \mathrm{H}), 2.40(\mathrm{t}, J=7.0 \mathrm{~Hz}, 2 \mathrm{H}), 1.64$ $\left(\mathrm{tq}, J^{1}=7.2 \mathrm{~Hz}, J^{2}=7.2 \mathrm{~Hz}, 2 \mathrm{H}\right), 1.06(\mathrm{t}, J=7.4 \mathrm{~Hz}, 3 \mathrm{H}) ;{ }^{13} \mathbf{C ~ N M R}\left(75 \mathrm{MHz}, \mathrm{CDCl}_{3}\right.$, $300 \mathrm{~K}): \delta(\mathrm{ppm})=\delta 167.0(\mathrm{C}), 134.4(\mathrm{CH}), 132.2(\mathrm{CH}), 131.7(\mathrm{C}), 130.8(\mathrm{C}), 126.1$ $(\mathrm{CH}), 124.0(\mathrm{C}), 123.7(\mathrm{CH}), 91.4(\mathrm{C}), 80.1(\mathrm{C}), 22.1\left(\mathrm{CH}_{2}\right), 21.4\left(\mathrm{CH}_{2}\right), 13.5\left(\mathrm{CH}_{3}\right)$; HRMS (ESI) $m / z=312.0995$ calcd. for $\mathrm{C}_{19} \mathrm{H}_{15} \mathrm{NO}_{2} \mathrm{Na}[\mathrm{M}+\mathrm{Na}]^{+}$, found: $312.1001 ;$ IR (neat, $\mathrm{cm}^{-1}$ ): 3052w, 2966w, 2240w, 1709s, 1610w, 1513s, 1465w, 1382s, 1285w, 1222s, $1177 w, 1116 m, 1080 m, 948 w, 883 m, 834 m, 790 w, 714 s, 667 w$.

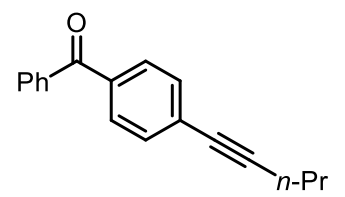

(4-(Pent-1-yn-1-yl)phenyl)(phenyl)methanone (3i): The title compound was prepared according to general procedure (GP1) with $\mathrm{Pd}\left(\mathrm{PPh}_{3}\right)_{2} \mathrm{Cl}_{2}(56.2 \mathrm{mg}, 80.0 \mu \mathrm{mol}, 1.0 \mathrm{~mol} \%), \mathrm{CuI}(30.5$ $\mathrm{mg}, 0.160 \mathrm{mmol}, 2.0 \mathrm{~mol} \%$ ), (4-bromophenyl)(phenyl)methanone (2.09 g, $8.00 \mathrm{mmol}$, 1.0 equiv), and pent-1-yne ( $0.654 \mathrm{~g}, 9.60 \mathrm{mmol}, 1.2$ equiv) in $i-\mathrm{Pr}_{2} \mathrm{NH}(10 \mathrm{~mL})$ and at $80^{\circ} \mathrm{C}$ for 24 hours. Purification via silica gel chromatography (Pentane:EtOAc $=200: 1$, then 50:1) gave the desired product $3 \mathbf{i}$ as a yellow solid in $92 \%$ yield (1.836 $\mathrm{g}$ ). TLC $\mathbf{R}_{\mathrm{f}}=0.55$ (Pentane:EtOAc $\left.=20: 1\right) ; \mathbf{M P}: 28{ }^{\circ} \mathrm{C} ;{ }^{1} \mathbf{H}$ NMR $\left(300 \mathrm{MHz}, \mathrm{CDCl}_{3}, 300 \mathrm{~K}\right): \delta$ $(\mathrm{ppm})=7.78-7.72(\mathrm{~m}, 4 \mathrm{H}), 7.61-7.55(\mathrm{~m}, 1 \mathrm{H}), 7.50-7.45(\mathrm{~m}, 4 \mathrm{H}), 2.42(\mathrm{t}, J=7.0$ $\mathrm{Hz}, 2 \mathrm{H}), 1.66$ (tq, $\left.J^{1}=7.2 \mathrm{~Hz}, J^{2}=7.2 \mathrm{~Hz}, 2 \mathrm{H}\right), 1.06$ (t, $\left.J=7.4 \mathrm{~Hz}, 3 \mathrm{H}\right) ;{ }^{13} \mathbf{C} \mathbf{~ N M R}(75$ $\left.\mathrm{MHz}, \mathrm{CDCl}_{3}, 300 \mathrm{~K}\right): \delta(\mathrm{ppm})=196.0(\mathrm{C}), 137.6(\mathrm{C}), 136.1(\mathrm{C}), 132.4(\mathrm{CH}), 131.4$ $(\mathrm{CH}), 130.0(\mathrm{CH}), 129.9(\mathrm{CH}), 128.6(\mathrm{CH}), 128.3(\mathrm{C}), 93.9(\mathrm{C}), 80.3(\mathrm{C}), 22.0\left(\mathrm{CH}_{2}\right)$, 
$21.5\left(\mathrm{CH}_{2}\right), 13.5\left(\mathrm{CH}_{3}\right)$; HRMS (ESI) $m / z=271.1093$ calcd. for $\mathrm{C}_{18} \mathrm{H}_{16} \mathrm{ONa}[\mathrm{M}+\mathrm{Na}]^{+}$, found: 271.1094; IR (neat, $\mathrm{cm}^{-1}$ ): 3058w, 2964w, 2361w, 2237w, 1659s, 1600s, 1447w, $1404 w, 1276 s, 1176 w, 937 w, 852 w, 792 w, 741 w, 700 m, 665 w$.

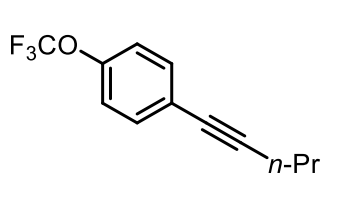

1-(Pent-1-yn-1-yl)-4-(trifluoromethoxy)benzene $\quad(3 \mathbf{3 j}):$ The title compound was prepared according to general procedure

(GP1) with $\mathrm{Pd}\left(\mathrm{PPh}_{3}\right)_{2} \mathrm{Cl}_{2}(56.2 \mathrm{mg}, 80.0 \mu \mathrm{mol}, 1.0 \mathrm{~mol} \%), \mathrm{CuI}$ (30.5 mg, $0.160 \mathrm{mmol}, 2.0 \mathrm{~mol} \%$ ), 1-iodo-4-(trifluoromethoxy)benzene (2.304 g, 8.000 mmol, 1.0 equiv), and pent-1-yne ( $0.654 \mathrm{~g}, 9.60 \mathrm{mmol}, 1.2$ equiv) in $\mathrm{Et}_{3} \mathrm{~N}$ (8 mL) at room temperature for 12 hours. Purification via silica gel chromatography (Pentane:EtOAc $=200: 1$ ) gave the desired product $\mathbf{3 j}$ as a light yellow oil in $81 \%$ yield $\left(1.469\right.$ g). TLC $\mathbf{R}_{\mathrm{f}}=0.8$ (Pentane:EtOAc $\left.=20: 1\right) ;{ }^{1} \mathbf{H}$ NMR $\left(300 \mathrm{MHz}, \mathrm{CDCl}_{3}, 300 \mathrm{~K}\right)$ : $\delta(\mathrm{ppm})=7.41(\mathrm{~d}, J=8.7 \mathrm{~Hz}, 2 \mathrm{H}), 7.13(\mathrm{~d}, J=8.7 \mathrm{~Hz}, 2 \mathrm{H}), 2.38(\mathrm{t}, J=7.0 \mathrm{~Hz}, 2 \mathrm{H})$, $1.63\left(\mathrm{tq}, J^{1}=7.2 \mathrm{~Hz}, J^{2}=7.2 \mathrm{~Hz}, 2 \mathrm{H}\right), 1.05(\mathrm{t}, J=7.4 \mathrm{~Hz}, 3 \mathrm{H}) ;{ }^{13} \mathrm{C}$ NMR $(75 \mathrm{MHz}$, $\left.\mathrm{CDCl}_{3}, 300 \mathrm{~K}\right): \delta(\mathrm{ppm})=148.4(\mathrm{C}), 133.0(\mathrm{CH}), 123.0(\mathrm{C}), 120.6(\mathrm{CH}), 120.4(\mathrm{q}, J=$ $\left.257.4 \mathrm{~Hz}, \mathrm{CF}_{3}\right), 91.3(\mathrm{C}), 79.4(\mathrm{C}), 22.1\left(\mathrm{CH}_{2}\right), 21.3\left(\mathrm{CH}_{2}\right), 13.5\left(\mathrm{CH}_{3}\right) ;{ }^{19} \mathbf{F}$ NMR $(282$ $\left.\mathrm{MHz}, \mathrm{CDCl}_{3}, 300 \mathrm{~K}\right): \delta(\mathrm{ppm})=-57.9(\mathrm{~s}, 3 \mathrm{~F})$; EI-MS (m/z, relative intensity) 228 $\left(\mathrm{M}^{+}, 72\right), 199$ (70), 143 (73), 133 (31), 128 (68), 127 (100), 115 (55), 102 (77), 69 (35); IR (neat, $\mathrm{cm}^{-1}$ ): 2967w, 2232w, 1507s, 1463w, 1255s, 1220s, 1166s, 1019w, 922w, $850 m, 666 w$.

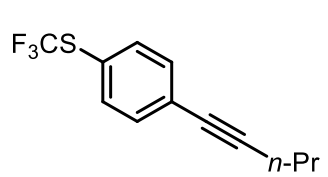

(4-(Pent-1-yn-1-yl)phenyl)(trifluoromethyl)sulfane (3k): The title compound was prepared according to general procedure (GP1) with $\mathrm{Pd}\left(\mathrm{PPh}_{3}\right)_{2} \mathrm{Cl}_{2}(56.2 \mathrm{mg}, 80.0 \mu \mathrm{mol}, 1.0 \mathrm{~mol} \%), \mathrm{CuI}$ (30.5 mg, $0.160 \mathrm{mmol}, 2.0 \mathrm{~mol} \%$ ), (4-bromophenyl)(trifluoromethyl)sulfane (2.057 g, $8.0 \mathrm{mmol}, 1.0$ equiv), and pent- $1-\mathrm{yne}\left(0.654 \mathrm{~g}, 9.60 \mathrm{mmol}, 1.2\right.$ equiv) in $i$ - $\operatorname{Pr}_{2} \mathrm{NH}$ (10 $\mathrm{mL}$ ) at $80{ }^{\circ} \mathrm{C}$ for 12 hours. Purification via silica gel chromatography (Pentane) gave the desired product 3k as a light yellow oil in $61 \%$ yield $(1.191 \mathrm{~g})$. TLC $\mathbf{R}_{\mathrm{f}}=0.7$ (Pentane); ${ }^{1} \mathbf{H}$ NMR $\left(300 \mathrm{MHz}, \mathrm{CDCl}_{3}, 300 \mathrm{~K}\right): \delta(\mathrm{ppm})=7.48(\mathrm{~d}, J=8.2 \mathrm{~Hz}, 2 \mathrm{H})$, $7.34(\mathrm{~d}, J=8.4 \mathrm{~Hz}, 2 \mathrm{H}), 2.32(\mathrm{t}, J=7.0 \mathrm{~Hz}, 2 \mathrm{H}), 1.56\left(\mathrm{tq}, J^{1}=7.2 \mathrm{~Hz}, J^{2}=7.2 \mathrm{~Hz}, 2 \mathrm{H}\right)$, 
$0.97(\mathrm{t}, J=7.4 \mathrm{~Hz}, 3 \mathrm{H}) ;{ }^{13} \mathbf{C} \mathbf{N M R}\left(75 \mathrm{MHz}, \mathrm{CDCl}_{3}, 300 \mathrm{~K}\right): \delta(\mathrm{ppm})=136.0(\mathrm{CH})$, $132.4(\mathrm{CH}), 129.5$ (q, $\left.J=308.2 \mathrm{~Hz}, \mathrm{CF}_{3}\right), 127.1(\mathrm{C}), 123.3$ (q, $\left.J=2.0 \mathrm{~Hz}, \mathrm{C}\right), 93.5(\mathrm{C})$, $79.7(\mathrm{C}), 22.0\left(\mathrm{CH}_{2}\right), 21.4\left(\mathrm{CH}_{2}\right), 13.5\left(\mathrm{CH}_{3}\right) ;{ }^{19} \mathbf{F} \mathbf{N M R}\left(282 \mathrm{MHz}, \mathrm{CDCl}_{3}, 300 \mathrm{~K}\right): \delta$ $(\mathrm{ppm})=-42.7(\mathrm{~s}, 3 \mathrm{~F})$; EI-MS $\left(\mathrm{m} / \mathrm{z}\right.$, relative intensity): $244\left(\mathbf{M}^{+}, 100\right), 215(74), 146$ (47), 128 (61), 115 (18), 102 (17), 89 (9), 69 (11), 45 (4), 39 (5); IR (neat, cm ${ }^{-1}$ ): 2967w, $2875 w, 2238 w, 1592 w, 1491 w, 1397 w, 1339 w, 1283 w, 1115 s, 1016 w, 833 m, 756 w$.

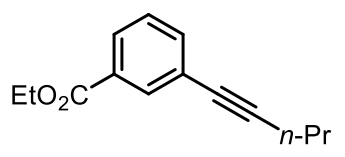

Ethyl 3-(pent-1-yn-1-yl)benzoate (31): The title compound was prepared according to general procedure (GP1) with

$\mathrm{Pd}\left(\mathrm{PPh}_{3}\right)_{2} \mathrm{Cl}_{2}(56.2 \mathrm{mg}, 80.0 \mu \mathrm{mol}, 1.0 \mathrm{~mol} \%), \mathrm{CuI}(30.5 \mathrm{mg}$, $0.160 \mathrm{mmol}, 2.0 \mathrm{~mol} \%)$, ethyl 3-iodobenzoate (2.209 g, $8.000 \mathrm{mmol}, 1.0$ equiv), and pent-1-yne (0.654 g, $9.60 \mathrm{mmol}, 1.2$ equiv) in $\mathrm{Et}_{3} \mathrm{~N}(8 \mathrm{~mL})$ at room temperature for 12 hours. Purification via silica gel chromatography (Pentane:EtOAc $=100: 1)$ gave the desired product $3 \mathrm{I}$ as a light yellow oil in 95\% yield (1.648 g). TLC $\mathbf{R}_{\mathrm{f}}=0.5$ (Pentane:EtOAc = 20:1); ${ }^{1} \mathbf{H}$ NMR $\left(300 \mathrm{MHz}, \mathrm{CDCl}_{3}, 300 \mathrm{~K}\right): \delta(\mathrm{ppm})=7.99(\mathrm{~s}, 1 \mathrm{H})$, $7.86\left(\mathrm{dd}, J^{1}=7.8 \mathrm{~Hz}, J^{2}=1.1 \mathrm{~Hz}, 1 \mathrm{H}\right), 7.48\left(\mathrm{dd}, J^{1}=7.7 \mathrm{~Hz}, J^{2}=1.0 \mathrm{~Hz}, 1 \mathrm{H}\right), 7.27(\mathrm{t}$, $J=7.8 \mathrm{~Hz}, 1 \mathrm{H}), 4.30(\mathrm{q}, J=7.1 \mathrm{~Hz}, 2 \mathrm{H}), 2.32(\mathrm{t}, J=7.0 \mathrm{~Hz}, 2 \mathrm{H}), 1.57\left(\mathrm{tq}, J^{1}=7.2 \mathrm{~Hz}\right.$, $\left.J^{2}=6.9 \mathrm{~Hz}, 2 \mathrm{H}\right), 1.31(\mathrm{t}, J=7.1 \mathrm{~Hz}, 3 \mathrm{H}), 0.98(\mathrm{t}, J=7.3 \mathrm{~Hz}, 3 \mathrm{H}) ;{ }^{13} \mathbf{C} \mathbf{N M R}(75 \mathrm{MHz}$, $\left.\mathrm{CDCl}_{3}, 300 \mathrm{~K}\right): \delta(\mathrm{ppm})=166.0(\mathrm{C}), 135.6(\mathrm{CH}), 132.6(\mathrm{CH}), 130.6(\mathrm{C}), 128.5(\mathrm{CH})$, $128.2(\mathrm{CH}), 124.5(\mathrm{C}), 91.3(\mathrm{C}), 79.9(\mathrm{C}), 61.1\left(\mathrm{CH}_{2}\right), 22.1\left(\mathrm{CH}_{2}\right), 21.3\left(\mathrm{CH}_{2}\right), 14.3$ $\left(\mathrm{CH}_{3}\right), 13.5\left(\mathrm{CH}_{3}\right)$; HRMS (ESI) $m / z=239.1043$ calcd. for $\mathrm{C}_{14} \mathrm{H}_{16} \mathrm{O}_{2} \mathrm{Na}[\mathrm{M}+\mathrm{Na}]^{+}$, found: 239.1049; IR (neat, $\mathrm{cm}^{-1}$ ): 2965w, 2234w, 2065w, 1721s, 1602w, 1464w, 1368w, $1292 s, 1224 s, 1169 w, 1105 s, 1024 m, 913 w, 817 w, 754 s, 685 w$.

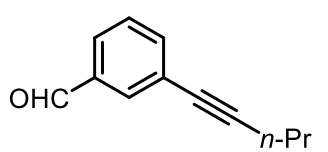

3-(Pent-1-yn-1-yl)benzaldehyde (3m): The title compound was prepared according to general procedure (GP1) with $\mathrm{Pd}\left(\mathrm{PPh}_{3}\right)_{2} \mathrm{Cl}_{2}$ (112.3 mg, $160.0 \mu \mathrm{mol}, 2.0 \mathrm{~mol} \%)$, CuI (61.0 mg, $320.0 \mathrm{mmol}$, $4.0 \mathrm{~mol} \%)$, 3-bromobenzaldehyde (1.48 g, $8.00 \mathrm{mmol}, 1.0$ equiv), and pent-1-yne (1.308 g, $19.20 \mathrm{mmol}, 2.4$ equiv) in $i-\mathrm{Pr}_{2} \mathrm{NH}(8 \mathrm{~mL})$ and at $80{ }^{\circ} \mathrm{C}$ for 24 hours. Purification via silica gel chromatography (Pentane:EtOAc $=200: 1)$ gave the desired 
product $\mathbf{3 m}$ as a yellow oil in $69 \%$ yield $(0.953 \mathrm{~g})$. TLC $\mathbf{R}_{\mathrm{f}}=0.6$ (Pentane:EtOAc $=$ 20:1); ${ }^{1} \mathbf{H}$ NMR (300 MHz, $\left.\mathrm{CDCl}_{3}, 300 \mathrm{~K}\right): \delta(\mathrm{ppm})=9.90$ (s, 1H), 7.81 (s, 1H), 7.69 $\left(\mathrm{dt}, J^{1}=7.7 \mathrm{~Hz}, J^{2}=1.4 \mathrm{~Hz}, 1 \mathrm{H}\right), 7.55\left(\mathrm{dt}, J^{1}=7.7 \mathrm{~Hz}, J^{2}=1.4 \mathrm{~Hz}, 1 \mathrm{H}\right), 7.37(\mathrm{t}, J=$ $7.7 \mathrm{~Hz}, 1 \mathrm{H}), 2.33$ (t, $J=7.0 \mathrm{~Hz}, 2 \mathrm{H}), 1.57$ (tq, $\left.J^{1}=7.2 \mathrm{~Hz}, J^{2}=7.5 \mathrm{~Hz}, 2 \mathrm{H}\right), 0.98$ (t, $J$ $=7.4 \mathrm{~Hz}, 3 \mathrm{H}) ;{ }^{13} \mathbf{C ~ N M R}\left(75 \mathrm{MHz}, \mathrm{CDCl}_{3}, 300 \mathrm{~K}\right): \delta(\mathrm{ppm})=191.7(\mathrm{CH}), 137.1(\mathrm{CH})$, $136.4(\mathrm{C}), 132.9(\mathrm{CH}), 128.9(\mathrm{C}), 128.2(\mathrm{CH}), 125.3(\mathrm{C}), 92.1(\mathrm{C}), 79.4(\mathrm{C}), 22.0\left(\mathrm{CH}_{2}\right)$, $21.3\left(\mathrm{CH}_{2}\right), 13.5\left(\mathrm{CH}_{3}\right)$; HRMS (ESI) $m / z=195.0780$ calcd. for $\mathrm{C}_{12} \mathrm{H}_{12} \mathrm{ONa}[\mathrm{M}+\mathrm{Na}]^{+}$, found: 195.0783; IR (neat, $\mathrm{cm}^{-1}$ ): 2965w, 2873w, 2725w, 2232w, 1797w, 1700s, 1600w, $1434 w, 1382 w, 1284 w, 1159 w, 1031 w, 1031 w, 1031 w, 1031 w, 1031 w, 1031 w, 910,796$ $757,684,649$.

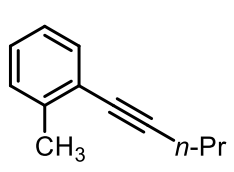

1-Methyl-2-(pent-1-yn-1-yl)benzene (3n): The title compound was prepared according to general procedure $\left(\right.$ GP1) with $\mathrm{Pd}\left(\mathrm{PPh}_{3}\right)_{2} \mathrm{Cl}_{2}$ (56.2 mg, $80.0 \mu \mathrm{mol}, 1.0 \mathrm{~mol} \%$ ), CuI (30.5 mg, $0.160 \mathrm{mmol}, 2.0$ mol\%), 1-iodo-2-methylbenzene (1.744 g, $8.000 \mathrm{mmol}, 1.0$ equiv), and pent-1-yne (0.654 g, $9.60 \mathrm{mmol}, 1.2$ equiv) in $\mathrm{Et}_{3} \mathrm{~N}(8 \mathrm{~mL})$ at room temperature for 12 hours. Purification via silica gel chromatography (Pentane) gave the desired product $\mathbf{3 n}$ as a light yellow oil in $81 \%$ yield (1.023 g). TLC $\mathbf{R}_{\mathrm{f}}=0.8$ (Pentane); ${ }^{1} \mathbf{H}$ NMR $(300 \mathrm{MHz}$, $\left.\mathrm{CDCl}_{3}, 300 \mathrm{~K}\right): \delta(\mathrm{ppm})=7.42(\mathrm{~d}, J=7.2 \mathrm{~Hz}, 1 \mathrm{H}), 7.22-7.20(\mathrm{~m}, 2 \mathrm{H}), 7.19-7.12$ (m, 1H), 2.48 (t, $J=6.9 \mathrm{~Hz}, 2 \mathrm{H}), 2.47(\mathrm{~s}, 3 \mathrm{H}), 1.70\left(\mathrm{tq}, J^{1}=7.2 \mathrm{~Hz}, J^{2}=7.2 \mathrm{~Hz}, 2 \mathrm{H}\right)$, $1.12(\mathrm{t}, J=7.4 \mathrm{~Hz}, 3 \mathrm{H}) ;{ }^{13} \mathrm{C}$ NMR $\left(75 \mathrm{MHz}, \mathrm{CDCl}_{3}, 300 \mathrm{~K}\right): \delta(\mathrm{ppm})=139.9(\mathrm{C})$, $131.8(\mathrm{CH}), 129.2(\mathrm{CH}), 127.4(\mathrm{CH}), 125.4(\mathrm{CH}), 123.9(\mathrm{C}), 94.2(\mathrm{C}), 79.6(\mathrm{C}), 22.4$ $\left(\mathrm{CH}_{2}\right), 21.5\left(\mathrm{CH}_{2}\right), 20.7\left(\mathrm{CH}_{3}\right), 13.5\left(\mathrm{CH}_{3}\right)$; EI-MS $\left(\mathrm{m} / \mathrm{z}\right.$, relative intensity): $158\left(\mathrm{M}^{+}\right.$, 29), 143 (25), 129 (86), 128 (100), 127 (33), 115 (31), 102 (10), 89 (4), 77 (10), 63 (5), 51 (4); IR (neat, $\mathrm{cm}^{-1}$ ): 3023w, 2964w, 2872w, 2233w, 1601w, 1457w, 1379s, 1338w, $1279 w, 1115 w, 1041 w, 942 w, 879 s, 756 s$.

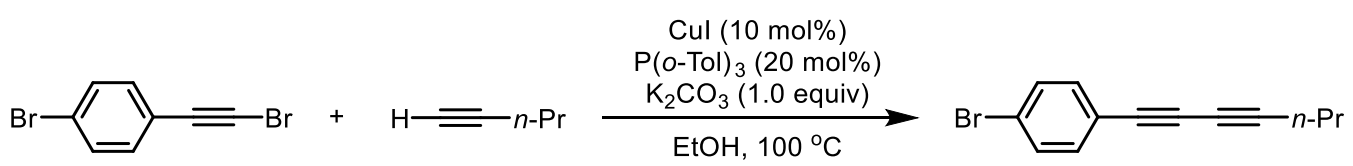




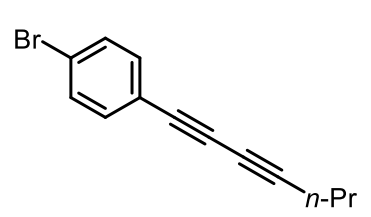

1-Bromo-4-(hepta-1, 3-diyn-1-yl)benzene (3o $)^{[6]}$ : A flamedried Schlenk-flask equipped with a magnetic stir bar was charged with copper(I) iodide (95.2 mg, $0.500 \mathrm{mmol}, 10 \mathrm{~mol} \%)$, tri(o-tolyl)phosphine $(30.4 \mathrm{mg}, 1.00 \mathrm{mmol}, 20 \mathrm{~mol} \%)$, potassium carbonate $(0.691 \mathrm{~g}$, $5.00 \mathrm{mmol}, 1.0$ equiv), and 1-bromo-4-(bromoethynyl)benzene ${ }^{[7]}(1.290 \mathrm{~g}, 5.000 \mathrm{mmol}$, 1.0 equiv), sealed with a septum, and degassed by alternating vacuum evacuation and argon backfilling (three times) before anhydrous ethanol $(20 \mathrm{~mL})$ was added. Pent-1yne (0.409 g, $6.00 \mathrm{mmol}, 1.2$ equiv) was added to the resulting suspension subsequently. The reaction mixture was then stirred at $100{ }^{\circ} \mathrm{C}$ for 24 hours. After the reaction was complete, the reaction mixture was diluted with EtOAc $(50 \mathrm{~mL})$ and filtrated through filtered through a pad of Celite. The solvent was removed under reduced pressure with the aid of a rotary evaporator and the crude residue was purified by a silica gel column chromatography (Pentane) to give pure 1,3-diyne 3o as a white solid in 70\% yield (0.858 g). TLC $\mathbf{R}_{\mathrm{f}}=0.8$ (Pentane); MP: $85^{\circ} \mathrm{C} ;{ }^{1} \mathbf{H}$ NMR $\left(300 \mathrm{MHz}, \mathrm{CDCl}_{3}, 300 \mathrm{~K}\right): \delta$ $(\mathrm{ppm})=7.35(\mathrm{~d}, J=8.6 \mathrm{~Hz}, 2 \mathrm{H}), 7.24(\mathrm{~d}, J=8.6 \mathrm{~Hz}, 2 \mathrm{H}), 2.26(\mathrm{t}, J=7.0 \mathrm{~Hz}, 2 \mathrm{H}), 1.53$ $\left(\mathrm{tq}, J^{1}=7.2 \mathrm{~Hz}, J^{2}=7.5 \mathrm{~Hz}, 2 \mathrm{H}\right), 0.95(\mathrm{t}, J=7.4 \mathrm{~Hz}, 3 \mathrm{H}) ;{ }^{13} \mathbf{C ~ N M R}\left(75 \mathrm{MHz}, \mathrm{CDCl}_{3}\right.$, $300 \mathrm{~K}): \delta(\mathrm{ppm})=133.8(\mathrm{CH}), 131.6(\mathrm{CH}), 123.1(\mathrm{C}), 121.1(\mathrm{C}), 85.4(\mathrm{C}), 75.6(\mathrm{C})$, $73.5(\mathrm{C}), 65.1(\mathrm{C}), 21.7\left(\mathrm{CH}_{2}\right), 21.6\left(\mathrm{CH}_{2}\right), 13.5\left(\mathrm{CH}_{3}\right) ;$ HRMS $(\mathrm{ESI}) \mathrm{m} / \mathrm{z}=254.90691$ calcd. for $\mathrm{C}_{13} \mathrm{H}_{11} \mathrm{BrAg}[\mathrm{M}+\mathrm{Ag}]^{+}$, found: 354.90750; IR (neat, $\mathrm{cm}^{-1}$ ): 3086w, 2959m, $2867 w, 2243 w, 2156 w, 1904 w, 1647 w, 1582 w, 1473 m, 1390 m, 1346 w, 1267 m, 1096 w$, 1065m, 1007m, 907m, 820s, 732s, 650w.

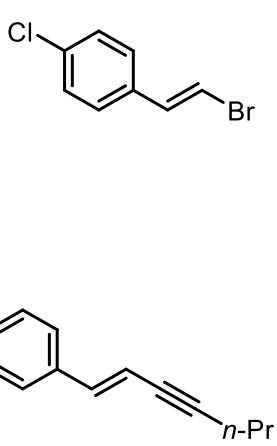

(E)-1-Chloro-4-(hept-1-en-3-yn-1-yl)benzene $\quad(3 p)^{[8]}: \quad$ A flame-dried Schlenk-flask equipped with a magnetic stir bar was charged with tetrakis(triphenylphosphine)palladium(0) (57.7 mg, $50.0 \mu \mathrm{mol}, 1.0 \mathrm{~mol} \%$ ), copper(I) iodide (19.0 mg, $0.100 \mathrm{mmol}, 2.0 \mathrm{~mol} \%$ ), 
and (E)-1-(2-bromovinyl)-4-chlorobenzene ${ }^{[9]}(1.080 \mathrm{~g}, 5.000 \mathrm{mmol}, 1.0$ equiv), sealed with a septum, and degassed by alternating vacuum evacuation and argon backfilling (three times) before diethylamine $(12 \mathrm{~mL})$ was added. Pent-1-yne $(0.409 \mathrm{~g}, 0.600 \mathrm{mmol}$, 1.2 equiv) was added to the resulting suspension subsequently. The reaction mixture was then stirred at room temperature for 12 hours. After the reaction was complete, the reaction mixture was diluted with $\mathrm{Et}_{2} \mathrm{O}(30 \mathrm{~mL})$ and filtrated through a small pad of silica gel. The solvent was removed under reduced pressure with the aid of a rotary evaporator and the crude residue was purified by a silica gel column chromatography (Pentane) to give pure 1,3 enyne $\mathbf{3 p}$ as a white solid in $56 \%$ yield $(0.570 \mathrm{~g})$. TLC $\mathbf{R}_{\mathrm{f}}=$ 0.7 (Pentane); MP: $32{ }^{\circ} \mathrm{C} ;{ }^{1} \mathbf{H}$ NMR $\left(300 \mathrm{MHz}, \mathrm{CDCl}_{3}, 300 \mathrm{~K}\right): \delta(\mathrm{ppm})=7.28(\mathrm{~s}, 4 \mathrm{H})$, $6.81(\mathrm{~d}, J=16.2 \mathrm{~Hz}, 1 \mathrm{H}), 6.13\left(\mathrm{dt}, J^{1}=16.2 \mathrm{~Hz}, J^{2}=2.2 \mathrm{~Hz}, 1 \mathrm{H}\right), 2.35\left(\mathrm{td}, J^{1}=7.0, J^{2}\right.$ $=1.9 \mathrm{~Hz}, 2 \mathrm{H}), 1.68-1.51\left(\mathrm{tq}, J^{1}=7.2 \mathrm{~Hz}, J^{2}=7.5 \mathrm{~Hz}, 2 \mathrm{H}\right), 1.02(\mathrm{t}, J=7.4 \mathrm{~Hz}, 3 \mathrm{H})$; ${ }^{13} \mathrm{C} \mathrm{NMR}\left(75 \mathrm{MHz}, \mathrm{CDCl}_{3}, 300 \mathrm{~K}\right): \delta(\mathrm{ppm})=138.6(\mathrm{CH}), 135.1(\mathrm{C}), 133.9(\mathrm{C}), 128.8$ $(\mathrm{CH}), 127.2(\mathrm{CH}), 109.6(\mathrm{CH}), 93.5(\mathrm{C}), 79.6(\mathrm{C}), 22.2\left(\mathrm{CH}_{2}\right), 21.6\left(\mathrm{CH}_{2}\right), 13.5\left(\mathrm{CH}_{3}\right)$; HRMS (ESI) $m / z=310.97512$ calcd. for $\mathrm{C}_{13} \mathrm{H}_{13} \mathrm{ClAg}[\mathrm{M}+\mathrm{Ag}]^{+}$, found: 310.97507 ; IR (neat, $\mathrm{cm}^{-1}$ ): 3033w, 2964m, 2872w, 2211w, 2159w, 1616w, 1490s, 1338w, 1277m, $1226 m, 1178 w, 1091 s, 1012 m, 954 s, 852 m, 806 s, 745 w, 682 w$.

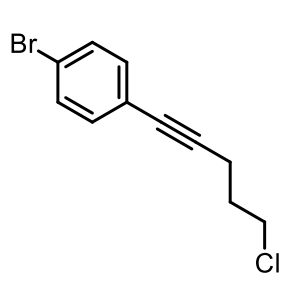

1-Bromo-4-(5-chloropent-1-yn-1-yl)benzene (3q): The title compound was prepared according to general procedure (GP1) with $\mathrm{Pd}\left(\mathrm{PPh}_{3}\right)_{2} \mathrm{Cl}_{2}(56.2 \mathrm{mg}, 80.0 \mu \mathrm{mol}, 1.0 \mathrm{~mol} \%), \mathrm{CuI}(30.5 \mathrm{mg}, 0.160$ mmol, $2.0 \mathrm{~mol} \%$ ), 1-bromo-4-iodobenzene (3.395 g, $12.00 \mathrm{mmol}$, 1.5 equiv), and 5-chloropent-1-yne ( $0.820 \mathrm{~g}, 8.00 \mathrm{mmol}, 1.0$ equiv) in $\mathrm{Et}_{3} \mathrm{~N}(8 \mathrm{~mL})$ at room temperature for 12 hours. Purification via silica gel chromatography (Pentane) gave the desired product $\mathbf{3 q}$ as a light yellow oil in $36 \%$ yield $(0.732 \mathrm{~g})$. TLC $\mathbf{R}_{\mathbf{f}}=0.45$ (Pentane); ${ }^{1} \mathbf{H}$ NMR $\left(300 \mathrm{MHz}, \mathrm{CDCl}_{3}, 300 \mathrm{~K}\right): \delta(\mathrm{ppm})=7.35(\mathrm{~d}, J=8.6 \mathrm{~Hz}, 2 \mathrm{H})$, $7.18(\mathrm{~d}, J=8.4 \mathrm{~Hz}, 2 \mathrm{H}), 3.63(\mathrm{t}, J=6.4 \mathrm{~Hz}, 2 \mathrm{H}), 2.53(\mathrm{t}, J=6.8 \mathrm{~Hz}, 2 \mathrm{H}), 1.98(\mathrm{p}, J=$ $6.6 \mathrm{~Hz}, 2 \mathrm{H}) ;{ }^{13} \mathrm{C}$ NMR $\left(75 \mathrm{MHz}, \mathrm{CDCl}_{3}, 300 \mathrm{~K}\right): \delta(\mathrm{ppm})=133.1(\mathrm{CH}), 131.5(\mathrm{CH})$, 122.6 (C), $121.9(\mathrm{C}), 89.4(\mathrm{C}), 80.5(\mathrm{C}), 43.7\left(\mathrm{CH}_{2}\right), 31.3\left(\mathrm{CH}_{2}\right), 16.9\left(\mathrm{CH}_{2}\right)$; EI-MS ( $m / z$, relative intensity): $258\left(\mathrm{M}+2^{+}, 100\right), 256\left(\mathrm{M}^{+}, 100\right), 220(71), 193(33), 162(8)$, 
149 (10); IR (neat, $\mathrm{cm}^{-1}$ ): 2959w, 2230w, 1901w, 1646w, 1586w, 1485s, 1438w, 1394w, $1352 w, 1290 m, 1070 s, 1010 s, 969 w, 823 s, 785 w, 723 w, 656 m$.

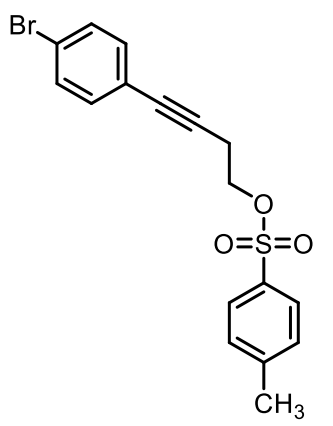

\section{4-(4-Bromophenyl)but-3-yn-1-yl 4-methylbenzenesulfonate}

(3r): The title compound was prepared according to general procedure (GP1) with $\mathrm{Pd}\left(\mathrm{PPh}_{3}\right)_{2} \mathrm{Cl}_{2}(56.2 \mathrm{mg}, 80.0 \mu \mathrm{mol}, 1.0$ = $=$ =o mol\%), CuI (30.5 mg, $0.160 \mathrm{mmol}, 2.0 \mathrm{~mol} \%)$, 1-bromo-4iodobenzene (3.40 g, $12.0 \mathrm{mmol}, 1.5$ equiv), and but-3-yn-1-yl 4methylbenzenesulfonate $\left(1.79 \mathrm{~g}, 8.0 \mathrm{mmol}, 1.0\right.$ equiv) in $\mathrm{Et}_{3} \mathrm{~N}$ (8 $\mathrm{mL}$ ) at room temperature for 12 hours. Purification via silica gel chromatography gave the desired product $3 \mathbf{r}$ as a light yellow solid in $51 \%$ yield $(1.530 \mathrm{~g})$. TLC $\mathbf{R}_{\mathrm{f}}=0.2$ $\left(\right.$ Pentane:EtOAc = 10:1); MP: $65{ }^{\mathbf{0}} \mathbf{C} ;{ }^{\mathbf{1}} \mathbf{H}$ NMR $\left(300 \mathrm{MHz}, \mathrm{CDCl}_{3}, 300 \mathrm{~K}\right): \delta(\mathrm{ppm})=$ $7.72(\mathrm{~d}, J=8.3 \mathrm{~Hz}, 2 \mathrm{H}), 7.31(\mathrm{~d}, J=8.5 \mathrm{~Hz}, 2 \mathrm{H}), 7.22(\mathrm{~d}, J=8.1 \mathrm{~Hz}, 2 \mathrm{H}), 7.09$ (d, $J=$ $8.5 \mathrm{~Hz}, 2 \mathrm{H}), 4.09(\mathrm{t}, J=6.9 \mathrm{~Hz}, 2 \mathrm{H}), 2.67(\mathrm{t}, J=6.9 \mathrm{~Hz}, 2 \mathrm{H}), 2.32(\mathrm{~s}, 3 \mathrm{H}) ;{ }^{13} \mathbf{C} \mathbf{N M R}$ $\left(75 \mathrm{MHz}, \mathrm{CDCl}_{3}, 300 \mathrm{~K}\right): \delta(\mathrm{ppm})=144.9(\mathrm{C}), 133.0(\mathrm{CH}), 132.7(\mathrm{C}), 131.4(\mathrm{CH})$, $129.8(\mathrm{CH}), 127.8(\mathrm{CH}), 122.2(\mathrm{C}), 121.8(\mathrm{C}), 85.2(\mathrm{C}), 81.5(\mathrm{C}), 67.5\left(\mathrm{CH}_{2}\right), 21.5$ $\left(\mathrm{CH}_{3}\right), 20.3\left(\mathrm{CH}_{2}\right)$; HRMS (ESI) $m / z=402.9797$ calcd. for $\mathrm{C}_{17} \mathrm{H}_{15} \mathrm{BrO}_{3} \mathrm{SNa}[\mathrm{M}+\mathrm{Na}]^{+}$, found: 402.9790; IR (neat, $\mathrm{cm}^{-1}$ ): 2961w, 2178w, 1975w, 1598w, 1486m, 1361s, 1306w, $1176 s, 1071 w, 980 s, 903 m, 822 s, 767 m, 664 m$.

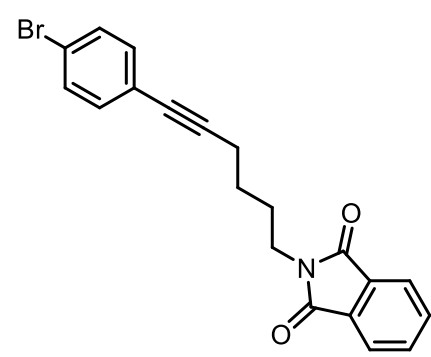

\section{2-(6-(4-Bromophenyl)hex-5-yn-1-yl)isoindoline-1,3-}

dione (3s): The title compound was prepared according to general procedure (GP1) with $\mathrm{Pd}\left(\mathrm{PPh}_{3}\right)_{2} \mathrm{Cl}_{2}(56.2 \mathrm{mg}$, $80.0 \mu \mathrm{mol}, 1.0 \mathrm{~mol} \%)$, CuI (30.5 mg, $0.160 \mathrm{mmol}, 2.0$ mol\%), 1-bromo-4-iodobenzene (3.40 g, $12.0 \mathrm{mmol}$, 1.5 equiv), and 2-(hex-5-yn-1-yl)isoindoline-1,3-dione (1.82 g, $8.0 \mathrm{mmol}, 1.0$ equiv) in $\mathrm{Et}_{3} \mathrm{~N}(15 \mathrm{~mL})$ at room temperature for 12 hours. Purification via silica gel chromatography gave the desired product $3 \mathrm{~s}$ as a brown solid in $83 \%$ yield (2.530 g). TLC $\mathbf{R}_{\mathbf{f}}=0.5$ (Pentane:EtOAc = 5:1); MP: $89{ }^{\circ} \mathrm{C} ;{ }^{1} \mathbf{H}$ NMR $\left(300 \mathrm{MHz}, \mathrm{CDCl}_{3}, 300 \mathrm{~K}\right)$ : $\delta(\mathrm{ppm})=7.76\left(\mathrm{dt}, J^{1}=4.8, J^{2}=3.6 \mathrm{~Hz}, 2 \mathrm{H}\right), 7.67-7.62(\mathrm{~m}, 2 \mathrm{H}), 7.32(\mathrm{~d}, J=7.9 \mathrm{~Hz}$, 
2H), $7.18\left(\mathrm{dd}, J^{1}=10.4 \mathrm{~Hz}, J^{2}=4.4 \mathrm{~Hz}, 2 \mathrm{H}\right), 3.68(\mathrm{t}, J=6.9 \mathrm{~Hz}, 2 \mathrm{H}), 2.38(\mathrm{t}, J=6.9$ $\mathrm{Hz}, 2 \mathrm{H}), 1.84-1.75(\mathrm{~m}, 2 \mathrm{H}), 1.59\left(\mathrm{dd}, J^{1}=15.1, J^{2}=7.4 \mathrm{~Hz}, 2 \mathrm{H}\right) ;{ }^{13} \mathbf{C}$ NMR $(75 \mathrm{MHz}$, $\left.\mathrm{CDCl}_{3}, 300 \mathrm{~K}\right): \delta(\mathrm{ppm})=168.4(\mathrm{C}), 133.9(\mathrm{CH}), 133.0(\mathrm{CH}), 132.1(\mathrm{C}), 131.4(\mathrm{CH})$, $123.2(\mathrm{CH}), 122.8(\mathrm{C}), 121.6(\mathrm{C}), 90.7(\mathrm{C}), 80.1(\mathrm{C}), 37.5\left(\mathrm{CH}_{2}\right), 27.8\left(\mathrm{CH}_{2}\right), 25.8$ $\left(\mathrm{CH}_{2}\right), 19.0\left(\mathrm{CH}_{2}\right)$; HRMS (ESI) $\mathrm{m} / z=404.0257$ calcd. for $\mathrm{C}_{20} \mathrm{H}_{16} \mathrm{BrNO}_{2} \mathrm{Na}[\mathrm{M}+\mathrm{Na}]^{+}$, found: 404.0246; IR (neat, $\mathrm{cm}^{-1}$ ): 2942w, 2237w, 1904w, 1771m, 1706s, 1615w, 1485w, $1436 w, 1395 s, 1188 w, 1116 w, 1037 m, 921 w, 825 m, 718 s$.

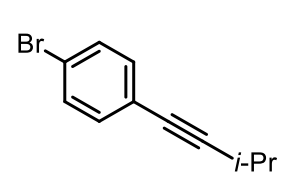

1-Bromo-4-(3-methylbut-1-yn-1-yl)benzene (3u): The title compound was prepared according to general procedure (GP1) with $\mathrm{Pd}\left(\mathrm{PPh}_{3}\right)_{2} \mathrm{Cl}_{2}(56.2 \mathrm{mg}, 80.0 \mu \mathrm{mol}, 1.0 \mathrm{~mol} \%), \mathrm{CuI}(30.5 \mathrm{mg}$, $0.160 \mathrm{mmol}, 2.0 \mathrm{~mol} \%$ ), 1-bromo-4-iodobenzene (3.40 g, $12.0 \mathrm{mmol}, 1.5$ equiv), and 3-methylbut-1-yne (0.545 g, $8.0 \mathrm{mmol}, 1.0$ equiv) in $\mathrm{Et}_{3} \mathrm{~N}(15 \mathrm{~mL})$ at room temperature for 12 hours. Purification via silica gel chromatography (Pentane) gave the desired product $3 \mathbf{u}$ as a yellow oil in $88 \%$ yield (1.562 g). $\mathbf{T L C} \mathbf{R}_{\mathrm{f}}=0.75$ (Pentane); ${ }^{1} \mathbf{H}$ NMR $\left(300 \mathrm{MHz}, \mathrm{CDCl}_{3}, 300 \mathrm{~K}\right): \delta(\mathrm{ppm})=7.33(\mathrm{~d}, J=8.5 \mathrm{~Hz}, 2 \mathrm{H}), 7.17(\mathrm{~d}, J=8.5 \mathrm{~Hz}, 2 \mathrm{H})$, 2.68 (hept, $J=6.9 \mathrm{~Hz}, 1 \mathrm{H}), 1.18(\mathrm{~d}, J=6.9 \mathrm{~Hz}, 6 \mathrm{H}) ;{ }^{13} \mathbf{C ~ N M R}\left(75 \mathrm{MHz}, \mathrm{CDCl}_{3}, 300\right.$ $\mathrm{K}): \delta(\mathrm{ppm})=133.0(\mathrm{CH}), 131.4(\mathrm{CH}), 123.0(\mathrm{C}), 121.5(\mathrm{C}), 97.0(\mathrm{C}), 78.8(\mathrm{C}), 22.9$ $\left(\mathrm{CH}_{3}\right), 21.1(\mathrm{CH})$; HRMS (ESI) $m / z=360.89674$ calcd. for $\mathrm{C}_{11} \mathrm{H}_{11} \mathrm{BrAgO}_{2}\left[\mathrm{M}+\mathrm{AgO}_{2}\right]^{+}$, found: 360.89740; IR (neat, $\mathrm{cm}^{-1}$ ): 3091w, 2964w, 2925w, 2869w, 2227w, 1642w, $1424 s, 1212 s, 1132 s, 1072 m, 1000 s, 837 s, 762 w, 735 w, 686 w, 655 w$.

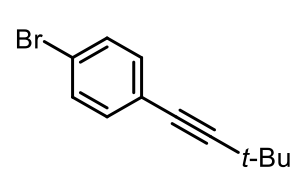

1-Bromo-4-(3,3-dimethylbut-1-yn-1-yl)benzene (3v): The title compound was prepared according to general procedure (GP1) with $\mathrm{Pd}\left(\mathrm{PPh}_{3}\right)_{2} \mathrm{Cl}_{2}(56.2 \mathrm{mg}, 80.0 \mu \mathrm{mol}, 1.0 \mathrm{~mol} \%), \mathrm{CuI}(30.5 \mathrm{mg}$, $0.160 \mathrm{mmol}, 2.0 \mathrm{~mol} \%$ ), 1-bromo-4-iodobenzene (3.40 g, $12.0 \mathrm{mmol}, 1.5$ equiv), and 3,3-dimethylbut-1-yne (0.657 $\mathrm{g}, 8.0 \mathrm{mmol}, 1.0$ equiv) in $\mathrm{Et}_{3} \mathrm{~N}(15 \mathrm{~mL})$ at room temperature for 12 hours. Purification via silica gel chromatography (Pentane) gave the desired product $3 \mathrm{v}$ as a white solid in $85 \%$ yield (1.617 g). TLC $\mathbf{R}_{\mathrm{f}}=0.75$ (Pentane); MP: $50{ }^{\circ} \mathrm{C} ;{ }^{1} \mathbf{H}$ NMR $\left(300 \mathrm{MHz}, \mathrm{CDCl}_{3}, 300 \mathrm{~K}\right): \delta(\mathrm{ppm})=7.32(\mathrm{~d}, J=8.6 \mathrm{~Hz}, 2 \mathrm{H})$, 
$7.16(\mathrm{~d}, J=8.6 \mathrm{~Hz}, 2 \mathrm{H}), 1.23(\mathrm{~s}, 9 \mathrm{H}) ;{ }^{13} \mathbf{C ~ N M R}\left(75 \mathrm{MHz}, \mathrm{CDCl}_{3}, 300 \mathrm{~K}\right): \delta(\mathrm{ppm})=$ $133.0(\mathrm{CH}), 131.3(\mathrm{CH}), 123.1(\mathrm{C}), 121.4(\mathrm{C}), 99.7(\mathrm{C}), 78.09(\mathrm{C}), 30.9\left(\mathrm{CH}_{3}\right), 28.0$ (C); EI-MS ( $\mathrm{m} / \mathrm{z}$, relative intensity): $236\left(\mathrm{M}^{+}, 24\right), 221$ (47), 157 (14), 142 (100), 141 (75), 126 (22), 115 (44), 102 (18), 77 (17), 71 (16), 63 (12); IR (neat, $\mathrm{cm}^{-1}$ ): 2989s, $2236 w, 1484 s, 1392 w, 1362 m, 1291 s, 1203 w, 1071 s, 1010 s, 916 w, 823 s$.

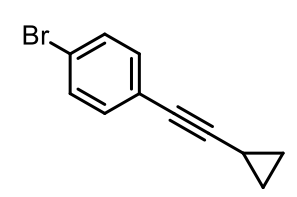

1-Bromo-4-(cyclopropylethynyl)benzene (3w): The title compound was prepared according to general procedure (GP1) with $\mathrm{Pd}\left(\mathrm{PPh}_{3}\right)_{2} \mathrm{Cl}_{2}(56.2 \mathrm{mg}, 80.0 \mu \mathrm{mol}, 1.0 \mathrm{~mol} \%), \mathrm{CuI}(30.5 \mathrm{mg}, 0.160$ mmol, $2.0 \mathrm{~mol} \%$ ), 1-bromo-4-iodobenzene (3.40 g, $12.0 \mathrm{mmol}, 1.5$ equiv), and ethynylcyclopropane $\left(0.53 \mathrm{~g}, 8.0 \mathrm{mmol}, 1.0\right.$ equiv) in $\mathrm{Et}_{3} \mathrm{~N}(15 \mathrm{~mL})$ at room temperature for 12 hours. Purification via silica gel chromatography (Pentane) gave the desired product 3w as a white solid in $75 \%$ yield (1.314 g). TLC $\mathbf{R}_{\mathrm{f}}=0.7$ (Pentane); MP: $32{ }^{\circ} \mathrm{C} ;{ }^{1} \mathbf{H}$ NMR $\left(300 \mathrm{MHz}, \mathrm{CDCl}_{3}, 300 \mathrm{~K}\right): \delta(\mathrm{ppm})=7.31(\mathrm{~d}, J=8.4 \mathrm{~Hz}, 2 \mathrm{H})$, $7.15(\mathrm{~d}, J=8.4 \mathrm{~Hz}, 2 \mathrm{H}), 1.38-1.31(\mathrm{~m}, 1 \mathrm{H}), 0.95-0.67(\mathrm{~m}, 4 \mathrm{H}) ;{ }^{13} \mathbf{C} \mathbf{N M R}(75 \mathrm{MHz}$, $\left.\mathrm{CDCl}_{3}, 300 \mathrm{~K}\right): \delta(\mathrm{ppm})=133.0(\mathrm{CH}), 131.4(\mathrm{CH}), 122.9(\mathrm{C}), 121.5(\mathrm{C}), 94.7(\mathrm{C}), 74.8$ (C), $8.6\left(\mathrm{CH}_{2}\right), 0.2(\mathrm{CH})$; EI-MS (m/z, relative intensity): $220\left(\mathrm{M}^{+}, 18\right), 141(48), 139$ (19), 115 (100), 113 (39), 101 (5), 87 (16), 74 (10), 70 (12), 63 (21), 50 (8); IR (neat, $\left.\mathrm{cm}^{-1}\right): 3012 w, 2233 w, 1899 w, 1782 w, 1645 w, 1587 w, 1485 s, 1393 w, 1361 w, 1179 w$ $1069 s, 1028 w, 953 s, 822 s$.

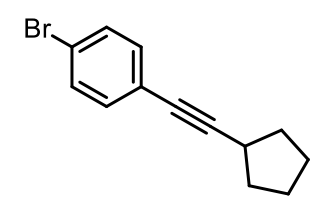

1-Bromo-4-(cyclopentylethynyl)benzene $\quad(3 x):$ The title compound was prepared according to general procedure (GP1) with $\mathrm{Pd}\left(\mathrm{PPh}_{3}\right)_{2} \mathrm{Cl}_{2}(56.2 \mathrm{mg}, 80.0 \mu \mathrm{mol}, 1.0 \mathrm{~mol} \%), \mathrm{CuI}(30.5 \mathrm{mg}$, $0.160 \mathrm{mmol}, 2.0 \mathrm{~mol} \%$ ), 1-bromo-4-iodobenzene (3.40 g, $12.0 \mathrm{mmol}, 1.5$ equiv), and ethynylcyclopentane $\left(0.753 \mathrm{~g}, 8.0 \mathrm{mmol}, 1.0\right.$ equiv) in $\mathrm{Et}_{3} \mathrm{~N}(15 \mathrm{~mL})$ at room temperature for 12 hours. Purification via silica gel chromatography (Pentane) gave the desired product 3x as a white solid in $84 \%$ yield $(1.667 \mathrm{~g})$. TLC $\mathbf{R}_{\mathrm{f}}=0.85$ (Pentane); MP: $29{ }^{\circ} \mathrm{C} ;{ }^{1} \mathbf{H}$ NMR $\left(300 \mathrm{MHz}, \mathrm{CDCl}_{3}, 300 \mathrm{~K}\right): \delta(\mathrm{ppm})=7.32(\mathrm{~d}, J=8.5 \mathrm{~Hz}, 2 \mathrm{H})$, $7.17(\mathrm{~d}, J=8.5 \mathrm{~Hz}, 2 \mathrm{H}), 2.73(\mathrm{p}, J=7.5 \mathrm{~Hz}, 1 \mathrm{H}), 1.97-1.86(\mathrm{~m}, 2 \mathrm{H}), 1.77-1.51(\mathrm{~m}$, 
6H); ${ }^{13} \mathrm{C} \mathrm{NMR}\left(75 \mathrm{MHz}, \mathrm{CDCl}_{3}, 300 \mathrm{~K}\right): \delta(\mathrm{ppm})=133.0(\mathrm{CH}), 131.4(\mathrm{CH}), 123.2$ (C), $121.4(\mathrm{C}), 95.9(\mathrm{C}), 79.1(\mathrm{C}), 33.8\left(\mathrm{CH}_{2}\right), 30.8(\mathrm{CH}), 25.1\left(\mathrm{CH}_{2}\right)$; EI-MS $(\mathrm{m} / \mathrm{z}$, relative intensity): $248\left(\mathrm{M}^{+}, 32\right), 221$ (13), 169 (25), 154 (19), 141 (100), 127 (22), 115 (17), 101 (11), 91 (11), 77 (10), 63 (12), 41 (10); IR (neat, $\mathrm{cm}^{-1}$ ): 2961s, 2870m, 2225w, $1911 w, 1644 w, 1486 s, 1393 w, 1341 w, 1300 w, 1071 s, 1010 s, 941 w, 991 w, 823 s, 703 w$.

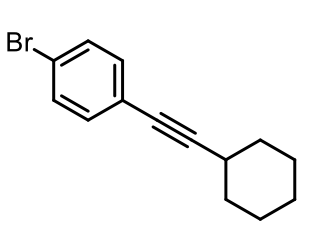

1-Bromo-4-(cyclohexylethynyl)benzene $\quad(\mathbf{3 y}): \quad$ The title compound was prepared according to general procedure (GP1) with $\mathrm{Pd}\left(\mathrm{PPh}_{3}\right)_{2} \mathrm{Cl}_{2}(70.2 \mathrm{mg}, 0.100 \mathrm{mmol}, 1.0 \mathrm{~mol} \%)$, $\mathrm{CuI}(38.1 \mathrm{mg}$, $0.200 \mathrm{mmol}, 2.0 \mathrm{~mol} \%$ ), 1-bromo-4-iodobenzene (3.40 g, 12.0 mmol, 1.2 equiv), and ethynylcyclohexane (1.08 g, $10.0 \mathrm{mmol}, 1.0$ equiv) in $\mathrm{Et}_{3} \mathrm{~N}$ (15 $\mathrm{mL})$ at room temperature for 12 hours. Purification via silica gel chromatography (Pentane) gave the desired product 3y as a light yellow solid in 95\% yield (2.495 g). TLC $\mathbf{R}_{\mathrm{f}}=0.85$ (Pentane); MP: $62{ }^{\circ} \mathrm{C} ;{ }^{1} \mathbf{H}$ NMR $\left(300 \mathrm{MHz}, \mathrm{CDCl}_{3}, 300 \mathrm{~K}\right): \delta(\mathrm{ppm})=$ $7.32(\mathrm{~d}, J=8.4 \mathrm{~Hz}, 2 \mathrm{H}), 7.17(\mathrm{~d}, J=8.3 \mathrm{~Hz}, 2 \mathrm{H}), 2.49\left(\mathrm{ddd}, J^{1}=12.7 \mathrm{~Hz}, J^{2}=8.8 \mathrm{~Hz}\right.$, $\left.J^{3}=3.6 \mathrm{~Hz}, 1 \mathrm{H}\right), 1.81-1.77(\mathrm{~m}, 2 \mathrm{H}), 1.67\left(\mathrm{dd}, J^{1}=8.9 \mathrm{~Hz}, J^{2}=3.7 \mathrm{~Hz}, 2 \mathrm{H}\right), 1.45(\mathrm{dd}$, $\left.J^{1}=22.3 \mathrm{~Hz}, J^{2}=8.9 \mathrm{~Hz}, 3 \mathrm{H}\right), 1.27-1.21(\mathrm{~m}, 3 \mathrm{H}) ;{ }^{13} \mathbf{C ~ N M R}\left(75 \mathrm{MHz}, \mathrm{CDCl}_{3}, 300 \mathrm{~K}\right)$ : $\delta(\mathrm{ppm})=133.0(\mathrm{CH}), 131.3(\mathrm{CH}), 123.2(\mathrm{C}), 121.4(\mathrm{C}), 95.7(\mathrm{C}), 79.5(\mathrm{C}), 32.6\left(\mathrm{CH}_{2}\right)$, $29.7(\mathrm{CH}), 25.9\left(\mathrm{CH}_{2}\right), 24.9\left(\mathrm{CH}_{2}\right)$; EI-MS ( $\mathrm{m} / z$, relative intensity): $264\left(\mathrm{M}+2^{+}, 48\right)$, $262\left(\mathrm{M}^{+}, 47\right), 221$ (21), 183 (21), 154 (52), 141 (100), 127 (44), 115 (25), 77 (28), 41 (28); IR (neat, $\mathrm{cm}^{-1}$ ): 2929s, 2854s, 2230w, 1485s, 1448m, 1393w, 1359w, 1300w, $1257 w, 1070 s, 1011 s, 952 w, 888 s, 745 w$.
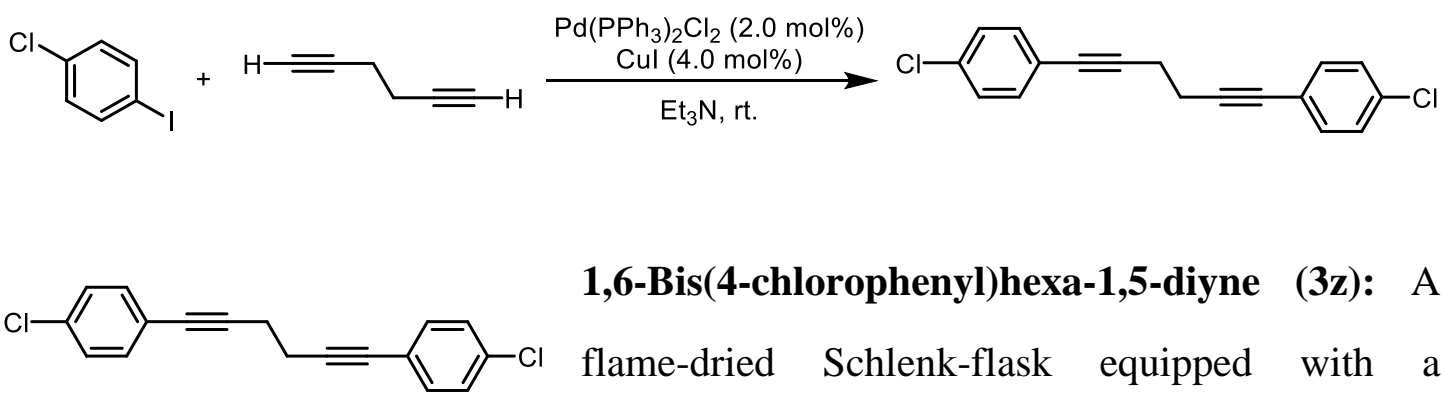

1,6-Bis(4-chlorophenyl)hexa-1,5-diyne (3z): A

flame-dried Schlenk-flask equipped with a magnetic stir bar was charged with bis(triphenylphosphine)palladium(II) dichloride 
(180 mg, $0.256 \mathrm{mmol}, 2.0 \mathrm{~mol} \%$ ) and copper(I) iodide (97.6 mg, $0.512 \mathrm{mmol}, 4.0$ mol\%), 1-chloro-4-iodobenzene (7.332 g, $30.75 \mathrm{mmol}, 2.4$ equiv), sealed with a septum, and degassed by alternating vacuum evacuation and argon backfilling (three times) before triethylamine $(24 \mathrm{~mL})$ was added. 1,5-hexadiyne (50\% in pentane) $(1.000 \mathrm{~g}$, $12.81 \mathrm{mmol}, 1.0$ equiv) was added to the resulting suspension subsequently. The reaction mixture was then stirred at room temperature for 12 hours. After the reaction was complete, the reaction mixture was diluted with $\mathrm{Et}_{2} \mathrm{O}(30 \mathrm{~mL})$ and filtrated through a small pad of silica gel. The solvent was removed under reduced pressure with the aid of a rotary evaporator and the crude residue was purified by a silica gel column chromatography (Pentane, then Pentane:EtOAc $=250: 1$ ) to give pure 1,5 diynes $\mathbf{3 z}$ as a light yellow solid in 47\% yield (1.802 g). TLC $\mathbf{R}_{\mathrm{f}}=0.9$ (Pentane); MP: $148{ }^{\circ} \mathrm{C} ;{ }^{1} \mathbf{H}$ NMR $\left(300 \mathrm{MHz}, \mathrm{CDCl}_{3}, 300 \mathrm{~K}\right): \delta(\mathrm{ppm})=7.26(\mathrm{~d}, J=8.6 \mathrm{~Hz}, 4 \mathrm{H}), 7.18(\mathrm{~d}, J=8.7$ $\mathrm{Hz}, 4 \mathrm{H}), 2.63(\mathrm{~s}, 4 \mathrm{H}) ;{ }^{13} \mathrm{C}$ NMR $\left(75 \mathrm{MHz}, \mathrm{CDCl}_{3}, 300 \mathrm{~K}\right): \delta(\mathrm{ppm})=133.8(\mathrm{C}), 132.8$ (CH), $128.5(\mathrm{CH}), 122.1(\mathrm{C}), 89.2(\mathrm{C}), 80.6(\mathrm{C}), 19.7\left(\mathrm{CH}_{2}\right)$; HRMS (ESI) $\mathrm{m} / \mathrm{z}=$ 404.93615 calcd. for $\mathrm{C}_{18} \mathrm{H}_{12} \mathrm{Cl}_{2} \mathrm{Na}[\mathrm{M}+\mathrm{Na}]^{+}$, found: 404.93621; IR (neat, $\mathrm{cm}^{-1}$ ): $2916 w$, $2001 w, 1912 w, 1663 w, 1591 w, 1488 s, 1438 w, 1397 w, 1274 w, 1095 s, 1015 m, 830 s$, $744 w$.

\section{Regio- and stereoselective cyanotriflation of alkynes using aryl(cyano)- iodonium triflates}

\section{General procedure for cyanotriflation of alkynes (GP2)}

A flame-dried Schlenk-tube equipped with a magnetic stir bar was charged with iron(II) acetate $(3.5 \mathrm{mg}, 0.020 \mathrm{mmol}, 10 \mathrm{mmol} \%)$ and 1,10-phenanthroline (3.6 mg, $0.020 \mathrm{mmol}, 10 \mathrm{mmol} \%$ ), sealed with a septum, and degassed by alternating vacuum evacuation and argon backfilling (three times) before DCE $(1 \mathrm{~mL})$ was added. The resulting suspension was stirred for $5 \mathrm{~min}$ at room temperature. The corresponding alkyne 3 (0.200 mmol, 1.0 equiv) and 3,5-di(trifluoromethyl)phenyl(cyano)iodonium triflate 1a (227 mg, $0.440 \mathrm{mmol}, 2.2$ equiv) were added successively under a flow of argon. The reaction mixture was then stirred at $45^{\circ} \mathrm{C}$ for $15 \mathrm{~h}$. After the reaction was complete, the solvent was removed under reduced pressure with the aid of a rotary 
evaporator. The crude residue was purified by silica gel column chromatography to afford pure cyanotriflated product 2 .

\section{Scale-up experiment}

A flame-dried Schlenk-tube equipped with a magnetic stir bar was charged with iron(II) acetate $(87.0 \mathrm{mg}, 0.500 \mathrm{mmol}, 10 \mathrm{mmol} \%)$ and 1,10-phenanthroline (90.1 mg, $0.500 \mathrm{mmol}, 10 \mathrm{mmol} \%$ ), sealed with a septum, and degassed by alternating vacuum evacuation and argon backfilling (three times) before DCE $(25 \mathrm{~mL})$ was added. The resulting suspension was stirred for $5 \mathrm{~min}$ at room temperature. The corresponding alkyne 1-bromo-4-(pent-1-yn-1-yl)benzene 3e (1.116 g, $5.000 \mathrm{mmol}, 1.0$ equiv) and 3,5-di(trifluoromethyl)phenyl(cyano)iodonium triflate 1a (5.664 g, $11.00 \mathrm{mmol}, 2.2$ equiv) were added successively under a flow of argon. The reaction mixture was then stirred at $45{ }^{\circ} \mathrm{C}$ for 24 hours. After the reaction was complete, the solvent was removed under reduced pressure with the aid of a rotary evaporator. The crude residue was purified by silica gel column chromatography (Pentane:EtOAc $=200: 1)$ to afford pure cyanotriflated product $\mathbf{2 e}$ as a light yellow oil in $88 \%$ yield (1.741 $\mathrm{g}, \mathrm{dr}>20: 1)$; $\mathbf{T L C} \mathbf{R}_{\mathrm{f}}$ $=0.50($ Pentane:EtOAc $=20: 1)$.

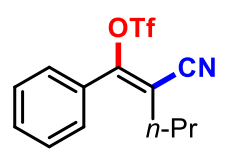

\section{(Z)-2-Cyano-1-phenylpent-1-en-1-yl trifluoromethanesulfonate (2a):}

The title compound was prepared according to general procedure (GP2) with $\mathrm{Fe}(\mathrm{OAc})_{2}(3.5 \mathrm{mg}, 20 \mu \mathrm{mol}, 10 \mathrm{~mol} \%)$, L1 (3.6 mg, $20 \mu \mathrm{mol}, 10$ mol\%), pent-1-yn-1-ylbenzene 3a (28.8 mg, $0.200 \mathrm{mmol}, 1.0$ equiv), and 3,5di(trifluoromethyl)phenyl(cyano)iodonium triflate 1a (227 mg, $0.440 \mathrm{mmol}, 2.2$ equiv) in DCE $(1 \mathrm{~mL})$ at $45{ }^{\circ} \mathrm{C}$ for $15 \mathrm{~h}$. Purification via silica gel chromatography gave the desired product $2 \mathbf{a}$ as a yellow oil in $40 \%$ yield $(25.5 \mathrm{mg})$. TLC $\mathbf{R}_{\mathrm{f}}=0.50$ $($ Pentane:EtOAc $=20: 1) ;{ }^{1} \mathbf{H}$ NMR $\left(300 \mathrm{MHz}, \mathrm{CDCl}_{3}, 300 \mathrm{~K}\right): \delta(\mathrm{ppm})=7.77-7.31$ $(\mathrm{m}, 5 \mathrm{H}), 2.24(\mathrm{t}, J=7.5 \mathrm{~Hz}, 2 \mathrm{H}), 1.62\left(\mathrm{tq}, J^{1}=7.5 \mathrm{~Hz}, J^{2}=7.5 \mathrm{~Hz}, 2 \mathrm{H}\right), 0.86(\mathrm{t}, J=7.4$ $\mathrm{Hz}, 3 \mathrm{H}) ;{ }^{13} \mathbf{C ~ N M R}\left(75 \mathrm{MHz}, \mathrm{CDCl}_{3}, 300 \mathrm{~K}\right): \delta(\mathrm{ppm})=155.6(\mathrm{C}), 131.8(\mathrm{CH}), 129.8$ (C), $129.0(\mathrm{CH}), 128.8(\mathrm{CH}), 118.1$ (q, $\left.J=320.9 \mathrm{~Hz}, \mathrm{CF}_{3}\right), 114.3(\mathrm{C}), 109.5(\mathrm{C}), 31.4$ $\left(\mathrm{CH}_{2}\right), 21.4\left(\mathrm{CH}_{2}\right), 13.2\left(\mathrm{CH}_{3}\right) ;{ }^{19} \mathbf{F}$ NMR $\left(282 \mathrm{MHz}, \mathrm{CDCl}_{3}, 300 \mathrm{~K}\right): \delta(\mathrm{ppm})=-73.5$ 
(s, 3F); HRMS (ESI) $m / z=342.0382$, calcd. for $\mathrm{C}_{13} \mathrm{H}_{12} \mathrm{~F}_{3} \mathrm{NO}_{3} \mathrm{SNa}[\mathrm{M}+\mathrm{Na}]^{+}$, found: 342.0385; IR (neat, $\mathrm{cm}^{-1}$ ): 2970w, 2878w, 2226w, 1644w, 1426s, 1216s, 1136s, 1093w, $1000 m, 929 w, 859 w, 820 m, 770 w, 698 w$.<smiles>C=C(P)C(C#N)=C(OBr)c1ccc(C)cc1</smiles>

\section{(Z)-2-Cyano-1-(p-tolyl)pent-1-en-1-yl trifluoromethanesulfon-}

ate (2b): The title compound was prepared according to general procedure (GP2) with $\mathrm{Fe}(\mathrm{OAc})_{2}(3.5 \mathrm{mg}, 20 \mu \mathrm{mol}, 10 \mathrm{~mol} \%), \mathbf{L 1}$ (3.6 mg, $20 \mu \mathrm{mol}, 10$ mol\%), 1-methyl-4-(pent-1-yn-1-yl)benzene 3b (31.6 mg, 0.200 mmol, 1.0 equiv), and 3,5-di(trifluoromethyl)phenyl(cyano)iodonium triflate 1a (227 $\mathrm{mg}, 0.440 \mathrm{mmol}, 2.2$ equiv) in DCE $(1 \mathrm{~mL})$ at $45^{\circ} \mathrm{C}$ for $15 \mathrm{~h}$. Purification via silica gel chromatography gave the desired product $\mathbf{2 b}$ as a light yellow oil in $41 \%$ yield $(27.1$ mg). TLC $\mathbf{R}_{\mathrm{f}}=0.50$ (Pentane:EtOAc $\left.=20: 1\right) ;{ }^{1} \mathbf{H} \mathbf{N M R}\left(300 \mathrm{MHz}, \mathrm{CDCl}_{3}, 300 \mathrm{~K}\right): \delta$ $(\mathrm{ppm})=7.23(\mathrm{~m}, 4 \mathrm{H}), 2.34(\mathrm{~s}, 3 \mathrm{H}), 2.23(\mathrm{t}, J=7.5 \mathrm{~Hz}, 2 \mathrm{H}), 1.60\left(\mathrm{tq}, J^{1}=7.5 \mathrm{~Hz}, J^{2}=\right.$ $7.5 \mathrm{~Hz}, 2 \mathrm{H}), 0.85(\mathrm{t}, J=7.4 \mathrm{~Hz}, 3 \mathrm{H}) ;{ }^{13} \mathbf{C} \mathbf{N M R}\left(75 \mathrm{MHz}, \mathrm{CDCl}_{3}, 300 \mathrm{~K}\right): \delta(\mathrm{ppm})=$ $155.9(\mathrm{C}), 142.6(\mathrm{C}), 129.7(\mathrm{CH}), 128.7(\mathrm{CH}), 126.9(\mathrm{C}), 118.1$ (q, J = 320.9 Hz, $\left.\mathrm{CF}_{3}\right)$, $114.5(\mathrm{C}), 108.7(\mathrm{C}), 31.4\left(\mathrm{CH}_{2}\right), 21.5\left(\mathrm{CH}_{3}\right), 21.4\left(\mathrm{CH}_{2}\right), 13.1\left(\mathrm{CH}_{3}\right) ;{ }^{19}$ F NMR (282 $\left.\mathrm{MHz}_{\mathrm{CDCl}}, 300 \mathrm{~K}\right): \delta(\mathrm{ppm})=-73.6(\mathrm{~s}, 3 \mathrm{~F})$; HRMS (ESI) $\mathrm{m} / \mathrm{z}=356.0539$, calcd. for $\mathrm{C}_{14} \mathrm{H}_{14} \mathrm{~F}_{3} \mathrm{NO}_{3} \mathrm{SNa}[\mathrm{M}+\mathrm{Na}]^{+}$, found: 356.0552; IR (neat, $\mathrm{cm}^{-1}$ ): 2969w, 2257w, 2227w, $1644 w, 1426 w, 1218 m, 1136 m, 1092 w, 997 m, 905 s, 830 w, 725 s, 649 w, 607 w$.<smiles></smiles>

(Z)-1-(4-Chlorophenyl)-2-cyanopent-1-en-1-yl trifluoromethanesulfonate (2c): The title compound was prepared according to general procedure (GP2) with $\mathrm{Fe}(\mathrm{OAc})_{2}(3.5 \mathrm{mg}, 20 \mu \mathrm{mol}, 10 \mathrm{~mol} \%)$, L1 (3.6 mg, $20 \mu \mathrm{mol}, 10$ mol\%), 1-chloro-4-(pent-1-yn-1-yl)benzene 3c (35.7 mg, 0.200 mmol, 1.0 equiv), and 3,5-di(trifluoromethyl)phenyl(cyano)iodonium triflate 1a (227 mg, $0.440 \mathrm{mmol}, 2.2$ equiv) in DCE (1 mL) at $45{ }^{\circ} \mathrm{C}$ for $15 \mathrm{~h}$. Purification via silica gel chromatography (Pentane:EtOAc $=300: 1)$ gave the desired product $\mathbf{2 c}$ as a light yellow oil in $81 \%$ yield $(57.1 \mathrm{mg}) . \mathbf{T L C} \mathbf{R}_{\mathrm{f}}=0.35$ (Pentane:EtOAc $\left.=20: 1\right) ;{ }^{1} \mathbf{H}$ NMR $\left(300 \mathrm{MHz}, \mathrm{CDCl}_{3}, 300 \mathrm{~K}\right): \delta(\mathrm{ppm})=7.41(\mathrm{~d}, J=8.6 \mathrm{~Hz}, 2 \mathrm{H}), 7.31(\mathrm{~d}, J=8.7$ $\mathrm{Hz}, 2 \mathrm{H}), 2.21(\mathrm{t}, J=7.5 \mathrm{~Hz}, 2 \mathrm{H}), 1.60\left(\mathrm{tq}, J^{1}=7.5 \mathrm{~Hz}, J^{2}=7.5 \mathrm{~Hz}, 2 \mathrm{H}\right), 0.86(\mathrm{t}, J=7.4$ 
$\mathrm{Hz}, 3 \mathrm{H}) ;{ }^{13} \mathbf{C}$ NMR $\left(75 \mathrm{MHz}, \mathrm{CDCl}_{3}, 300 \mathrm{~K}\right): \delta(\mathrm{ppm})=154.3(\mathrm{C}), 138.3(\mathrm{C}), 130.1$ (CH), 129.5 (CH), 128.1 (C), 118.1 (q, $J=320.9$ Hz, $\mathrm{CF}_{3}$ ), $114.0(\mathrm{C}), 110.1(\mathrm{C}), 31.4$ $\left(\mathrm{CH}_{2}\right), 21.3\left(\mathrm{CH}_{2}\right), 13.1\left(\mathrm{CH}_{3}\right) ;{ }^{19} \mathbf{F} \mathbf{N M R}\left(282 \mathrm{MHz}, \mathrm{CDCl}_{3}, 300 \mathrm{~K}\right): \delta(\mathrm{ppm})=-73.4$ (s, 3F); HRMS (ESI) $m / z=375.9992$, calcd. for $\mathrm{C}_{13} \mathrm{H}_{11} \mathrm{ClF}_{3} \mathrm{NO}_{3} \mathrm{SNa}[\mathrm{M}+\mathrm{Na}]^{+}$, found: 375.9990; IR (neat, $\mathrm{cm}^{-1}$ ): 2969w, 2938w, 2878w, 2227w, 1644w, 1594w, 1489w, $1425 s, 1346 w, 1211 s, 1133 s, 1093 s, 997 s, 914 w, 838 s, 811 s, 763 w, 739 s, 686 w$.

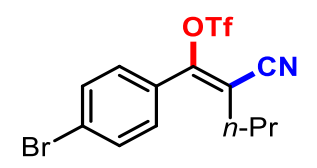

(Z)-1-(4-Chloro-3-methylphenyl)-2-cyanopent-1-en-1-yl tri

fluoromethanesulfonate (2d): The title compound was prepared according to general procedure (GP2) with $\mathrm{Fe}(\mathrm{OAc})_{2}(3.5 \mathrm{mg}, 20$ $\mu \mathrm{mol}, 10$ mol\%), L1 (3.6 mg, 20 mol, 10 mol\%), 1-chloro-2-methyl-4-(pent-1-yn-1yl)benzene $\quad 3 d \quad(38.4 \quad \mathrm{mg}, \quad 0.200 \quad \mathrm{mmol}, \quad 1.0 \quad$ equiv), and 3,5di(trifluoromethyl)phenyl(cyano)iodonium triflate 1a $(227 \mathrm{mg}, 0.440 \mathrm{mmol}, 2.2$ equiv) in DCE $(1 \mathrm{~mL})$ at $45^{\circ} \mathrm{C}$ for $15 \mathrm{~h}$. Purification via silica gel chromatography (Pentane:EtOAc $=400: 1$ ) gave the desired product 2d as a slight yellow oil in 76\% yield (55.7 mg). $\mathbf{T L C} \mathbf{R}_{\mathrm{f}}=0.5$ (Pentane:EtOAc $\left.=20: 1\right) ;{ }^{1} \mathbf{H} \mathbf{~ N M R}\left(300 \mathrm{MHz}, \mathrm{CDCl}_{3}\right.$, $300 \mathrm{~K}): \delta(\mathrm{ppm})=7.40(\mathrm{~d}, J=8.3 \mathrm{~Hz}, 1 \mathrm{H}), 7.23(\mathrm{~d}, J=1.7 \mathrm{~Hz}, 1 \mathrm{H}), 7.14\left(\mathrm{dd}, J^{1}=8.3\right.$ $\left.\mathrm{Hz}, J^{2}=1.9 \mathrm{~Hz}, 1 \mathrm{H}\right), 2.36(\mathrm{~s}, 1 \mathrm{H}), 2.27-2.15(\mathrm{t}, J=7.5 \mathrm{~Hz}, 2 \mathrm{H}), 1.61$ (tq, $J^{1}=7.5 \mathrm{~Hz}$, $\left.J^{2}=7.2 \mathrm{~Hz}, 2 \mathrm{H}\right), 0.87(\mathrm{t}, J=7.4 \mathrm{~Hz}, 3 \mathrm{H}) .{ }^{13} \mathbf{C ~ N M R}\left(75 \mathrm{MHz}, \mathrm{CDCl}_{3}, 300 \mathrm{~K}\right): \delta(\mathrm{ppm})$ $=154.6(\mathrm{C}), 138.4(\mathrm{C}), 137.5(\mathrm{C}), 131.0(\mathrm{CH}), 129.9(\mathrm{CH}), 128.2(\mathrm{C}), 127.5(\mathrm{CH})$, $118.1\left(\mathrm{q}, J=321.0 \mathrm{~Hz}, \mathrm{CF}_{3}\right), 114.1(\mathrm{C}), 109.7(\mathrm{C}), 31.4\left(\mathrm{CH}_{2}\right), 21.4\left(\mathrm{CH}_{2}\right), 20.1\left(\mathrm{CH}_{3}\right)$, $13.2\left(\mathrm{CH}_{3}\right) .{ }^{19} \mathbf{F}$ NMR $\left(282 \mathrm{MHz}, \mathrm{CDCl}_{3}, 300 \mathrm{~K}\right): \delta(\mathrm{ppm})=-73.4(\mathrm{~s}, 3 \mathrm{~F}) ;$ HRMS (ESI) $m / z=390.0149$ calcd. for $\mathrm{C}_{14} \mathrm{H}_{13} \mathrm{ClF}_{3} \mathrm{NNaO}_{3} \mathrm{SNa}[\mathrm{M}+\mathrm{Na}]^{+}$, found: 390.0148 ; IR (neat, $\left.\mathrm{cm}^{-1}\right): 2969 w, 2878 w, 2227 w, 1643 w, 1596 w, 1426 s, 1300 w, 1214 s, 1135 s, 1094 w$, $1054 m, 1021 m, 946 w, 894 m, 817 m, 764 w, 690 w$.

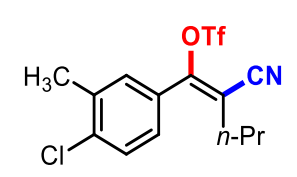

(Z)-1-(4-Bromophenyl)-2-cyanopent-1-en-1-yl trifluoromethane-

sulfonate (2e): The title compound was prepared according to general procedure (GP2) with $\mathrm{Fe}(\mathrm{OAc})_{2}(3.5 \mathrm{mg}, 20 \mu \mathrm{mol}, 10 \mathrm{~mol} \%)$,

L1 (3.6 mg, 20 mol, 10 mol\%), 1-bromo-4-(pent-1-yn-1-yl)benzene 3e (44.4 mg, 
0.200 mmol, 1.0 equiv), and 3,5-di(trifluoromethyl)phenyl(cyano)iodonium triflate 1a (227 mg, $0.440 \mathrm{mmol}, 2.2$ equiv) in DCE ( $1 \mathrm{~mL})$ at $45{ }^{\circ} \mathrm{C}$ for $15 \mathrm{~h}$. Purification via silica gel chromatography (Pentane:EtOAc $=300: 1)$ gave the desired product $\mathbf{2 e}$ as a colorless oil in 90\% yield (71.1 mg). TLC $\mathbf{R}_{\mathrm{f}}=0.35$ (Pentane:EtOAc $\left.=20: 1\right)$; ${ }^{1} \mathbf{H}$ NMR $\left(300 \mathrm{MHz}, \mathrm{CDCl}_{3}, 300 \mathrm{~K}\right): \delta(\mathrm{ppm})=7.58(\mathrm{~d}, J=8.3 \mathrm{~Hz}, 2 \mathrm{H}), 7.24(\mathrm{~d}, J=8.3 \mathrm{~Hz}, 2 \mathrm{H})$, $2.21(\mathrm{t}, J=7.5 \mathrm{~Hz}, 2 \mathrm{H}), 1.61\left(\mathrm{tq}, J^{1}=7.5 \mathrm{~Hz}, J^{2}=7.4 \mathrm{~Hz}, 2 \mathrm{H}\right), 0.86(\mathrm{t}, J=7.4 \mathrm{~Hz}, 3 \mathrm{H})$; ${ }^{13} \mathrm{C}$ NMR $\left(75 \mathrm{MHz}, \mathrm{CDCl}_{3}, 300 \mathrm{~K}\right): \delta(\mathrm{ppm})=154.4(\mathrm{C}), 132.5(\mathrm{CH}), 130.4(\mathrm{CH})$, $128.6(\mathrm{C}), 126.7(\mathrm{C}), 118.1$ (q, $\left.J=321.0 \mathrm{~Hz}, \mathrm{CF}_{3}\right), 114.0(\mathrm{C}), 110.1(\mathrm{C}), 31.4\left(\mathrm{CH}_{2}\right)$, $21.4\left(\mathrm{CH}_{2}\right), 13.1\left(\mathrm{CH}_{3}\right) ;{ }^{19} \mathbf{F}$ NMR $\left(282 \mathrm{MHz}, \mathrm{CDCl}_{3}, 300 \mathrm{~K}\right): \delta(\mathrm{ppm})=-73.4(\mathrm{~s}, 3 \mathrm{~F})$; HRMS (ESI) $m / z=419.9487$ calcd. for $\mathrm{C}_{13} \mathrm{H}_{11} \mathrm{BrF}_{3} \mathrm{NO}_{3} \mathrm{SNa}[\mathrm{M}+\mathrm{Na}]^{+}$, found: 419.9499; IR (neat, $\mathrm{cm}^{-1}$ ): 2968w, 2226w, 1642w, 1588w, 1425s, 1211s, 1133s, 1073m, $996 s, 836 s, 763 w, 685 w$.

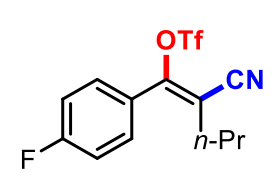

(Z)-2-Cyano-1-(4-fluorophenyl)pent-1-en-1-yl trifluoromethane-

sulfonate (2f): The title compound was prepared according to general procedure (GP2) with $\mathrm{Fe}(\mathrm{OAc})_{2}(3.5 \mathrm{mg}, 20 \mu \mathrm{mol}, 10 \mathrm{~mol} \%)$,

L1 (3.6 mg, 20 mol, 10 mol\%), 1-fluoro-4-(pent-1-yn-1-yl)benzene 3f (32.4 mg, 0.200 mmol, 1.0 equiv), and 3,5-di(trifluoromethyl)phenyl(cyano)iodonium triflate 1a (227 $\mathrm{mg}, 0.440 \mathrm{mmol}, 2.2$ equiv) in DCE $(1 \mathrm{~mL})$ at $45^{\circ} \mathrm{C}$ for $15 \mathrm{~h}$. Purification via silica gel chromatography (Pentane:EtOAc $=300: 1$, then 200:1) gave the desired product $\mathbf{2 f}$ as a colorless oil in 50\% yield (33.7 mg). TLC $\mathbf{R}_{\mathrm{f}}=0.3$ (Pentane:EtOAc $\left.=20: 1\right)$; ${ }^{1} \mathbf{H}$ NMR $\left(300 \mathrm{MHz}, \mathrm{CDCl}_{3}, 300 \mathrm{~K}\right): \delta(\mathrm{ppm})=7.41-7.36(\mathrm{~m}, 2 \mathrm{H}), 7.19-7.10(\mathrm{~m}, 2 \mathrm{H}), 2.21$ $(\mathrm{t}, J=7.5 \mathrm{~Hz}, 2 \mathrm{H}), 1.61\left(\mathrm{tq}, J^{1}=7.5 \mathrm{~Hz}, J^{2}=7.5 \mathrm{~Hz}, 2 \mathrm{H}\right), 0.87(\mathrm{t}, J=7.4 \mathrm{~Hz}, 3 \mathrm{H}) ;{ }^{13} \mathbf{C}$ NMR (75 MHz, $\left.\mathrm{CDCl}_{3}, 300 \mathrm{~K}\right): \delta(\mathrm{ppm})=164.4(\mathrm{~d}, J=254.8 \mathrm{~Hz}, \mathrm{C}), 154.5(\mathrm{C}), 131.2$ $(\mathrm{d}, J=9.0 \mathrm{~Hz}, \mathrm{CH}), 125.9(\mathrm{~d}, J=3.7 \mathrm{~Hz}, \mathrm{C}), 118.1\left(\mathrm{q}, J=321.1 \mathrm{~Hz}, \mathrm{CF}_{3}\right), 116.5(\mathrm{~d}, J$ $=22.3 \mathrm{~Hz}, \mathrm{CH}), 114.1(\mathrm{C}), 109.8(\mathrm{C}), 31.4\left(\mathrm{CH}_{2}\right), 21.4\left(\mathrm{CH}_{2}\right), 13.2\left(\mathrm{CH}_{3}\right) ;{ }^{19} \mathbf{F} \mathbf{~ N M R}$ $\left(282 \mathrm{MHz}, \mathrm{CDCl}_{3}, 300 \mathrm{~K}\right): \delta(\mathrm{ppm})=-73.5(\mathrm{~s}, 3 \mathrm{~F}),-105.7(\mathrm{~s}, 1 \mathrm{~F}) ;$ HRMS (ESI) $\mathrm{m} / \mathrm{z}=$ 360.0288 calcd. for $\mathrm{C}_{13} \mathrm{H}_{11} \mathrm{~F}_{4} \mathrm{NO}_{3} \mathrm{SNa}[\mathrm{M}+\mathrm{Na}]^{+}$, found: 360.0294 ; IR (neat, $\mathrm{cm}^{-1}$ ): $2970 w, 2879 w, 2227 w, 1646 w, 1603 m, 1508 m, 1425 s, 1212 s, 1135 w, 1092 m, 999 s$, $847 s, 813 s, 764 w, 690 w$. 


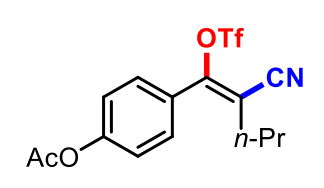

(Z)-4-(2-Cyano-1-(((trifluoromethyl)sulfonyl)oxy)pent-1-en-1-

yl) phenyl acetate (2g): The title compound was prepared according to general procedure (GP2) with $\mathrm{Fe}(\mathrm{OAc})_{2}(3.5 \mathrm{mg}, 20$

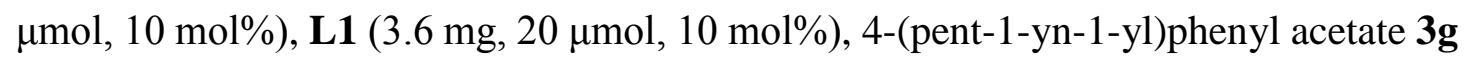
(40.4 mg, $0.200 \mathrm{mmol}, 1.0$ equiv), and 3,5-di(trifluoromethyl)phenyl(cyano)iodonium triflate 1a (227 mg, $0.440 \mathrm{mmol}, 2.2$ equiv) in DCE $(1 \mathrm{~mL})$ at $45{ }^{\circ} \mathrm{C}$ for $15 \mathrm{~h}$. Purification via silica gel chromatography (Pentane:EtOAc $=30: 1$, then 25:1) gave the desired product $2 \mathrm{~g}$ as a colorless oil in $54 \%$ yield (40.6 mg). TLC $\mathbf{R}_{\mathrm{f}}=0.3$ (Pentane:EtOAc = 5:1); ${ }^{1} \mathbf{H}$ NMR $\left(300 \mathrm{MHz}, \mathrm{CDCl}_{3}, 300 \mathrm{~K}\right): \delta(\mathrm{ppm})=7.39(\mathrm{~d}, J=$ $8.6 \mathrm{~Hz}, 2 \mathrm{H}), 7.19(\mathrm{~d}, J=8.6 \mathrm{~Hz}, 2 \mathrm{H}), 2.26(\mathrm{~s}, 3 \mathrm{H}), 2.25(\mathrm{t}, J=8.1 \mathrm{~Hz}, 2 \mathrm{H}) 1.62\left(\mathrm{tq}, J^{1}\right.$ $\left.=7.5 \mathrm{~Hz}, J^{2}=7.2 \mathrm{~Hz}, 2 \mathrm{H}\right), 0.87(\mathrm{t}, J=7.3 \mathrm{~Hz}, 3 \mathrm{H}) ;{ }^{13} \mathbf{C} \mathbf{~ N M R}\left(75 \mathrm{MHz}, \mathrm{CDCl}_{3}, 300\right.$ $\mathrm{K}): \delta(\mathrm{ppm})=168.6(\mathrm{C}), 154.6(\mathrm{C}), 153.1(\mathrm{C}), 130.2(\mathrm{CH}), 127.1(\mathrm{C}), 122.4(\mathrm{CH})$, $118.1\left(\mathrm{q}, J=321.0 \mathrm{~Hz}, \mathrm{CF}_{3}\right), 114.2(\mathrm{C}), 109.8(\mathrm{C}), 31.4\left(\mathrm{CH}_{2}\right), 21.4\left(\mathrm{CH}_{2}\right), 21.1\left(\mathrm{CH}_{3}\right)$, $13.2\left(\mathrm{CH}_{3}\right) ;{ }^{19} \mathbf{F}$ NMR $\left(282 \mathrm{MHz}, \mathrm{CDCl}_{3}, 300 \mathrm{~K}\right): \delta(\mathrm{ppm})=-73.4(\mathrm{~s}, 3 \mathrm{~F}) ;$ HRMS (ESI) $m / z=400.0437$ calcd. for $\mathrm{C}_{15} \mathrm{H}_{14} \mathrm{~F}_{3} \mathrm{NO}_{5} \mathrm{SNa}[\mathrm{M}+\mathrm{Na}]^{+}$, found: $400.0438 ; \mathbf{I R}$ (neat, $\mathrm{cm}^{-}$ 1): 2969w, 2226w, 1772s, 1642w, 1603w, 1505w, 1425s, 1371m, 1195s, 1135m, 1092w, $998 s, 911 m, 852 m, 818 m, 764 w, 679 w$.

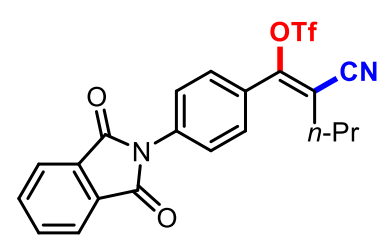

(Z)-2-Cyano-1-(4-(1,3-dioxoisoindolin-2-yl)phenyl)pent1-en-1-yl trifluoromethanesulfonate (2h): The title compound was prepared according to general procedure (GP2) with $\mathrm{Fe}(\mathrm{OAc})_{2}(3.5 \mathrm{mg}, 20 \mu \mathrm{mol}, 10 \mathrm{~mol} \%)$, L1 (3.6 mg, $20 \mu \mathrm{mol}, 10$ mol\%), 2-(4-(pent-1-yn-1-yl)phenyl)isoindoline-1,3-dione 3h (57.8 mg, $0.200 \mathrm{mmol}, 1.0$ equiv), and 3,5-di(trifluoromethyl)phenyl(cyano)iodonium triflate $1 \mathrm{a}$ (227 mg, $0.440 \mathrm{mmol}, 2.2$ equiv) in DCE ( $1 \mathrm{~mL})$ at $45^{\circ} \mathrm{C}$ for $15 \mathrm{~h}$. Purification via silica gel chromatography (Pentane:EtOAc $=20: 1$, the 12:1) gave the desired product $\mathbf{2 h}$ as a colorless oil in $71 \%$ yield $(65.5 \mathrm{mg})$. $\mathbf{T L C} \mathbf{R}_{\mathrm{f}}=0.35$ (Pentane:EtOAc $\left.=4: 1\right) ;{ }^{1} \mathbf{H}$ NMR $\left(300 \mathrm{MHz}, \mathrm{CDCl}_{3}, 300 \mathrm{~K}\right): \delta(\mathrm{ppm})=7.91\left(\mathrm{dd}, J^{1}=5.3 \mathrm{~Hz}, J^{2}=3.1 \mathrm{~Hz}, 2 \mathrm{H}\right)$, $7.76\left(\mathrm{dd}, J^{1}=5.5 \mathrm{~Hz}, J^{2}=3.0 \mathrm{~Hz}, 2 \mathrm{H}\right), 7.64(\mathrm{~d}, J=8.5 \mathrm{~Hz}, 2 \mathrm{H}), 7.51(\mathrm{~d}, J=8.4 \mathrm{~Hz}$, $2 \mathrm{H}), 2.29(\mathrm{t}, J=7.5 \mathrm{~Hz}, 2 \mathrm{H}), 1.64\left(\mathrm{tq}, J^{1}=7.5 \mathrm{~Hz}, J^{2}=7.2 \mathrm{~Hz}, 2 \mathrm{H}\right), 0.89(\mathrm{t}, J=7.3 \mathrm{~Hz}$, 
3H); ${ }^{13} \mathrm{C} \mathrm{NMR}\left(75 \mathrm{MHz}, \mathrm{CDCl}_{3}, 300 \mathrm{~K}\right): \delta(\mathrm{ppm})=166.6(\mathrm{C}), 154.5(\mathrm{C}), 135.1(\mathrm{C})$, $134.8(\mathrm{CH}), 131.4(\mathrm{C}), 129.5(\mathrm{CH}), 128.6(\mathrm{C}), 126.2(\mathrm{CH}), 124.0(\mathrm{CH}), 114.2(\mathrm{C}), 118.2$ $\left(\mathrm{q}, J=319.0 \mathrm{~Hz}, \mathrm{CF}_{3}\right), 110.2(\mathrm{C}), 31.4\left(\mathrm{CH}_{2}\right), 21.4\left(\mathrm{CH}_{2}\right), 13.2\left(\mathrm{CH}_{3}\right) ;{ }^{19}$ F NMR $(282$ $\left.\mathrm{MHz}, \mathrm{CDCl}_{3}, 300 \mathrm{~K}\right): \delta(\mathrm{ppm})=-73.3(\mathrm{~s}, 3 \mathrm{~F})$; HRMS (APCI) $\mathrm{m} / \mathrm{z}=487.0546 \mathrm{calcd}$. for $\mathrm{C}_{21} \mathrm{H}_{15} \mathrm{~F}_{3} \mathrm{~N}_{2} \mathrm{O}_{5} \mathrm{SNa}[\mathrm{M}+\mathrm{Na}]^{+}$, found: 487.0555; IR (neat, $\mathrm{cm}^{-1}$ ): 3074w, 2968w, $2871 w, 2225 w, 1716 s, 1605 w, 1512 m, 1423 s, 1367 s, 1212 s, 1133 s, 1078 s, 998 s, 840 s$.

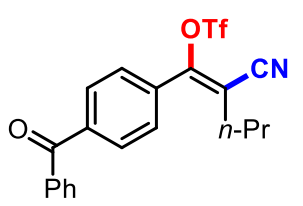

(Z)-1-(4-Benzoylphenyl)-2-cyanopent-1-en-1-yl

trifluoromethanesulfonate (2i): The title compound was prepared according to general procedure (GP2) with $\mathrm{Fe}(\mathrm{OAc})_{2}(3.5 \mathrm{mg}, 20$

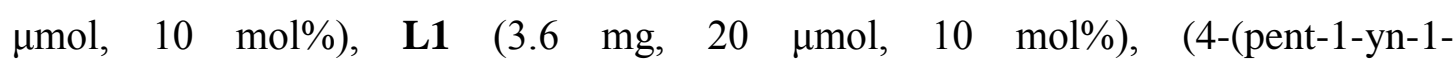
yl)phenyl)(phenyl)methanone $\mathbf{3 i}$ (49.7 mg, $0.200 \mathrm{mmol}, 1.0$ equiv), and 3,5di(trifluoromethyl)phenyl(cyano)iodonium triflate $1 \mathbf{a}(227 \mathrm{mg}, 0.440 \mathrm{mmol}, 2.2$ equiv) in DCE $(1 \mathrm{~mL})$ at $45^{\circ} \mathrm{C}$ for $15 \mathrm{~h}$. Then further portions of $\mathrm{Fe}(\mathrm{OAc})_{2}(3.5 \mathrm{mg}, 20 \mu \mathrm{mol}$, $10 \quad \mathrm{~mol} \%), \quad \mathbf{L 1} \quad(3.6 \quad \mathrm{mg}, \quad 20 \quad \mu \mathrm{mol}, 10 \quad \mathrm{~mol} \%), \quad 3,5-$ di(trifluoromethyl)phenyl(cyano)iodonium triflate 1a (227 mg, $0.440 \mathrm{mmol}, 2.2$ equiv), and DCE $(1 \mathrm{~mL})$ were added. The stirring was continued at $45{ }^{\circ} \mathrm{C}$ for further $15 \mathrm{~h}$. Purification via silica gel chromatography (Pentane:EtOAc $=150: 1$, then 80:1) gave the desired product $2 \mathbf{i}$ as a light yellow oil in $37 \%$ yield $(31.1 \mathrm{mg})$. TLC $\mathbf{R}_{\mathrm{f}}=0.20$ (Pentane:EtOAc = 20:1); ${ }^{1} \mathbf{H}$ NMR $\left(300 \mathrm{MHz}, \mathrm{CDCl}_{3}, 300 \mathrm{~K}\right): \delta(\mathrm{ppm})=7.84(\mathrm{~d}, J=$ $8.5 \mathrm{~Hz}, 2 \mathrm{H}), 7.74-7.71(\mathrm{~m}, 2 \mathrm{H}), 7.59-7.53(\mathrm{~m}, 1 \mathrm{H}), 7.50(\mathrm{~d}, J=8.4 \mathrm{~Hz}, 2 \mathrm{H}), 7.44(\mathrm{t}$, $J=7.5 \mathrm{~Hz}, 2 \mathrm{H}), 2.27(\mathrm{t}, J=7.2 \mathrm{~Hz}, 2 \mathrm{H}), 1.64\left(\mathrm{tq}, J^{1}=7.5 \mathrm{~Hz}, J^{2}=7.5 \mathrm{~Hz} 2 \mathrm{H}\right), 0.88(\mathrm{t}$, $J=7.4 \mathrm{~Hz}, 3 \mathrm{H}) ;{ }^{13} \mathbf{C ~ N M R}\left(75 \mathrm{MHz}, \mathrm{CDCl}_{3}, 300 \mathrm{~K}\right): \delta(\mathrm{ppm})=195.2(\mathrm{C}), 154.3(\mathrm{C})$, $140.3(\mathrm{C}), 136.6(\mathrm{C}), 133.1(\mathrm{CH}), 130.3(\mathrm{CH}), 130.0(\mathrm{CH}), 128.8(\mathrm{CH}), 128.6(\mathrm{CH})$, $118.1\left(\mathrm{q}, J=319.1 \mathrm{~Hz}, \mathrm{CF}_{3}\right), 114.0(\mathrm{C}), 110.8(\mathrm{C}), 31.4\left(\mathrm{CH}_{2}\right), 21.4\left(\mathrm{CH}_{2}\right), 13.2\left(\mathrm{CH}_{3}\right)$; ${ }^{19}$ F NMR $\left(282 \mathrm{MHz}, \mathrm{CDCl}_{3}, 300 \mathrm{~K}\right): \delta(\mathrm{ppm})=-73.3(\mathrm{~s}, 3 \mathrm{~F})$; HRMS (ESI) $\mathrm{m} / \mathrm{z}=$ 446.0644, calcd. for $\mathrm{C}_{20} \mathrm{H}_{16} \mathrm{~F}_{3} \mathrm{NO}_{4} \mathrm{SNa}[\mathrm{M}+\mathrm{Na}]^{+}$, found: 446.0646; IR (neat, $\mathrm{cm}^{-1}$ ): $3065 w, 2969 w, 2877 w, 2226 w, 1662 m, 1600 w, 1426 s, 1276 s, 1212 s, 1134 s, 1092 w$, $1000 s, 924 w, 857 s, 816 s, 701 s, 654 s$. 
<smiles>N#C/C(=C\C(=O)Nc1ccc(OC(F)(F)F)cc1)C(=O)O</smiles>

(Z)-2-Cyano-1-(4-(trifluoromethoxy)phenyl)pent-1-en-1-yl trifluoromethanesulfonate $(\mathbf{2} \mathbf{j})$ : The title compound was prepared according to general procedure (GP2) with $\mathrm{Fe}(\mathrm{OAc})_{2}(3.5 \mathrm{mg}, 20$

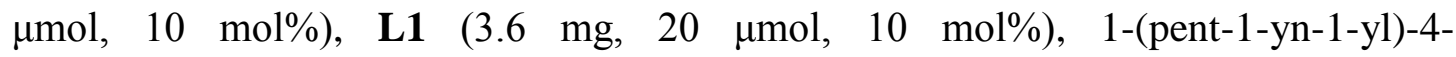
(trifluoromethoxy)benzene $\mathbf{3 j}$ (45.6 $\mathrm{mg}, 0.200 \mathrm{mmol}, 1.0$ equiv), and 3,5di(trifluoromethyl)phenyl(cyano)iodonium triflate $1 \mathbf{a}(227 \mathrm{mg}, 0.440 \mathrm{mmol}, 2.2$ equiv) in DCE $(1 \mathrm{~mL})$ at $45^{\circ} \mathrm{C}$ for $15 \mathrm{~h}$. Purification via silica gel chromatography (Pentane:EtOAc $=300: 1)$ gave the desired product $\mathbf{2} \mathbf{j}$ as a colorless oil in $89 \%$ yield (71.7 mg). TLC $\mathbf{R}_{\mathrm{f}}=0.3$ (Pentane:EtOAc $\left.=20: 1\right) ;{ }^{1} \mathbf{H} \mathbf{~ N M R}\left(300 \mathrm{MHz}, \mathrm{CDCl}_{3}, 300 \mathrm{~K}\right)$ : $\delta(\mathrm{ppm})=7.43(\mathrm{~d}, J=8.6 \mathrm{~Hz}, 2 \mathrm{H}), 7.27(\mathrm{~d}, J=8.5 \mathrm{~Hz}, 2 \mathrm{H}), 2.22(\mathrm{t}, J=7.7 \mathrm{~Hz}, 2 \mathrm{H})$, $1.62\left(\mathrm{tq}, J^{1}=7.5 \mathrm{~Hz}, J^{2}=7.5 \mathrm{~Hz}, 2 \mathrm{H}\right), 0.88(\mathrm{t}, J=7.4 \mathrm{~Hz}, 3 \mathrm{H}) ;{ }^{13} \mathbf{C}$ NMR $(75 \mathrm{MHz}$, $\left.\mathrm{CDCl}_{3}, 300 \mathrm{~K}\right): \delta(\mathrm{ppm})=154.0(\mathrm{C}), 151.5(\mathrm{C}), 130.8(\mathrm{CH}), 128.1(\mathrm{C}), 121.0(\mathrm{CH})$, $120.3\left(\mathrm{q}, J=259.0 \mathrm{~Hz}, \mathrm{OCF}_{3}\right), 118.1\left(\mathrm{q}, J=321.0 \mathrm{~Hz}, \mathrm{CF}_{3}\right), 114.0(\mathrm{C}), 110.5(\mathrm{C}), 31.4$ $\left(\mathrm{CH}_{2}\right), 21.4\left(\mathrm{CH}_{2}\right), 13.2\left(\mathrm{CH}_{3}\right) ;{ }^{19} \mathbf{F} \mathbf{N M R}\left(282 \mathrm{MHz}, \mathrm{CDCl}_{3}, 300 \mathrm{~K}\right): \delta(\mathrm{ppm})=-57.8$ (s, 3F), -73.4 (s, 3F); HRMS (ESI) $m / z=426.0205$ calcd. for $\mathrm{C}_{14} \mathrm{H}_{11} \mathrm{~F}_{6} \mathrm{NO}_{4} \mathrm{SNa}$ $[\mathrm{M}+\mathrm{Na}]^{+}$, found: 426.0211 ; IR (neat, $\mathrm{cm}^{-1}$ ): 2935w, 2227w, 1644w, 1607w, 1427s, $1206 s, 1169 s, 1133 s, 1000 s, 915 w, 853 s, 815 s, 764 m, 659 w$.

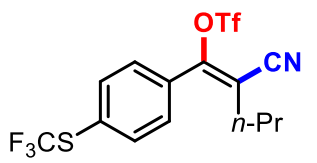

(Z)-2-Cyano-1-(4-((trifluoromethyl)thio)phenyl)pent-1-en-1yl trifluoromethanesulfonate (2k): The title compound was prepared according to general procedure $\left(\right.$ GP2) with $\mathrm{Fe}(\mathrm{OAc})_{2}$ (3.5 mg, $20 \mu \mathrm{mol}, 10 \mathrm{~mol} \%$ ), L1 (3.6 mg, $20 \mu \mathrm{mol}, 10 \mathrm{~mol} \%$ ), (4-(pent-1-yn-1yl)phenyl)(trifluoromethyl)sulfane $3 \mathbf{k}(48.9 \mathrm{mg}, 0.200 \mathrm{mmol}, 1.0$ equiv), and 3,5di(trifluoromethyl)phenyl(cyano)iodonium triflate 1a $(227 \mathrm{mg}, 0.440 \mathrm{mmol}, 2.2$ equiv) in DCE $(1 \mathrm{~mL})$ at $45^{\circ} \mathrm{C}$ for $15 \mathrm{~h}$. Then further portions of $\mathrm{Fe}(\mathrm{OAc})_{2}(3.5 \mathrm{mg}, 20 \mu \mathrm{mol}$, $10 \mathrm{~mol} \%), \quad \mathbf{L 1} \quad(3.6 \quad \mathrm{mg}, \quad 20 \quad \mu \mathrm{mol}, 10 \quad \mathrm{~mol} \%), \quad 3,5-$ di(trifluoromethyl)phenyl(cyano)iodonium triflate 1a (227 mg, $0.440 \mathrm{mmol}, 2.2$ equiv), and DCE $(1 \mathrm{~mL})$ were added. The stirring was continued at $45{ }^{\circ} \mathrm{C}$ for further $15 \mathrm{~h}$. Purification via silica gel chromatography (Pentane:EtOAc $=250: 1)$ gave the desired product $2 \mathbf{k}$ as a light yellow oil in $61 \%$ yield $\left(51.2 \mathrm{mg}\right.$ ). $\mathbf{T L C} \mathbf{R}_{\mathrm{f}}=0.25$ (Pentane:EtOAc 
$=40: 1) ;{ }^{1} \mathbf{H}$ NMR $\left(300 \mathrm{MHz}, \mathrm{CDCl}_{3}, 300 \mathrm{~K}\right): \delta(\mathrm{ppm})=7.71(\mathrm{~d}, J=8.3 \mathrm{~Hz}, 2 \mathrm{H}), 7.43$ $(\mathrm{d}, J=8.5 \mathrm{~Hz}, 2 \mathrm{H}), 2.24(\mathrm{t}, J=7.7 \mathrm{~Hz}, 2 \mathrm{H}), 1.64\left(\mathrm{tq}, J^{1}=7.5 \mathrm{~Hz}, J^{2}=7.5 \mathrm{~Hz}, 2 \mathrm{H}\right)$, $0.88(\mathrm{t}, J=7.4 \mathrm{~Hz}, 3 \mathrm{H}) ;{ }^{13} \mathbf{C}$ NMR $\left(75 \mathrm{MHz}, \mathrm{CDCl}_{3}, 300 \mathrm{~K}\right): \delta(\mathrm{ppm})=153.9(\mathrm{C})$, $136.0(\mathrm{CH}), 132.0(\mathrm{C}), 129.7(\mathrm{CH}), 129.2(\mathrm{C}), 129.2$ (q, $\left.J=308.6 \mathrm{~Hz}, \mathrm{SCF}_{3}\right), 118.1$ (q, $\left.J=321.0 \mathrm{~Hz}, \mathrm{CF}_{3}\right), 113.9(\mathrm{C}), 111.1(\mathrm{C}), 31.4\left(\mathrm{CH}_{2}\right), 21.4\left(\mathrm{CH}_{2}\right), 13.2\left(\mathrm{CH}_{3}\right) ;{ }^{19} \mathbf{F}$ NMR $\left(282 \mathrm{MHz}, \mathrm{CDCl}_{3}, 300 \mathrm{~K}\right): \delta(\mathrm{ppm})=-41.7(\mathrm{~s}, 3 \mathrm{~F}),-73.3(\mathrm{~s}, 3 \mathrm{~F}) ;$ HRMS (ESI) $\mathrm{m} / \mathrm{z}=$ 441.9977 calcd. for $\mathrm{C}_{14} \mathrm{H}_{11} \mathrm{~F}_{6} \mathrm{NO}_{3} \mathrm{~S}_{2} \mathrm{Na}[\mathrm{M}+\mathrm{Na}]^{+}$, found: 441.9971; IR (neat, $\mathrm{cm}^{-1}$ ): $2970 w, 2228 w, 1644 s, 1427 s, 1215 s, 1113 s, 1000 s, 843 s, 811 s, 758 m, 688 w$.

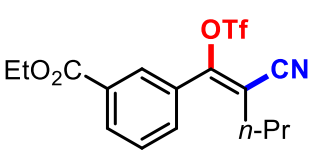

(Z)-2-Cyano-1-(4-((trifluoromethyl)thio)phenyl)pent-1-en-1yl trifluoromethanesulfonate (21): The title compound was prepared according to general procedure (GP2) with $\mathrm{Fe}(\mathrm{OAc})_{2}$ (3.5 mg, $20 \mu \mathrm{mol}, 10 \mathrm{~mol} \%$ ), L1 (3.6 mg, $20 \mu \mathrm{mol}, 10 \mathrm{~mol} \%)$, 1-(pent-1-yn-1-yl)- ethyl 3-(pent-1-yn-1-yl)benzoate 31 (43.3 mg, $0.200 \mathrm{mmol}, 1.0$ equiv), and 3,5di(trifluoromethyl)phenyl(cyano)iodonium triflate 1a (227 mg, $0.440 \mathrm{mmol}, 2.2$ equiv) in DCE $(1 \mathrm{~mL})$ at $45^{\circ} \mathrm{C}$ for $15 \mathrm{~h}$. Purification via silica gel chromatography (Pentane:EtOAc $=100: 1$, then 60:1) gave the desired product $\mathbf{2 l}$ as a colorless oil in $48 \%$ yield (37.6 mg). $\mathbf{T L C} \mathbf{R}_{\mathrm{f}}=0.4$ (Pentane:EtOAc $\left.=10: 1\right) ;{ }^{1} \mathbf{H}$ NMR $\left(300 \mathrm{MHz}, \mathrm{CDCl}_{3}\right.$, $300 \mathrm{~K}): \delta(\mathrm{ppm})=8.15(\mathrm{~d}, J=6.9 \mathrm{~Hz}, 1 \mathrm{H}), 8.05(\mathrm{~s}, 1 \mathrm{H}), 7.64-7.51(\mathrm{~m}, 2 \mathrm{H}), 4.35(\mathrm{q}$, $J=7.1 \mathrm{~Hz}, 2 \mathrm{H}), 2.24(\mathrm{t}, J=7.5 \mathrm{~Hz}, 2 \mathrm{H}), 1.64\left(\mathrm{tq}, J^{1}=7.5 \mathrm{~Hz}, J^{2}=7.2 \mathrm{~Hz}, 2 \mathrm{H}\right), 1.35$ $(\mathrm{t}, J=7.1 \mathrm{~Hz}, 3 \mathrm{H}), 0.88(\mathrm{t}, J=7.4 \mathrm{~Hz}, 3 \mathrm{H}) ;{ }^{13} \mathbf{C} \mathbf{N M R}\left(75 \mathrm{MHz}, \mathrm{CDCl}_{3}, 300 \mathrm{~K}\right): \delta$ $(\mathrm{ppm})=165.0(\mathrm{C}), 154.4(\mathrm{C}), 132.7(\mathrm{CH}), 132.6(\mathrm{CH}), 131.6(\mathrm{C}), 130.1(\mathrm{C}), 129.9$ (CH), $129.3(\mathrm{CH}), 118.1$ (q, $\left.J=320.9 \mathrm{~Hz}, \mathrm{CF}_{3}\right), 114.1(\mathrm{C}), 110.5(\mathrm{C}), 61.7\left(\mathrm{CH}_{2}\right), 31.4$ $\left(\mathrm{CH}_{2}\right), 21.4\left(\mathrm{CH}_{2}\right), 14.2\left(\mathrm{CH}_{3}\right), 13.2\left(\mathrm{CH}_{3}\right) ;{ }^{19} \mathbf{F}$ NMR $\left(282 \mathrm{MHz}, \mathrm{CDCl}_{3}, 300 \mathrm{~K}\right): \delta$ $(\mathrm{ppm})=-73.3(\mathrm{~s}, 3 \mathrm{~F}) ;$ HRMS $(\mathrm{ESI}) \mathrm{m} / \mathrm{z}=414.0593$ calcd. for $\mathrm{C}_{16} \mathrm{H}_{16} \mathrm{~F}_{3} \mathrm{NO}_{5} \mathrm{SNa}$ $[\mathrm{M}+\mathrm{Na}]^{+}$, found: 414.0599; IR (neat, $\mathrm{cm}^{-1}$ ): 2924w, 2227w, 1723s, 1645w, 1427s, $1370 w, 1302 m, 1221 s, 1135 s, 1012 m, 894 w, 824 m, 763 w, 727 w, 684 w$.<smiles>N#C/C(=C\C(=O)NC(=O)c1cccc(C=O)c1)C(=O)O</smiles>

(Z)-2-Cyano-1-(3-formylphenyl)pent-1-en-1-yl

methanesulfonate (2m): The title compound was prepared 
according to general procedure (GP2) with $\mathrm{Fe}(\mathrm{OAc})_{2}(3.5 \mathrm{mg}, 20 \mu \mathrm{mol}, 10 \mathrm{~mol} \%), \mathbf{L 1}$ (3.6 mg, $20 \mu \mathrm{mol}, 10$ mol\%), 3-(pent-1-yn-1-yl)benzaldehyde 3m (34.5 mg, 0.200 mmol, 1.0 equiv), and 3,5-di(trifluoromethyl)phenyl(cyano)iodonium triflate 1a (227 $\mathrm{mg}, 0.440 \mathrm{mmol}, 2.2$ equiv) in DCE $(1 \mathrm{~mL})$ at $45^{\circ} \mathrm{C}$ for $15 \mathrm{~h}$. Purification via silica gel chromatography (Pentane:EtOAc $=35: 1$, then 20:1) gave the desired product $\mathbf{2} \mathbf{m}$ as a light yellow oil in $13 \%$ yield $(9.0 \mathrm{mg})$. $\mathbf{T L C} \mathbf{R}_{\mathrm{f}}=0.25$ (Pentane:EtOAc $\left.=7: 1\right) ;{ }^{1} \mathbf{H}$ NMR $\left(300 \mathrm{MHz}, \mathrm{CDCl}_{3}, 300 \mathrm{~K}\right): \delta(\mathrm{ppm})=10.00(\mathrm{~s}, 1 \mathrm{H}), 8.01-7.98(\mathrm{~m}, 1 \mathrm{H}), 7.88(\mathrm{~s}, 1 \mathrm{H})$, $7.65-7.64(\mathrm{~m}, 2 \mathrm{H}), 2.24(\mathrm{t}, J=7.9 \mathrm{~Hz}, 2 \mathrm{H}), 1.64\left(\mathrm{tq}, J^{1}=7.5 \mathrm{~Hz}, J^{2}=7.4 \mathrm{~Hz}, 2 \mathrm{H}\right)$, $0.88(\mathrm{t}, J=7.4 \mathrm{~Hz}, 3 \mathrm{H}) ;{ }^{13} \mathbf{C} \mathbf{~ N M R}\left(75 \mathrm{MHz}, \mathrm{CDCl}_{3}, 300 \mathrm{~K}\right): \delta(\mathrm{ppm})=190.4(\mathrm{CH})$, $153.9(\mathrm{C}), 136.9(\mathrm{C}), 134.1(\mathrm{CH}), 132.7(\mathrm{CH}), 131.0(\mathrm{C}), 130.0(\mathrm{CH}), 129.6(\mathrm{CH}), 118.1$ (q, $\left.J=321.1 \mathrm{~Hz}, \mathrm{CF}_{3}\right), 113.9(\mathrm{C}), 110.9(\mathrm{C}), 31.5\left(\mathrm{CH}_{2}\right), 21.4\left(\mathrm{CH}_{2}\right), 13.2\left(\mathrm{CH}_{3}\right) ;{ }^{19} \mathbf{F}$ NMR (282 MHz, $\left.\mathrm{CDCl}_{3}, 300 \mathrm{~K}\right): \delta(\mathrm{ppm})=-73.3(\mathrm{~s}, 3 \mathrm{~F})$; HRMS (ESI) $m / z=370.0331$ calcd. for $\mathrm{C}_{14} \mathrm{H}_{12} \mathrm{~F}_{3} \mathrm{NO}_{4} \mathrm{SNa}[\mathrm{M}+\mathrm{Na}]^{+}$, found: 370.0339; IR (neat, $\mathrm{cm}^{-1}$ ): 3064w, 2930w, $2853 w, 2735 w, 2359 w, 2227 w, 1705 s, 1649 w, 1601 w, 1426 s, 1213 s, 1134 s, 1092 w$, $1016 m, 951 w, 895 w, 818 s, 763 w$.

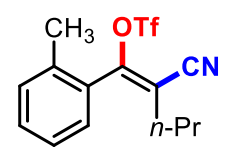

(Z)-2-Cyano-1-(o-tolyl)pent-1-en-1-yl trifluoromethanesulfonate

(2n): The title compound was prepared according to general procedure (GP2) with $\mathrm{Fe}(\mathrm{OAc})_{2}(3.5 \mathrm{mg}, 20 \mu \mathrm{mol}, 10 \mathrm{~mol} \%)$, L1 (3.6 mg, 20 $\mu \mathrm{mol}, 10$ mol\%), 1-methyl-2-(pent-1-yn-1-yl)benzene 3n (31.6 mg, 0.200 mmol, 1.0 equiv), and 3,5-di(trifluoromethyl)phenyl(cyano)iodonium triflate 1a (227 mg, 0.440 mmol, 2.2 equiv) in DCE $(1 \mathrm{~mL})$ at $45^{\circ} \mathrm{C}$ for $15 \mathrm{~h}$. Purification via silica gel chromatography (Pentane:EtOAc $=300: 1)$ gave the desired product $\mathbf{2 n}$ as a colorless oil in 30\% yield (20.0 mg). TLC $\mathbf{R}_{\mathrm{f}}=0.45$ (Pentane:EtOAc $\left.=20: 1\right)$; ${ }^{1} \mathbf{H}$ NMR (300 $\left.\mathrm{MHz}, \mathrm{CDCl}_{3}, 300 \mathrm{~K}\right): \delta(\mathrm{ppm})=7.39-7.32(\mathrm{~m}, 1 \mathrm{H}), 7.25-7.19(\mathrm{~m}, 3 \mathrm{H}), 2.28(\mathrm{~s}, 3 \mathrm{H})$, $2.05(\mathrm{q}, J=7.5 \mathrm{~Hz}, 2 \mathrm{H}), 1.57\left(\mathrm{tq}, J^{1}=7.5 \mathrm{~Hz}, J^{2}=7.4 \mathrm{~Hz}, 2 \mathrm{H}\right), 0.81(\mathrm{t}, J=7.4 \mathrm{~Hz}, 3 \mathrm{H})$; ${ }^{13} \mathrm{C} \mathrm{NMR}\left(75 \mathrm{MHz}, \mathrm{CDCl}_{3}, 300 \mathrm{~K}\right): \delta(\mathrm{ppm})=155.8(\mathrm{C}), 137.7(\mathrm{C}), 131.8(\mathrm{CH}), 131.0$ $(\mathrm{CH}), 130.2(\mathrm{CH}), 128.6(\mathrm{C}), 126.1(\mathrm{CH}), 118.1\left(\mathrm{q}, J=320.9 \mathrm{~Hz}, \mathrm{CF}_{3}\right), 114.1(\mathrm{C}), 110.7$ (C), $31.4\left(\mathrm{CH}_{2}\right), 21.0\left(\mathrm{CH}_{2}\right), 19.4\left(\mathrm{CH}_{3}\right), 13.1\left(\mathrm{CH}_{3}\right) ;{ }^{19} \mathbf{F} \mathbf{~ N M R}\left(282 \mathrm{MHz}, \mathrm{CDCl}_{3}, 300\right.$ $\mathrm{K}): \delta(\mathrm{ppm})=-74.0(\mathrm{~s}, 3 \mathrm{~F}) ;$ HRMS (ESI) $m / z=356.0539$, calcd. for $\mathrm{C}_{14} \mathrm{H}_{14} \mathrm{~F}_{3} \mathrm{NO}_{3} \mathrm{SNa}$ 
$[\mathrm{M}+\mathrm{Na}]^{+}$, found: 356.0541 ; IR (neat, $\mathrm{cm}^{-1}$ ): 2968w, 2834w, 2257w, 2228w, 1649w, $1425 m, 1214 s, 1135 s, 1091 m, 990 m, 908 s, 867 w, 820 s, 768 w, 728 s, 649 w$.

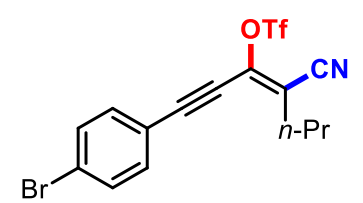

(Z)-1-(4-Bromophenyl)-4-cyanohept-3-en-1-yn-3-yl trifluoromethanesulfonate (2o): The title compound was prepared according to general procedure $\left(\right.$ GP2) with $\mathrm{Fe}(\mathrm{OAc})_{2}$ (3.5 mg, $20 \mu \mathrm{mol}, 10 \mathrm{~mol} \%$ ), L1 (3.6 mg, $20 \mu \mathrm{mol}, 10 \mathrm{~mol} \%$ ), 1-bromo-4-(hepta-1,3diyn-1-yl)benzene $\quad 30 \quad(49.4 \quad \mathrm{mg}, \quad 0.200 \mathrm{mmol}, \quad 1.0$ equiv), and 3,5di(trifluoromethyl)phenyl(cyano)iodonium triflate 1a $(227 \mathrm{mg}, 0.440 \mathrm{mmol}, 2.2$ equiv) in DCE $(1 \mathrm{~mL})$ at $45^{\circ} \mathrm{C}$ for $15 \mathrm{~h}$. Purification via silica gel chromatography (Pentane:EtOAc $=400: 1$, then 300:1) gave the desired product 20 as a yellow oil in $61 \%$ yield (51.0 mg). TLC $\mathbf{R}_{\mathrm{f}}=0.5$ (Pentane:EtOAc $\left.=20: 1\right)$; ${ }^{1} \mathbf{H} \mathbf{~ N M R}\left(300 \mathrm{MHz}, \mathrm{CDCl}_{3}\right.$, $300 \mathrm{~K}): \delta(\mathrm{ppm})=7.49(\mathrm{~d}, J=8.6 \mathrm{~Hz}, 2 \mathrm{H}), 7.30(\mathrm{~d}, J=8.6 \mathrm{~Hz}, 2 \mathrm{H}), 2.43(\mathrm{t}, J=7.3 \mathrm{~Hz}$, 2H), 1.67 (tq, $\left.J^{1}=7.5 \mathrm{~Hz}, J^{2}=7.5 \mathrm{~Hz}, 2 \mathrm{H}\right), 0.96(\mathrm{t}, J=7.4 \mathrm{~Hz}, 3 \mathrm{H}) ;{ }^{13} \mathbf{C}$ NMR $(75$ $\left.\mathrm{MHz}, \mathrm{CDCl}_{3}, 300 \mathrm{~K}\right): \delta(\mathrm{ppm})=137.3(\mathrm{C}), 133.3(\mathrm{CH}), 132.3(\mathrm{CH}), 126.0(\mathrm{C}), 118.4$ (q, $\left.J=319.1 \mathrm{~Hz}, \mathrm{CF}_{3}\right), 118.3(\mathrm{C}), 114.8(\mathrm{C}), 113.7(\mathrm{C}), 104.4(\mathrm{C}), 79.3(\mathrm{C}), 32.6\left(\mathrm{CH}_{2}\right)$, $21.0\left(\mathrm{CH}_{2}\right), 13.1\left(\mathrm{CH}_{3}\right) ;{ }^{19} \mathbf{F}$ NMR $\left(282 \mathrm{MHz}, \mathrm{CDCl}_{3}, 300 \mathrm{~K}\right): \delta(\mathrm{ppm})=-72.9(\mathrm{~s}, 3 \mathrm{~F})$; HRMS (ESI) $m / z=445.9487$, calcd. for $\mathrm{C}_{15} \mathrm{H}_{11} \mathrm{BrF}_{3} \mathrm{NO}_{3} \mathrm{SNa}[\mathrm{M}+\mathrm{Na}]^{+}$, found: 445.9481; IR (neat, $\mathrm{cm}^{-1}$ ): 2968w, 2877w, 2200w, 1618w, 1582w, 1486w, 1430s, $1306 w, 1212 s, 1134 s, 1083 s, 1011 m, 969 s, 889 w, 817 s, 760 w, 703 w$.

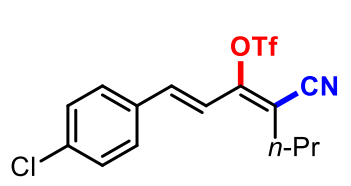

(1E, 3Z)-1-(4-Chlorophenyl)-4-cyanohepta-1,3-dien-3-yl trifluoromethanesulfonate (2p): The title compound was prepared according to general procedure (GP2) with $\mathrm{Fe}(\mathrm{OAc})_{2}$ (3.5 mg, 20 mol, $10 \mathrm{~mol} \%$ ), L1 (3.6 mg, 20 mol, 10 mol\%), (E)-1-chloro-4-(hept-1en-3-yn-1-yl)benzene $\quad 3 p \quad(40.8 \mathrm{mg}, \quad 0.200 \mathrm{mmol}, \quad 1.0$ equiv), and 3,5di(trifluoromethyl)phenyl(cyano)iodonium triflate 1a (227 mg, $0.440 \mathrm{mmol}, 2.2$ equiv) in DCE $(1 \mathrm{~mL})$ at $45^{\circ} \mathrm{C}$ for $15 \mathrm{~h}$. Purification via silica gel chromatography $($ Pentane:EtOAc $=300: 1) \quad$ gave the desired product $\mathbf{2 p}$ as a yellow oil in $20 \%$ yield (15.2 mg). TLC $\mathbf{R}_{\mathrm{f}}=0.35$ (Pentane:EtOAc $\left.=20: 1\right) ;{ }^{1} \mathbf{H}$ NMR $\left(300 \mathrm{MHz}, \mathrm{CDCl}_{3}, 300\right.$ 
$\mathrm{K}): \delta(\mathrm{ppm})=7.36(\mathrm{~d}, J=8.6 \mathrm{~Hz}, 2 \mathrm{H}), 7.30(\mathrm{~d}, J=8.7 \mathrm{~Hz}, 2 \mathrm{H}), 7.11(\mathrm{~d}, J=15.8 \mathrm{~Hz}$, $1 \mathrm{H}), 6.78(\mathrm{~d}, J=15.8 \mathrm{~Hz}, 1 \mathrm{H}), 2.38(\mathrm{t}, J=7.4 \mathrm{~Hz}, 2 \mathrm{H}), 1.64\left(\mathrm{tq}, J^{1}=7.5 \mathrm{~Hz}, J^{2}=7.2\right.$ $\mathrm{Hz}, 2 \mathrm{H}), 0.96(\mathrm{t}, J=7.4 \mathrm{~Hz}, 3 \mathrm{H}) ;{ }^{13} \mathbf{C} \mathbf{N M R}\left(75 \mathrm{MHz}, \mathrm{CDCl}_{3}, 300 \mathrm{~K}\right): \delta(\mathrm{ppm})=152.9$ (C), $137.9(\mathrm{CH}), 136.6(\mathrm{C}), 132.8(\mathrm{C}), 129.4(\mathrm{CH}), 129.0(\mathrm{CH}), 116.1(\mathrm{CH}), 118.3$ (q, $\left.J=320.8 \mathrm{~Hz}, \mathrm{CF}_{3}\right), 115.0(\mathrm{C}), 108.0(\mathrm{C}), 31.3\left(\mathrm{CH}_{2}\right), 21.5\left(\mathrm{CH}_{2}\right), 13.2\left(\mathrm{CH}_{3}\right) ;{ }^{19} \mathbf{F ~ N M R}$ $\left(282 \mathrm{MHz}, \mathrm{CDCl}_{3}, 300 \mathrm{~K}\right): \delta(\mathrm{ppm})=-72.5(\mathrm{~s}, 3 \mathrm{~F})$; HRMS (ESI) $\mathrm{m} / \mathrm{z}=402.0149$, calcd. for $\mathrm{C}_{15} \mathrm{H}_{13} \mathrm{ClF}_{3} \mathrm{NO}_{3} \mathrm{SNa}[\mathrm{M}+\mathrm{Na}]^{+}$, found: 402.0161; IR (neat, $\mathrm{cm}^{-1}$ ): 2968w, 2877w, $2217 w, 1624 m, 1587 w, 1491 w, 1410 s, 1331 w, 1209 s, 1133 s, 1091 s, 958 s, 894 m, 807 s$, $743 w, 655 w$.

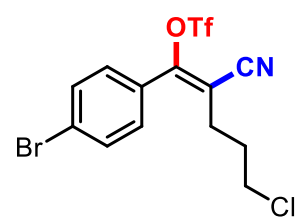

(Z)-1-(4-Bromophenyl)-5-chloro-2-cyanopent-1-en-1-yl tri-

fluoromethanesulfonate $\mathbf{( 2 q )}$ : The title compound was prepared according to general procedure (GP2) with $\mathrm{Fe}(\mathrm{OAc})_{2}(3.5 \mathrm{mg}, 20$ $\mu \mathrm{mol}, 10 \mathrm{~mol} \%$ ), L1 (3.6 mg, 20 mol, 10 mol\%), 1-bromo-4-(5-

chloropent-1-yn-1-yl)benzene $\mathbf{3 q}$ (51.5 mg, $0.200 \mathrm{mmol}, 1.0$ equiv), and 3,5di(trifluoromethyl)phenyl(cyano)iodonium triflate $1 \mathbf{a}(227 \mathrm{mg}, 0.440 \mathrm{mmol}, 2.2$ equiv) in DCE $(1 \mathrm{~mL})$ at $45{ }^{\circ} \mathrm{C}$ for $15 \mathrm{~h}$. Purification via silica gel chromatography (Pentane:EtOAc = 200:1, then 100:1) gave the desired product 2q as a yellow oil in $72 \%$ yield (61.8 mg). TLC $\mathbf{R}_{\mathrm{f}}=0.2$ (Pentane:EtOAc $\left.=20: 1\right) ;{ }^{1} \mathbf{H} \mathbf{~ N M R}\left(300 \mathrm{MHz}, \mathrm{CDCl}_{3}\right.$, $300 \mathrm{~K}): \delta(\mathrm{ppm})=7.60(\mathrm{~d}, J=8.5 \mathrm{~Hz}, 2 \mathrm{H}), 7.27(\mathrm{~d}, J=8.5 \mathrm{~Hz}, 2 \mathrm{H}), 3.48(\mathrm{t}, J=6.0 \mathrm{~Hz}$, 2H), $2.49-2.44(\mathrm{~m}, 2 \mathrm{H}), 2.10-2.01(\mathrm{~m}, 2 \mathrm{H}) ;{ }^{13} \mathrm{C} \mathrm{NMR}\left(75 \mathrm{MHz}, \mathrm{CDCl}_{3}, 300 \mathrm{~K}\right): \delta$ $(\mathrm{ppm})=155.3(\mathrm{C}), 132.6(\mathrm{CH}), 130.2(\mathrm{CH}), 128.3(\mathrm{C}), 127.0(\mathrm{C}), 118.1(\mathrm{q}, J=319.1$ $\left.\mathrm{Hz}, \mathrm{CF}_{3}\right), 113.8(\mathrm{C}), 108.4(\mathrm{C}), 43.0\left(\mathrm{CH}_{2}\right), 30.3\left(\mathrm{CH}_{2}\right), 26.9\left(\mathrm{CH}_{2}\right) ;{ }^{19}$ F NMR (282 $\left.\mathrm{MHz}, \mathrm{CDCl}_{3}, 300 \mathrm{~K}\right): \delta(\mathrm{ppm})=-73.3(\mathrm{~s}, 3 \mathrm{~F})$; HRMS (ESI) $\mathrm{m} / \mathrm{z}=455.9098$ calcd. for $\mathrm{C}_{13} \mathrm{H}_{10} \mathrm{BrClF}_{3} \mathrm{NO}_{3} \mathrm{SNa}[\mathrm{M}+\mathrm{Na}]^{+}$, found:455.9093; IR (neat, $\mathrm{cm}^{-1}$ ): 2970m, 2933w, $1872 w, 2230 w, 1899 w, 1646 w, 1485 s, 1393 s, 1322 s, 1256 w, 1157 w, 1071 s, 1011 s$, $942 m, 822 s, 708 w$. 
<smiles>N#C/C(CC[SeH])=C(\O)c1ccc(Br)cc1</smiles>
(Z)-4-(4-Bromophenyl)-3-cyano-4-(((trifluoromethyl)sulfonyl) -oxy)but-3-en-1-yl 4-methylbenzenesulfonate (2r): The title compound was prepared according to general procedure (GP2) with $\mathrm{Fe}(\mathrm{OAc})_{2}(3.5 \mathrm{mg}, 20 \mu \mathrm{mol}, 10 \mathrm{~mol} \%)$, L1 (3.6 mg, $\left.20 \mu \mathrm{mol}, 10 \mathrm{~mol} \%\right)$, 4-(4bromophenyl)but-3-yn-1-yl 4-methylbenzenesulfonate 3r (75.9 mg, $0.200 \mathrm{mmol}, 1.0$ equiv), and 3,5-di(trifluoromethyl)phenyl(cyano)iodonium triflate 1a (227 mg, 0.440 mmol, 2.2 equiv) in DCE $(1 \mathrm{~mL})$ at $45^{\circ} \mathrm{C}$ for $15 \mathrm{~h}$. Then further portions of $\mathrm{Fe}(\mathrm{OAc})_{2}$ (3.5 mg, $20 \mu \mathrm{mol}, 10 \mathrm{~mol} \%), \quad \mathbf{L}$ (3.6 mg, $20 \mu \mathrm{mol}, 10 \mathrm{~mol} \%), 3,5-$ di(trifluoromethyl)phenyl(cyano)iodonium triflate 1a (227 mg, $0.440 \mathrm{mmol}, 2.2$ equiv), and DCE $(1 \mathrm{~mL})$ were added. The stirring was continued at $45 \mathrm{oC}$ for further $15 \mathrm{~h}$. Purification via silica gel chromatography (Pentane:EtOAc $=30: 1$, then 10:1) gave the desired product $2 \mathbf{r}$ as a yellow oil in $46 \%$ yield $(51.1 \mathrm{mg})$. TLC $\mathbf{R}_{\mathrm{f}}=0.25$ (Pentane:EtOAc = 7:1); ${ }^{1} \mathbf{H}$ NMR $\left(300 \mathrm{MHz}, \mathrm{CDCl}_{3}, 300 \mathrm{~K}\right): \delta(\mathrm{ppm})=7.69(\mathrm{~d}, J=$ $8.3 \mathrm{~Hz}, 2 \mathrm{H}), 7.58(\mathrm{~d}, J=8.4 \mathrm{~Hz}, 2 \mathrm{H}), 7.30(\mathrm{~d}, J=8.2 \mathrm{~Hz}, 2 \mathrm{H}), 7.26(\mathrm{~d}, J=8.6 \mathrm{~Hz}, 2 \mathrm{H})$, $4.15(\mathrm{t}, J=5.9 \mathrm{~Hz}, 2 \mathrm{H}), 2.58(\mathrm{t}, J=5.9 \mathrm{~Hz}, 2 \mathrm{H}), 2.39(\mathrm{~s}, 3 \mathrm{H}) ;{ }^{13} \mathbf{C}$ NMR $(75 \mathrm{MHz}$, $\left.\mathrm{CDCl}_{3}, 300 \mathrm{~K}\right): \delta(\mathrm{ppm})=157.0(\mathrm{C}), 146.0(\mathrm{C}), 132.6(\mathrm{CH}), 132.1(\mathrm{C}), 130.4(\mathrm{CH})$, $130.1(\mathrm{CH}), 127.9(\mathrm{CH}), 127.7(\mathrm{C}), 127.3(\mathrm{C}), 118.0$ (q, J = 321.1 Hz, $\left.\mathrm{CF}_{3}\right), 113.1(\mathrm{C})$, $105.0(\mathrm{C}), 65.5\left(\mathrm{CH}_{2}\right), 29.7\left(\mathrm{CH}_{2}\right), 21.6\left(\mathrm{CH}_{3}\right) ;{ }^{19} \mathbf{F ~ N M R}\left(282 \mathrm{MHz}, \mathrm{CDCl}_{3}, 300 \mathrm{~K}\right): \delta$ $(\mathrm{ppm})=-73.2(\mathrm{~s}, 3 \mathrm{~F})$; HRMS (ESI) $m / z=577.9368$, calcd. for $\mathrm{C}_{19} \mathrm{H}_{15} \mathrm{BrF}_{3} \mathrm{NO}_{6} \mathrm{~S}_{2} \mathrm{Na}$ $[\mathrm{M}+\mathrm{Na}]^{+}$, found: 577.9389; IR (neat, $\mathrm{cm}^{-1}$ ): 2963w, 2228w, 1644w, 1589w, 1486w, $1426 s, 1364 m, 1216 s, 1176 s, 1134 s, 1072 m, 991 s, 906 m, 838 s, 807 s, 763 s, 663 m, 838 s$.

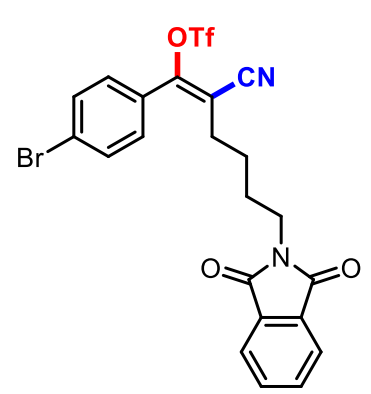

(Z)-1-(4-Bromophenyl)-2-cyano-6-(1,3-dioxoisoindolin-2yl)hex-1-en-1-yl trifluoromethanesulfonate (2s): The title compound was prepared according to general procedure (GP2) with $\mathrm{Fe}(\mathrm{OAc})_{2}(3.5 \mathrm{mg}, 20 \mu \mathrm{mol}, 10 \mathrm{~mol} \%)$, L1 $(3.6$

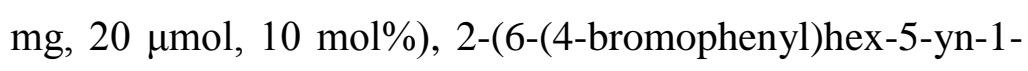
yl)isoindoline-1,3-dione $3 \mathrm{~s}$ (76.5 mg, $0.200 \mathrm{mmol}, 1.0$ equiv), and 3,5-di(trifluoromethyl)phenyl(cyano)iodonium triflate $1 \mathbf{a}(227 \mathrm{mg}, 0.440 \mathrm{mmol}$, 2.2 equiv) in DCE ( $1 \mathrm{~mL})$ at $45{ }^{\circ} \mathrm{C}$ for $15 \mathrm{~h}$. Purification via silica gel chromatography 
(Pentane:EtOAc $=30: 1$, then 14:1) gave the desired product $2 \mathrm{~s}$ as a light yellow oil in 93\% yield (103.9 mg). TLC $\mathbf{R}_{\mathrm{f}}=0.25$ (Pentane:EtOAc $\left.=7: 1\right) ;{ }^{1} \mathbf{H}$ NMR $(300 \mathrm{MHz}$, $\left.\mathrm{CDCl}_{3}, 300 \mathrm{~K}\right): \delta(\mathrm{ppm})=7.79-7.73(\mathrm{~m}, 2 \mathrm{H}), 7.67-7.63(\mathrm{~m}, 2 \mathrm{H}), 7.57(\mathrm{~d}, J=8.5$ $\mathrm{Hz}, 2 \mathrm{H}), 7.25(\mathrm{~d}, J=8.5 \mathrm{~Hz}, 2 \mathrm{H}), 3.56(\mathrm{t}, J=6.0 \mathrm{~Hz}, 2 \mathrm{H}), 2.30(\mathrm{t}, J=6.8 \mathrm{~Hz}, 2 \mathrm{H}), 1.63$ $-1.59(\mathrm{~m}, 4 \mathrm{H}) ;{ }^{13} \mathrm{C}$ NMR $\left(75 \mathrm{MHz}, \mathrm{CDCl}_{3}, 300 \mathrm{~K}\right): \delta(\mathrm{ppm})=168.2(\mathrm{C}), 154.6(\mathrm{C})$, $134.0(\mathrm{CH}), 132.5(\mathrm{CH}), 131.9(\mathrm{C}), 130.2(\mathrm{CH}), 128.4(\mathrm{C}), 126.8(\mathrm{C}), 123.2(\mathrm{CH}), 113.8$ (C), 118.1 (q, J=318.8 Hz, CF $), 109.4(\mathrm{C}), 37.0\left(\mathrm{CH}_{2}\right), 29.0\left(\mathrm{CH}_{2}\right), 27.5\left(\mathrm{CH}_{2}\right), 25.1$ $\left(\mathrm{CH}_{2}\right) ;{ }^{19}$ F NMR $\left(282 \mathrm{MHz}, \mathrm{CDCl}_{3}, 300 \mathrm{~K}\right): \delta(\mathrm{ppm})=-73.3(\mathrm{~s}, 3 \mathrm{~F}) ;$ HRMS (ESI) $\mathrm{m} / \mathrm{z}$ $=580.9787$ calcd. for $\mathrm{C}_{22} \mathrm{H}_{16} \mathrm{BrF}_{3} \mathrm{~N}_{2} \mathrm{O}_{5} \mathrm{SNa}[\mathrm{M}+\mathrm{Na}]^{+}$, found: 580.9796 ; IR (neat, $\mathrm{cm}^{-}$ $\left.{ }^{1}\right): 2926 w, 2856 w, 2227 w, 1771 w, 1707 s, 1642 w, 1588 w, 1397 s, 1214 s, 1133 s, 1072 m$, $1018 m, 995 s, 912 m, 839 s, 720 s$.<smiles>CC(C#N)=C(OBr)c1ccc(Br)cc1</smiles>

\section{(Z)-1-(4-Bromophenyl)-2-cyanoprop-1-en-1-yl}

trifluoromethanesulfonate (2t): The title compound was prepared according to general procedure (GP2) with $\mathrm{Fe}(\mathrm{OAc})_{2}(3.5 \mathrm{mg}, 20$

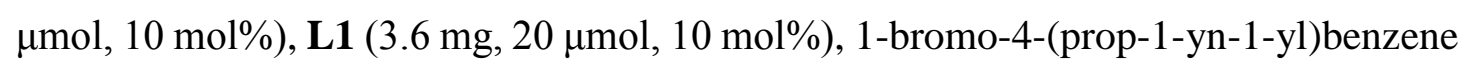
3t $\quad(38.8 \quad \mathrm{mg}, \quad 0.200 \quad$ mmol, $1.0 \quad$ equiv $), \quad$ and 3,5di(trifluoromethyl)phenyl(cyano)iodonium triflate $1 \mathbf{a}(227 \mathrm{mg}, 0.440 \mathrm{mmol}, 2.2$ equiv) in DCE $(1 \mathrm{~mL})$ at $45{ }^{\circ} \mathrm{C}$ for $15 \mathrm{~h}$. Purification via silica gel chromatography (Pentane:EtOAc $=200: 1$ ) gave the desired product $2 \mathrm{t}$ as a light yellow oil in $72 \%$ yield (52.7 mg). TLC $\mathbf{R}_{\mathrm{f}}=0.25$ (Pentane:EtOAc $\left.=7: 1\right) ;{ }^{1} \mathbf{H}$ NMR $\left(300 \mathrm{MHz}, \mathrm{CDCl}_{3}, 300 \mathrm{~K}\right)$ : $\delta(\mathrm{ppm})=7.59(\mathrm{~d}, J=8.4 \mathrm{~Hz}, 2 \mathrm{H}), 7.26(\mathrm{~d}, J=8.5 \mathrm{~Hz}, 2 \mathrm{H}), 2.00(\mathrm{~s}, 3 \mathrm{H}) ;{ }^{13} \mathbf{C ~ N M R}$ $\left(75 \mathrm{MHz}, \mathrm{CDCl}_{3}, 300 \mathrm{~K}\right): \delta(\mathrm{ppm})=154.5(\mathrm{C}), 132.5(\mathrm{CH}), 130.2(\mathrm{CH}), 128.5(\mathrm{C})$, $126.8(\mathrm{C}), 118.1$ (q, $\left.J=320.9 \mathrm{~Hz}, \mathrm{CF}_{3}\right), 114.9(\mathrm{C}), 104.4(\mathrm{C}), 16.5\left(\mathrm{CH}_{3}\right) ;{ }^{19} \mathbf{F}$ NMR $\left(282 \mathrm{MHz}, \mathrm{CDCl}_{3}, 300 \mathrm{~K}\right): \delta(\mathrm{ppm})=-73.3(\mathrm{~s}, 3 \mathrm{~F}) ;$ HRMS (ESI) $\mathrm{m} / \mathrm{z}=391.9174$, calcd. for $\mathrm{C}_{11} \mathrm{H}_{7} \mathrm{BrF}_{3} \mathrm{NO}_{3} \mathrm{SNa}[\mathrm{M}+\mathrm{Na}]^{+}$, found: 391.9177; IR (neat, $\mathrm{cm}^{-1}$ ): 2929w, 2228w, $1647 w, 1588 w, 1485 w, 1424 s, 1209 s, 1132 s, 1052 s, 987 s, 836 s, 766 m, 733 m, 686 w$, $601 s$. 
<smiles>N#C/C(=C\C(=O)c1ccc(Br)cc1)c1ccccc1</smiles>

fluoromethanesulfonate $(\mathbf{2 u})$ : The title compound was prepared according to general procedure $(\mathbf{G P 2})$ with $\mathrm{Fe}(\mathrm{OAc})_{2}(3.5 \mathrm{mg}, 20$

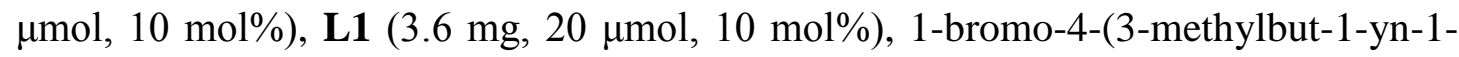
yl)benzene $\quad 3 \mathbf{u} \quad(44.6 \quad \mathrm{mg}, \quad 0.200 \quad \mathrm{mmol}, \quad 1.0$ equiv), and 3,5di(trifluoromethyl)phenyl(cyano)iodonium triflate $1 \mathbf{a}(227 \mathrm{mg}, 0.440 \mathrm{mmol}, 2.2$ equiv) in DCE $(1 \mathrm{~mL})$ at $45^{\circ} \mathrm{C}$ for $15 \mathrm{~h}$. Purification via silica gel chromatography (Pentane:EtOAc $=300: 1$, then 200:1) gave the desired product $\mathbf{2 u}$ as a white solid in $81 \%$ yield (64.6 mg). $\mathbf{T L C} \mathbf{R}_{\mathrm{f}}=0.35$ (Pentane:EtOAc $=40: 1$ ); MP: $66{ }^{\circ} \mathrm{C} ;{ }^{1} \mathbf{H}$ NMR $\left(300 \mathrm{MHz}, \mathrm{CDCl}_{3}, 300 \mathrm{~K}\right): \delta(\mathrm{ppm})=7.58(\mathrm{~d}, J=8.4 \mathrm{~Hz}, 2 \mathrm{H}), 7.23(\mathrm{~d}, J=8.4 \mathrm{~Hz}, 2 \mathrm{H})$, $2.65-2.54(\mathrm{~m}, 1 \mathrm{H}), 1.15(\mathrm{~d}, J=6.7 \mathrm{~Hz}, 6 \mathrm{H}) ;{ }^{13} \mathrm{C} \mathrm{NMR}\left(75 \mathrm{MHz}, \mathrm{CDCl}_{3}, 300 \mathrm{~K}\right)$ : $152.9(\mathrm{C}), 132.5(\mathrm{CH}), 130.0(\mathrm{CH}), 128.7(\mathrm{C}), 126.7(\mathrm{C}), 118.1$ (q, $\left.J=320.9 \mathrm{~Hz}, \mathrm{CF}_{3}\right)$, $116.8(\mathrm{C}), 112.6(\mathrm{C}), 29.2(\mathrm{CH}), 21.2\left(\mathrm{CH}_{3}\right) ;{ }^{19} \mathbf{F}$ NMR $\left(282 \mathrm{MHz}, \mathrm{CDCl}_{3}, 300 \mathrm{~K}\right): \delta$ $(\mathrm{ppm})=-73.4(\mathrm{~s}, 3 \mathrm{~F}) ;$ HRMS $(\mathrm{ESI}) \mathrm{m} / \mathrm{z}=421.9467$, calcd. for $\mathrm{C}_{13} \mathrm{H}_{11} \mathrm{BrF}_{3} \mathrm{NO}_{3} \mathrm{~S}$ $[\mathrm{M}+\mathrm{Na}]^{+}$, found: 421.9465; IR (neat, $\mathrm{cm}^{-1}$ ): 2976w, 2254w, 1629w, 1429w, 1224m, $1135 w, 1073 w, 998 m, 904 s, 834 w, 724 s, 649 s$.

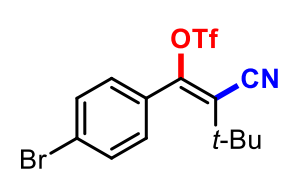

(Z)-1-(4-Bromophenyl)-2-cyano-3,3-dimethylbut-1-en-1-yl trifluoromethanesulfonate $(2 \mathrm{v})$ : The title compound was prepared according to general procedure (GP2) with $\mathrm{Fe}(\mathrm{OAc})_{2}(3.5 \mathrm{mg}, 20$

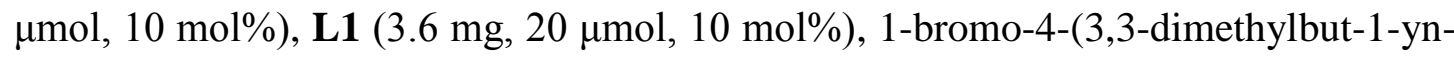
1-yl)benzene $\quad 3 v \quad(47.4 \quad \mathrm{mg}, \quad 0.200 \quad \mathrm{mmol}, \quad 1.0 \quad$ equiv), and 3,5di(trifluoromethyl)phenyl(cyano)iodonium triflate $1 \mathrm{a}(227 \mathrm{mg}, 0.440 \mathrm{mmol}, 2.2$ equiv) in DCE $(1 \mathrm{~mL})$ at $45^{\circ} \mathrm{C}$ for $15 \mathrm{~h}$. Then further portions of $\mathrm{Fe}(\mathrm{OAc})_{2}(3.5 \mathrm{mg}, 20 \mu \mathrm{mol}$, $10 \mathrm{~mol} \%), \quad \mathbf{L 1} \quad(3.6 \quad \mathrm{mg}, \quad 20 \quad \mu \mathrm{mol}, 10 \quad \mathrm{~mol} \%), \quad 3,5-$ di(trifluoromethyl)phenyl(cyano)iodonium triflate 1a (227 mg, $0.440 \mathrm{mmol}, 2.2$ equiv), and DCE $(1 \mathrm{~mL})$ were added. The stirring was continued at $45{ }^{\circ} \mathrm{C}$ for further $15 \mathrm{~h}$. Purification via silica gel chromatography (Pentane:EtOAc $=300: 1)$ gave the desired product $2 \mathrm{v}$ as a light yellow oil in $60 \%$ yield $\left(49.0 \mathrm{mg}\right.$ ). $\mathbf{T L C} \mathbf{R}_{\mathbf{f}}=0.55$ (Pentane:EtOAc $=20: 1) ;{ }^{1} \mathbf{H}$ NMR $\left(300 \mathrm{MHz}, \mathrm{CDCl}_{3}, 300 \mathrm{~K}\right): \delta(\mathrm{ppm})=7.55(\mathrm{~d}, J=8.3 \mathrm{~Hz}, 2 \mathrm{H}), 7.23$ 
$(\mathrm{d}, J=8.3 \mathrm{~Hz}, 2 \mathrm{H}), 1.06(\mathrm{~s}, 9 \mathrm{H}) ;{ }^{13} \mathbf{C ~ N M R}\left(75 \mathrm{MHz}, \mathrm{CDCl}_{3}, 300 \mathrm{~K}\right): \delta(\mathrm{ppm})=154.1$ (C), $132.0(\mathrm{CH}), 131.6(\mathrm{CH}), 129.4(\mathrm{C}), 126.6(\mathrm{C}), 121.7(\mathrm{C}), 118.0(\mathrm{q}, J=321.0 \mathrm{~Hz}$, $\left.\mathrm{CF}_{3}\right), 114.1(\mathrm{C}), 34.9(\mathrm{C}), 30.3\left(\mathrm{CH}_{3}\right) ;{ }^{19} \mathbf{F} \mathbf{N M R}\left(282 \mathrm{MHz}, \mathrm{CDCl}_{3}, 300 \mathrm{~K}\right): \delta(\mathrm{ppm})=$ -73.8 (s, 3F); HRMS (ESI) $m / z=435.9623$, calcd. for $\mathrm{C}_{14} \mathrm{H}_{13} \mathrm{BrF}_{3} \mathrm{NO}_{3} \mathrm{SNa}[\mathrm{M}+\mathrm{Na}]^{+}$, found: 435.9619; IR (neat, $\mathrm{cm}^{-1}$ ): 2975w, 2232w, 1636w, 1586w, 1481w, 1423s, 1209s, $1134 s, 1071 m, 1014 m, 963 s, 893 m, 842 s, 787 m, 758 m, 703 m$.

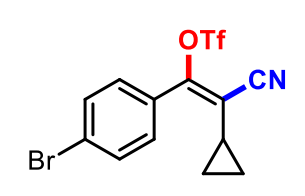

\section{(Z)-1-(4-Bromophenyl)-2-cyano-2-cyclopropylvinyl trifluo-} romethanesulfonate $(\mathbf{2 w})$ : The title compound was prepared according to general procedure (GP2) with $\mathrm{Fe}(\mathrm{OAc})_{2}(3.5 \mathrm{mg}, 20$ $\mu \mathrm{mol}, \quad 10 \quad \mathrm{~mol} \%), \quad \mathbf{L 1} \quad(3.6 \quad \mathrm{mg}, \quad 20 \mu \mathrm{mol}, \quad 10 \quad \mathrm{~mol} \%)$, 1-bromo-4(cyclopropylethynyl)benzene $\mathbf{3 w}$ (44.2 $\mathrm{mg}, 0.200 \mathrm{mmol}, 1.0$ equiv), and 3,5di(trifluoromethyl)phenyl(cyano)iodonium triflate $1 \mathbf{a}(227 \mathrm{mg}, 0.440 \mathrm{mmol}, 2.2$ equiv) in DCE $(1 \mathrm{~mL})$ at $45{ }^{\circ} \mathrm{C}$ for $15 \mathrm{~h}$. Purification via silica gel chromatography (Pentane:EtOAc $=300: 1$ ) gave the desired product $\mathbf{2 w}$ as a colorless oil in $42 \%$ yield (32.6 mg). TLC $\mathbf{R}_{\mathrm{f}}=0.45$ (Pentane:EtOAc $\left.=20: 1\right)$; ${ }^{1} \mathbf{H} \mathbf{N M R}\left(300 \mathrm{MHz}, \mathrm{CDCl}_{3}, 300\right.$ $\mathrm{K}): \delta(\mathrm{ppm})=7.59(\mathrm{~d}, J=8.4 \mathrm{~Hz}, 2 \mathrm{H}), 7.39(\mathrm{~d}, J=8.6 \mathrm{~Hz}, 2 \mathrm{H}), 1.61-1.52(\mathrm{~m}, 1 \mathrm{H})$, $0.93-0.90(\mathrm{~m}, 4 \mathrm{H}) ;{ }^{13} \mathrm{C}$ NMR (75 MHz, $\left.\mathrm{CDCl}_{3}, 300 \mathrm{~K}\right)$ : $153.0(\mathrm{C}), 132.4(\mathrm{CH}), 130.4$ (CH), $129.0(\mathrm{C}), 126.5(\mathrm{C}), 118.1$ (q, J = $\left.321.2 \mathrm{~Hz}, \mathrm{CF}_{3}\right), 113.2$ (C), 112.1 (C), 10.7 $(\mathrm{CH}), 7.9\left(\mathrm{CH}_{2}\right) ;{ }^{19} \mathbf{F}$ NMR $\left(282 \mathrm{MHz}, \mathrm{CDCl}_{3}, 300 \mathrm{~K}\right): \delta(\mathrm{ppm})=-73.3(\mathrm{~s}, 3 \mathrm{~F}) ; \mathbf{H R M S}$ (ESI) $m / z=419.9310$, calcd. for $\mathrm{C}_{13} \mathrm{H}_{9} \mathrm{BrF}_{3} \mathrm{NO}_{3} \mathrm{~S}[\mathrm{M}+\mathrm{Na}]^{+}$, found: 419.9314; IR (neat, $\left.\mathrm{cm}^{-1}\right): 3093 w, 2228 w, 1629 w, 1588 w, 1486 s, 1426 s, 1363 w, 1216 s, 1134 s, 1072 m$, $1002 s, 915 m, 869 m, 835 m, 798 m, 759 m, 684 w, 610 m$.

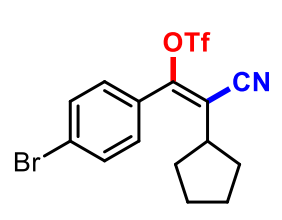

\section{(Z)-1-(4-Bromophenyl)-2-cyano-2-cyclopentylvinyl trifluoro-} methanesulfonate (2x): The title compound was prepared according to general procedure (GP2) with $\mathrm{Fe}(\mathrm{OAc})_{2}(3.5 \mathrm{mg}, 20$ $\mu \mathrm{mol}, \quad 10$ mol\%), $\quad \mathbf{L 1} \quad(3.6 \mathrm{mg}, \quad 20 \quad \mu \mathrm{mol}, \quad 10$ mol\%), 1-bromo-4(cyclopentylethynyl)benzene 3x (49.8 mg, $0.200 \mathrm{mmol}, 1.0$ equiv), and 3,5di(trifluoromethyl)phenyl(cyano)iodonium triflate $1 \mathbf{a}(227 \mathrm{mg}, 0.440 \mathrm{mmol}, 2.2$ equiv) 
in DCE $(1 \mathrm{~mL})$ at $45^{\circ} \mathrm{C}$ for $15 \mathrm{~h}$. Purification via silica gel chromatography (Pentane:EtOAc $=300: 1$ ) gave the desired product $\mathbf{2 x}$ as a colorless oil in $70 \%$ yield (58.8 mg). TLC $\mathbf{R}_{\mathrm{f}}=0.4$ (Pentane:EtOAc $\left.=40: 1\right) ;{ }^{1} \mathbf{H}$ NMR $\left(300 \mathrm{MHz}, \mathrm{CDCl}_{3}, 300 \mathrm{~K}\right)$ : $\delta(\mathrm{ppm})=7.57(\mathrm{~d}, J=8.4 \mathrm{~Hz}, 2 \mathrm{H}), 7.24(\mathrm{~d}, J=8.4 \mathrm{~Hz}, 2 \mathrm{H}), 2.58(\mathrm{p}, J=8.3 \mathrm{~Hz}, 1 \mathrm{H})$, $1.83-1.50(\mathrm{~m}, 8 \mathrm{H}) ;{ }^{13} \mathrm{C}$ NMR $\left(75 \mathrm{MHz}, \mathrm{CDCl}_{3}, 300 \mathrm{~K}\right): \delta(\mathrm{ppm})=153.1(\mathrm{C}), 132.5$ $(\mathrm{CH}), 130.2(\mathrm{CH}), 128.9(\mathrm{C}), 126.6(\mathrm{C}), 118.1$ (q, $\left.J=321.0 \mathrm{~Hz}, \mathrm{CF}_{3}\right), 115.1(\mathrm{C}), 113.2$ (C), $39.4(\mathrm{CH}), 32.6\left(\mathrm{CH}_{2}\right), 25.6\left(\mathrm{CH}_{2}\right) ;{ }^{19} \mathbf{F}$ NMR $\left(282 \mathrm{MHz}, \mathrm{CDCl}_{3}, 300 \mathrm{~K}\right): \delta(\mathrm{ppm})$ $=-73.4(\mathrm{~s}, 3 \mathrm{~F})$; HRMS $(\mathrm{ESI}) \mathrm{m} / \mathrm{z}=447.9624$, calcd. for $\mathrm{C}_{15} \mathrm{H}_{13} \mathrm{BrF}_{3} \mathrm{NO}_{3} \mathrm{~S}[\mathrm{M}+\mathrm{Na}]^{+}$, found: 447.9628; IR (neat, $\mathrm{cm}^{-1}$ ): 2959w, 2872w, 2226w, 1638w, 1588w, 1486w, 1425s, $1212 s, 1134 s, 1072 m, 1001 s, 871 m, 825 s, 759 w, 684 w$.

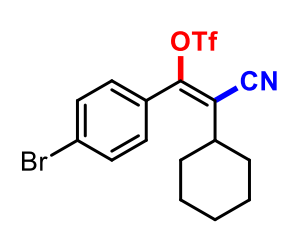

(Z)-1-(4-Bromophenyl)-2-cyano-2-cyclohexylvinyl trifluoromethanesulfonate (2y): The title compound was prepared according to general procedure (GP2) with $\mathrm{Fe}(\mathrm{OAc})_{2}(3.5 \mathrm{mg}, 20$ $\mu \mathrm{mol}, 10 \mathrm{~mol} \%)$, L1 (3.6 mg, $20 \mu \mathrm{mol}, 10 \mathrm{~mol} \%$ ), 1-bromo-4(cyclohexylethynyl)benzene $\mathbf{3 y}$ (52.6 $\mathrm{mg}, 0.200 \mathrm{mmol}, 1.0$ equiv), and 3,5di(trifluoromethyl)phenyl(cyano)iodonium triflate 1a $(227 \mathrm{mg}, 0.440 \mathrm{mmol}, 2.2$ equiv) in DCE $(1 \mathrm{~mL})$ at $45{ }^{\circ} \mathrm{C}$ for $15 \mathrm{~h}$. Purification via silica gel chromatography (Pentane:EtOAc $=300: 1)$ gave the desired product $\mathbf{2 y}$ as a colorless oil in $60 \%$ yield (52.6 mg). TLC $\mathbf{R}_{\mathrm{f}}=0.45$ (Pentane:EtOAc $\left.=40: 1\right) ;{ }^{1} \mathbf{H}$ NMR (300 MHz, $\mathrm{CDCl}_{3}, 300$ $\mathrm{K}): \delta(\mathrm{ppm})=7.59(\mathrm{~d}, J=8.4 \mathrm{~Hz}, 2 \mathrm{H}), 7.23(\mathrm{~d}, J=8.4 \mathrm{~Hz}, 2 \mathrm{H}), 2.21\left(\mathrm{tt}, J^{1}=11.7 \mathrm{~Hz}\right.$, $\left.J^{2}=3.5 \mathrm{~Hz}, 1 \mathrm{H}\right), 1.75-1.04(\mathrm{~m}, 10 \mathrm{H}) ;{ }^{13} \mathrm{C} \mathrm{NMR}\left(75 \mathrm{MHz}, \mathrm{CDCl}_{3}, 300 \mathrm{~K}\right): \delta(\mathrm{ppm})$ $=153.3(\mathrm{C}), 132.5(\mathrm{CH}), 130.0(\mathrm{CH}), 128.9(\mathrm{C}), 126.7(\mathrm{C}), 118.1(\mathrm{q}, J=321.0 \mathrm{~Hz}$, $\left.\mathrm{CF}_{3}\right), 115.8(\mathrm{C}), 113.2(\mathrm{C}), 38.6(\mathrm{CH}), 31.3\left(\mathrm{CH}_{2}\right), 25.3\left(\mathrm{CH}_{2}\right), 24.9\left(\mathrm{CH}_{2}\right) ;{ }^{19}$ F NMR $\left(282 \mathrm{MHz}, \mathrm{CDCl}_{3}, 300 \mathrm{~K}\right): \delta(\mathrm{ppm})=-73.4(\mathrm{~s}, 3 \mathrm{~F}) ; \mathbf{H R M S}(\mathrm{ESI}) \mathrm{m} / \mathrm{z}=461.9780$, calcd for $\mathrm{C}_{16} \mathrm{H}_{15} \mathrm{BrF}_{3} \mathrm{NO}_{3} \mathrm{SNa}[\mathrm{M}+\mathrm{Na}]^{+}$, found: 461.9782; IR (neat, $\mathrm{cm}^{-1}$ ): 2933m, 2857w, $2225 w, 1639 w, 1588 w, 1486 w, 1425 s, 1210 s, 1133 s, 1072 m, 1004 s, 982 s, 909 w, 835 s$, $787 m, 763 m, 685 w$. 


\section{Screening of reaction conditions}<smiles>PC#CC#Cc1ccc(Cl)cc1</smiles>

$3 c$

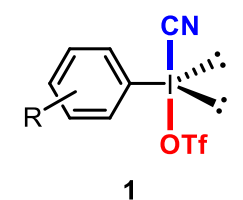
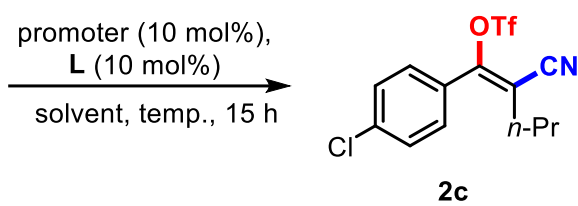

2c

\begin{tabular}{|c|c|c|c|c|c|c|}
\hline entry $^{a}$ & promotor & ligand & 1 & solvent & temperature & yield $(\%)^{b}$ \\
\hline 1 & $\mathrm{Fe}(\mathrm{OAc})_{2}$ & L1 & $1 \mathrm{a}$ & DCE & $45^{\circ} \mathrm{C}$ & $78,81^{c},(72: 1)$ \\
\hline 2 & none & none & $1 \mathrm{a}$ & DCE & $45^{\circ} \mathrm{C}$ & trace, (NA) \\
\hline 3 & $\mathrm{Fe}(\mathrm{OAc})_{2}$ & none & $1 \mathrm{a}$ & DCE & $45^{\circ} \mathrm{C}$ & $36,(26: 1)$ \\
\hline 4 & $\mathrm{Fe}(\mathrm{OAc})_{2}$ & L2 & $1 \mathrm{a}$ & DCE & $45^{\circ} \mathrm{C}$ & $51,(61: 1)$ \\
\hline 5 & $\mathrm{Fe}(\mathrm{OAc})_{2}$ & L3 & $1 \mathrm{a}$ & DCE & $45^{\circ} \mathrm{C}$ & $20,(24: 1)$ \\
\hline 6 & $\mathrm{Fe}(\mathrm{OAc})_{2}$ & L4 & $1 \mathrm{a}$ & DCE & $45^{\circ} \mathrm{C}$ & $24,(18: 1)$ \\
\hline 7 & $\mathrm{Fe}(\mathrm{OAc})_{2}$ & L5 & $1 \mathrm{a}$ & DCE & $45^{\circ} \mathrm{C}$ & $31,(23: 1)$ \\
\hline 8 & $\mathrm{Fe}(\mathrm{OAc})_{2}$ & L1 & $1 b$ & DCE & $45^{\circ} \mathrm{C}$ & $22,(22: 1)$ \\
\hline 9 & $\mathrm{Fe}(\mathrm{OAc})_{2}$ & L1 & 1c & DCE & $45^{\circ} \mathrm{C}$ & $40,(54: 1)$ \\
\hline 10 & $\mathrm{Fe}(\mathrm{OAc})_{2}$ & L1 & $1 \mathrm{a}$ & $\mathrm{DCM}$ & $45^{\circ} \mathrm{C}$ & $63,(42: 1)$ \\
\hline 11 & $\mathrm{Fe}(\mathrm{OAc})_{2}$ & L1 & $1 \mathrm{a}$ & $\mathrm{MeCN}$ & $45^{\circ} \mathrm{C}$ & trace, (NA) \\
\hline 12 & $\mathrm{Fe}(\mathrm{OAc})_{2}$ & L1 & $1 \mathrm{a}$ & DCE & rt. & $64,(91: 1)$ \\
\hline 13 & $\mathrm{Fe}(\mathrm{OTf})_{2}$ & L1 & $1 \mathrm{a}$ & DCE & $45^{\circ} \mathrm{C}$ & $23,(4: 1)$ \\
\hline 14 & $\mathrm{FeCl}_{2}$ & L1 & $1 \mathrm{a}$ & DCE & $45^{\circ} \mathrm{C}$ & $49,(15: 1)$ \\
\hline 15 & $\mathrm{FeCl}_{3}$ & L1 & $1 \mathrm{a}$ & DCE & $45^{\circ} \mathrm{C}$ & $55,(21: 1)$ \\
\hline 16 & $\mathrm{CuCl}$ & none & $1 \mathrm{a}$ & DCE & $45^{\circ} \mathrm{C}$ & trace, (NA) \\
\hline 17 & $\mathrm{BF}_{3} \cdot \mathrm{Et}_{2} \mathrm{O}$ & none & $1 a$ & DCE & $45^{\circ} \mathrm{C}$ & $12,(\mathrm{NA})$ \\
\hline 18 & HOTf & none & $1 \mathrm{a}$ & DCE & $45^{\circ} \mathrm{C}$ & trace, (NA) \\
\hline 19 & $\mathrm{AlCl}_{3}$ & none & $1 \mathrm{a}$ & DCE & $45^{\circ} \mathrm{C}$ & $12,(2: 1)$ \\
\hline 20 & TBAI & none & $1 a$ & DCE & $45^{\circ} \mathrm{C}$ & $32,(9: 1)$ \\
\hline
\end{tabular}

a Reaction condition: $3 c(0.20 \mathrm{mmol}, 1.0$ equiv. $)$, reagent $1(0.44 \mathrm{mmol}, 2.2$ equiv.), promoter $(0.02 \mathrm{mmol}, 10 \mathrm{~mol} \%)$, ligand $(0.02 \mathrm{mmol}, 10 \mathrm{~mol} \%)$, solvent $(1 \mathrm{~mL}), 45^{\circ} \mathrm{C}, 15 \mathrm{~h}$. ${ }^{b}$ Yield determined by ${ }^{19} \mathrm{~F} \mathrm{NMR}$ analysis using $\mathrm{PhCF}_{3}$ as an internal standard; isomer ratio in parentheses determined by GC-MS analysis on the crude product; NA, not applicable; cIsolated yield.

ligand $=$

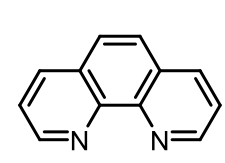

L1<smiles>Cc1cccc(C)n1</smiles>

L2
L3<smiles>CC(C)[C@H]1COC(c2cccc(C3=NC(P)CO3)n2)=N1</smiles>

14<smiles>c1ccc(-c2cccc(-c3ccccn3)n2)nc1</smiles>

L5

A flame-dried Schlenk-tube equipped with a magnetic stir bar was charged with promoter $(0.020 \mathrm{mmol}, 10 \mathrm{mmol} \%)$ and ligand $(0.020 \mathrm{mmol}, 10 \mathrm{mmol} \%)$, sealed with a septum, and degassed by alternating vacuum evacuation and argon backfilling (three times) before DCE ( $1 \mathrm{~mL})$ was added. The resulting suspension was stirred for $5 \mathrm{~min}$ 
at room temperature. 1-Chloro-4-(pent-1-yn-1-yl)benzene 1c $(35.7 \mathrm{mg}, 0.200 \mathrm{mmol}$, 1.0 equiv) and 3,5-di(trifluoromethyl)phenyl(cyano)iodonium triflate $\mathbf{1 a}(0.440 \mathrm{mmol}$, $227 \mathrm{mg}, 2.2$ equiv) were added successively under a flow of argon. The reaction mixture was then stirred at $45{ }^{\circ} \mathrm{C}$ for $15 \mathrm{~h}$. After the reaction was complete, the solvent was removed under reduced pressure with the aid of a rotary evaporator. The crude residue was analyzed by GC-MS and ${ }^{19} \mathrm{~F}$ NMR.

\section{Mechanistic study}

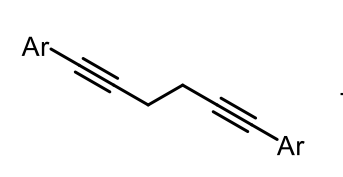

$3 \mathbf{z}$ $\mathrm{Ar}=4-\mathrm{ClC}_{6} \mathrm{H}_{4}$

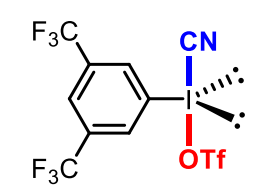

1a

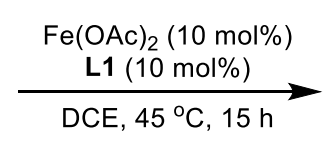

DCE, $45^{\circ} \mathrm{C}, 15 \mathrm{~h}$

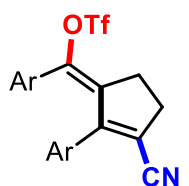

$2 \mathbf{z}, 34 \%$<smiles>N#CC1=C(c2ccc(Cl)cc2)/C(=C(\O)c2ccc(Cl)cc2)CC1</smiles>

(E)-(4-Chlorophenyl)(2-(4-chlorophenyl)-3-cyanocyclopent-2en-1-ylidene)methyl trifluoromethanesulfonate (2z): The title compound was prepared according to general procedure (GP2) with $\mathrm{Fe}(\mathrm{OAc})_{2}(3.5 \mathrm{mg}, 20 \mu \mathrm{mol}, 10 \mathrm{~mol} \%)$, L1 (3.6 mg, $20 \mu \mathrm{mol}$, $10 \mathrm{~mol} \%$ ), 1,6-bis(4-chlorophenyl)hexa-1,5-diyne 3z (59.6 mg, $0.200 \mathrm{mmol}, 1.0$ equiv), and 3,5-di(trifluoromethyl)phenyl(cyano)iodonium triflate $1 \mathbf{a}(227 \mathrm{mg}, 0.440 \mathrm{mmol}$, 2.2 equiv) in DCE $(1 \mathrm{~mL})$ at $45^{\circ} \mathrm{C}$ for $15 \mathrm{~h}$. Purification via silica gel chromatography (Pentane:EtOAc = 100:1) gave the desired product $\mathbf{2 z}$ as an off-white solid in 34\% yield (32.1 mg). TLC $\mathbf{R}_{\mathrm{f}}=0.35$ (Pentane:EtOAc $\left.=10: 1\right)$; MP: $79^{\circ} \mathrm{C} ;{ }^{1} \mathbf{H}$ NMR $(300 \mathrm{MHz}$, $\left.\mathrm{CDCl}_{3}, 300 \mathrm{~K}\right): \delta(\mathrm{ppm})=6.93\left(\mathrm{dd}, J^{1}=10.9 \mathrm{~Hz}, J^{2}=8.6 \mathrm{~Hz}, 4 \mathrm{H}\right), 6.74\left(\mathrm{dd}, J^{1}=16.0\right.$ $\left.\mathrm{Hz}, J^{2}=8.5 \mathrm{~Hz}, 4 \mathrm{H}\right), 3.14-3.10(\mathrm{~m}, 2 \mathrm{H}), 2.89-2.85(\mathrm{~m}, 2 \mathrm{H}) ;{ }^{13} \mathbf{C}$ NMR $(75 \mathrm{MHz}$, $\left.\mathrm{CDCl}_{3}, 300 \mathrm{~K}\right): \delta(\mathrm{ppm})=153.6(\mathrm{C}), 143.3(\mathrm{C}), 139.0(\mathrm{C}), 136.6(\mathrm{C}), 135.2(\mathrm{C}), 131.0$ $(\mathrm{CH}), 129.5(\mathrm{CH}), 129.3(\mathrm{C}), 128.9(\mathrm{C}), 128.2(\mathrm{CH}), 128.0(\mathrm{CH}), 122.5(\mathrm{C}), 118.06$ (q, $\left.J=320.5 \mathrm{~Hz}, \mathrm{CF}_{3}\right), 115.7(\mathrm{C}), 32.2\left(\mathrm{CH}_{2}\right), 29.4\left(\mathrm{CH}_{2}\right) ;{ }^{19} \mathbf{F} \mathbf{N M R}\left(282 \mathrm{MHz}, \mathrm{CDCl}_{3}\right.$, $300 \mathrm{~K}): \delta(\mathrm{ppm})=-74.2(\mathrm{~s}, 3 \mathrm{~F})$; HRMS (ESI) $\mathrm{m} / \mathrm{z}=495.9759$, calcd. for $\mathrm{C}_{20} \mathrm{H}_{12} \mathrm{Cl}_{2} \mathrm{~F}_{3} \mathrm{NO}_{3} \mathrm{SNa}[\mathrm{M}+\mathrm{Na}]^{+}$, found: 495.9743; IR (neat, $\mathrm{cm}^{-1}$ ): 2934w, 2216w, 
$1648 w, 1594 w, 1489 m, 1412 s, 1347 w, 1213 s, 1136 s, 1092 m, 1018 m, 929 m, 859 s, 831 s$, $734 w, 667 w$.
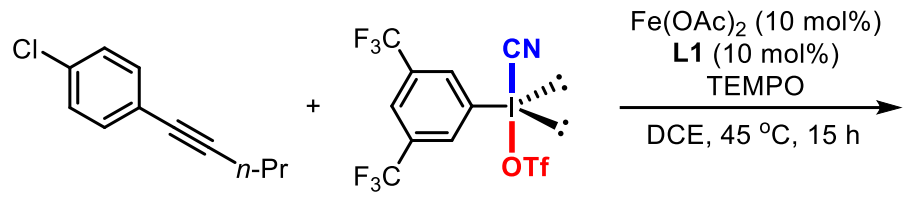<smiles>C/C=C(\C#N)C(=O)c1ccc(Cl)cc1</smiles>

A flame-dried Schlenk-tube equipped with a magnetic stir bar was charged with iron(II) acetate (3.5 mg, $20 \mu \mathrm{mol}, 10 \mathrm{~mol} \%$ ) and 1,10-phenanthroline L1 (3.6 mg, 20 $\mu \mathrm{mol}, 10 \mathrm{~mol} \%$ ), sealed with a septum, and degassed by alternating vacuum evacuation and argon backfilling (three times) before DCE $(1 \mathrm{~mL})$ was added. The resulting suspension was stirred for $5 \mathrm{~min}$ at room temperature. The corresponding alkyne 1-1chloro-4-(pent-1-yn-1-yl)benzene 1c (35.7 mg, $0.200 \mathrm{mmol}, 1.0$ equiv) and 3,5di(trifluoromethyl)phenyl(cyano)iodonium triflate $1 \mathbf{a}(227 \mathrm{mg}, 0.440 \mathrm{mmol}, 2.2$ equiv), and 2,2,6,6-Tetramethylpiperidine 1-oxyl (68.7 mg, $0.440 \mathrm{mmol}, 2.2$ equiv) were added successively under a flow of argon. The reaction mixture was then stirred at $45^{\circ} \mathrm{C}$ for 24 hours. After the reaction was complete, the solvent was removed under reduced pressure with the aid of a rotary evaporator. No desired product $\mathbf{2 c}$ and TEMPO-CN adduct was detected by GC-MS and ${ }^{19}$ F NMR analysis. 


\section{Derivatization of vinyl triflates}
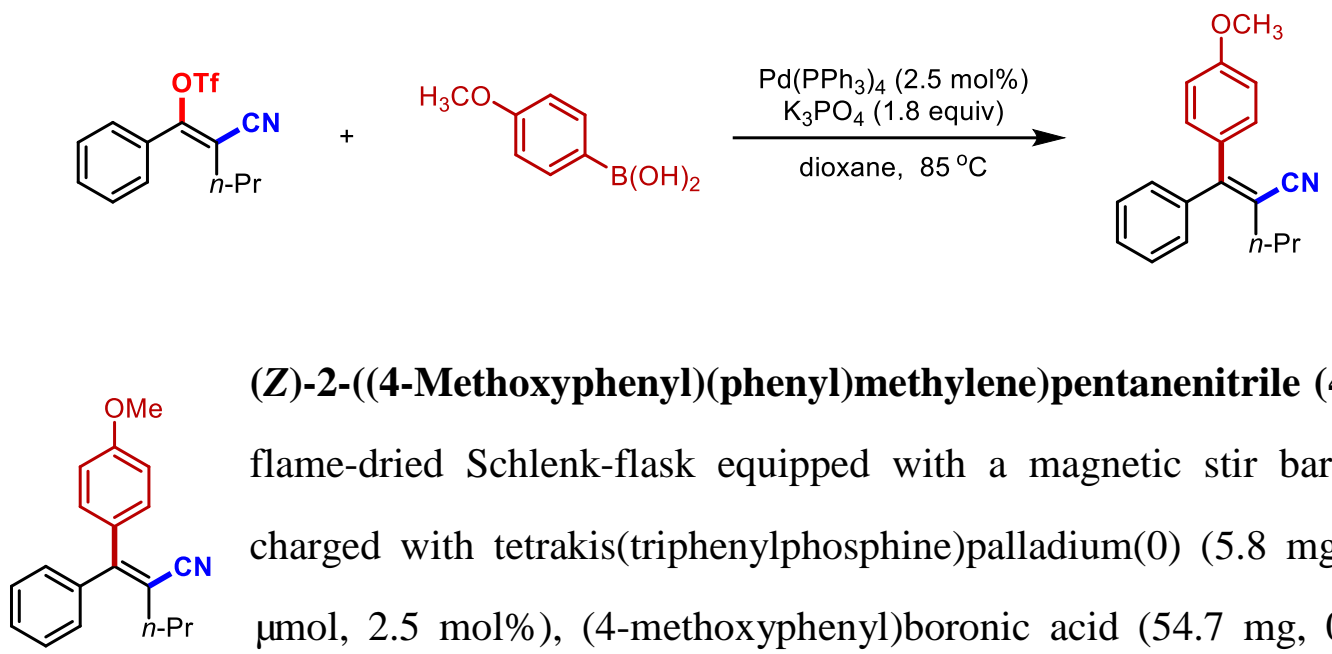

(Z)-2-((4-Methoxyphenyl)(phenyl)methylene)pentanenitrile (4): A flame-dried Schlenk-flask equipped with a magnetic stir bar was charged with tetrakis(triphenylphosphine)palladium(0) $(5.8 \mathrm{mg}, 5.0$ $\mu \mathrm{mol}, 2.5 \mathrm{~mol} \%$ ), (4-methoxyphenyl)boronic acid (54.7 mg, 0.360 mmol, 1.8 equiv), tripotassium phosphate ( $76.4 \mathrm{mg}, 0.360 \mathrm{mmol}, 1.8$ equiv), sealed with a septum, and degassed by alternating vacuum evacuation and argon backfilling (three times) before dioxane (2 mL) was added. (Z)-2-Cyano-1-phenylpent-1-en-1-yl trifluoromethanesulfonate $2 \mathbf{a}(63.9 \mathrm{mg}, 0.200 \mathrm{mmol}, 1.0$ equiv, $\mathrm{dr}>20: 1)$ was added to the resulting suspension subsequently. The reaction mixture was stirred at $85^{\circ} \mathrm{C}$ for 24 hours. After the reaction was complete, the reaction mixture was diluted with $\mathrm{Et}_{2} \mathrm{O}$ (10 $\mathrm{mL}$ ) and filtrated through a small pad of silica gel. The solvent was removed under reduced pressure with the aid of a rotary evaporator and the crude residue was purified by a silica gel column chromatography (Pentane:EtOAc $=200: 1)$ to give pure product 4 as a light yellow oil in $86 \%$ yield $\left(47.8 \mathrm{mg}\right.$, dr>20:1). TLC $\mathbf{R}_{\mathrm{f}}=0.55$ (Pentane:EtOAc $=10: 1) ;{ }^{1} \mathbf{H}$ NMR $\left(300 \mathrm{MHz}, \mathrm{CDCl}_{3}, 300 \mathrm{~K}\right): \delta(\mathrm{ppm})=7.30-7.25(\mathrm{~m}, 3 \mathrm{H}), 7.21(\mathrm{~d}$, $J=8.7 \mathrm{~Hz}, 2 \mathrm{H}), 7.04-7.01(\mathrm{~m}, 2 \mathrm{H}), 6.77(\mathrm{~d}, J=8.8 \mathrm{~Hz}, 2 \mathrm{H}), 3.71(\mathrm{~s}, 3 \mathrm{H}), 2.20(\mathrm{t}, J=$ $7.7 \mathrm{~Hz}, 2 \mathrm{H}), 1.59\left(\mathrm{tq}, J^{1}=7.5 \mathrm{~Hz}, J^{2}=7.5 \mathrm{~Hz}, 2 \mathrm{H}\right), 0.82(\mathrm{t}, J=7.3 \mathrm{~Hz}, 3 \mathrm{H}) ;{ }^{13} \mathbf{C} \mathbf{N M R}$ $\left(75 \mathrm{MHz}, \mathrm{CDCl}_{3}, 300 \mathrm{~K}\right): \delta(\mathrm{ppm})=160.3(\mathrm{C}), 156.7(\mathrm{C}), 139.2(\mathrm{C}), 132.2(\mathrm{C}), 130.8$ $(\mathrm{CH}), 129.1(\mathrm{CH}), 128.5(\mathrm{CH}), 128.3(\mathrm{CH}), 120.1(\mathrm{C}), 113.6(\mathrm{CH}), 110.5(\mathrm{C}), 55.2$ $\left(\mathrm{CH}_{3}\right), 34.1\left(\mathrm{CH}_{2}\right), 21.9\left(\mathrm{CH}_{2}\right), 13.3\left(\mathrm{CH}_{3}\right) ;$ HRMS (ESI) $\mathrm{m} / \mathrm{z}=300.1359$, calcd. for $\mathrm{C}_{19} \mathrm{H}_{19} \mathrm{NONa}[\mathrm{M}+\mathrm{Na}]^{+}$, found: 300.1354 ; IR (neat, $\mathrm{cm}^{-1}$ ): 2961w, 2205w, 1605s, 1509s, $1461 m, 1289 m, 1250 s, 1174 s, 1114 w, 1075 w, 1030 s, 908 w, 829 s, 759 m, 702 s, 656 w$. 


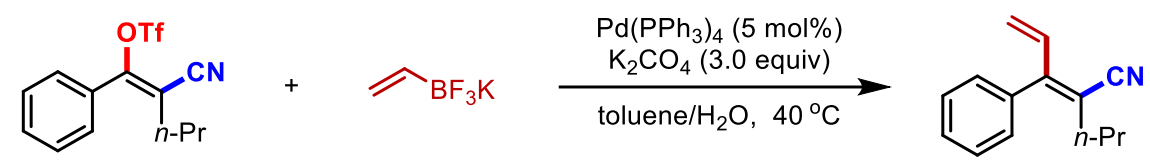

(E)-3-Phenyl-2-propylpenta-2,4-dienenitrile (5): A flame-dried<smiles>C=C/C(=C(\C#N)CC)c1ccccc1</smiles>

Schlenk-flask equipped with a magnetic stir bar was charged with tetrakis(triphenylphosphine)palladium(0) (14.4 mg, $12.5 \mu \mathrm{mol}, 5.0$ $\mathrm{mol} \%)$, potassium vinyltrifluoroborate $(53.5 \mathrm{mg}, 0.400 \mathrm{mmol}, 1.6$ equiv), potassium carbonate $(0.104 \mathrm{~g}, 0.750 \mathrm{mmol}, 3.0$ equiv), sealed with a septum, and degassed by alternating vacuum evacuation and argon backfilling (three times) before toluene $(5 \mathrm{~mL})$ and $\quad \mathrm{H}_{2} \mathrm{O} \quad(1 \quad \mathrm{~mL})$ was added. (Z)-2-Cyano-1-phenylpent-1-en-1-yl trifluoromethanesulfonate $\mathbf{2 a}(79.8 \mathrm{mg}, 0.250 \mathrm{mmol}, 1.0$ equiv, $\mathrm{dr}>20: 1)$ was added to the resulting suspension subsequently. The reaction mixture was stirred at $40{ }^{\circ} \mathrm{C}$ for 24 hours. After the reaction was complete, the reaction mixture was diluted with $\mathrm{Et}_{2} \mathrm{O}$ (10 $\mathrm{mL}$ ) and filtrated through a small pad of silica gel. The solvent was removed under reduced pressure with the aid of a rotary evaporator and the crude residue was purified by a silica gel column chromatography (Pentane:EtOAc $=300: 1)$ to give pure product $\mathbf{5}$ as a yellow oil in 90\% yield (44.4 mg, dr>20:1). $\mathbf{R}_{\mathrm{f}}=0.6($ Pentane:EtOAc $=20: 1) ;{ }^{1} \mathbf{H}$ NMR (300 MHz, $\left.\mathrm{CDCl}_{3}, 300 \mathrm{~K}\right): \delta(\mathrm{ppm})=7.37-7.26(\mathrm{~m}, 3 \mathrm{H}), 7.11\left(\mathrm{dd}, J^{1}=17.0\right.$ $\left.\mathrm{Hz}, J^{2}=10.5 \mathrm{~Hz}, 1 \mathrm{H}\right), 7.00-6.97(\mathrm{~m}, 2 \mathrm{H}), 5.38\left(\mathrm{dd}, J^{1}=10.5 \mathrm{~Hz}, J^{2}=0.8 \mathrm{~Hz}, 1 \mathrm{H}\right)$, $4.88\left(\mathrm{dd}, J^{1}=16.9 \mathrm{~Hz}, J^{2}=0.7 \mathrm{~Hz}, 1 \mathrm{H}\right), 1.96(\mathrm{t}, J=7.2 \mathrm{~Hz}, 2 \mathrm{H}), 1.47\left(\mathrm{tq}, J^{1}=7.5 \mathrm{~Hz}\right.$, $\left.J^{2}=7.5 \mathrm{~Hz}, 2 \mathrm{H}\right), 0.75(\mathrm{t}, J=7.4 \mathrm{~Hz}, 3 \mathrm{H}) ;{ }^{13} \mathbf{C ~ N M R}\left(75 \mathrm{MHz}, \mathrm{CDCl}_{3}, 300 \mathrm{~K}\right): \delta(\mathrm{ppm})$ $=154.4(\mathrm{C}), 136.4(\mathrm{CH}), 135.1(\mathrm{C}), 128.6(\mathrm{CH}), 128.4(\mathrm{CH}), 128.2(\mathrm{CH}), 123.8\left(\mathrm{CH}_{2}\right)$, $118.3(\mathrm{C}), 113.5(\mathrm{C}), 33.0\left(\mathrm{CH}_{2}\right), 21.5\left(\mathrm{CH}_{2}\right), 13.3\left(\mathrm{CH}_{3}\right)$; HRMS (ESI) $\mathrm{m} / \mathrm{z}=220.1097$, calcd. for $\mathrm{C}_{14} \mathrm{H}_{15} \mathrm{NNa}[\mathrm{M}+\mathrm{Na}]^{+}$, found: 220.1091; IR (neat, $\mathrm{cm}^{-1}$ ): 2963w, 2874w, $2208 w, 1574 w, 1461 w, 1409 w, 1312 w, 1260 w, 1088 w, 1027 w, 985 w, 928 m, 765 m$, $701 s$.

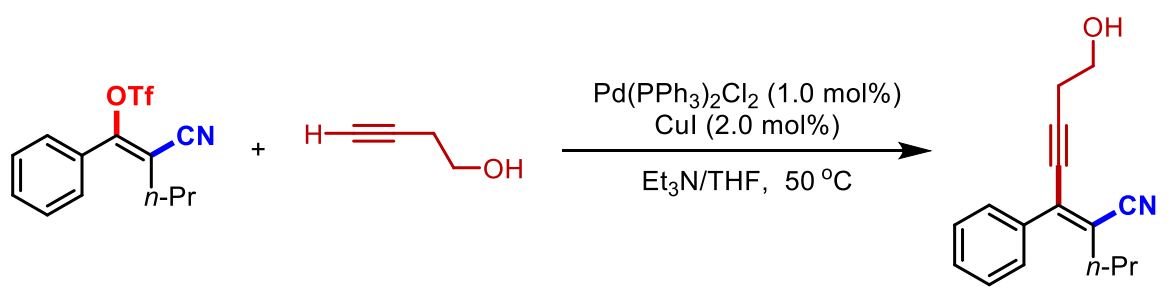


<smiles>CCCC#C/C(=C(\C#N)C#CCCO)c1ccccc1</smiles>

(Z)-7-Hydroxy-3-phenyl-2-propylhept-2-en-4-ynenitrile (6): A flame-dried Schlenk-flask equipped with a magnetic stir bar was charged with bis(triphenylphosphine)palladium(II) dichloride (1.4 mg, $2 \mu \mathrm{mol}, 1.0 \mathrm{~mol} \%)$, copper(I) iodide (0.8 mg, $4 \mu \mathrm{mol}, 2.0 \mathrm{~mol} \%$ ), sealed with a septum, and degassed by alternating vacuum evacuation and argon backfilling (three times) before tetrahydrofuran $(1 \mathrm{~mL})$ and triethylamine $(1$ $\mathrm{mL}$ ) was added. (Z)-2-Cyano-1-phenylpent-1-en-1-yl trifluoromethanesulfonate 2a (63.9 mg, $0.200 \mathrm{mmol}, 1.0$ equiv, dr>20:1) and but-3-yn-1-ol (28.0 mg, $0.400 \mathrm{mmol}$, 2.0 equiv) was added to the resulting suspension successively. The reaction mixture was stirred at $50{ }^{\circ} \mathrm{C}$ for 24 hours. After the reaction was complete, the reaction mixture was diluted with $\mathrm{Et}_{2} \mathrm{O}(10 \mathrm{~mL})$ and filtrated through a small pad of silica gel. The solvent was removed under reduced pressure with the aid of a rotary evaporator and the crude residue was purified by a silica gel column chromatography $($ Pentane:EtOAc $=$ $8: 1)$ to give pure product 6 as a light yellow oil in $84 \%$ yield $(40.2 \mathrm{mg}, \mathrm{dr}>20: 1)$. $\mathbf{R}_{\mathrm{f}}=$ 0.2 (Pentane:EtOAc $=4: 1) ;{ }^{1} \mathbf{H}$ NMR $\left(300 \mathrm{MHz}, \mathrm{CDCl}_{3}, 300 \mathrm{~K}\right): \delta(\mathrm{ppm})=7.39-7.30$ $(\mathrm{m}, 3 \mathrm{H}), 7.28-7.22(\mathrm{~m}, 2 \mathrm{H}), 3.74(\mathrm{t}, J=6.1 \mathrm{~Hz}, 2 \mathrm{H}), 2.62(\mathrm{t}, J=6.1 \mathrm{~Hz}, 2 \mathrm{H}), 2.26-$ $2.20(\mathrm{~m}, 3 \mathrm{H}), 1.54\left(\mathrm{tq}, J^{1}=7.5 \mathrm{~Hz}, J^{2}=7.5 \mathrm{~Hz}, 2 \mathrm{H}\right), 0.82(\mathrm{t}, J=7.3 \mathrm{~Hz}, 3 \mathrm{H}) ;{ }^{13} \mathbf{C}$ NMR $\left(75 \mathrm{MHz}, \mathrm{CDCl}_{3}, 300 \mathrm{~K}\right): \delta(\mathrm{ppm})=138.3(\mathrm{C}), 135.4(\mathrm{C}), 129.1(\mathrm{CH}), 128.5(\mathrm{CH})$, $128.3(\mathrm{CH}), 120.1(\mathrm{C}), 119.6(\mathrm{C}), 97.0(\mathrm{C}), 81.8(\mathrm{C}), 60.8\left(\mathrm{CH}_{2}\right), 32.2\left(\mathrm{CH}_{2}\right), 24.2$ $\left(\mathrm{CH}_{2}\right), 21.7\left(\mathrm{CH}_{2}\right), 13.3\left(\mathrm{CH}_{3}\right) ; \mathbf{H R M S}(\mathrm{ESI}) \mathrm{m} / z=262.1202$, calcd. for $\mathrm{C}_{16} \mathrm{H}_{17} \mathrm{NONa}$ $[\mathrm{M}+\mathrm{Na}]^{+}$, found: 262.1227; IR (neat, $\mathrm{cm}^{-1}$ ): 3418br, 3062w, 2926m, 2208m, 1718w, $1565 w, 1461 m, 1380 m, 1334 m, 1243 w, 1153 w, 1047 s, 962 w, 924 w, 846 w, 761 s, 700 s$.

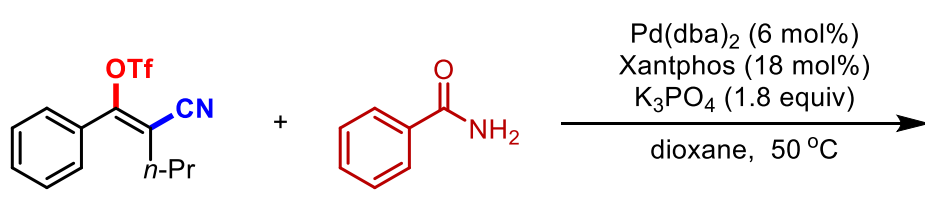


<smiles>N#C/C(C(=O)c1ccccc1)=C(\NC(=O)c1ccccc1)c1ccccc1</smiles>
(E)-N-(2-Cyano-1-phenylpent-1-en-1-yl)benzamide (7): A flamedried Schlenk-flask equipped with a magnetic stir bar was charged with bis(dibenzylideneacetone)palladium(0) $(6.9 \mathrm{mg}, 12 \mu \mathrm{mol}, 6.0$ mol\%), Xantphos (20.8 mg, $36.0 \mu \mathrm{mol}, 18 \mathrm{~mol} \%)$, tripotassium phosphate (0.076 g, $0.36 \mathrm{mmol}, 1.8$ equiv), and benzamide (36.3 mg, $0.300 \mathrm{mmol}, 1.5$ equiv), sealed with a septum, and degassed by alternating vacuum evacuation and argon backfilling (three times) before dioxane $(2 \mathrm{~mL}$ ) was added. $(Z)-2-C y a n o-1-$ phenylpent1-en-1-yl trifluoromethanesulfonate $\mathbf{2 a}(63.9 \mathrm{mg}, 0.200 \mathrm{mmol}, 1.0$ equiv, $\mathrm{dr}>20: 1)$ was added to the resulting suspension subsequently. The reaction mixture was stirred at 50 ${ }^{\circ} \mathrm{C}$ for 12 hours. After the reaction was complete, the reaction mixture was diluted with $\mathrm{Et}_{2} \mathrm{O}(10 \mathrm{~mL})$ and filtrated through a small pad of silica gel. The solvent was removed under reduced pressure with the aid of a rotary evaporator and the crude residue was purified by a silica gel column chromatography (Pentane:EtOAc $=12: 1$, then $8: 1$ ) to give pure product 7 as a yellow solid in $58 \%$ yield $(33.9 \mathrm{mg}$, containing $11 \%$ of imine tautomer). $\mathbf{R}_{\mathrm{f}}=0.25$ (Pentane:EtOAc $\left.=5: 1\right)$; enamine tautomer: ${ }^{1} \mathbf{H}$ NMR $(300 \mathrm{MHz}$, $\left.\mathrm{CDCl}_{3}, 300 \mathrm{~K}\right): \delta(\mathrm{ppm})=8.14(\mathrm{~s}, 1 \mathrm{H}), 7.78-7.75(\mathrm{~m}, 2 \mathrm{H}), 7.51-7.45(\mathrm{~m}, 1 \mathrm{H}), 7.39$ $\left(\mathrm{dd}, J^{1}=6.4 \mathrm{~Hz}, J^{2}=1.4 \mathrm{~Hz}, 2 \mathrm{H}\right), 7.36-7.32(\mathrm{~m}, 3 \mathrm{H}), 7.30-7.26(\mathrm{~m}, 2 \mathrm{H}), 2.23-2.06$ $(\mathrm{t}, J=7.5 \mathrm{~Hz}, 2 \mathrm{H}), 1.54\left(\mathrm{tq}, J^{1}=7.5 \mathrm{~Hz}, J^{2}=7.2 \mathrm{~Hz}, 2 \mathrm{H}\right), 0.83(\mathrm{t}, J=7.4 \mathrm{~Hz}, 2 \mathrm{H}) ;{ }^{13} \mathrm{C}$ NMR (75 MHz, $\left.\mathrm{CDCl}_{3}, 300 \mathrm{~K}\right): \delta(\mathrm{ppm})=165.0(\mathrm{C}), 149.2(\mathrm{C}), 133.7$ (C), $133.2(\mathrm{C})$, $132.6(\mathrm{CH}), 129.7(\mathrm{CH}), 128.8(\mathrm{CH}), 128.5(\mathrm{CH}), 128.3(\mathrm{CH}), 127.5(\mathrm{CH}), 118.2(\mathrm{C})$, $101.8(\mathrm{C}), 30.7\left(\mathrm{CH}_{2}\right), 22.1\left(\mathrm{CH}_{2}\right), 13.3\left(\mathrm{CH}_{3}\right)$; HRMS (ESI) $\mathrm{m} / \mathrm{z}=313.1311$, calcd. for $\mathrm{C}_{19} \mathrm{H}_{18} \mathrm{~N}_{2} \mathrm{ONa}[\mathrm{M}+\mathrm{Na}]^{+}$, found: 313.1317 ; IR (neat, $\mathrm{cm}^{-1}$ ): 3271br, 3062w, 2962w, $2929 w, 2873 w, 2208 w, 1661 s, 1603 m, 1479 s, 1270 s, 1151 w, 1076 w, 1027 w, 915 w$.

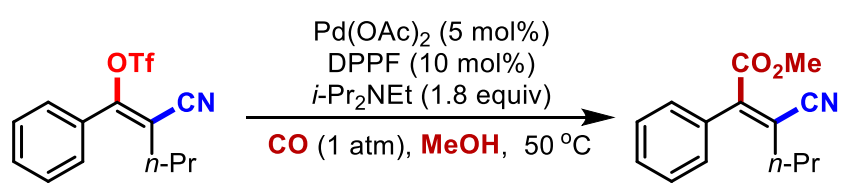


<smiles>CC(=O)C(=C(C#N)C(C)C)c1ccccc1</smiles>

Methyl (Z)-3-cyano-2-phenylhex-2-enoate (8): A flame-dried Schlenk-flask equipped with a magnetic stir bar was charged with palladium(II) acetate $(2.8 \mathrm{mg}, 13 \mu \mathrm{mol}, 5.0 \mathrm{~mol} \%)$, DPPF (13.9 mg, $25.0 \mu \mathrm{mol}, 10 \mathrm{~mol} \%$ ), sealed with a septum, and degassed by alternating vacuum evacuation and argon backfilling (three times) before methanol $(1.5 \mathrm{~mL})$ was added. $N$, $N$-diisopropylethylamine (32.3 $\mathrm{mg}, 0.250 \mathrm{mmol}, 1.0$ equiv) and (Z)-2-cyano-1phenylpent-1-en-1-yl trifluoromethanesulfonate $2 \mathbf{a}(79.8 \mathrm{mg}, 0.250 \mathrm{mmol}, 1.0$ equiv, dr>20:1) were then added successively. The flask fitted with a balloon of carbon monoxide gas and partially evacuated followed by purging with carbon monoxide. This process was repeated three times. The reaction mixture was stirred at $60^{\circ} \mathrm{C}$ for 18 hours. After the reaction was complete, the reaction mixture was diluted with $\mathrm{Et}_{2} \mathrm{O}(10 \mathrm{~mL})$ and filtrated through a small pad of silica gel. The solvent was removed under reduced pressure with the aid of a rotary evaporator and the crude residue was purified by a silica gel column chromatography (Pentane:EtOAc $=100: 1)$ to give pure product $\mathbf{8}$ as a colorless oil in 92\% yield (52.8 mg, dr>20:1). $\mathbf{R}_{\mathrm{f}}=0.5$ (Pentane:EtOAc $\left.=10: 1\right) ;{ }^{1} \mathbf{H}$ NMR (300 MHz, $\left.\mathrm{CDCl}_{3}, 300 \mathrm{~K}\right): \delta(\mathrm{ppm})=7.38-7.32(\mathrm{~m}, 3 \mathrm{H}), 7.13-7.09(\mathrm{~m}, 2 \mathrm{H})$, $3.73(\mathrm{~s}, 3 \mathrm{H}), 2.14(\mathrm{t}, J=7.5 \mathrm{~Hz}, 2 \mathrm{H}), 1.56\left(\mathrm{tq}, J^{1}=7.5 \mathrm{~Hz}, J^{2}=7.5 \mathrm{~Hz}, 2 \mathrm{H}\right), 0.79(\mathrm{t}, J$ $=7.4 \mathrm{~Hz}, 3 \mathrm{H}) ;{ }^{13} \mathrm{C}$ NMR $\left(75 \mathrm{MHz}, \mathrm{CDCl}_{3}, 300 \mathrm{~K}\right): \delta(\mathrm{ppm})=165.4(\mathrm{C}), 146.4(\mathrm{C})$, $133.2(\mathrm{C}), 129.0(\mathrm{CH}), 128.5(\mathrm{CH}), 128.3(\mathrm{CH}), 121.8(\mathrm{C}), 117.1(\mathrm{C}), 52.8\left(\mathrm{CH}_{3}\right), 34.0$ $\left(\mathrm{CH}_{2}\right), 21.1\left(\mathrm{CH}_{2}\right), 13.2\left(\mathrm{CH}_{3}\right)$; HRMS (ESI) $\mathrm{m} / z=252.0995$, calcd. for $\mathrm{C}_{14} \mathrm{H}_{15} \mathrm{NO}_{2} \mathrm{Na}$ $[\mathrm{M}+\mathrm{Na}]^{+}$, found: 252.0996; IR (neat, $\mathrm{cm}^{-1}$ ): 2964w, 2875w, 2362w, 2218w, 2029w, $1972 w, 1728 s, 1615 w, 1436 w, 1298 m, 1224 s, 1085 w, 1015 w, 846 w, 791 w, 752 w, 701 m$.

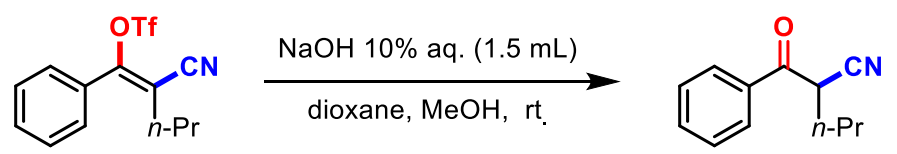<smiles>CC(F)(F)C(C#N)C(=O)c1ccccc1</smiles>

2-Benzoylpentanenitrile (9): A flame-dried Schlenk-flask equipped with a magnetic stir bar was charged with (Z)-2-cyano-1-phenylpent-1en-1-yl trifluoromethanesulfonate $\mathbf{2 a}(63.9 \mathrm{mg}, 0.200 \mathrm{mmol}, 1.0$ equiv, 
dr $>20: 1$ ), sealed with a septum, and degassed by alternating vacuum evacuation and argon backfilling (three times) before dioxane $(3 \mathrm{~mL})$ and methanol $(1 \mathrm{~mL})$ was added. Sodium hydroxide (10\% aqueous solution) $(1.5 \mathrm{~mL})$ was added dropwise to the resulting solution. The reaction mixture was stirred at room temperature for 10 hours. After the reaction was complete, the reaction mixture was quenched with $10 \mathrm{~mL}$ of $\mathrm{NH}_{4} \mathrm{Cl}$ (saturated aq. solution) and extracted with $\mathrm{Et}_{2} \mathrm{O}(3 \times 10 \mathrm{~mL})$. After drying over $\mathrm{Na}_{2} \mathrm{SO}_{4}$ and filtration, the solvent was removed under reduced pressure with the aid of a rotary evaporator to give pure product $\mathbf{9}$ as a yellow oil in $95 \%$ yield $(35.6 \mathrm{mg})$. $\mathbf{R}_{\mathrm{f}}=$ 0.35 (Pentane:EtOAc = 10:1); ${ }^{1} \mathbf{H}$ NMR $\left(300 \mathrm{MHz}, \mathrm{CDCl}_{3}, 300 \mathrm{~K}\right): \delta(\mathrm{ppm})=7.87-$ 7.90 (m, 2H), 7.57 (t, $J=7.4 \mathrm{~Hz}, 1 \mathrm{H}), 7.44$ (t, $J=7.6 \mathrm{~Hz}, 2 \mathrm{H}), 4.30$ (t, $J=7.1 \mathrm{~Hz}, 1 \mathrm{H})$, $1.90\left(\mathrm{dt}, J^{1}=7.8, J^{2}=7.2 \mathrm{~Hz}, 2 \mathrm{H}\right), 1.64-1.39(\mathrm{~m}, 2 \mathrm{H}), 0.92(\mathrm{t}, J=7.3 \mathrm{~Hz}, 3 \mathrm{H}) .{ }^{13} \mathrm{C}$ NMR (75 MHz, $\left.\mathrm{CDCl}_{3}, 300 \mathrm{~K}\right): \delta(\mathrm{ppm})=190.9(\mathrm{C}), 134.4(\mathrm{CH}), 134.0(\mathrm{C}), 129.0$ $(\mathrm{CH}), 128.7(\mathrm{CH}), 117.3(\mathrm{C}), 39.8(\mathrm{CH}), 31.8\left(\mathrm{CH}_{2}\right), 20.4\left(\mathrm{CH}_{2}\right), 13.4\left(\mathrm{CH}_{3}\right)$; HRMS (ESI) $m / z=210.0889$, calcd. for $\mathrm{C}_{12} \mathrm{H}_{13} \mathrm{NONa}[\mathrm{M}+\mathrm{Na}]^{+}$, found: 210.0894 ; IR (neat, $\left.\mathrm{cm}^{-1}\right): 3064 w, 2963 w, 2875 w, 2249 w, 1691 s, 1597 s, 1449 m, 1344 w, 1262 m, 1226 s$, $1090 w, 986 w, 918 w, 803 w, 780 w, 693 s$.
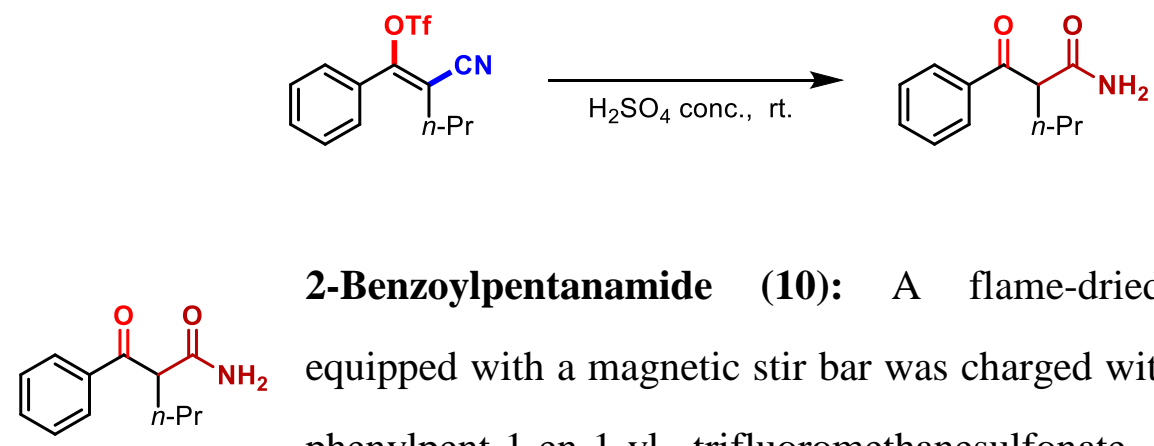

2-Benzoylpentanamide (10): A flame-dried Schlenk-flask equipped with a magnetic stir bar was charged with (Z)-2-cyano-1phenylpent-1-en-1-yl trifluoromethanesulfonate $\mathbf{2 a}(127.7 \mathrm{mg}$, 0.4000 mmol, 1.0 equiv, dr>20:1), sealed with a septum, and degassed by alternating vacuum evacuation and argon backfilling (three times) before concentrated sulfuric acid $(1 \mathrm{~mL})$ was added at $0{ }^{\circ} \mathrm{C}$. The reaction mixture was allowed to warm to room temperature and stirring at room temperature was continued for 24 hours. After the reaction was complete, the reaction mixture was then poured into ice water, basified with $28 \%$ ammonium hydroxide solution and extracted with EtOAc. The organic phase 
was washed with brine, dried over $\mathrm{Na}_{2} \mathrm{SO}_{4}$. After drying over $\mathrm{Na}_{2} \mathrm{SO}_{4}$ and filtration, the solvent was removed under reduced pressure with the aid of a rotary evaporator to give pure product $\mathbf{1 0}$ as a white solid in $96 \%$ yield $(78.4 \mathrm{mg}) . \mathbf{R}_{\mathrm{f}}=0.2($ Pentane:EtOAc $=$ 2:1); MP: $158{ }^{\circ} \mathrm{C} ;{ }^{1} \mathbf{H}$ NMR (300 MHz, $\left.\mathrm{CD}_{2} \mathrm{Cl}_{2}, 300 \mathrm{~K}\right): \delta(\mathrm{ppm})=7.98-7.95(\mathrm{~m}, 2 \mathrm{H})$, $7.59-7.54(\mathrm{~m}, 1 \mathrm{H}), 7.45(\mathrm{t}, J=7.5 \mathrm{~Hz}, 2 \mathrm{H}), 6.30(\mathrm{~s}, 1 \mathrm{H}), 5.53(\mathrm{~s}, 1 \mathrm{H}), 4.27(\mathrm{t}, J=7.2$ $\mathrm{Hz}, 1 \mathrm{H}), 1.99-1.79(\mathrm{~m}, 2 \mathrm{H}), 1.37-1.29(\mathrm{~m}, 2 \mathrm{H}), 0.91-0.85(\mathrm{~m}, 3 \mathrm{H}) ;{ }^{13} \mathbf{C}$ NMR $(75$ $\left.\mathrm{MHz}, \mathrm{CD}_{2} \mathrm{Cl}_{2}, 300 \mathrm{~K}\right): \delta(\mathrm{ppm})=199.6(\mathrm{C}), 171.6(\mathrm{C}), 137.2(\mathrm{C}), 134.4(\mathrm{CH}), 129.4$ (CH), $129.1(\mathrm{CH}), 56.2(\mathrm{CH}), 34.7\left(\mathrm{CH}_{2}\right), 21.5\left(\mathrm{CH}_{2}\right), 14.2\left(\mathrm{CH}_{3}\right)$; HRMS (ESI) $\mathrm{m} / \mathrm{z}=$ 228.0995, calcd. for $\mathrm{C}_{12} \mathrm{H}_{15} \mathrm{NO}_{2} \mathrm{Na}[\mathrm{M}+\mathrm{Na}]^{+}$, found: 228.1007 ; IR (neat, $\mathrm{cm}^{-1}$ ): $3405 \mathrm{br}$, $3178 b r, 2957 w, 2873 w, 2195 w, 1681 s, 1650 s, 1449 w, 1394 w, 1337 w, 1275 w, 1209 w$, $1122 w, 983 w, 821 w, 769 w, 704 w, 628 w$.
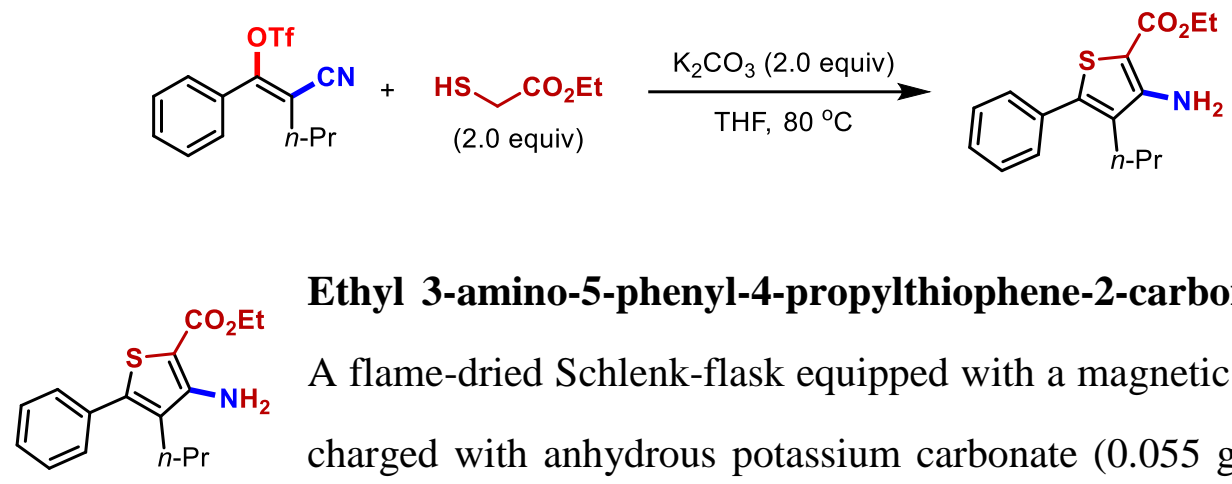

Ethyl 3-amino-5-phenyl-4-propylthiophene-2-carboxylate (11): A flame-dried Schlenk-flask equipped with a magnetic stir bar was charged with anhydrous potassium carbonate $(0.055 \mathrm{~g}, 0.4 \mathrm{mmol}$, 2.0 equiv), sealed with a septum, and degassed by alternating vacuum evacuation and argon backfilling (three times) before the freshly distilled THF (2 mL) was added. (Z)2-cyano-1-phenylpent-1-en-1-yl trifluoromethanesulfonate 2a $(63.9 \mathrm{mg}, 0.200 \mathrm{mmol}$, 1.0 equiv, dr>20:1) was added to the resulting suspension subsequently. The reaction mixture was stirred at $80^{\circ} \mathrm{C}$ for 5 hours. After the reaction was complete, the solvent was removed under reduced pressure with the aid of a rotary evaporator and the crude residue was purified by a silica gel column chromatography (Pentane:EtOAc $=100: 1)$ to give pure product $\mathbf{1 1}$ as a colorless oil in $48 \%$ yield $(27.6 \mathrm{mg}) . \mathbf{R}_{\mathrm{f}}=0.25$ $($ Pentane:EtOAc $=10: 1) ;{ }^{1} \mathbf{H}$ NMR $\left(300 \mathrm{MHz}, \mathrm{CDCl}_{3}, 300 \mathrm{~K}\right): \delta(\mathrm{ppm})=7.35-7.23$ $(\mathrm{m}, 5 \mathrm{H}), 4.87(\mathrm{br}, 2 \mathrm{H}) 4.24(\mathrm{q}, J=7.1 \mathrm{~Hz}, 2 \mathrm{H}), 2.39(\mathrm{t}, J=8.1 \mathrm{~Hz}, 2 \mathrm{H}), 1.48\left(\mathrm{tq}, J^{1}=\right.$ $\left.7.8 \mathrm{~Hz}, J^{2}=7.5 \mathrm{~Hz}, 2 \mathrm{H}\right), 1.28(\mathrm{t}, J=7.1 \mathrm{~Hz}, 3 \mathrm{H}), 0.82(\mathrm{t}, J=7.3 \mathrm{~Hz}, 3 \mathrm{H}) ;{ }^{13} \mathbf{C} \mathbf{N M R}$ $\left(75 \mathrm{MHz}, \mathrm{CDCl}_{3}, 300 \mathrm{~K}\right): \delta(\mathrm{ppm})=164.9$ (C), 153.0 (C), 144.3 (C), 134.3 (C), 129.1 
$(\mathrm{CH}), 128.6(\mathrm{CH}), 128.5(\mathrm{C}), 128.3(\mathrm{CH}), 100.3(\mathrm{C}), 60.0\left(\mathrm{CH}_{2}\right), 28.2\left(\mathrm{CH}_{2}\right), 22.3$ $\left(\mathrm{CH}_{2}\right), 14.6\left(\mathrm{CH}_{3}\right), 14.1\left(\mathrm{CH}_{3}\right) ;{ }^{19} \mathbf{F} \mathbf{N M R}\left(282 \mathrm{MHz}, \mathrm{CDCl}_{3}, 300 \mathrm{~K}\right): \delta(\mathrm{ppm})=-73.5$ (s, 3F); HRMS (ESI) $m / z=312.1029$, calcd. for $\mathrm{C}_{16} \mathrm{H}_{19} \mathrm{NO}_{2} \mathrm{SNa}[\mathrm{M}+\mathrm{Na}]^{+}$, found: 312.1033; IR (neat, $\mathrm{cm}^{-1}$ ): 3479w, 3362w, 2961w, 2871w, 2253w, 2157w, 1669s, 1599s, $1549 w, 1469 m, 1369 w, 1306 s, 1234 m, 1172 w, 1126 m, 1084 m, 1028 w, 763 m, 698 m$, $616 w$. 
5. Spectra

Spectra of alkynes 3

${ }^{1}$ H NMR Spectrum of 1-methyl-4-(pent-1-yn-1-yl)benzene $3 \mathrm{~b}$

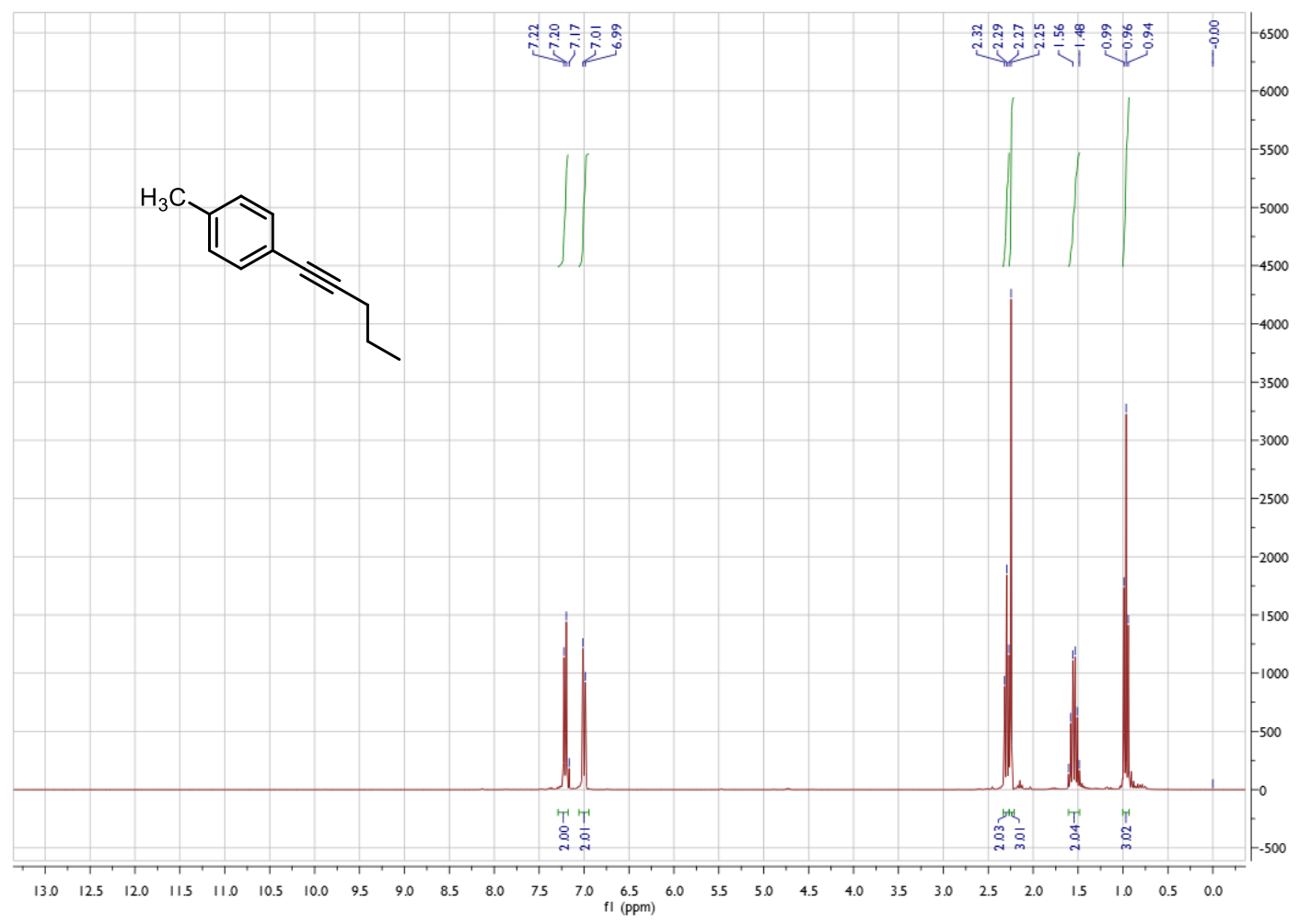

${ }^{13}$ C NMR Spectrum of 1-methyl-4-(pent-1-yn-1-yl)benzene Bb

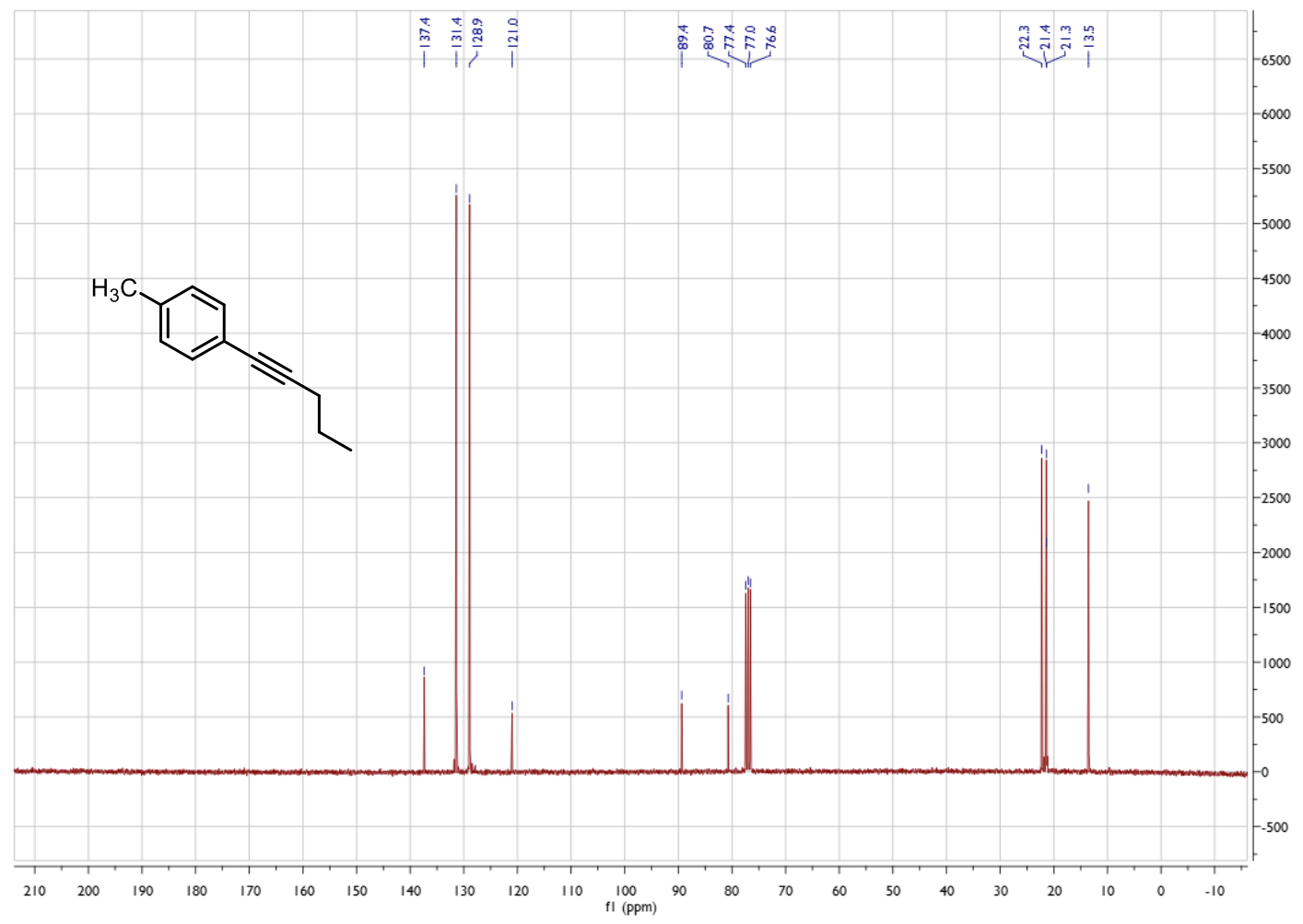


${ }^{1}$ H NMR Spectrum of 1-chloro-4-(pent-1-yn-1-yl)benzene Bc

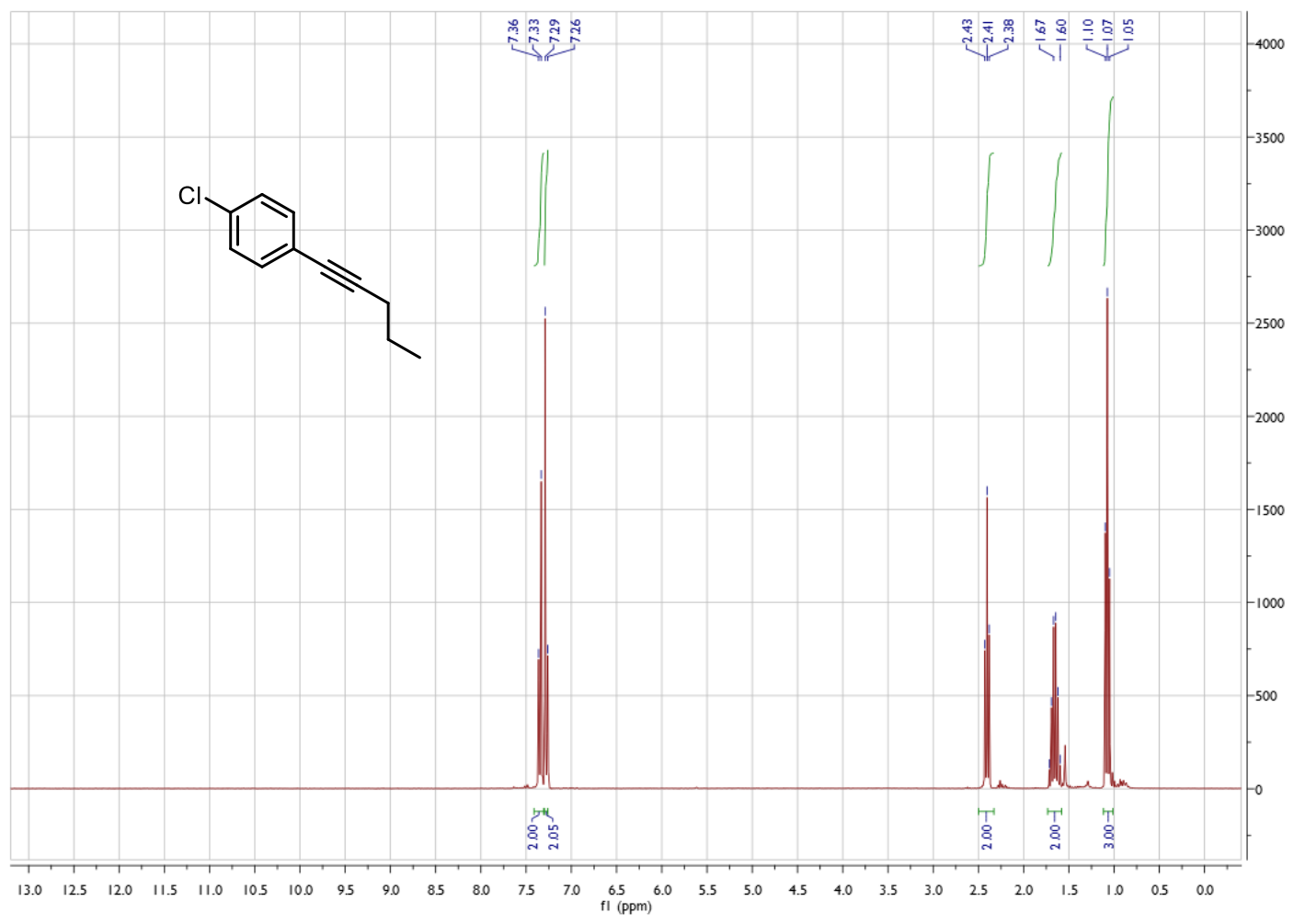

${ }^{13}$ C NMR Spectrum of 1-chloro-4-(pent-1-yn-1-yl)benzene Bc

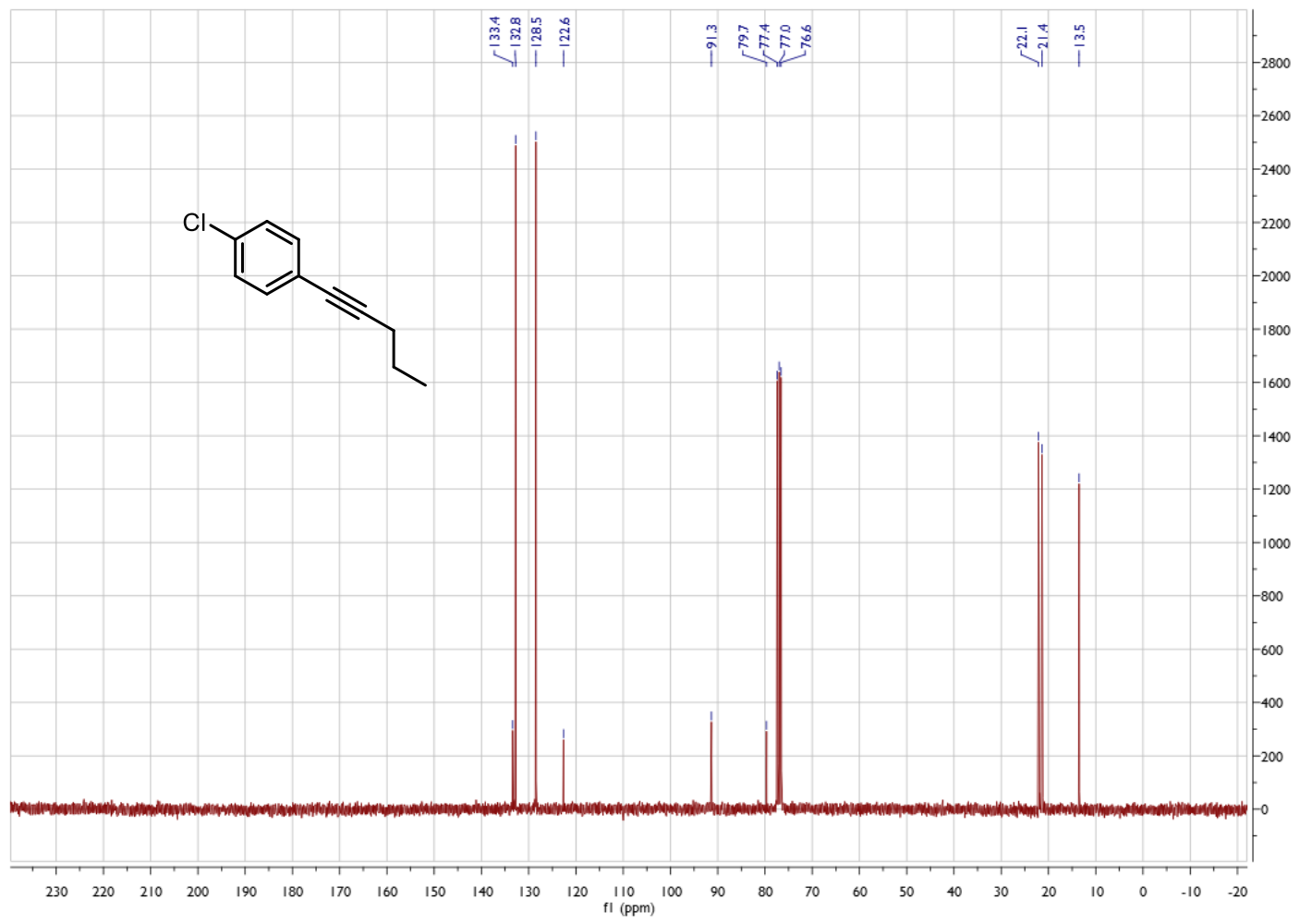


${ }^{1}$ H NMR Spectrum of 1-Chloro-2-methyl-4-(pent-1-yn-1-yl)benzene Bd

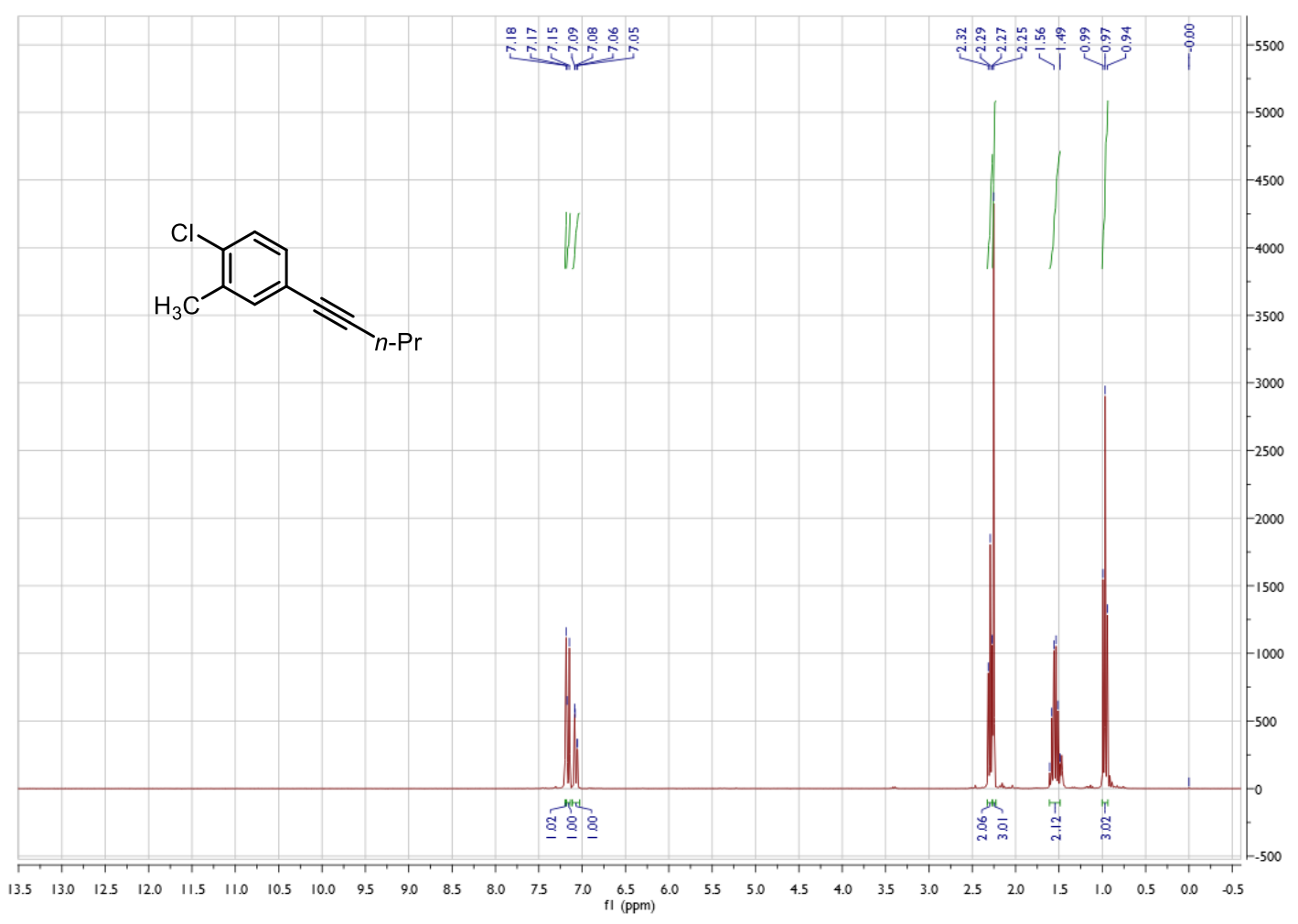

${ }^{13}$ C NMR Spectrum of 1-Chloro-2-methyl-4-(pent-1-yn-1-yl)benzene Bd

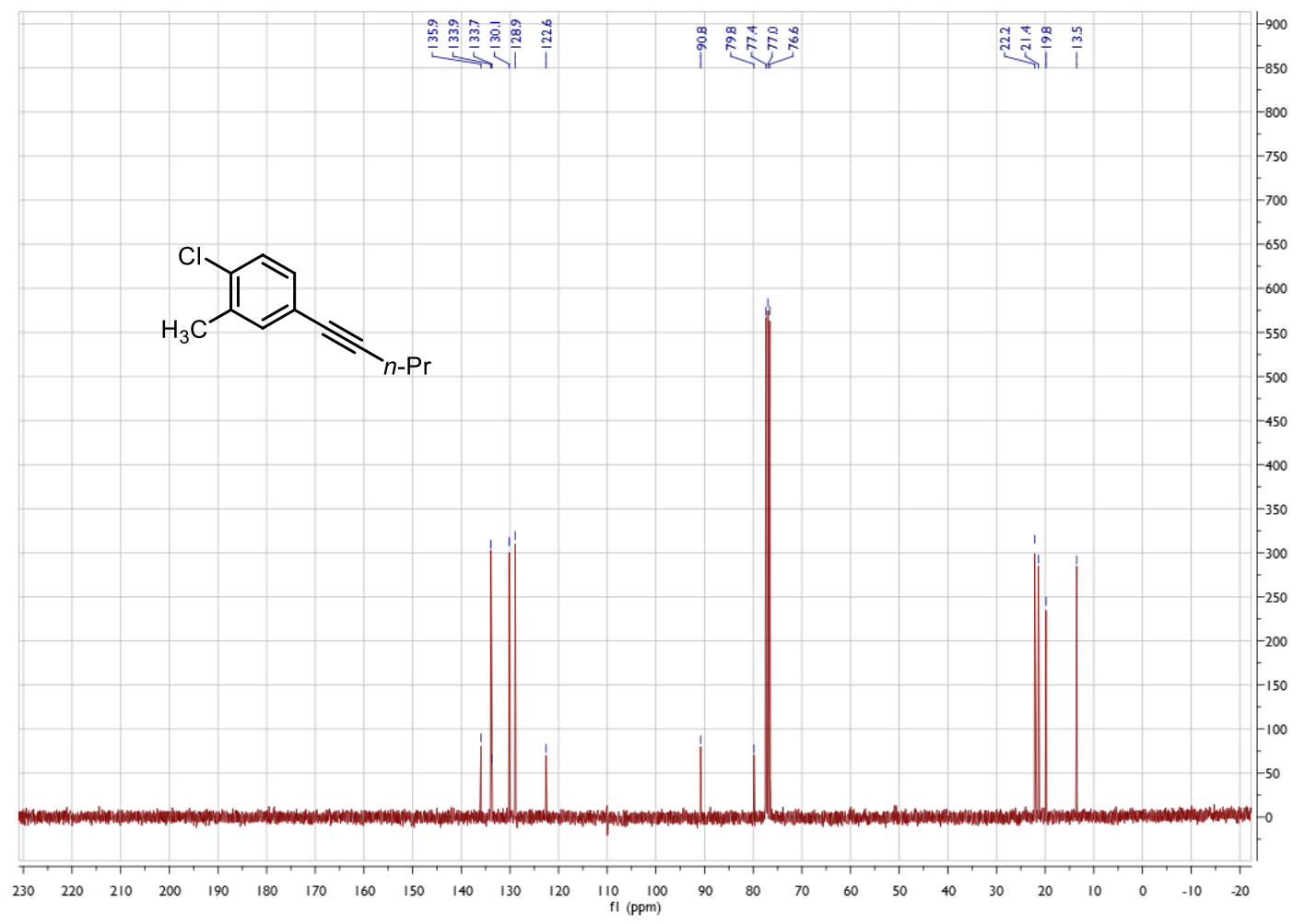


${ }^{1}$ H NMR Spectrum of 1-bromo-4-(pent-1-yn-1-yl)benzene $3 \mathrm{e}$

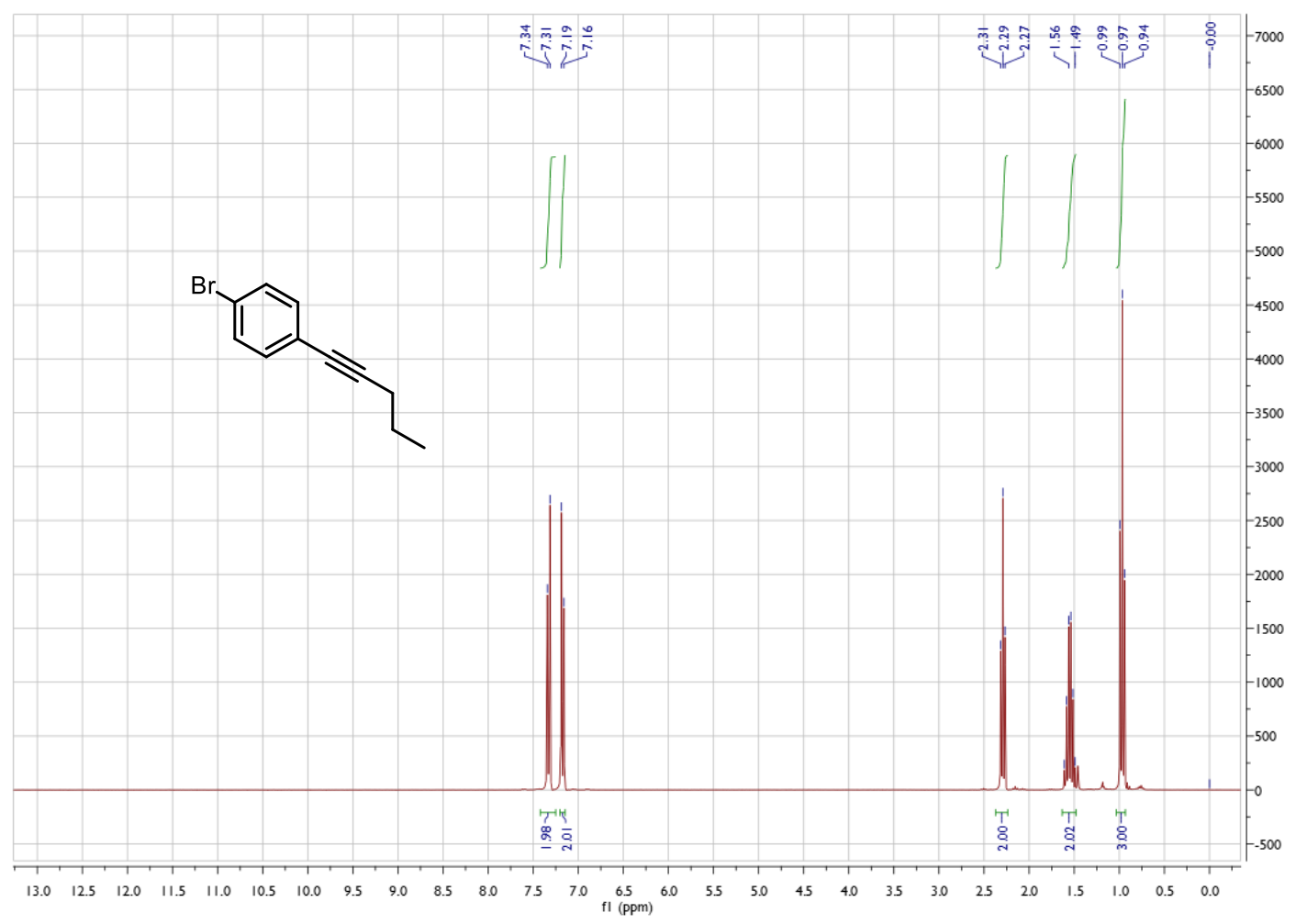

${ }^{13}$ C NMR Spectrum of 1-bromo-4-(pent-1-yn-1-yl)benzene $3 \mathrm{e}$

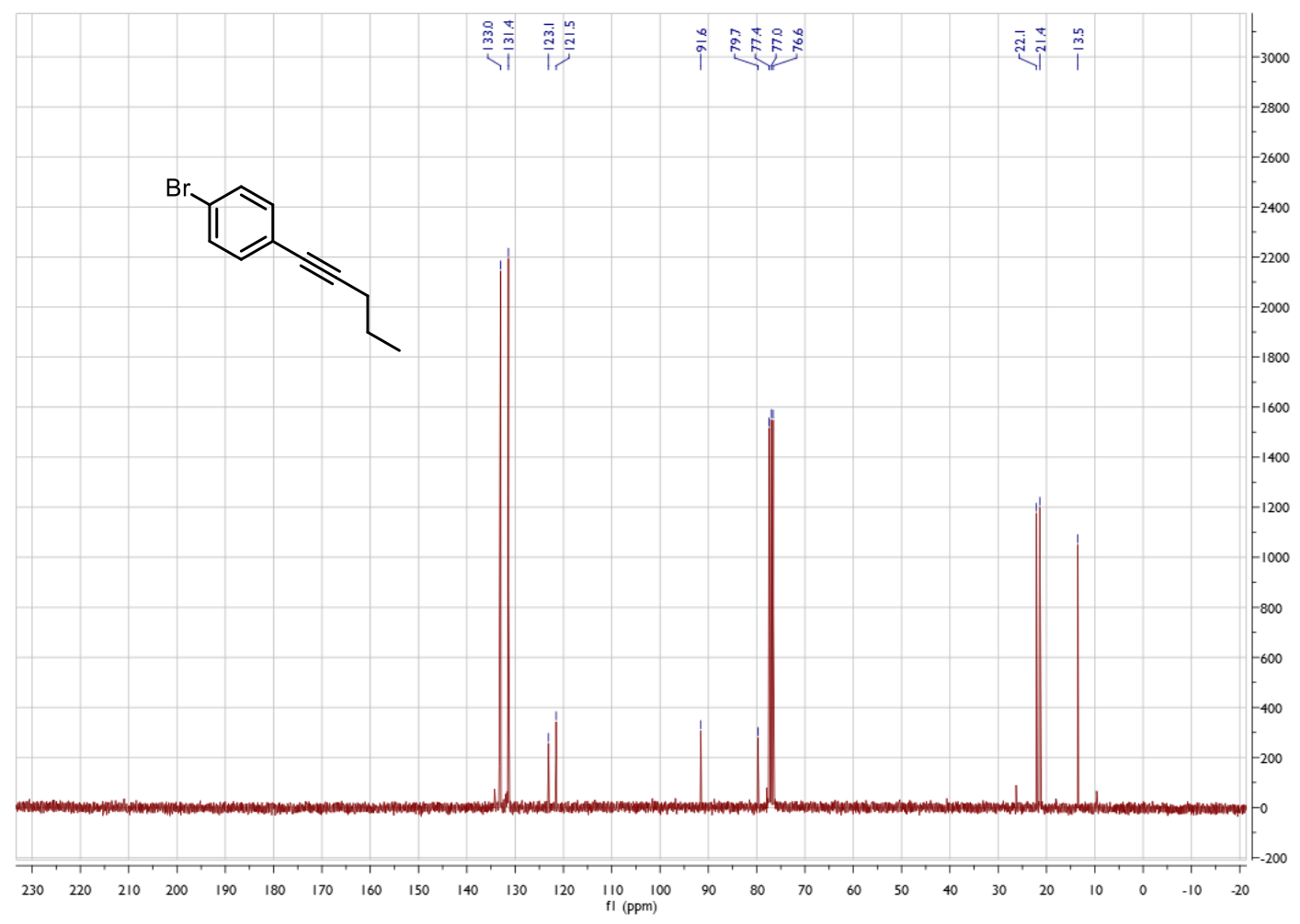


${ }^{1}$ H NMR Spectrum of 1-fluoro-4-(pent-1-yn-1-yl)benzene $3 f$

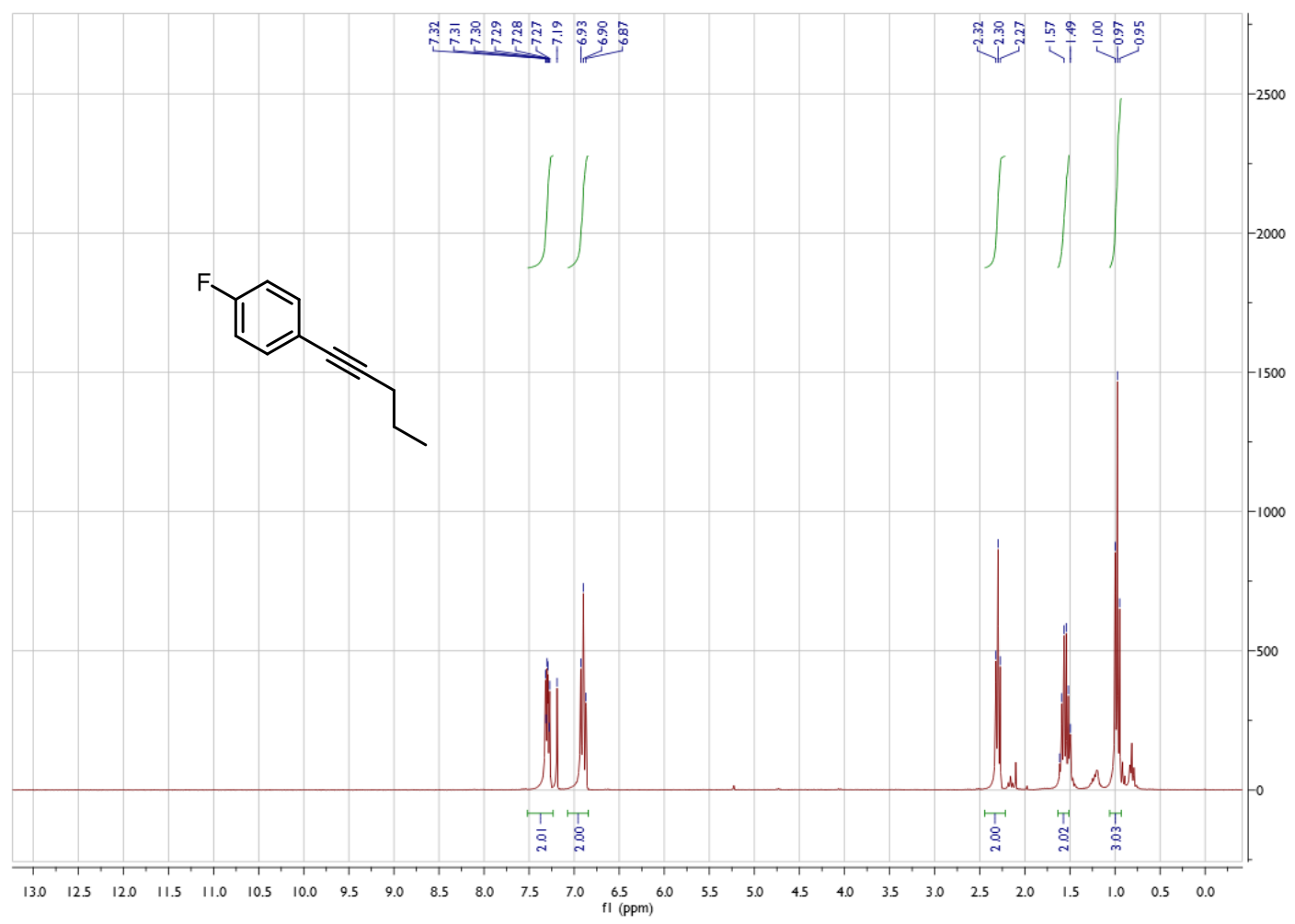

${ }^{13}$ C NMR Spectrum of 1-fluoro-4-(pent-1-yn-1-yl)benzene $3 f$

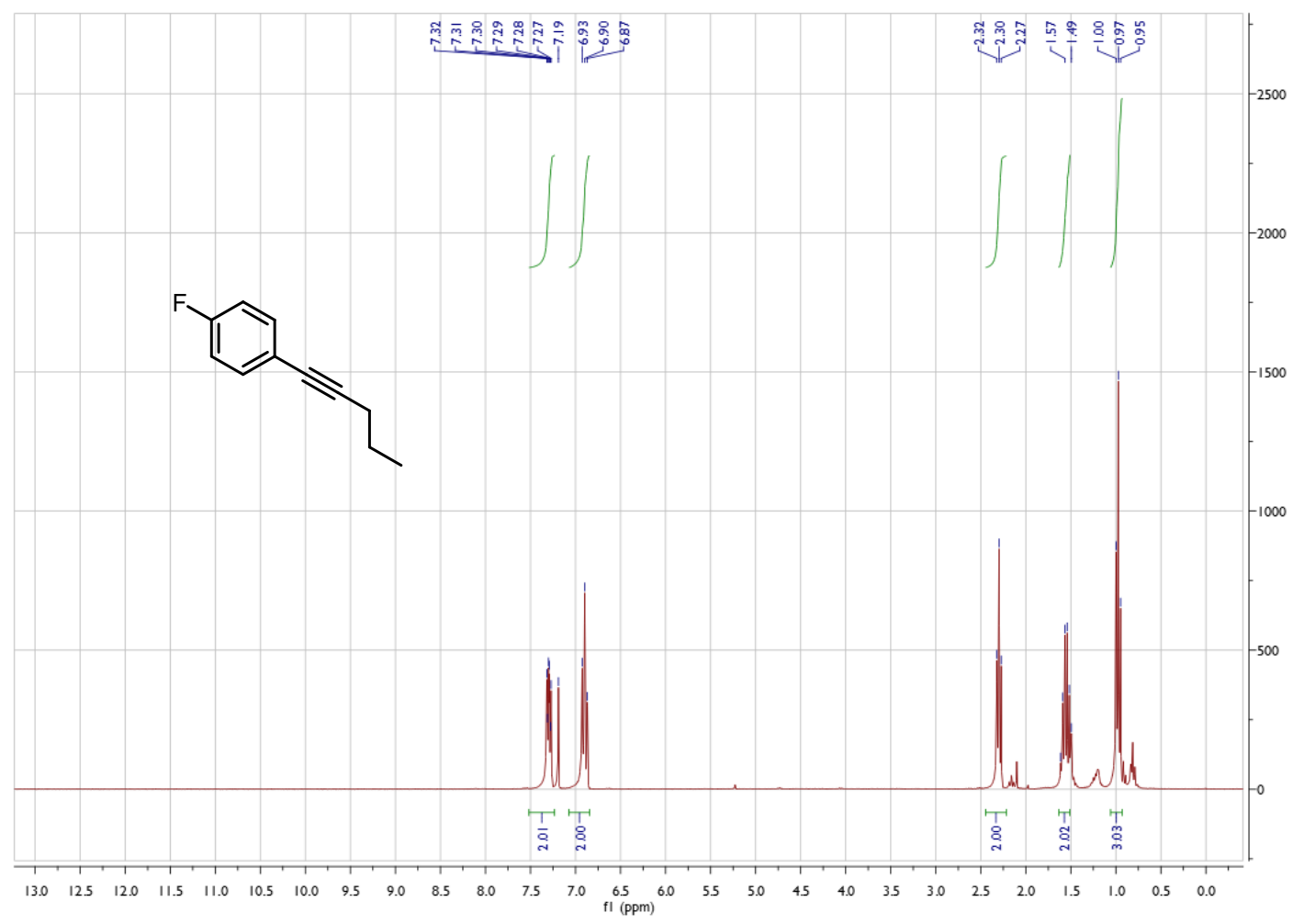


${ }^{19}$ F NMR Spectrum of 1-fluoro-4-(pent-1-yn-1-yl)benzene $3 f$

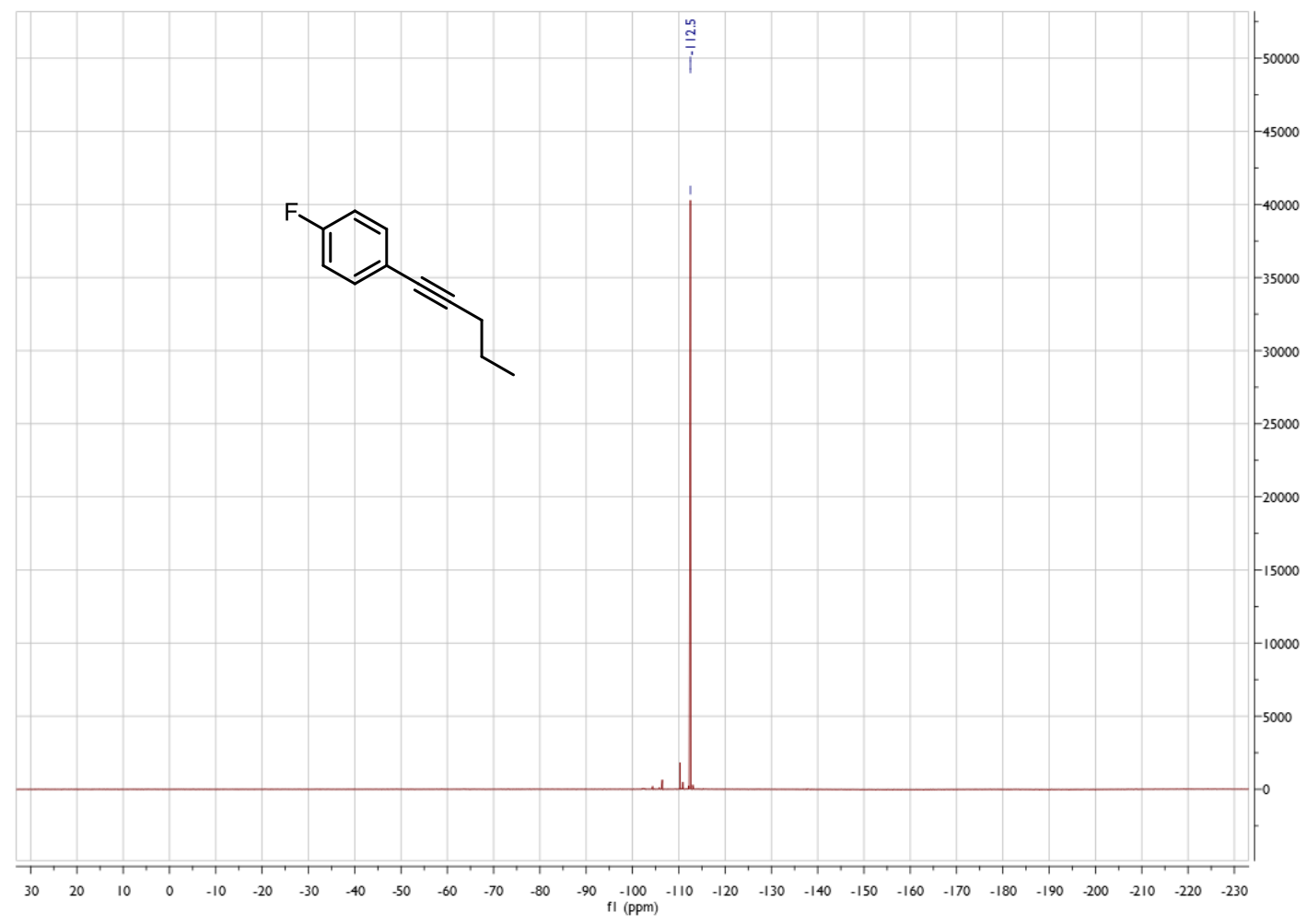


${ }^{1}$ H NMR Spectrum of 4-(pent-1-yn-1-yl)phenyl acetate $3 \mathrm{~g}$

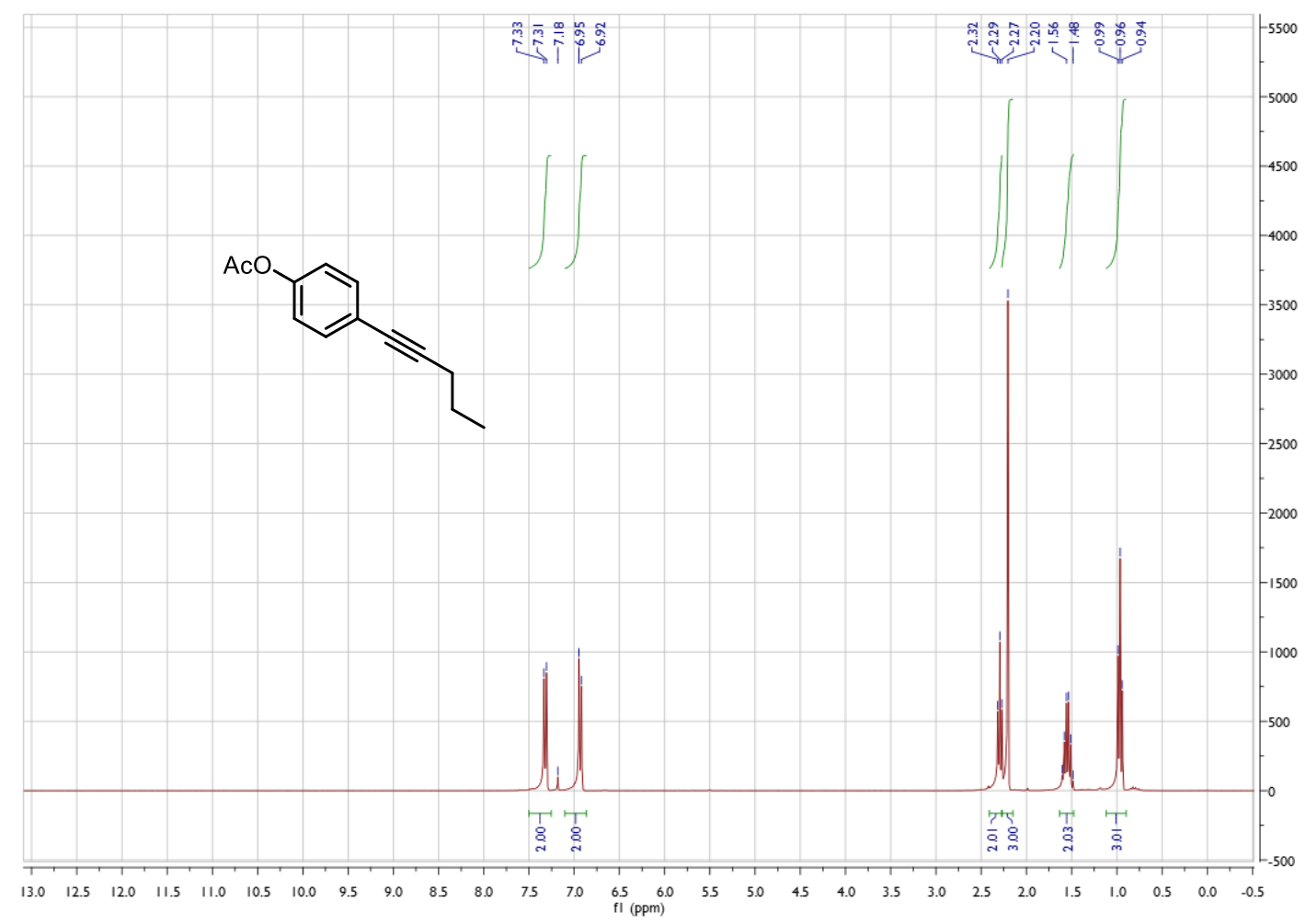

${ }^{13}$ C NMR Spectrum of 4-(pent-1-yn-1-yl)phenyl acetate $3 g$

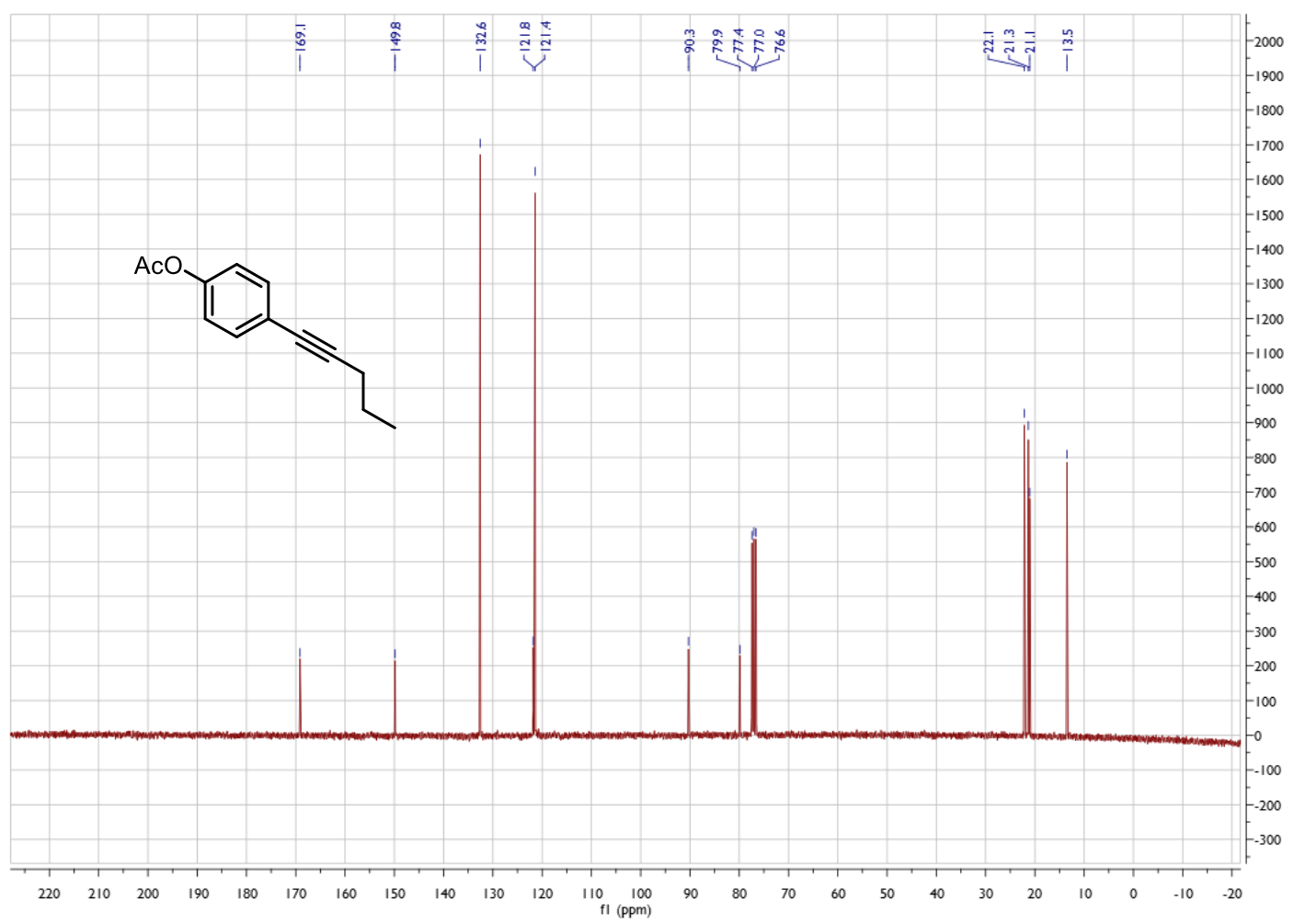


${ }^{1} \mathrm{H}$ NMR Spectrum of 2-(4-(pent-1-yn-1-yl)phenyl)isoindoline-1,3-dione $3 \mathrm{~h}$

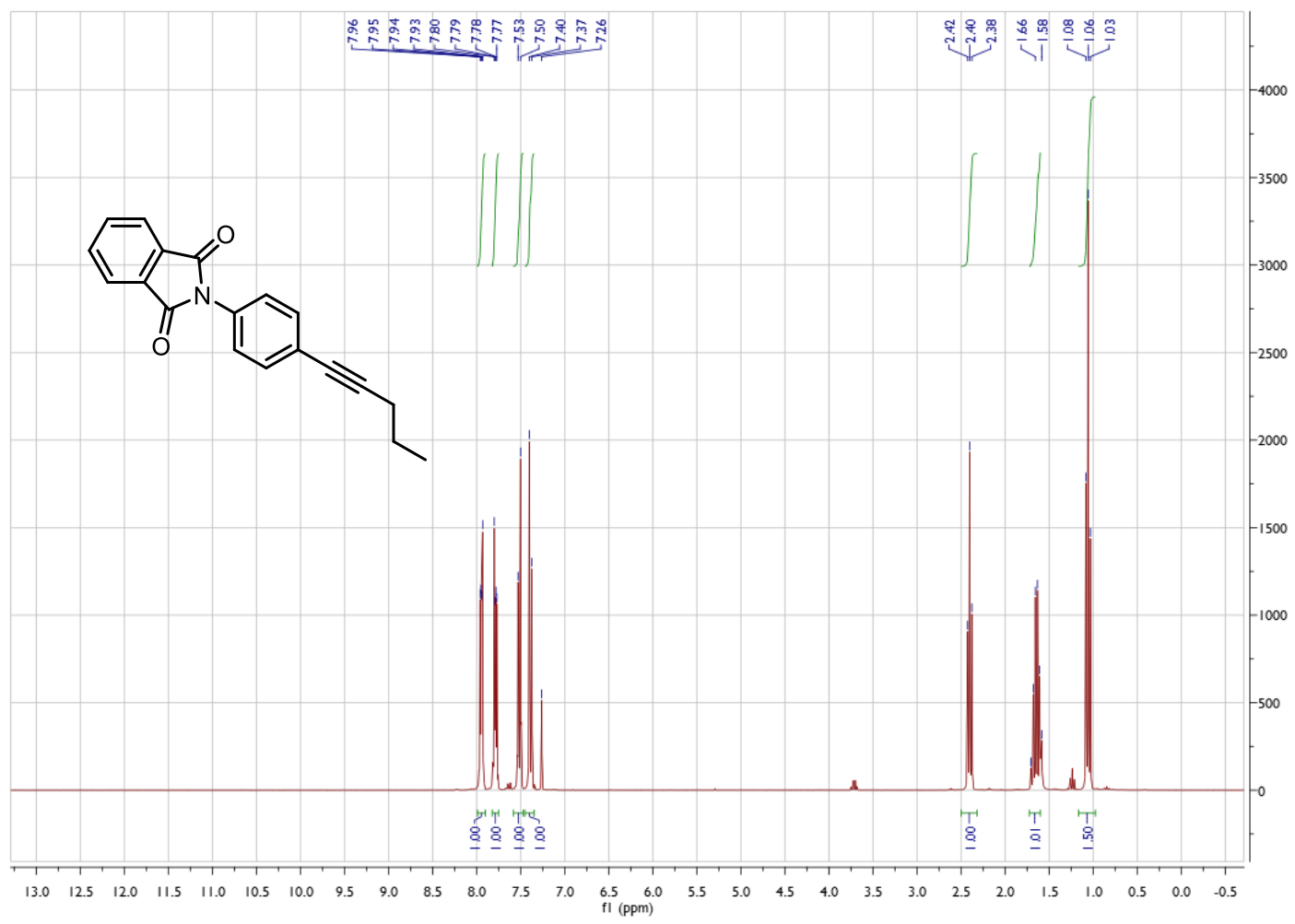

${ }^{13} \mathrm{C}$ NMR Spectrum of 2-(4-(pent-1-yn-1-yl)phenyl)isoindoline-1,3-dione $3 \mathrm{~h}$

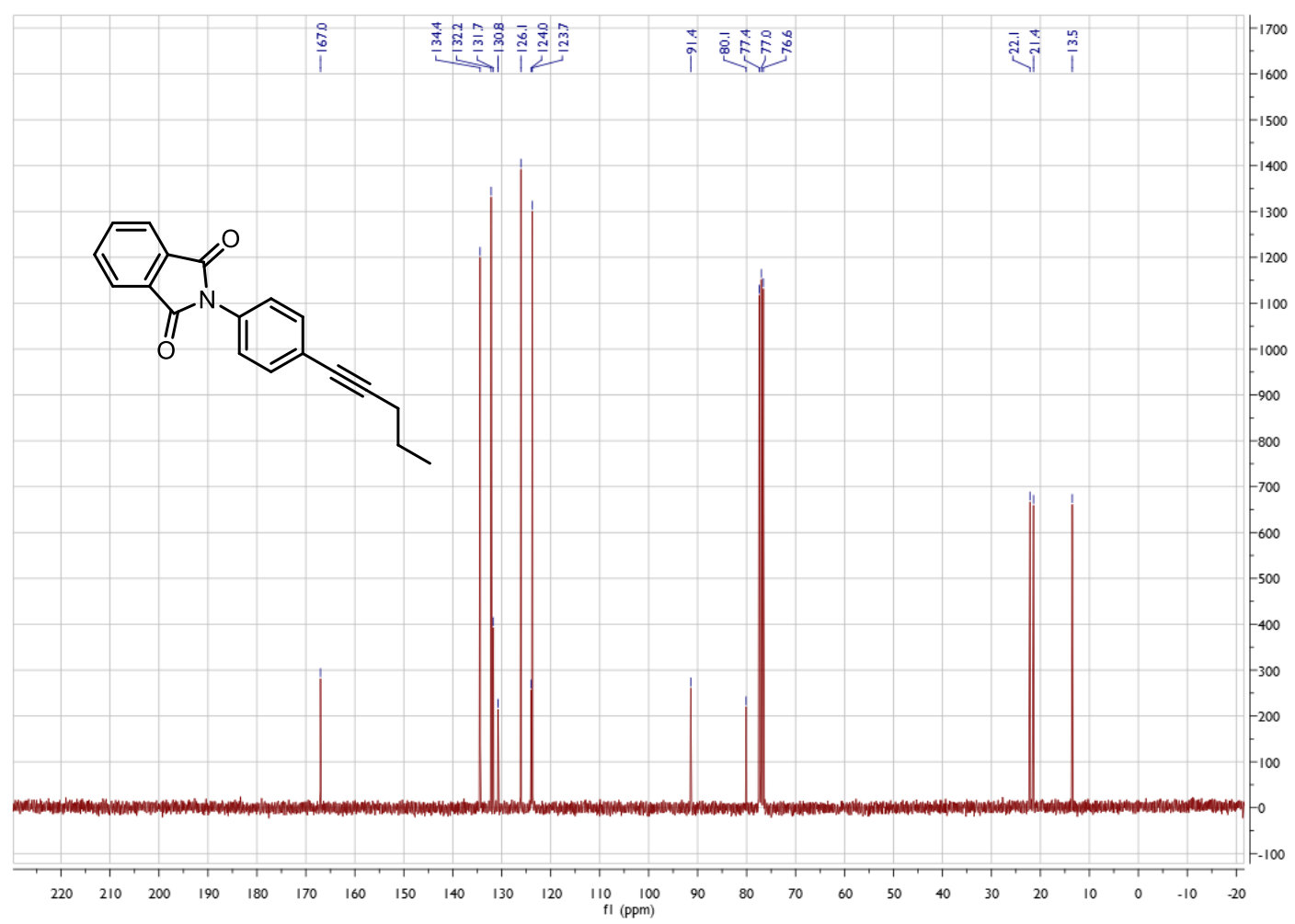


${ }^{1}$ H NMR Spectrum of (4-(pent-1-yn-1-yl)phenyl)(phenyl)methanone 3i

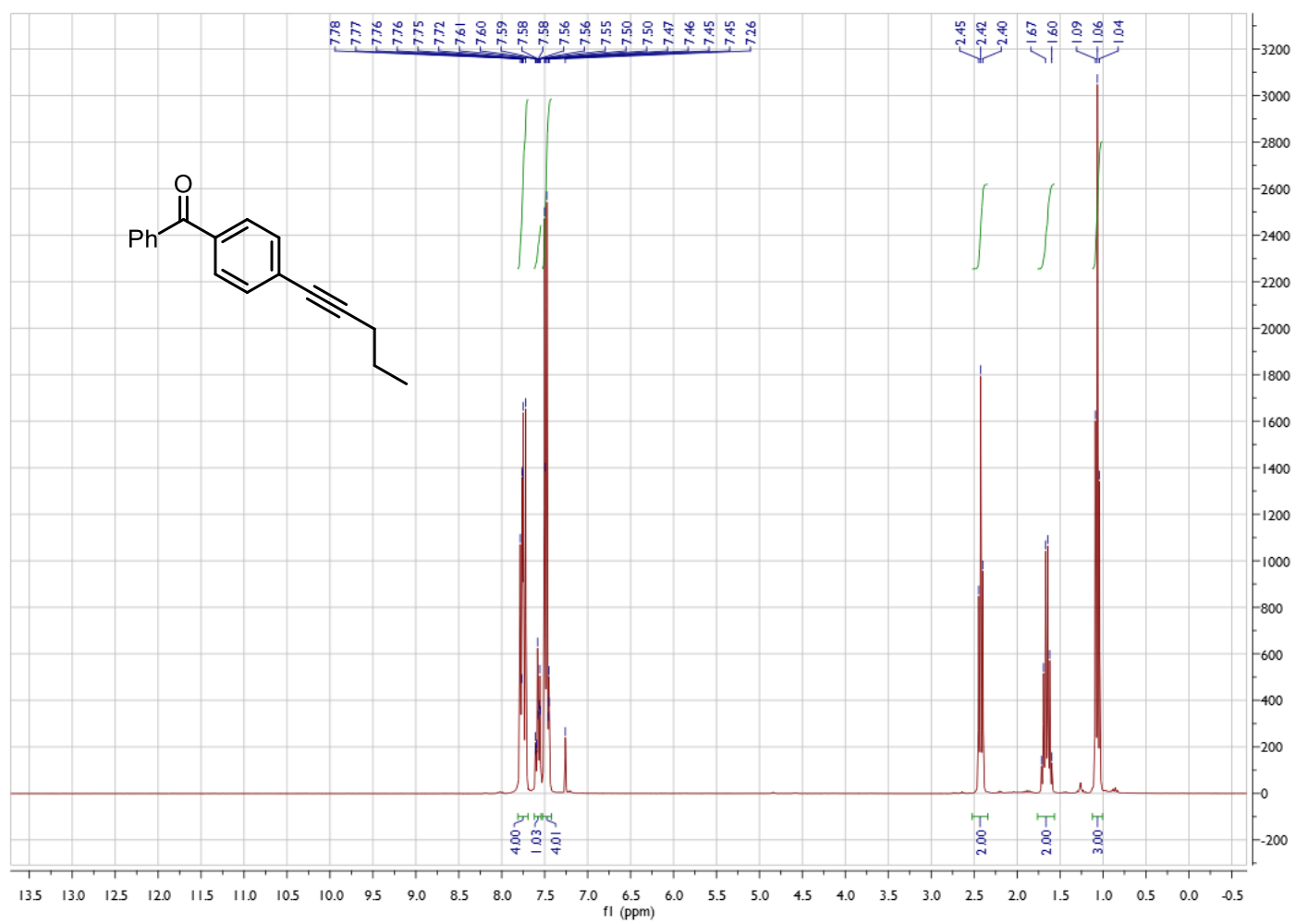

${ }^{13}$ C NMR Spectrum of (4-(pent-1-yn-1-yl)phenyl)(phenyl)methanone 3i

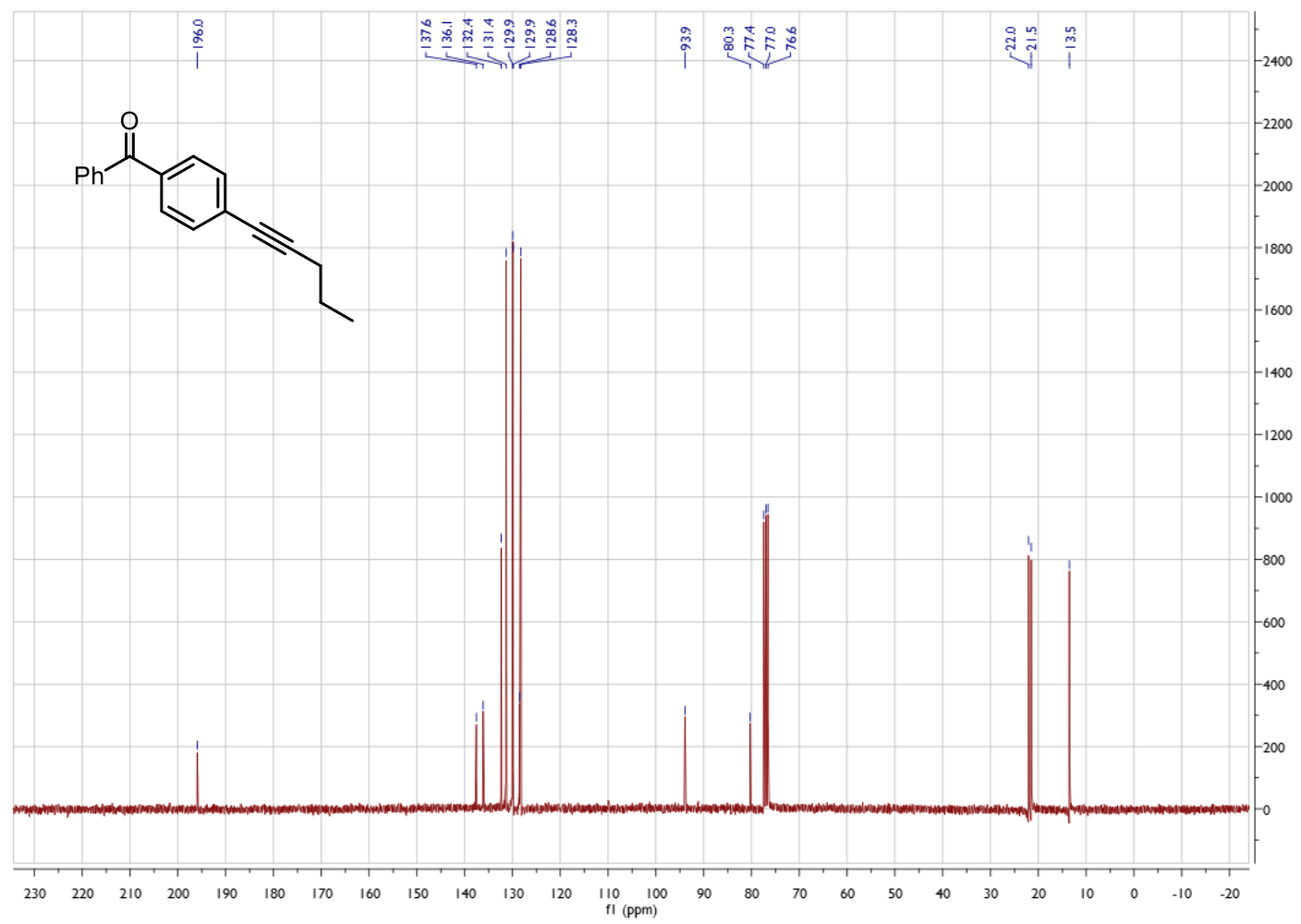


${ }^{1}$ H NMR Spectrum of 1-(pent-1-yn-1-yl)-4-(trifluoromethoxy)benzene 3j

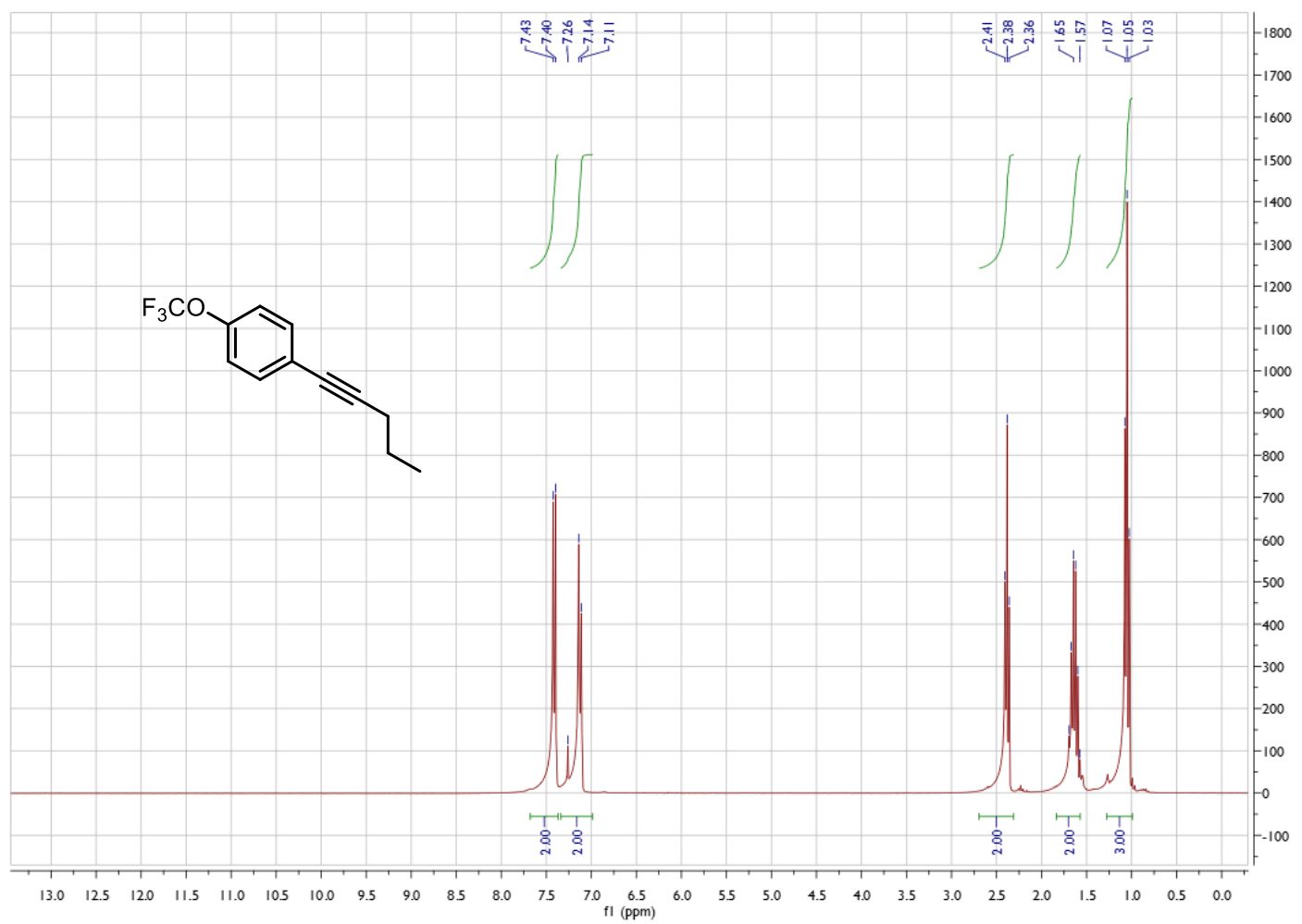

${ }^{1}$ H NMR Spectrum of 1-(pent-1-yn-1-yl)-4-(trifluoromethoxy)benzene 3j

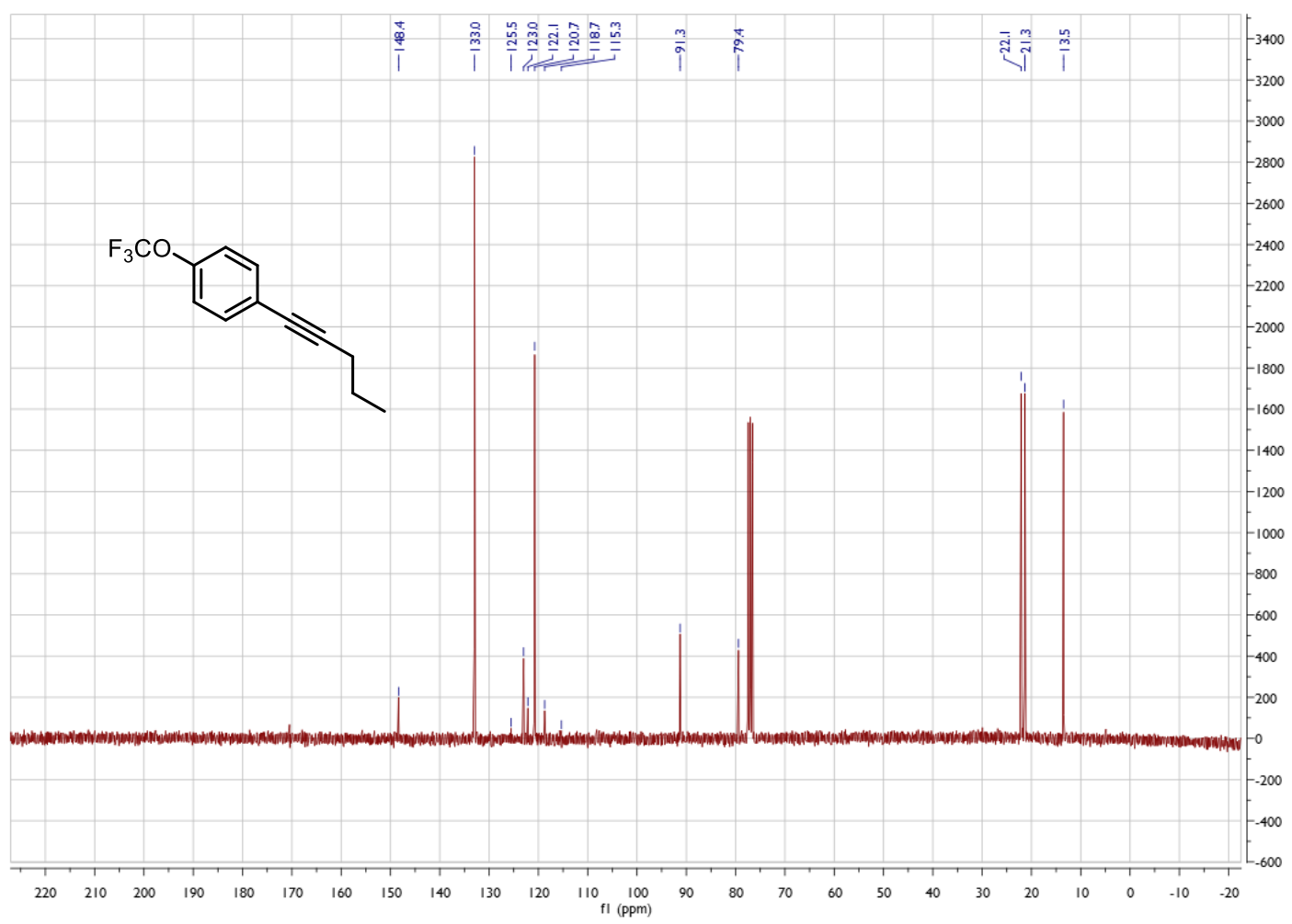


${ }^{1}$ H NMR Spectrum of (4-(pent-1-yn-1-yl)phenyl)(trifluoromethyl)sulfane 3k

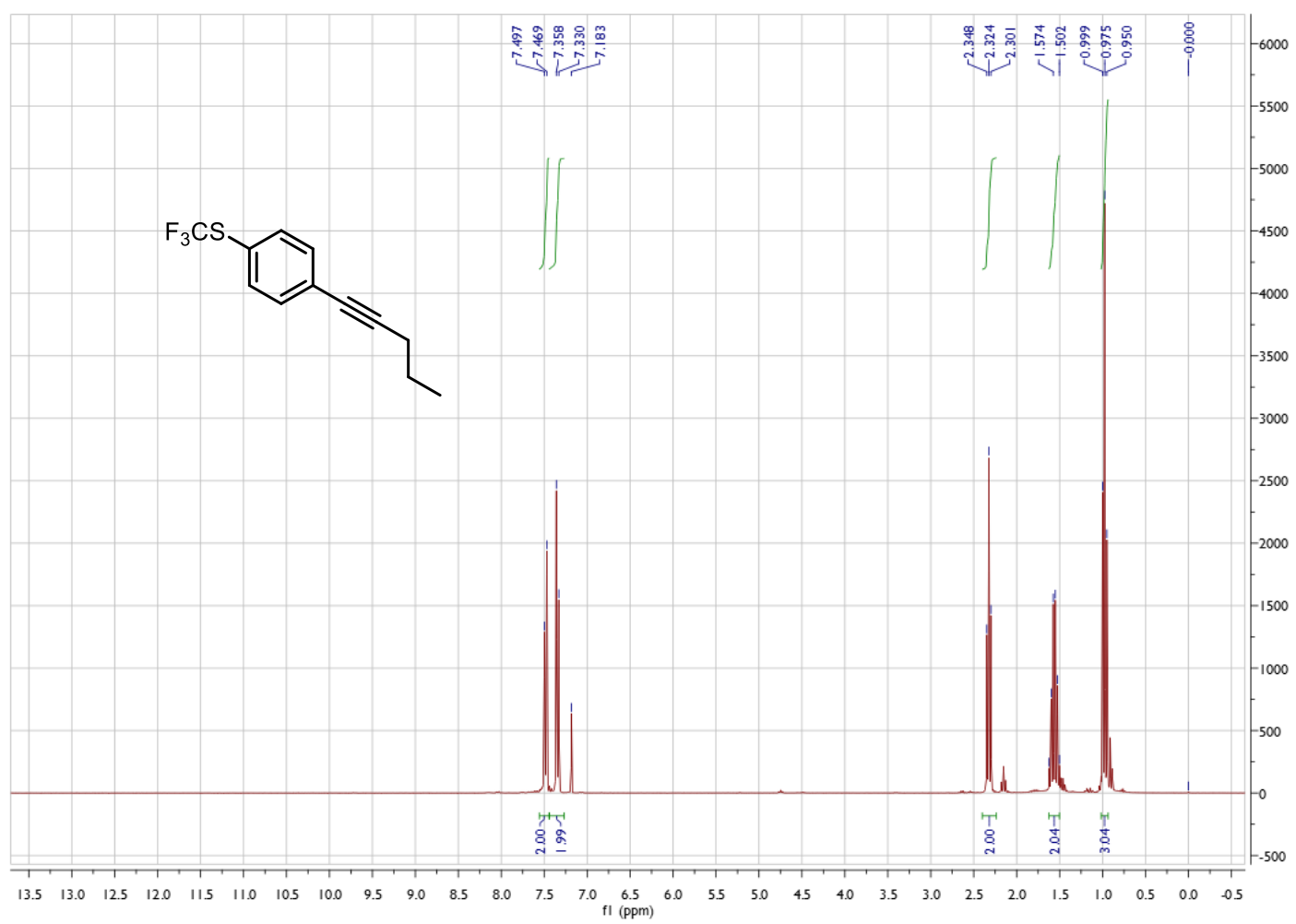

${ }^{13}$ C NMR Spectrum of (4-(pent-1-yn-1-yl)phenyl)(trifluoromethyl)sulfane $3 \mathrm{k}$

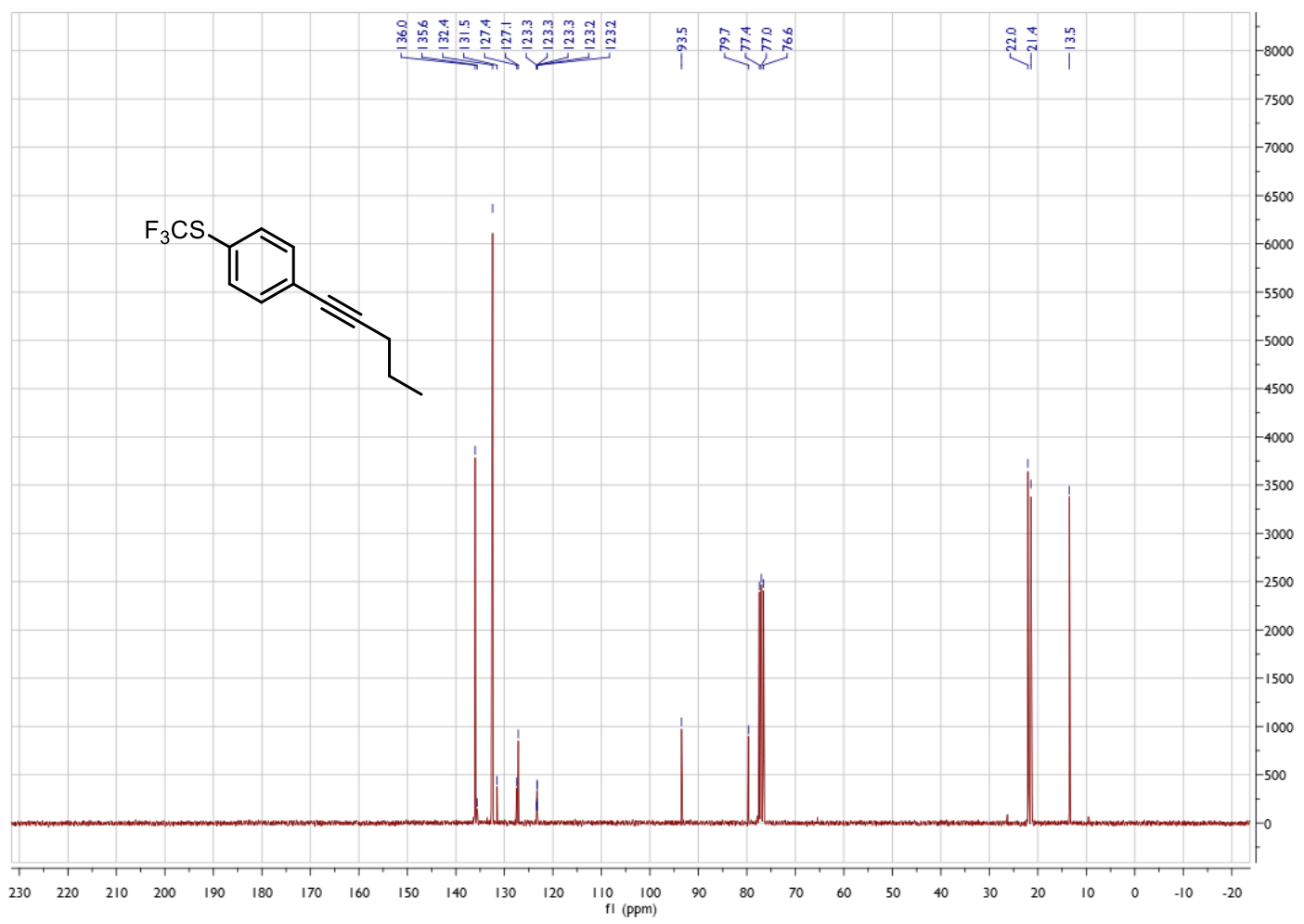


${ }^{1}$ H NMR Spectrum of ethyl 3-(pent-1-yn-1-yl)benzoate 31

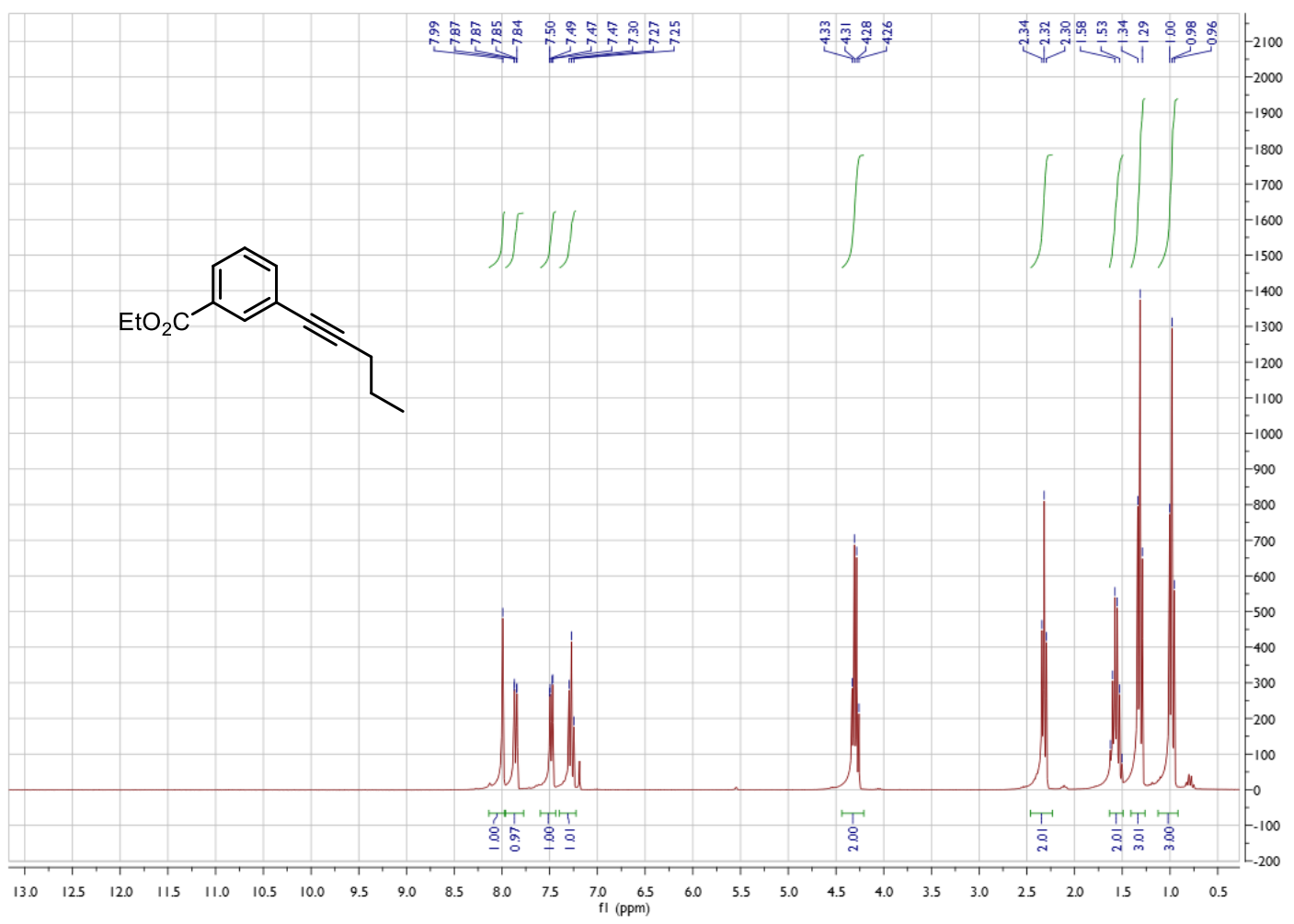

${ }^{13}$ C NMR Spectrum of ethyl 3-(pent-1-yn-1-yl)benzoate 3I

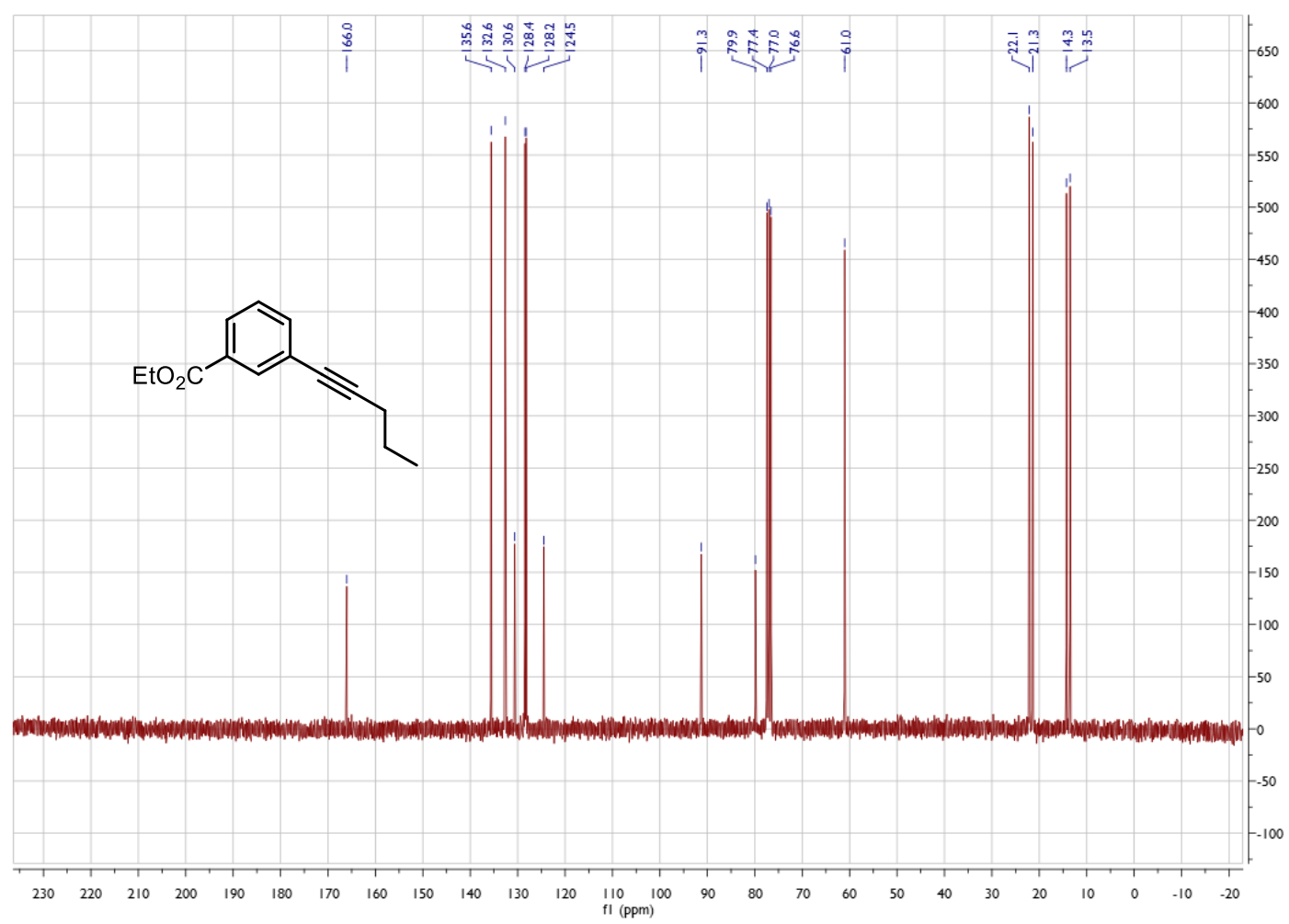


${ }^{1}$ H NMR Spectrum of 3-(Pent-1-yn-1-yl)benzaldehyde 3m

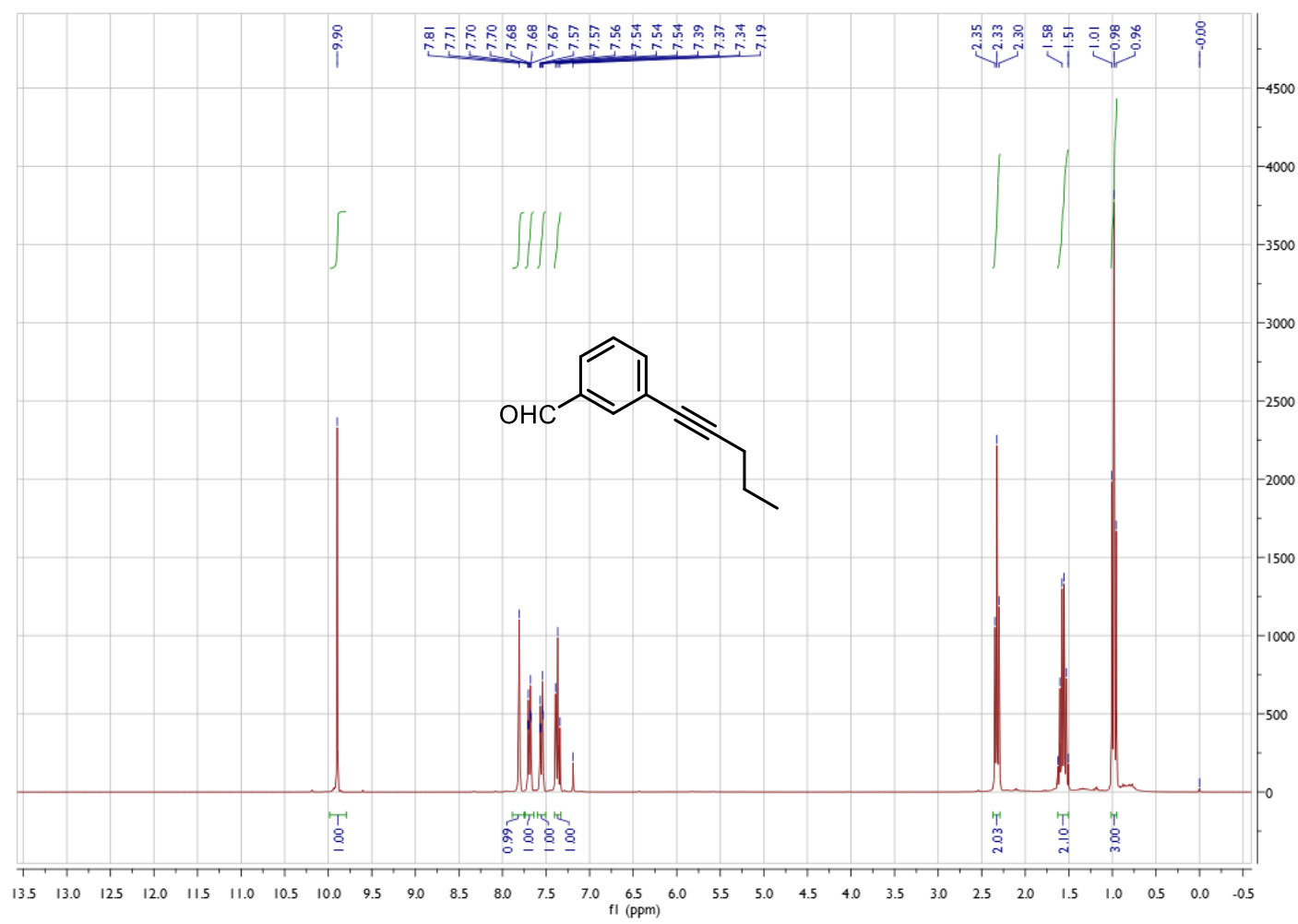

${ }^{13}$ C NMR Spectrum of 3-(Pent-1-yn-1-yl)benzaldehyde 3m

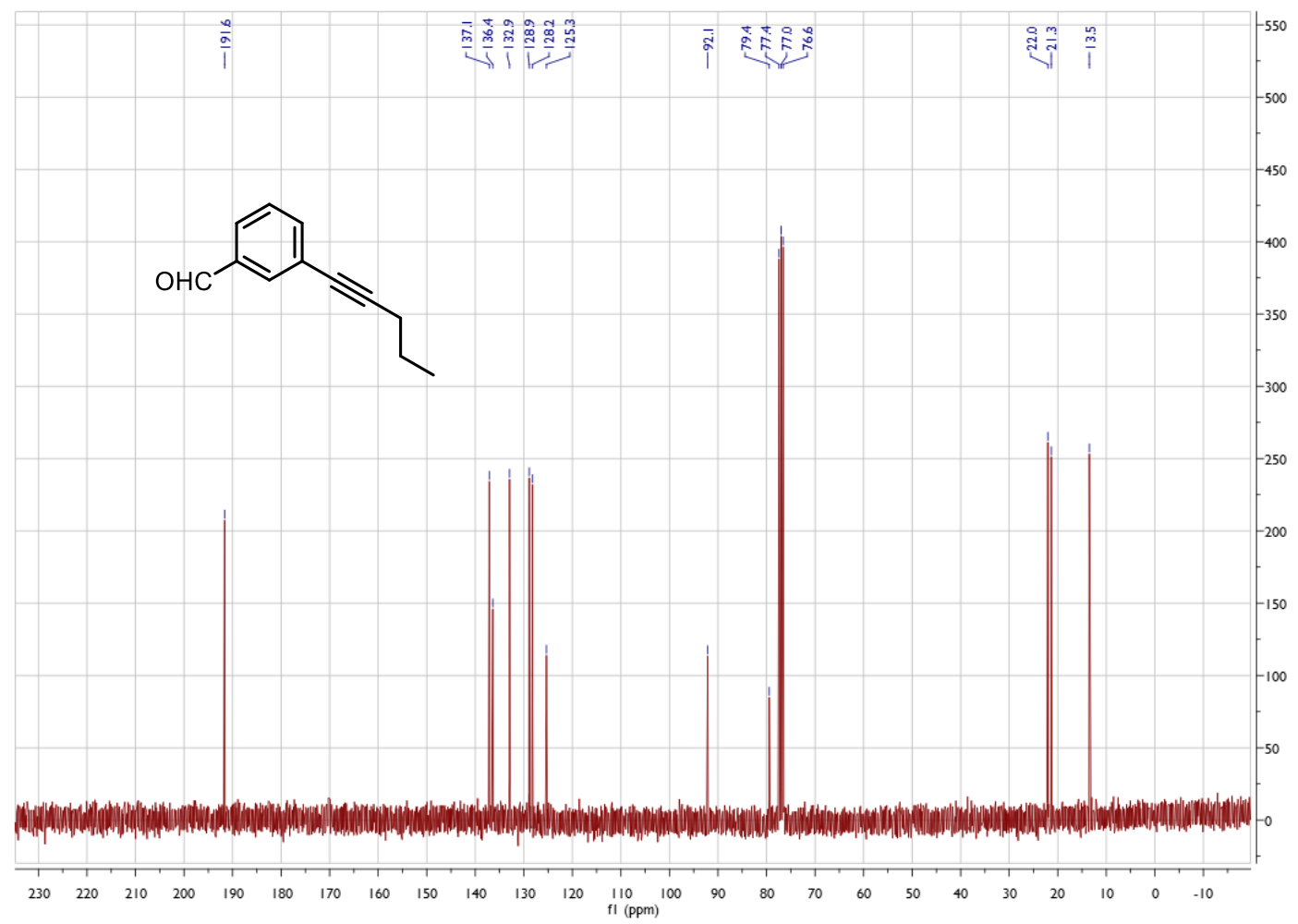


${ }^{1}$ H NMR Spectrum of 1-methyl-2-(pent-1-yn-1-yl)benzene 3n

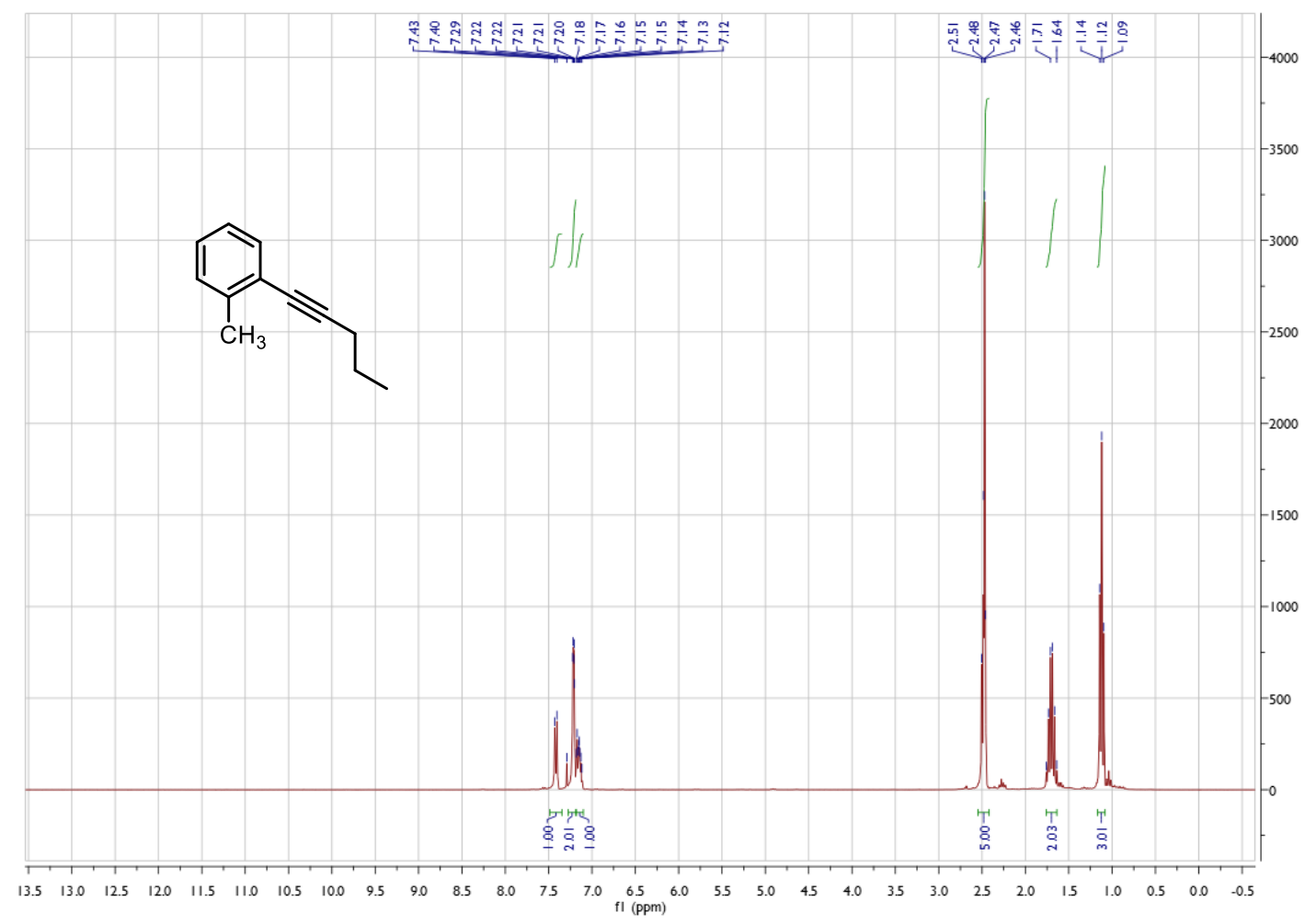

${ }^{13}$ C NMR Spectrum of1-methyl-2-(pent-1-yn-1-yl)benzene 3n

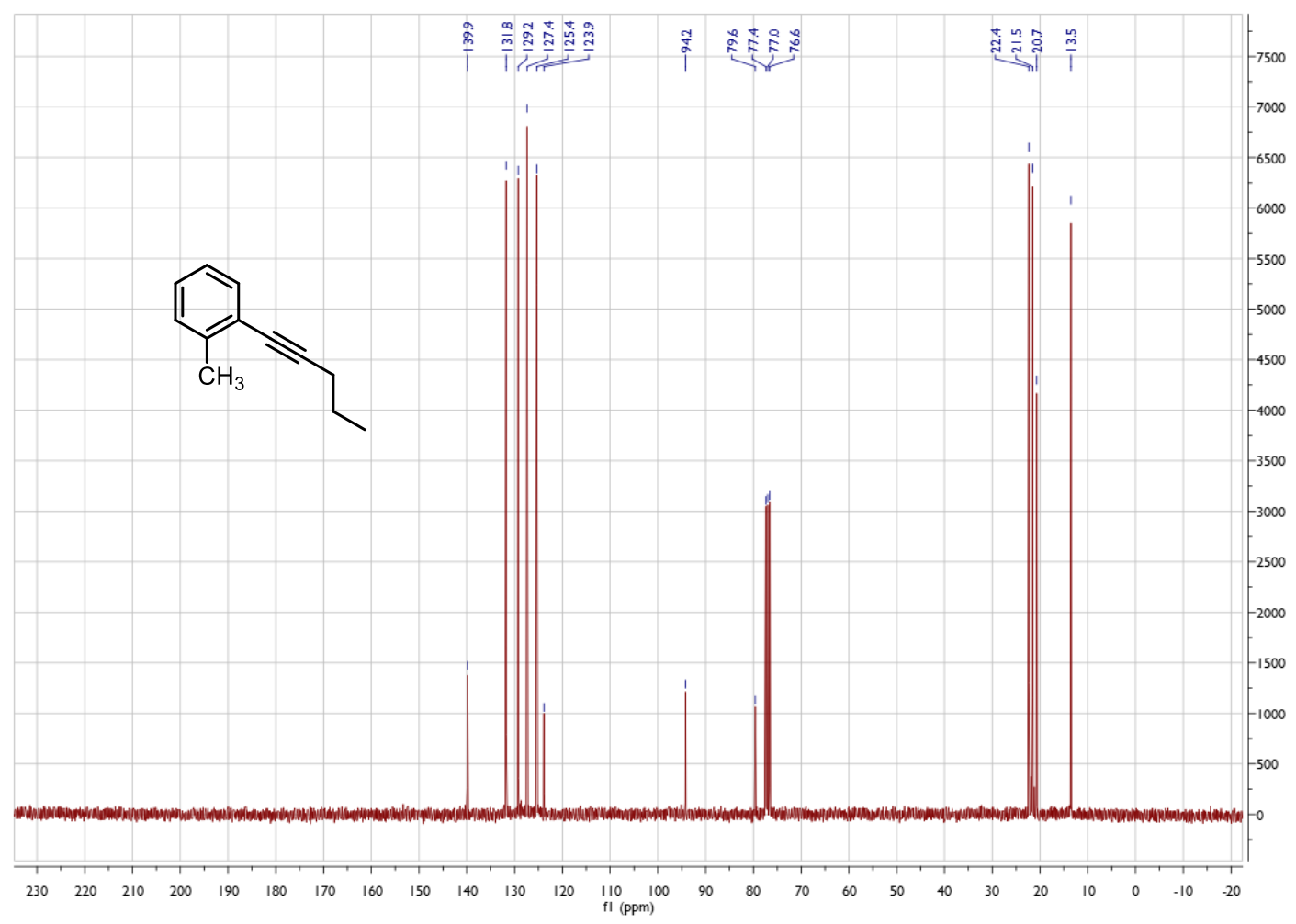




\section{${ }^{1}$ H NMR Spectrum of 1-bromo-4-(hepta-1, 3-diyn-1-yl)benzene 30}

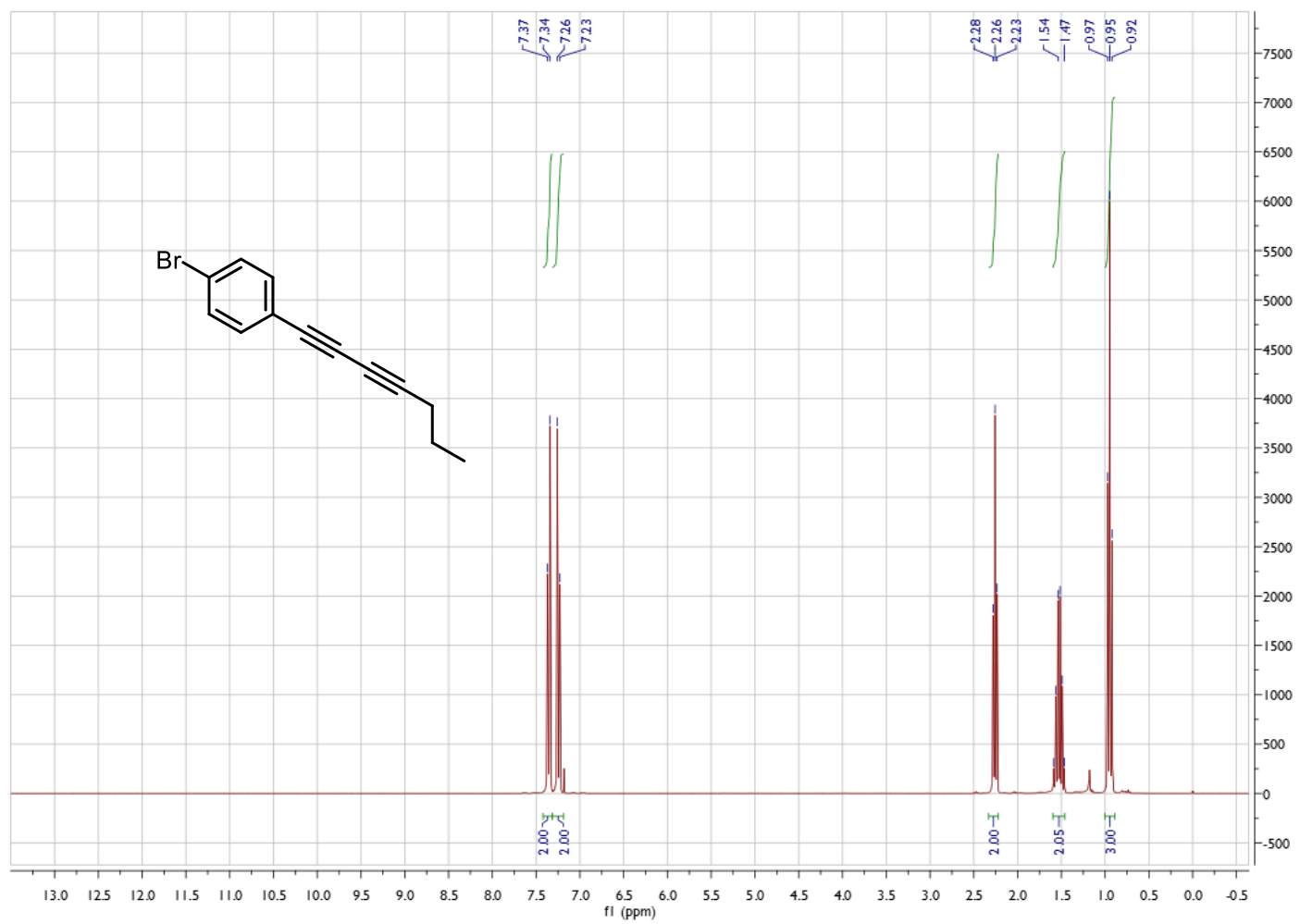

${ }^{13}$ C NMR Spectrum of 1-bromo-4-(hepta-1, 3-diyn-1-yl)benzene 30

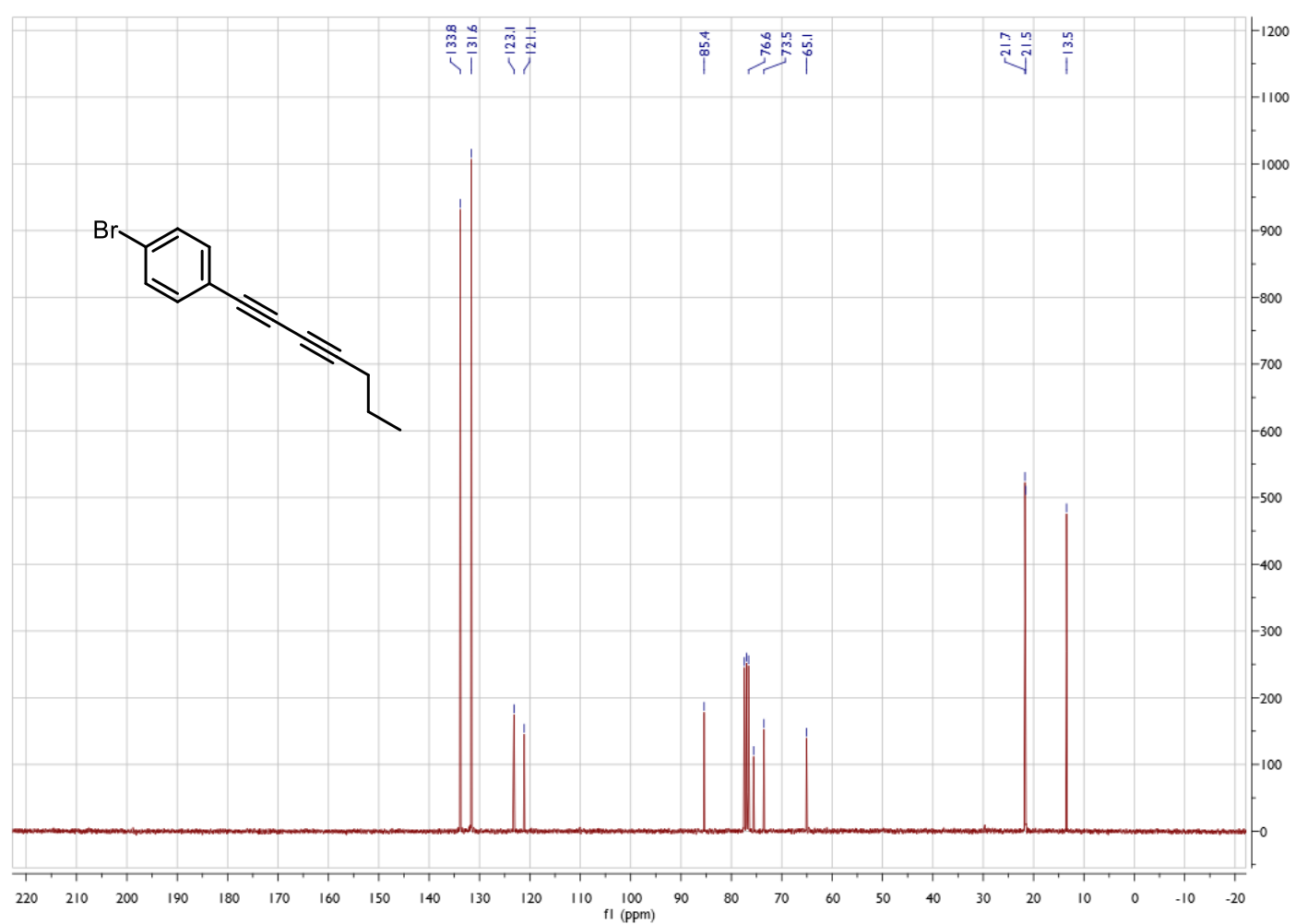


${ }^{1}$ H NMR Spectrum of (E)-1-chloro-4-(hept-1-en-3-yn-1-yl)benzene Bp

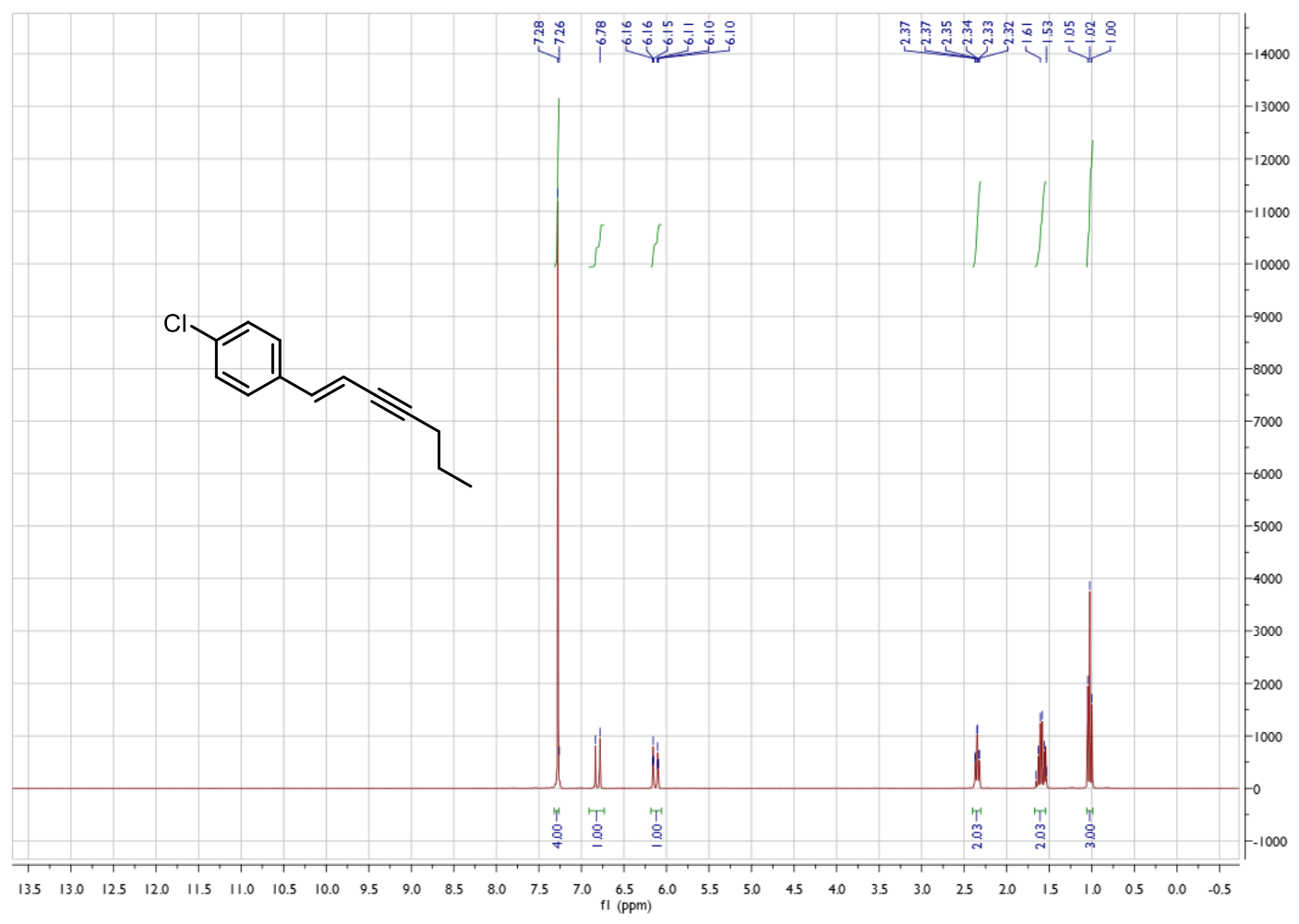

${ }^{13}$ C NMR Spectrum of (E)-1-chloro-4-(hept-1-en-3-yn-1-yl)benzene Bp

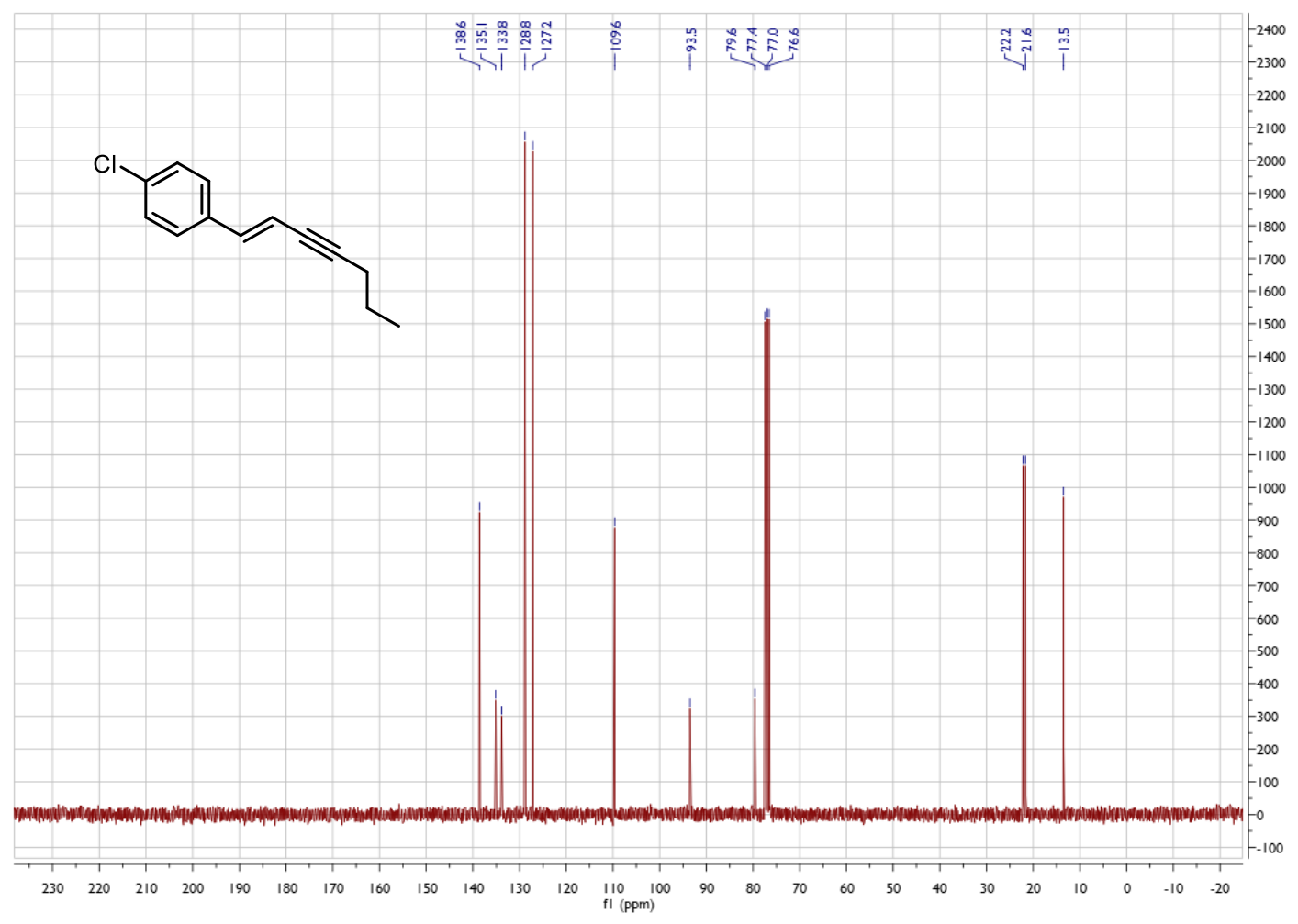


${ }^{1}$ H NMR Spectrum of 1-bromo-4-(5-chloropent-1-yn-1-yl)benzene 3q

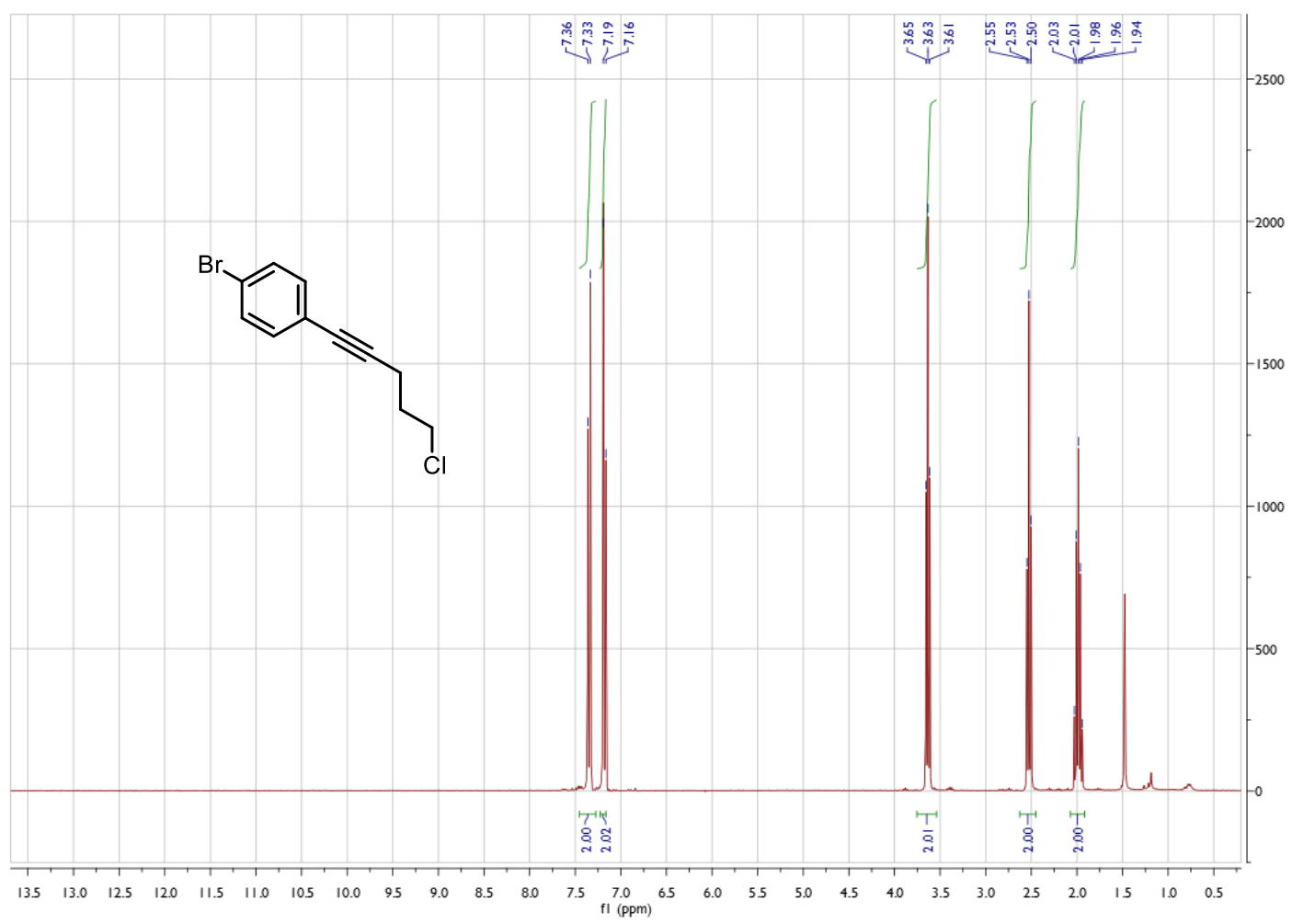

${ }^{13}$ C NMR Spectrum of 1-bromo-4-(5-chloropent-1-yn-1-yl)benzene 3q

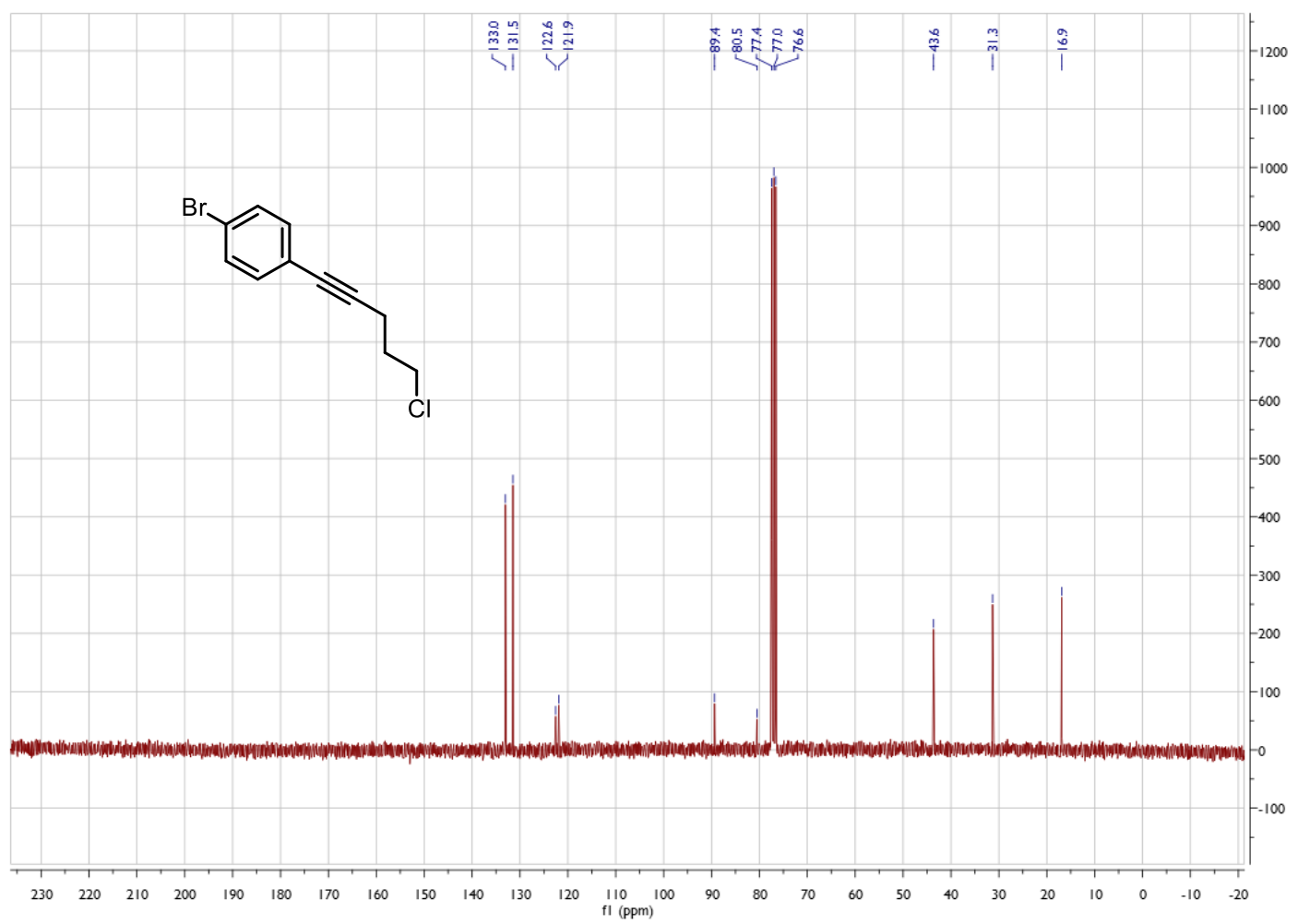


${ }^{1}$ H NMR Spectrum of 4-(4-bromophenyl)but-3-yn-1-yl 4-methylbenzenesulfonate 3r

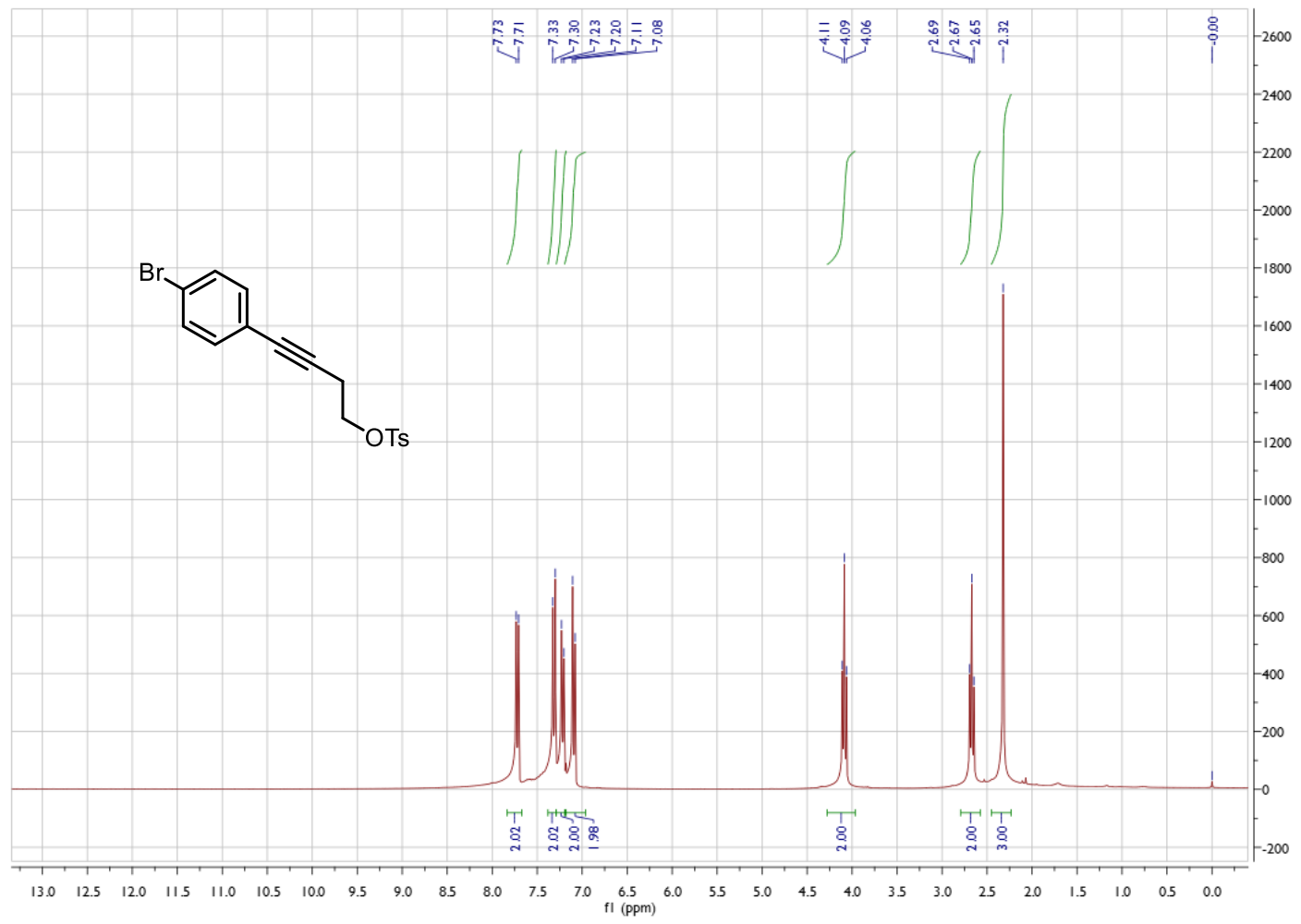

${ }^{13}$ C NMR Spectrum of 4-(4-bromophenyl)but-3-yn-1-yl 4-methylbenzenesulfonate $3 r$

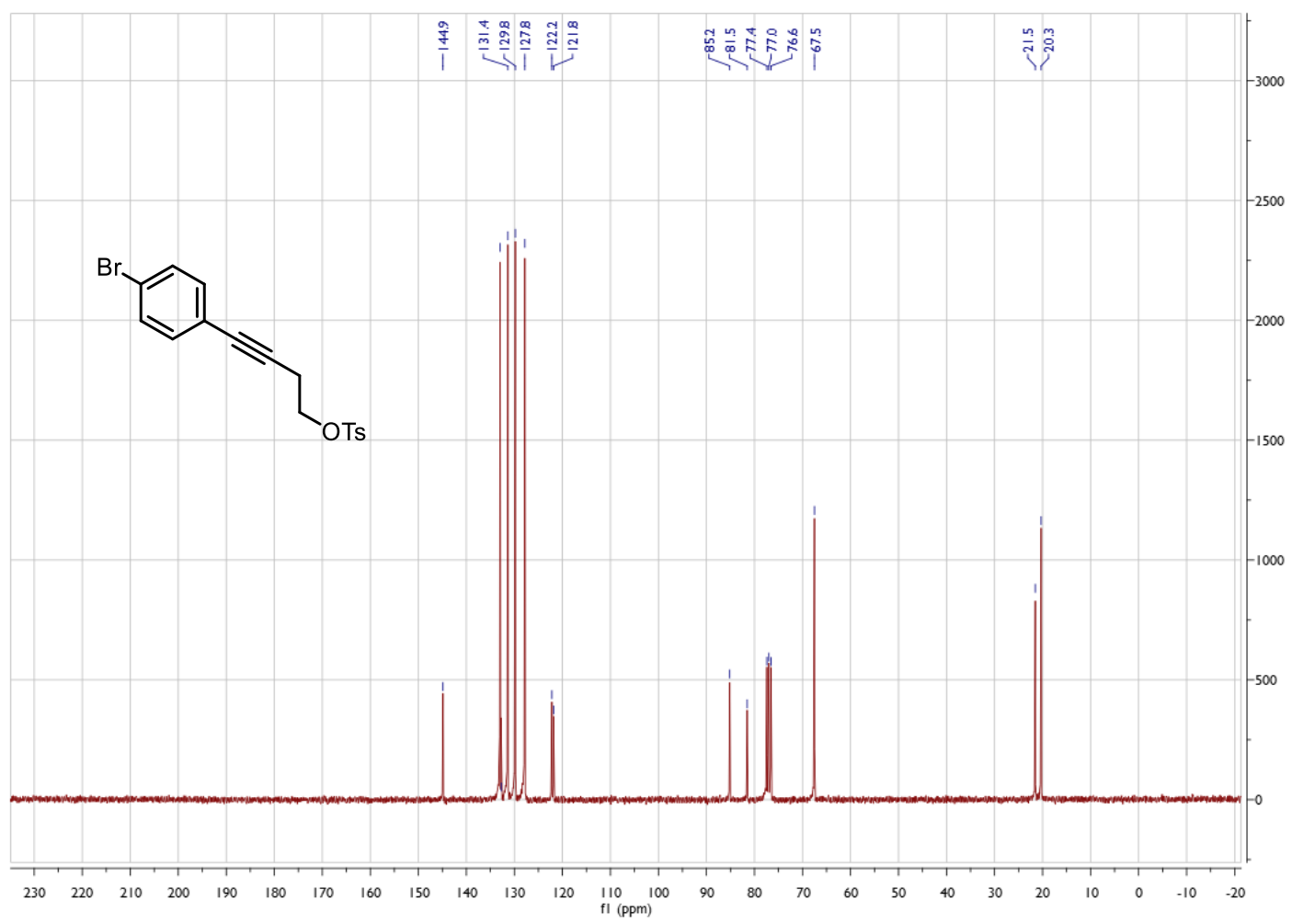


${ }^{1}$ H NMR Spectrum of 2-(6-(4-bromophenyl)hex-5-yn-1-yl)isoindoline-1,3-dione 3s

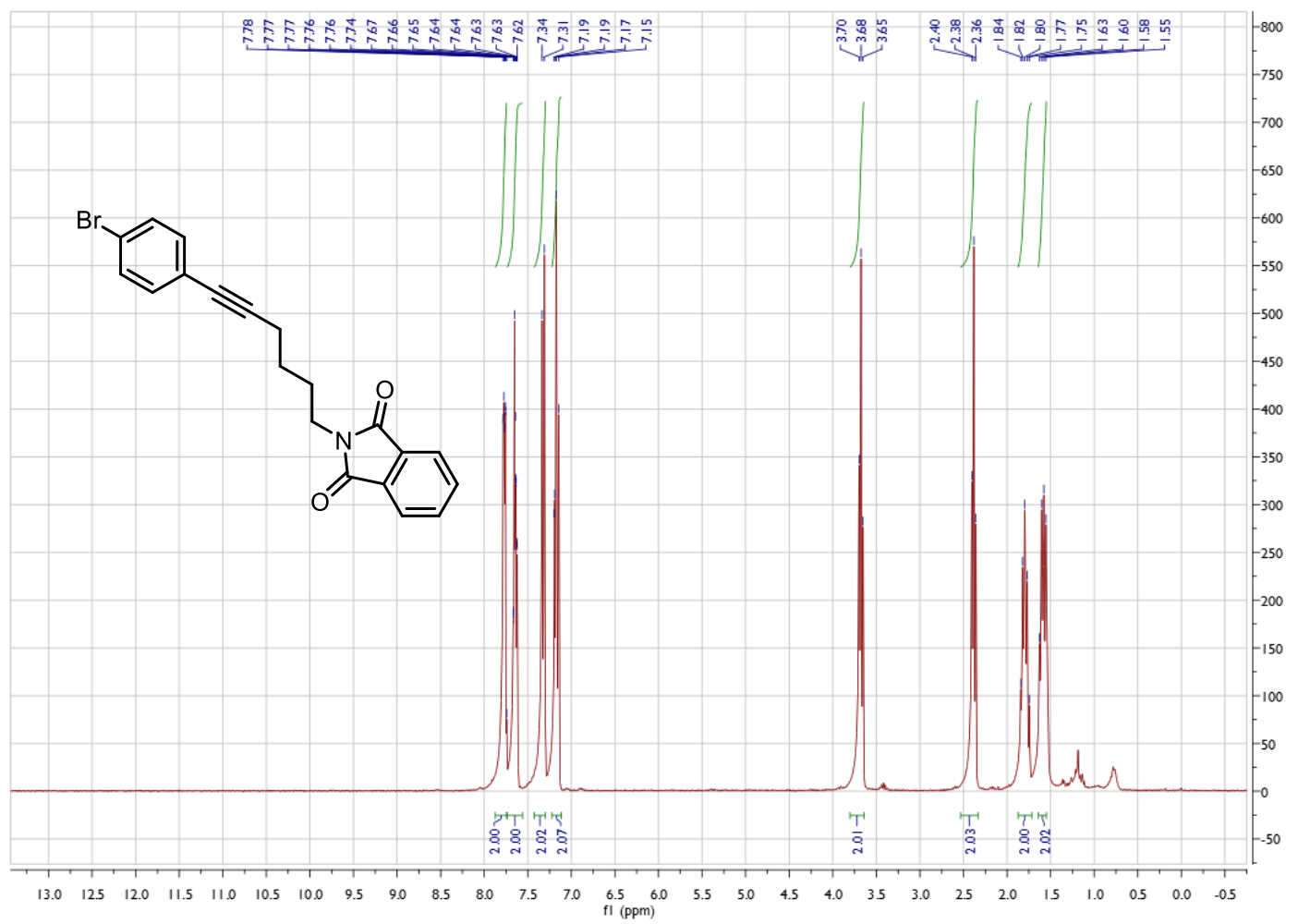

${ }^{13}$ C NMR Spectrum of 2-(6-(4-bromophenyl)hex-5-yn-1-yl)isoindoline-1,3-dione 3s

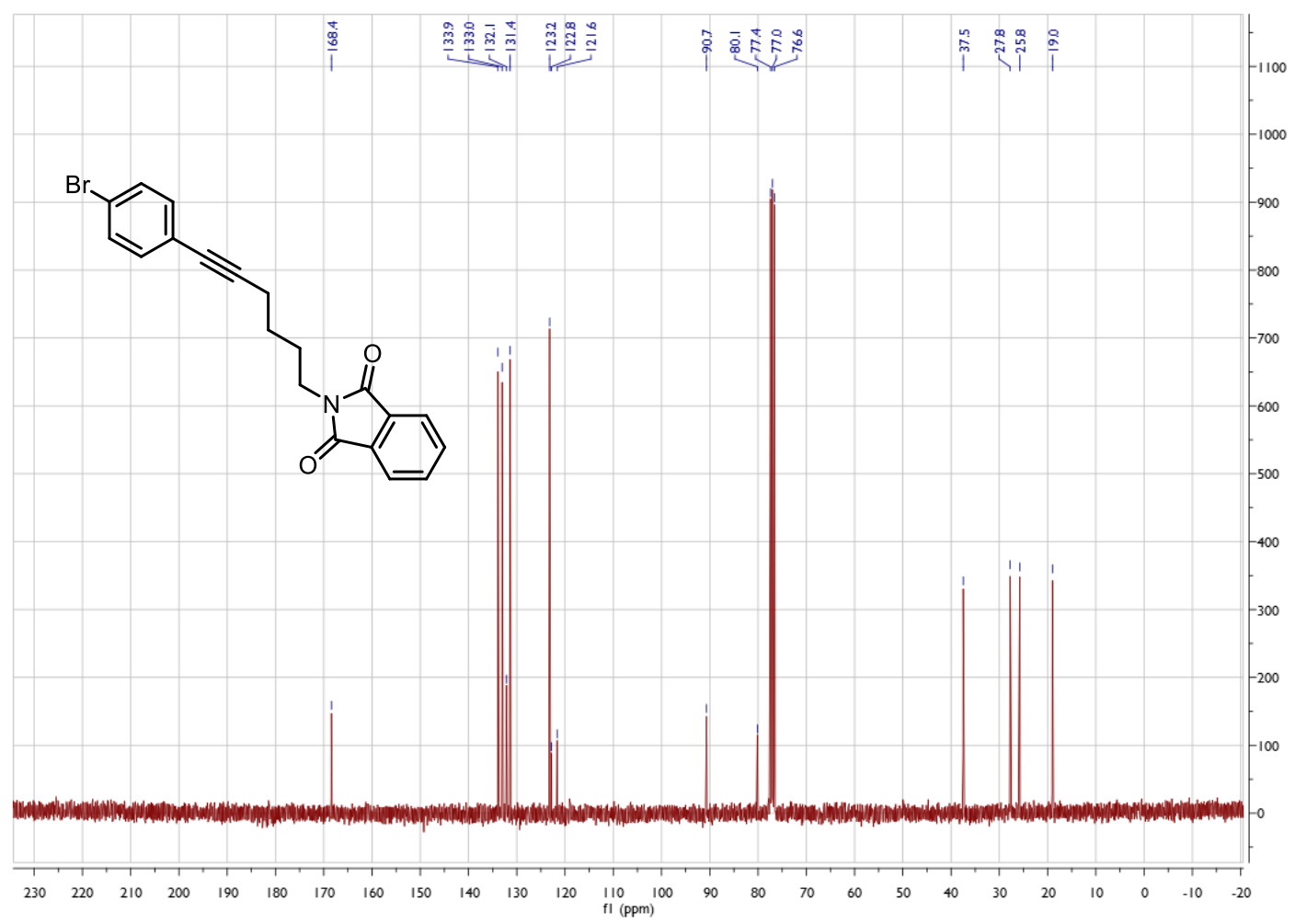


${ }^{1}$ H NMR Spectrum of 1-bromo-4-(prop-1-yn-1-yl)benzene Bt

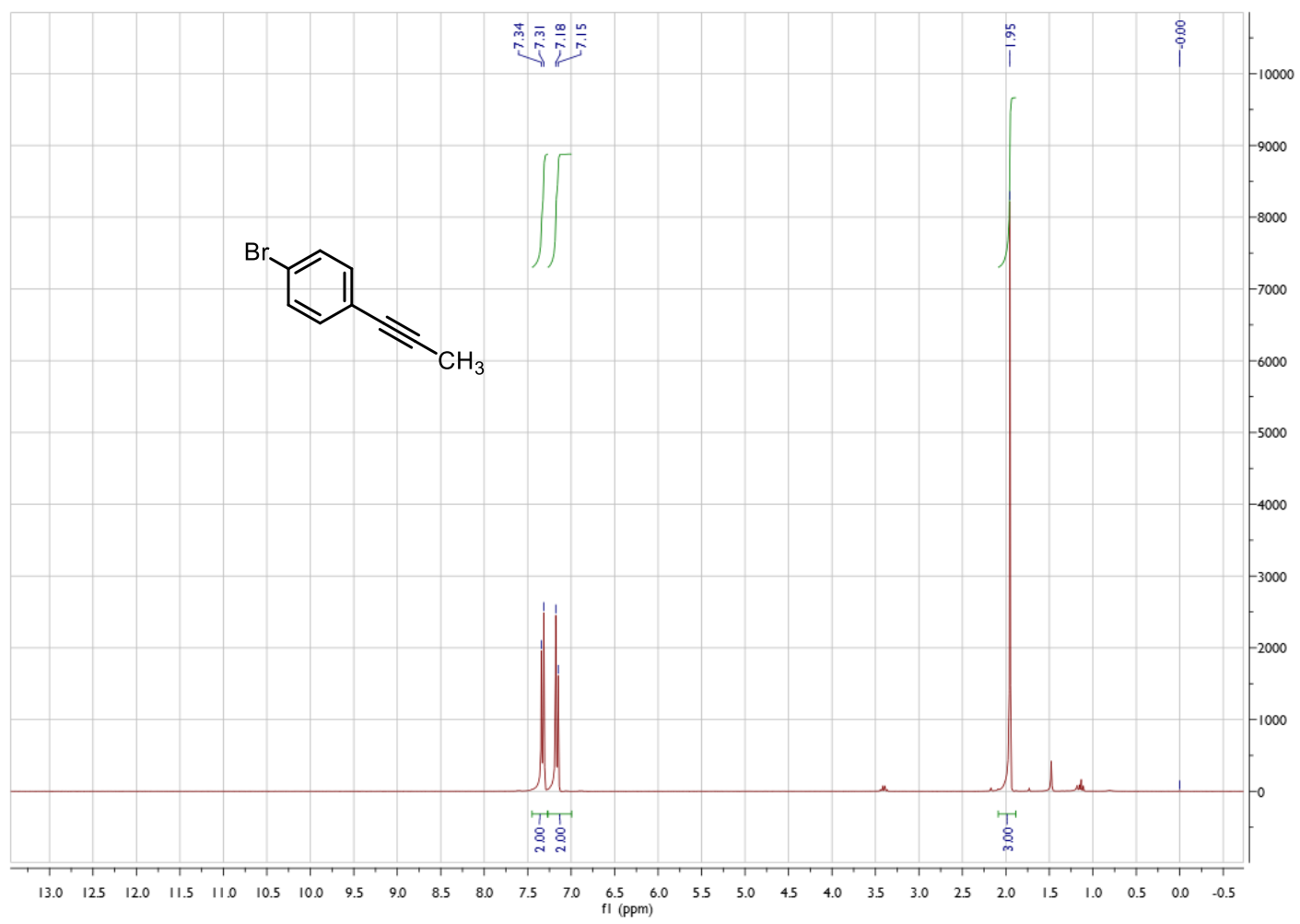

${ }^{13}$ C NMR Spectrum of 1-bromo-4-(prop-1-yn-1-yl)benzene Bt

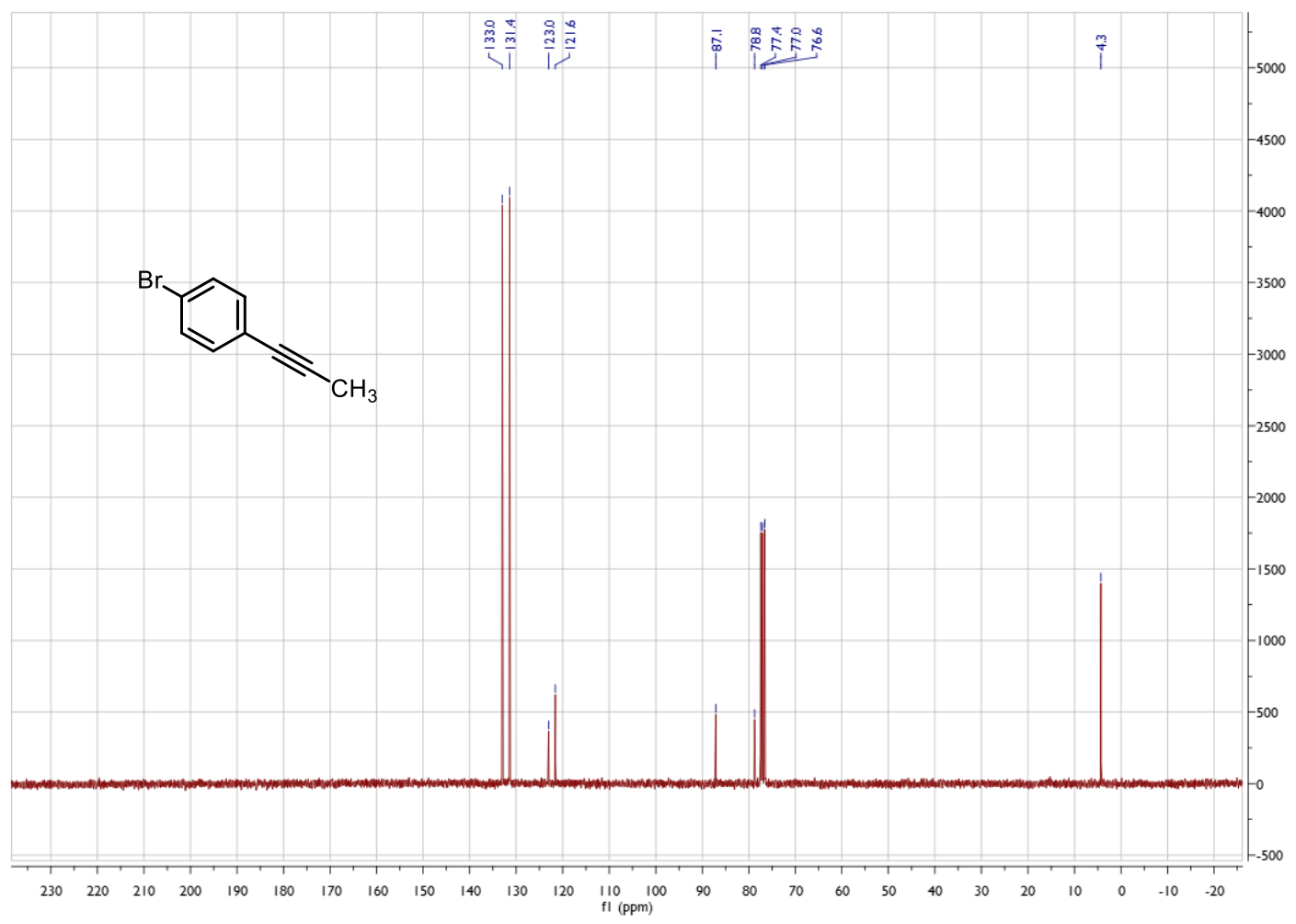


${ }^{1}$ H NMR Spectrum of 1-bromo-4-(3-methylbut-1-yn-1-yl)benzene 3u

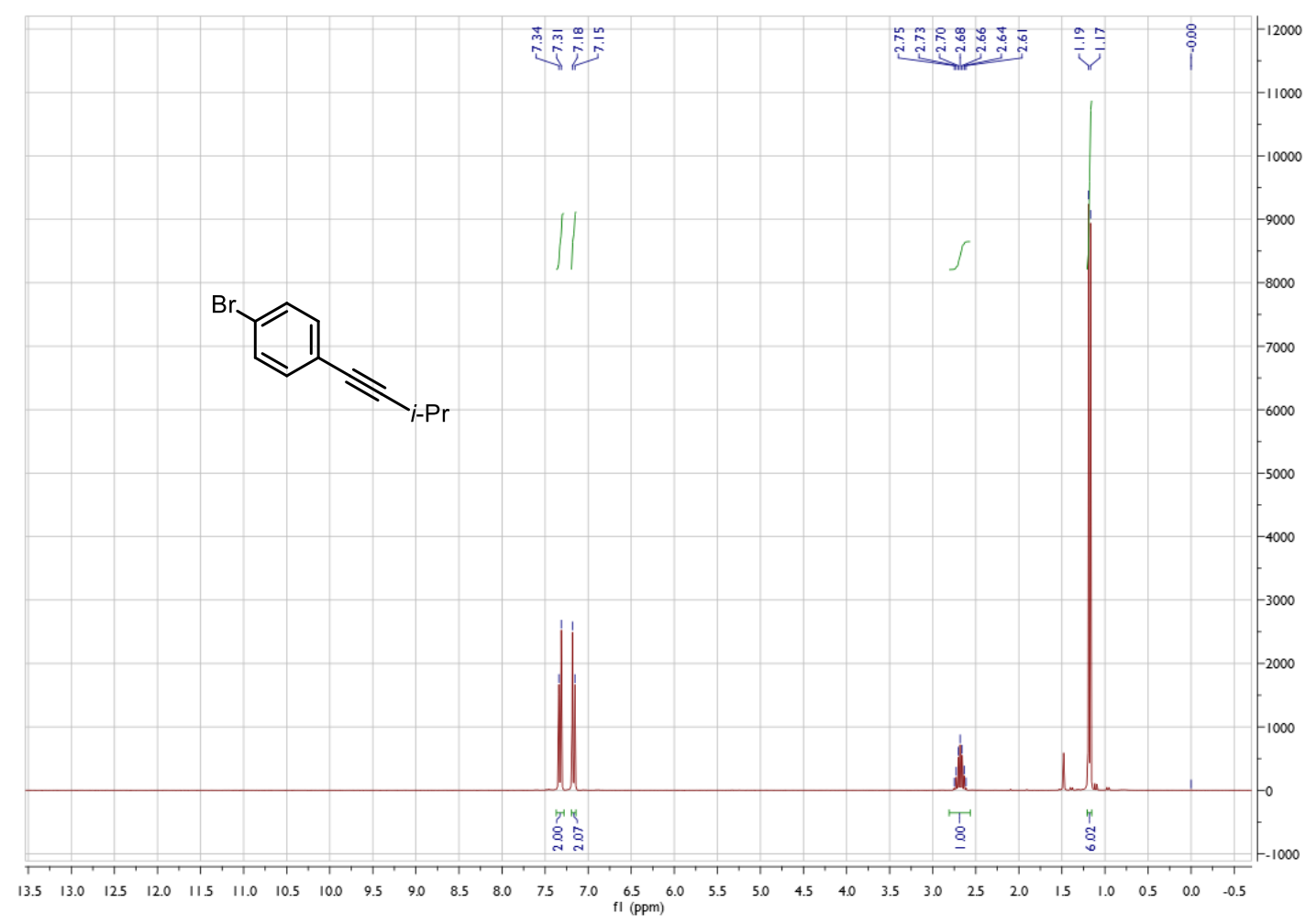

${ }^{13}$ C NMR Spectrum of 1-bromo-4-(3-methylbut-1-yn-1-yl)benzene 3u

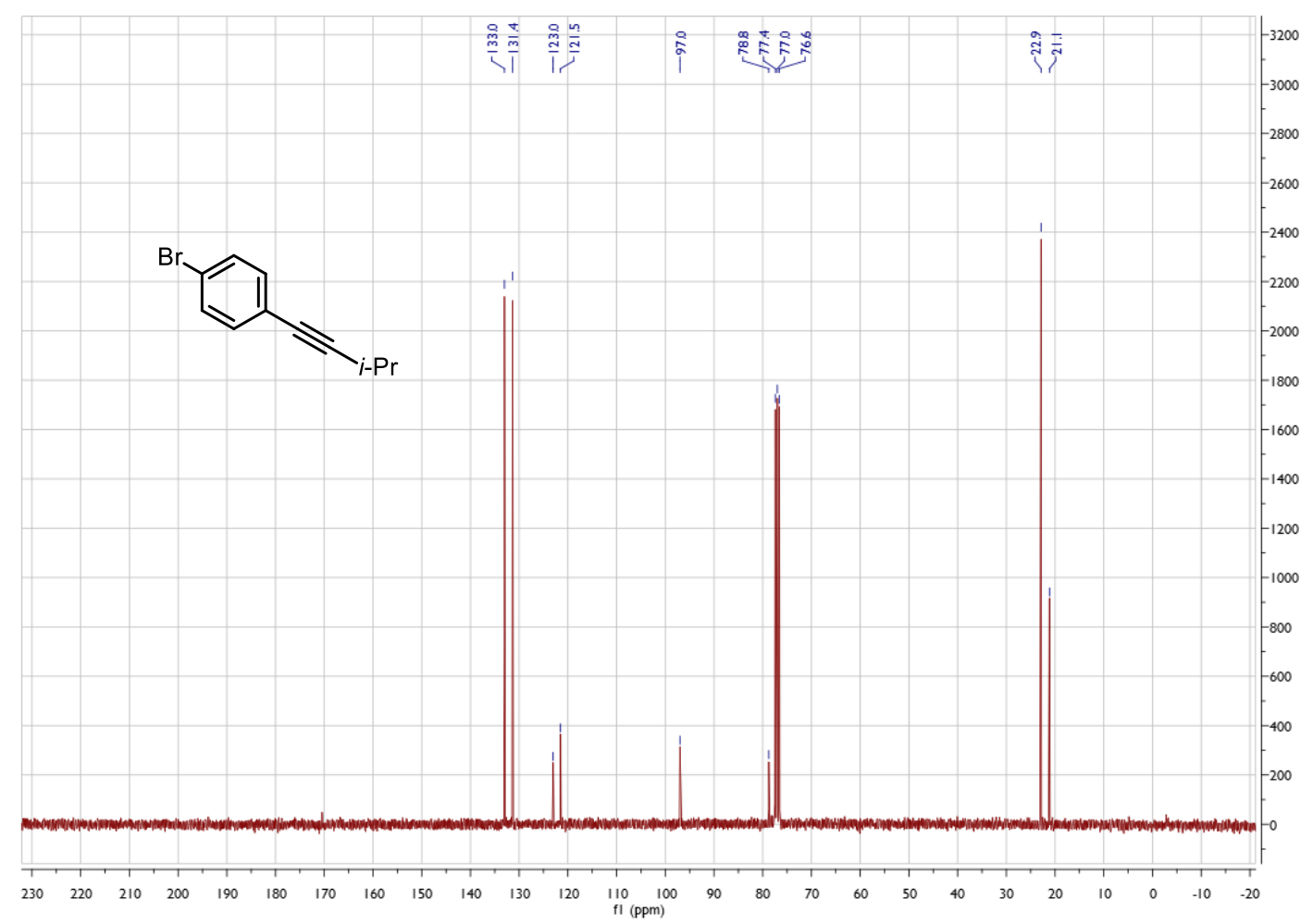


${ }^{1} \mathrm{H}$ NMR Spectrum of 1-bromo-4-(3, 3-dimethylbut-1-yn-1-yl)benzene 3v

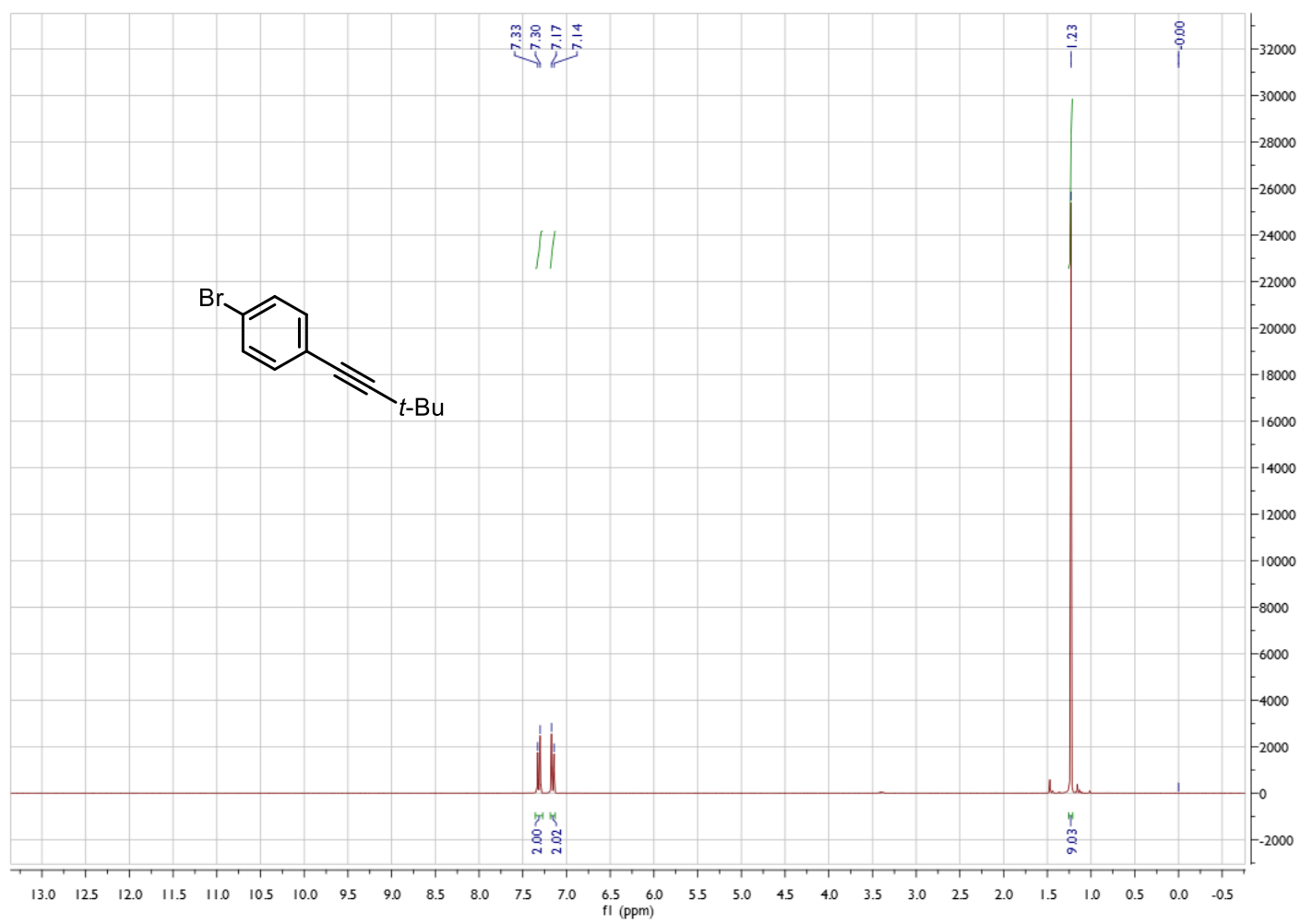

${ }^{13}$ C NMR Spectrum of 1-bromo-4-(3, 3-dimethylbut-1-yn-1-yl)benzene 3v

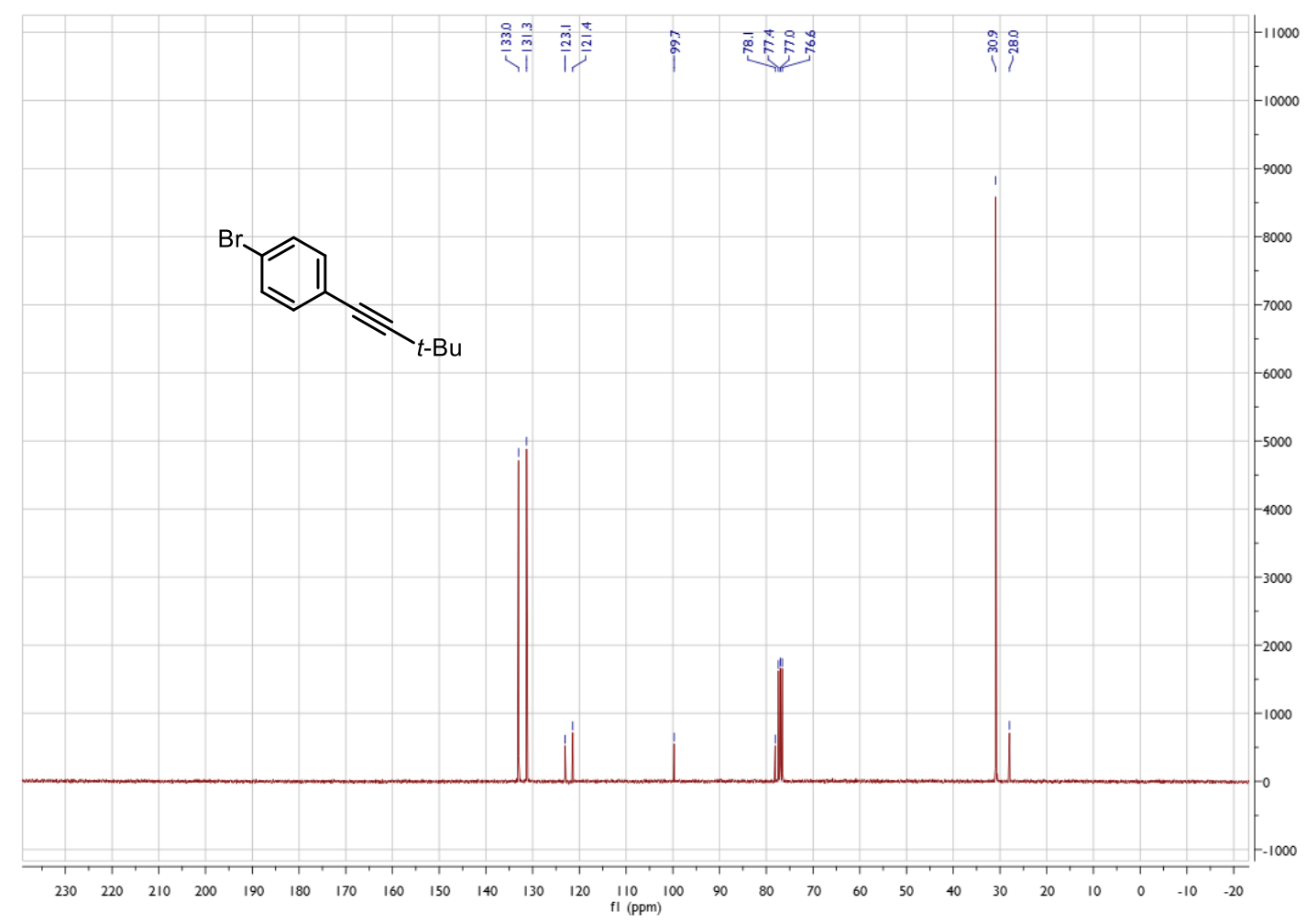


${ }^{1}$ H NMR Spectrum of 1-bromo-4-(cyclopropylethynyl)benzene 3w

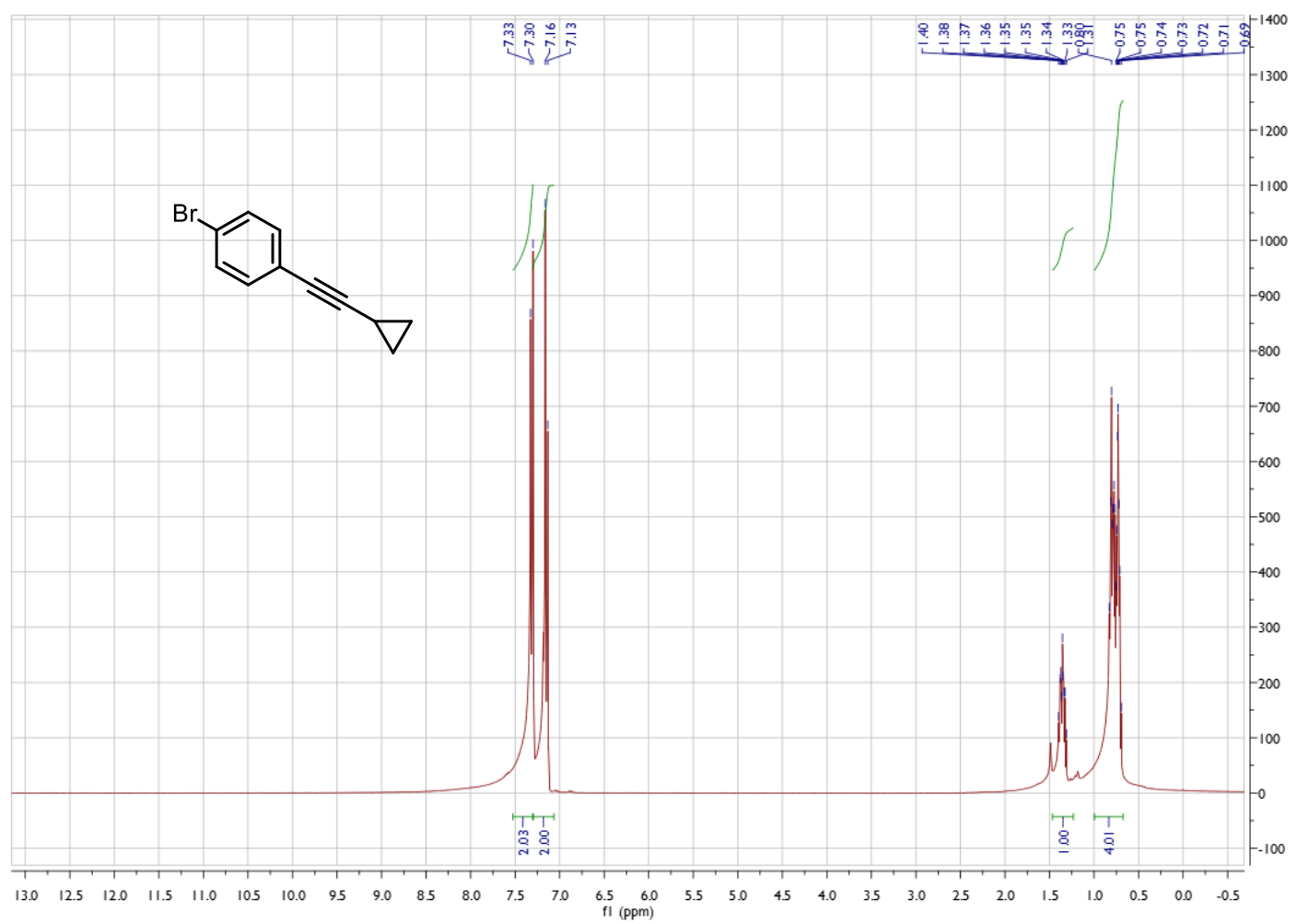

${ }^{13}$ C NMR Spectrum of 1-bromo-4-(cyclopropylethynyl)benzene 3w

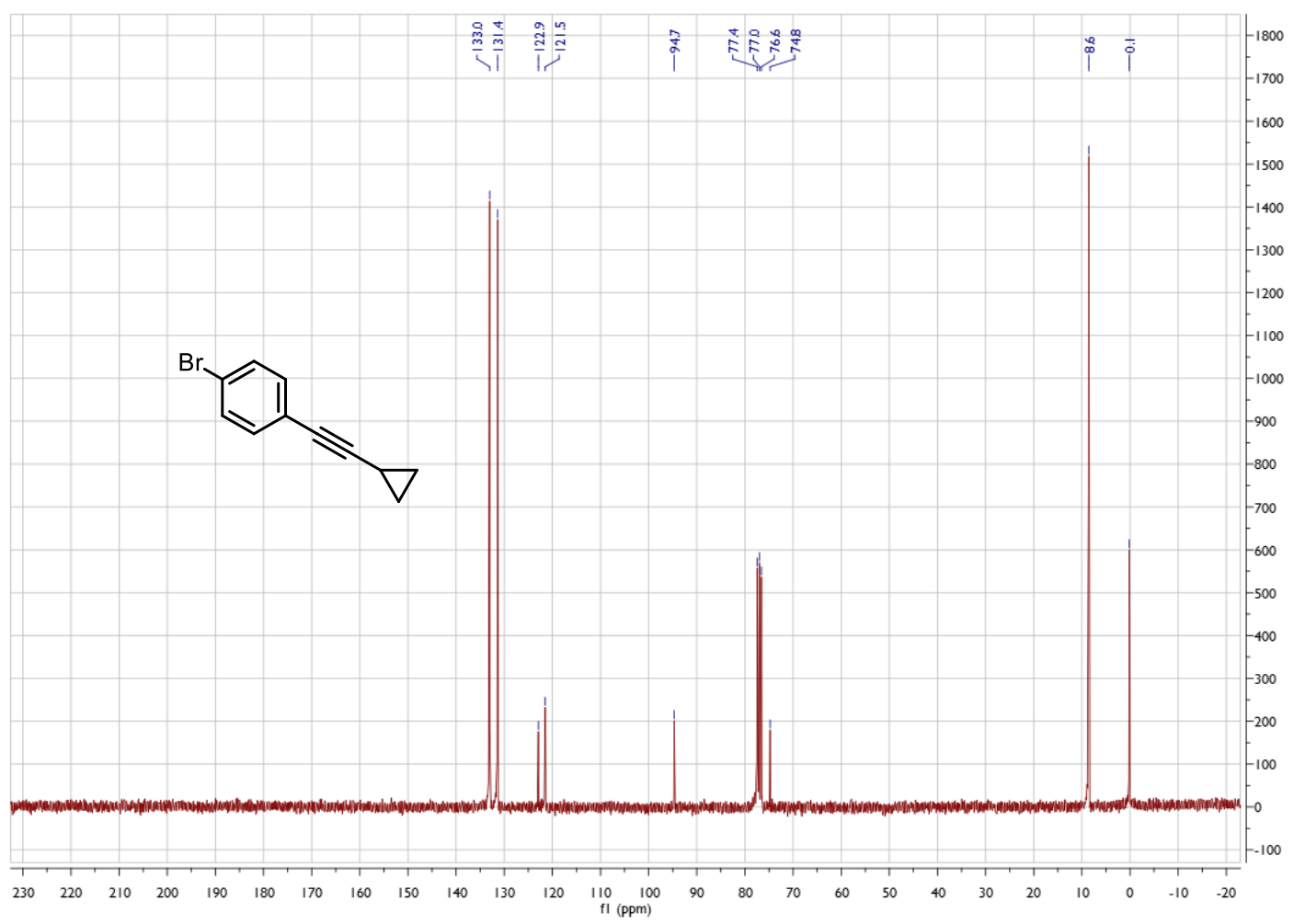


${ }^{1}$ H NMR Spectrum of 1-bromo-4-(cyclopentylethynyl)benzene 3x

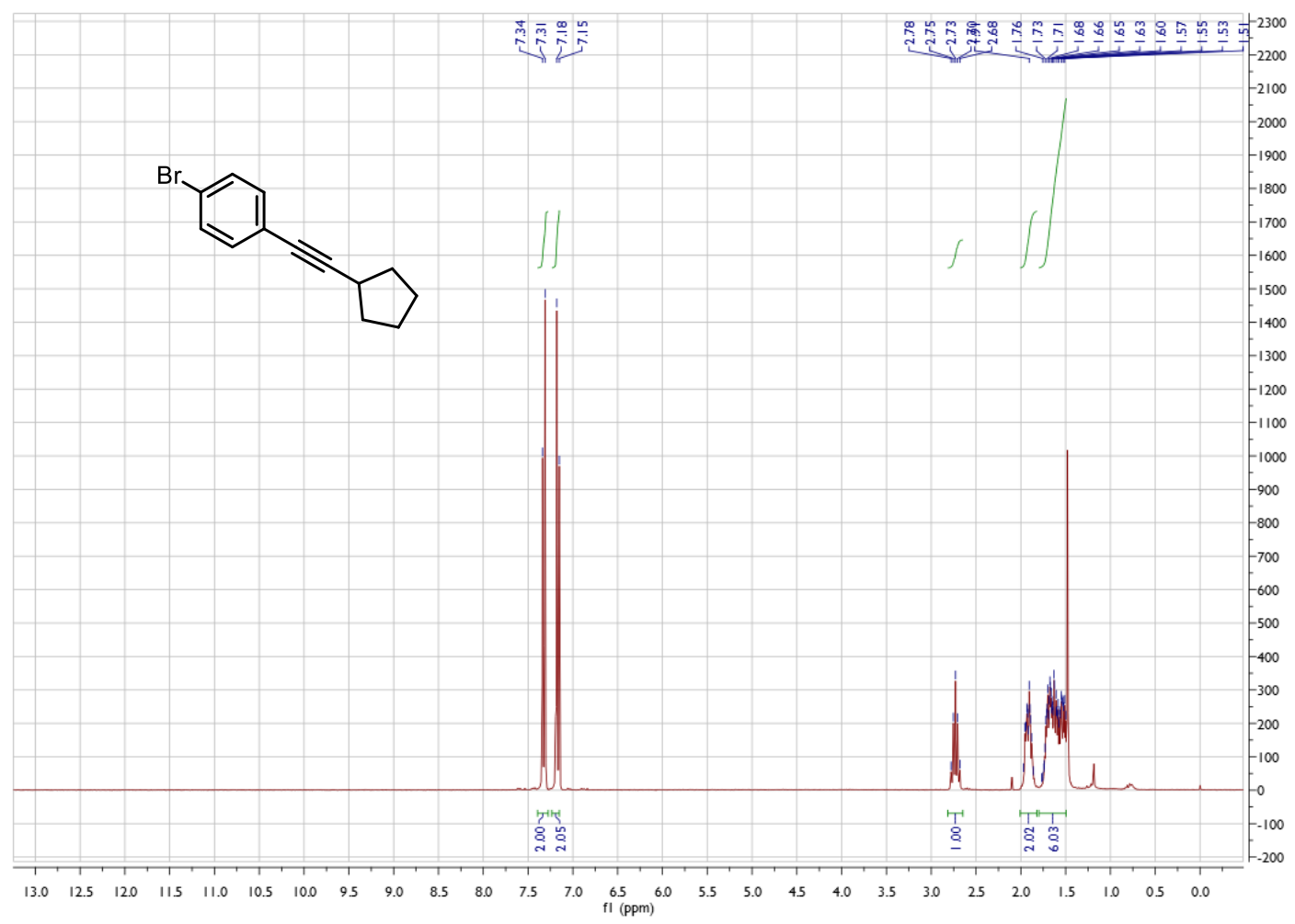

${ }^{13}$ C NMR Spectrum of 1-bromo-4-(cyclopentylethynyl)benzene $3 x$

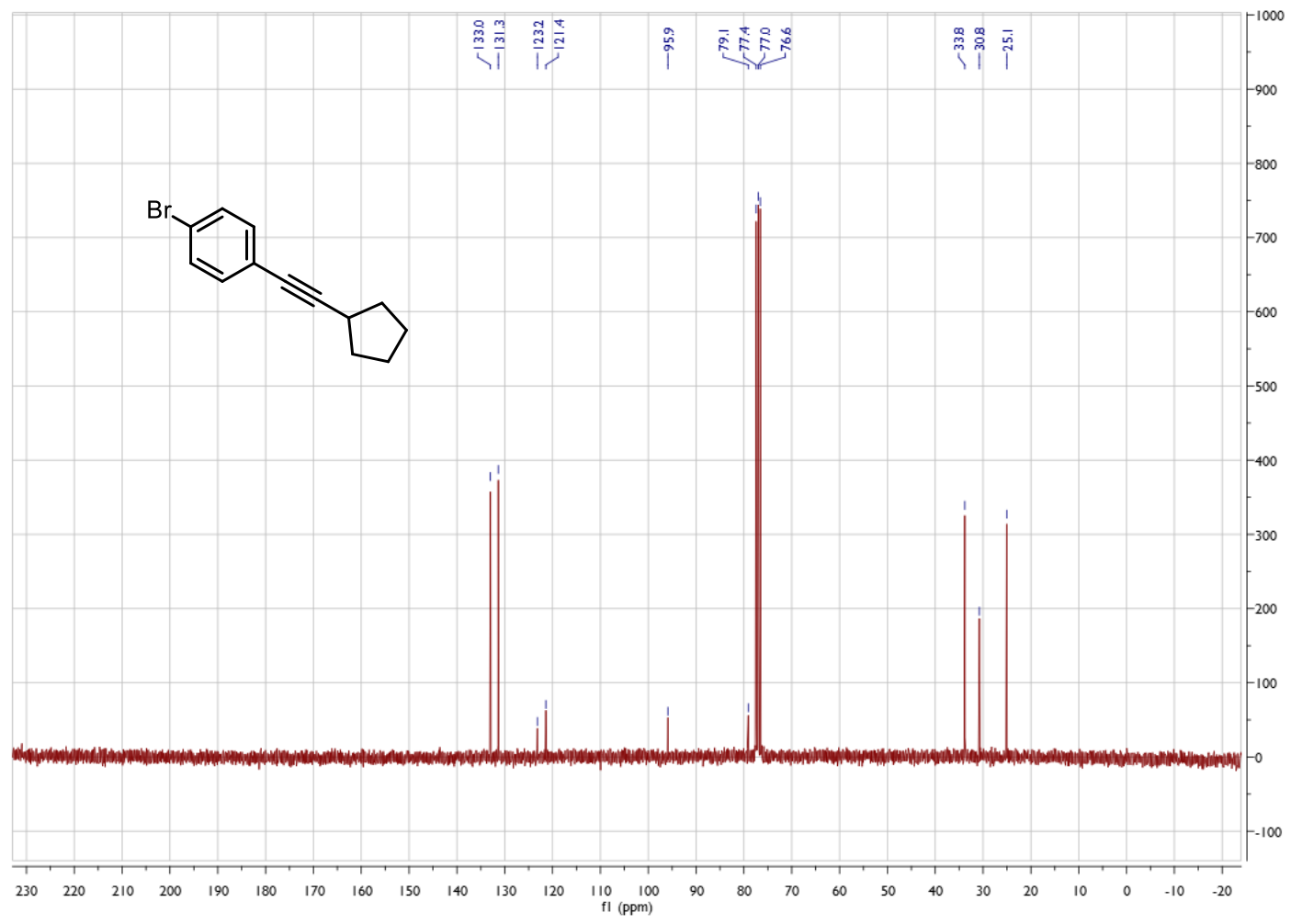


${ }^{1}$ H NMR Spectrum of 1-bromo-4-(cyclohexylethynyl)benzene $3 y$

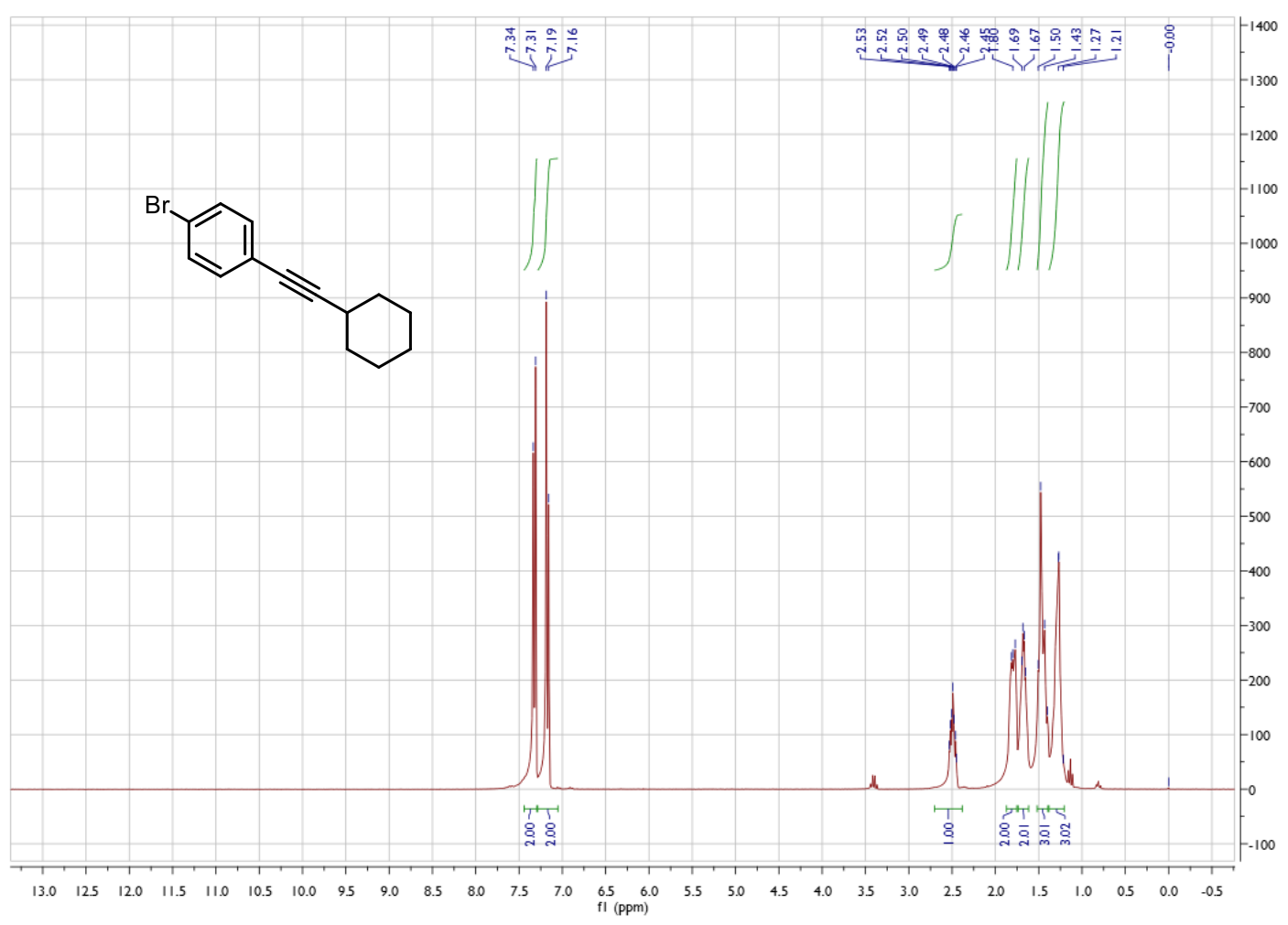

${ }^{13}$ C NMR Spectrum of 1-bromo-4-(cyclohexylethynyl)benzene 3y

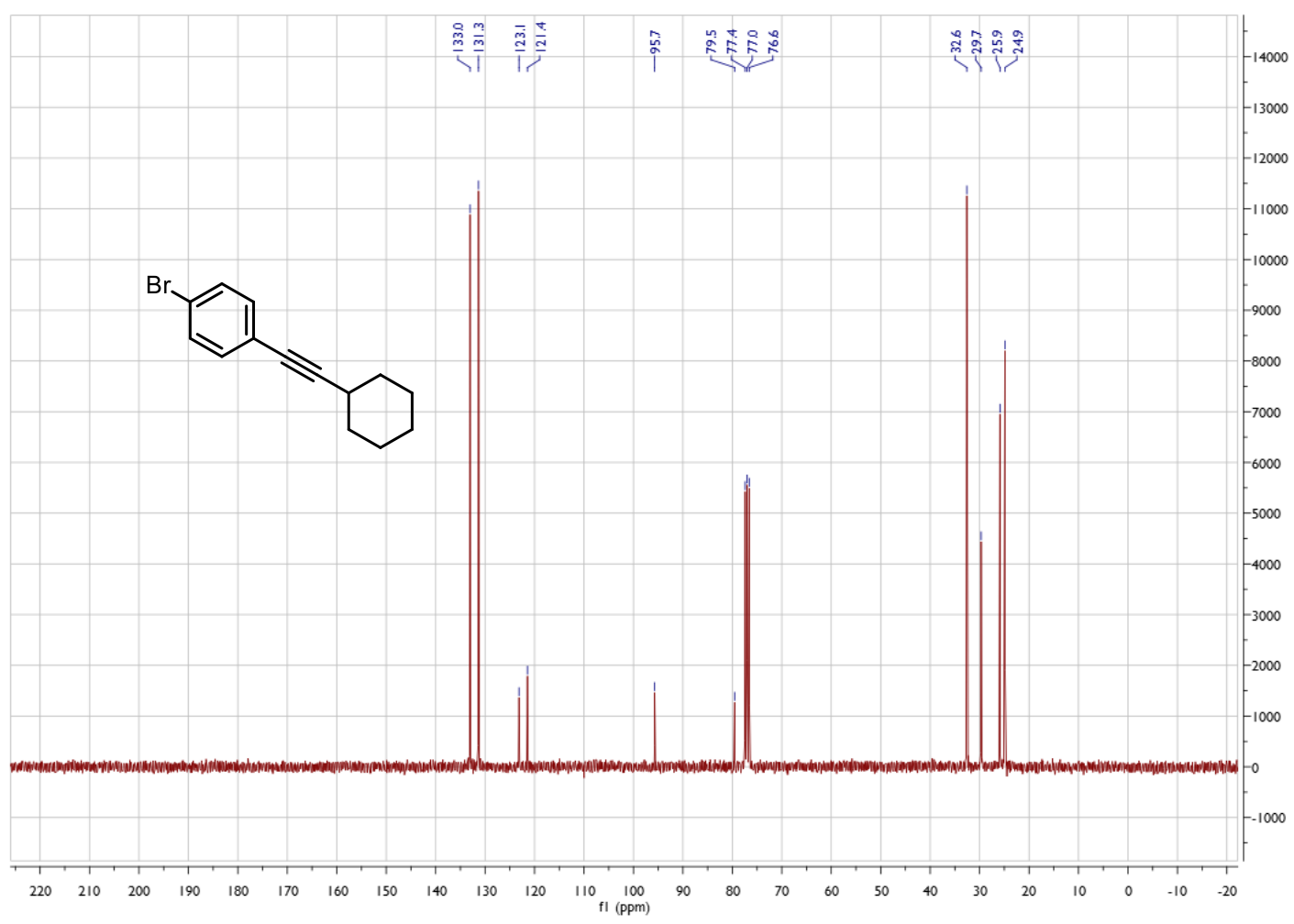


${ }^{1}$ H NMR Spectrum of 1, 6-bis(4-chlorophenyl)hexa-1,5-diyne az

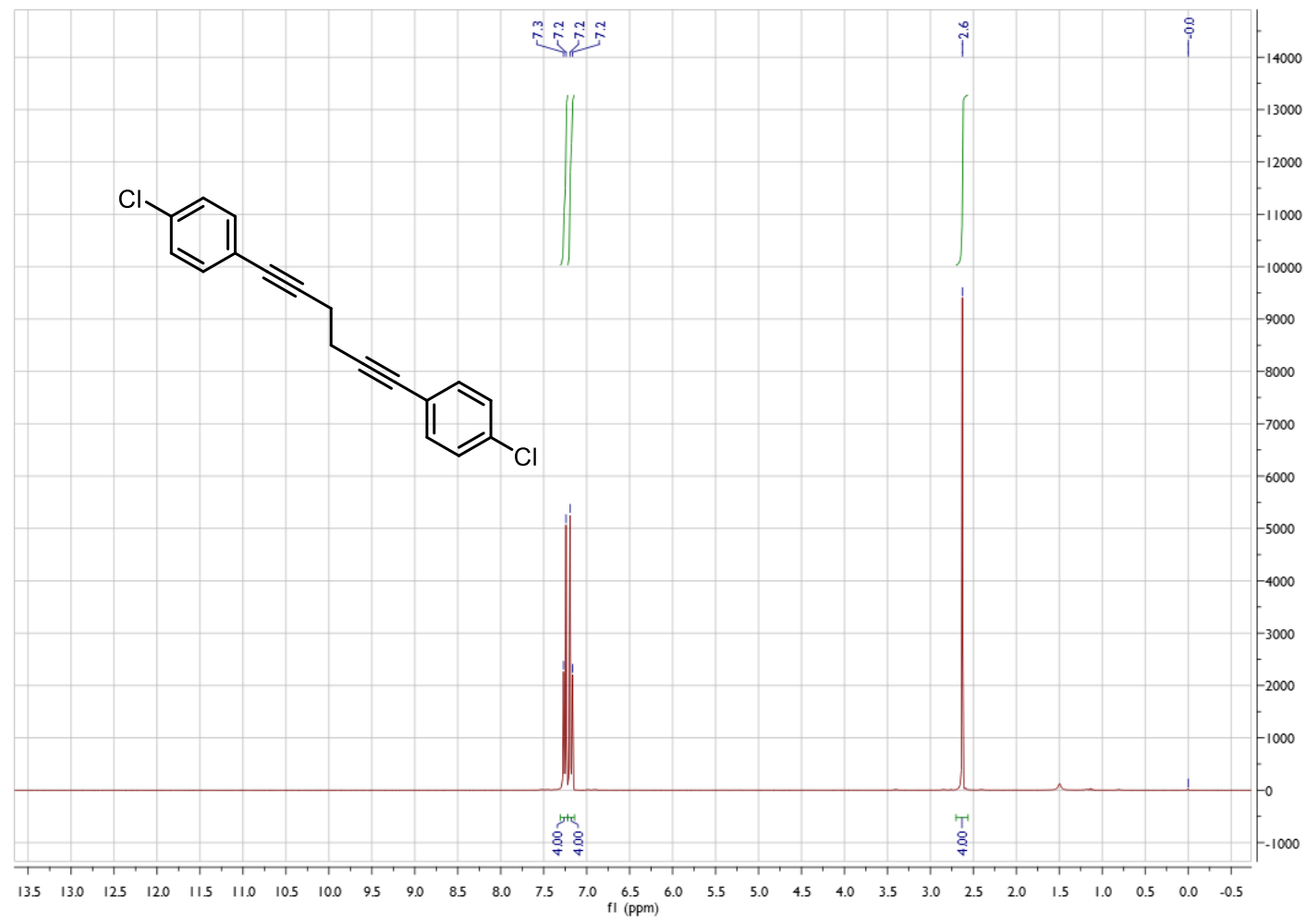

${ }^{13}$ C NMR Spectrum of 1, 6-bis(4-chlorophenyl)hexa-1,5-diyne az

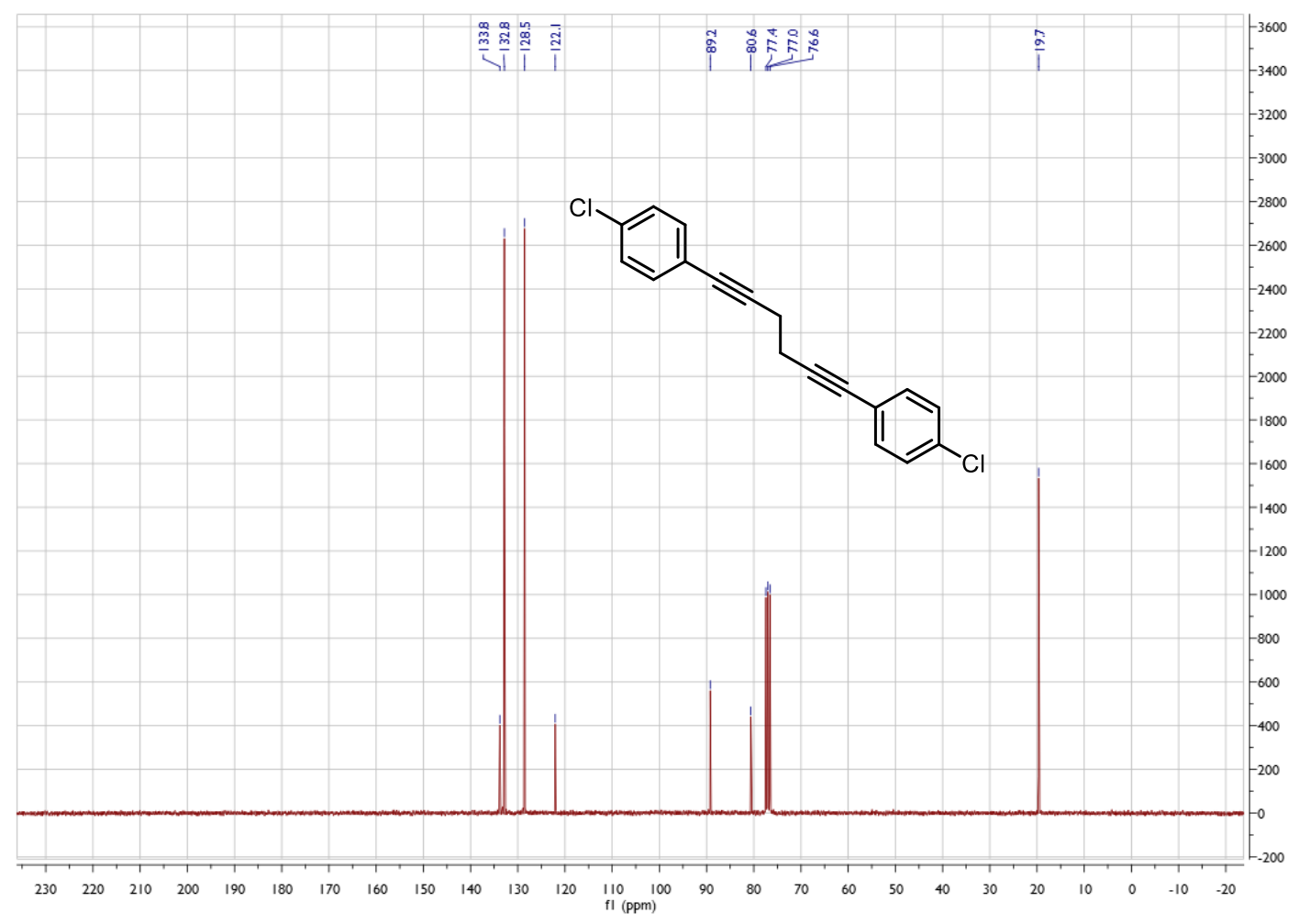


Spectra of cyanotriflation products 2

${ }^{1}$ H NMR Spectrum of (Z)-2-cyano-1-phenylpent-1-en-1-yl trifluoromethanesulfonate $2 \mathrm{a}$

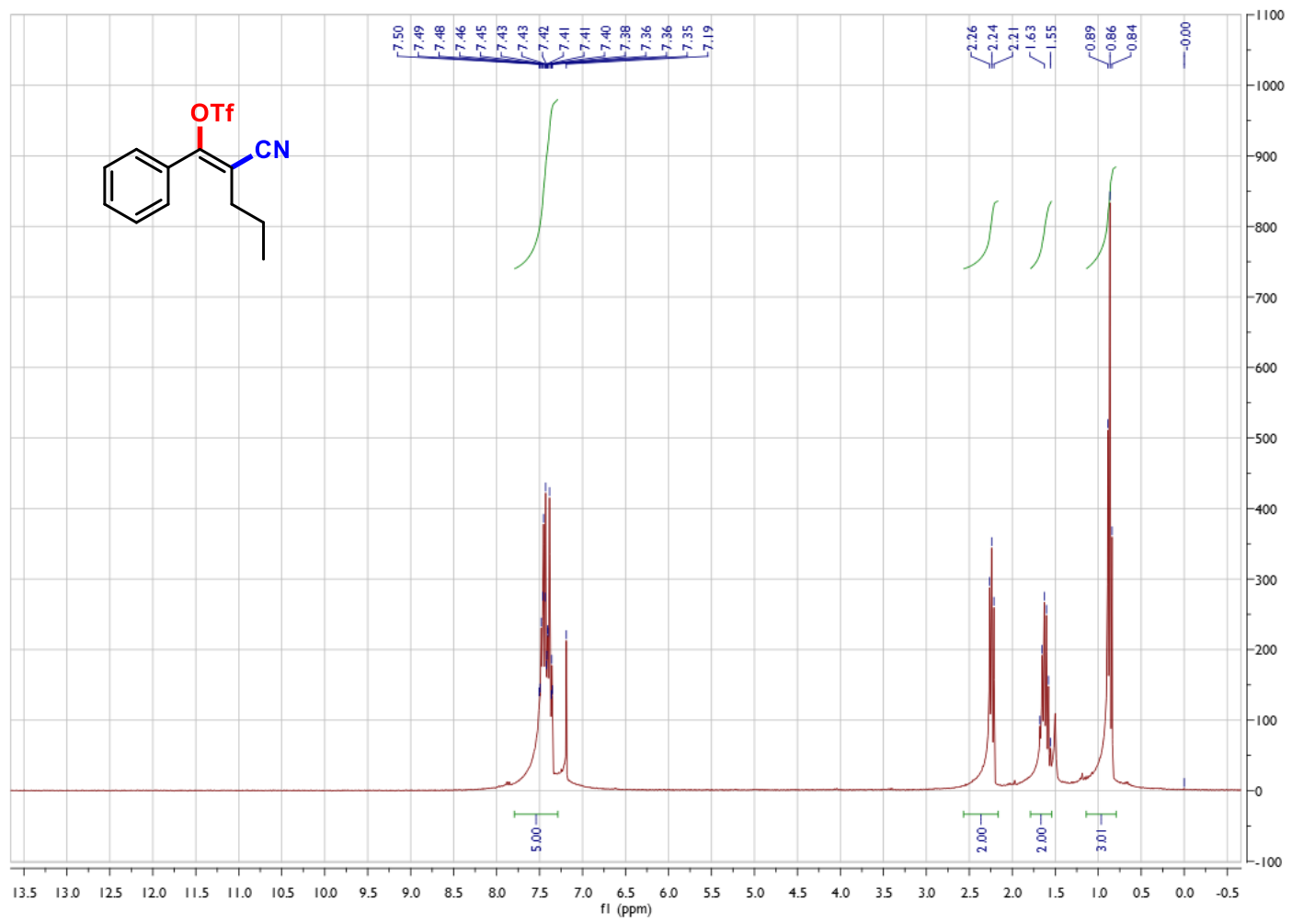

${ }^{13}$ C NMR Spectrum of (Z)-2-cyano-1-phenylpent-1-en-1-yl trifluoromethanesulfonate 2a

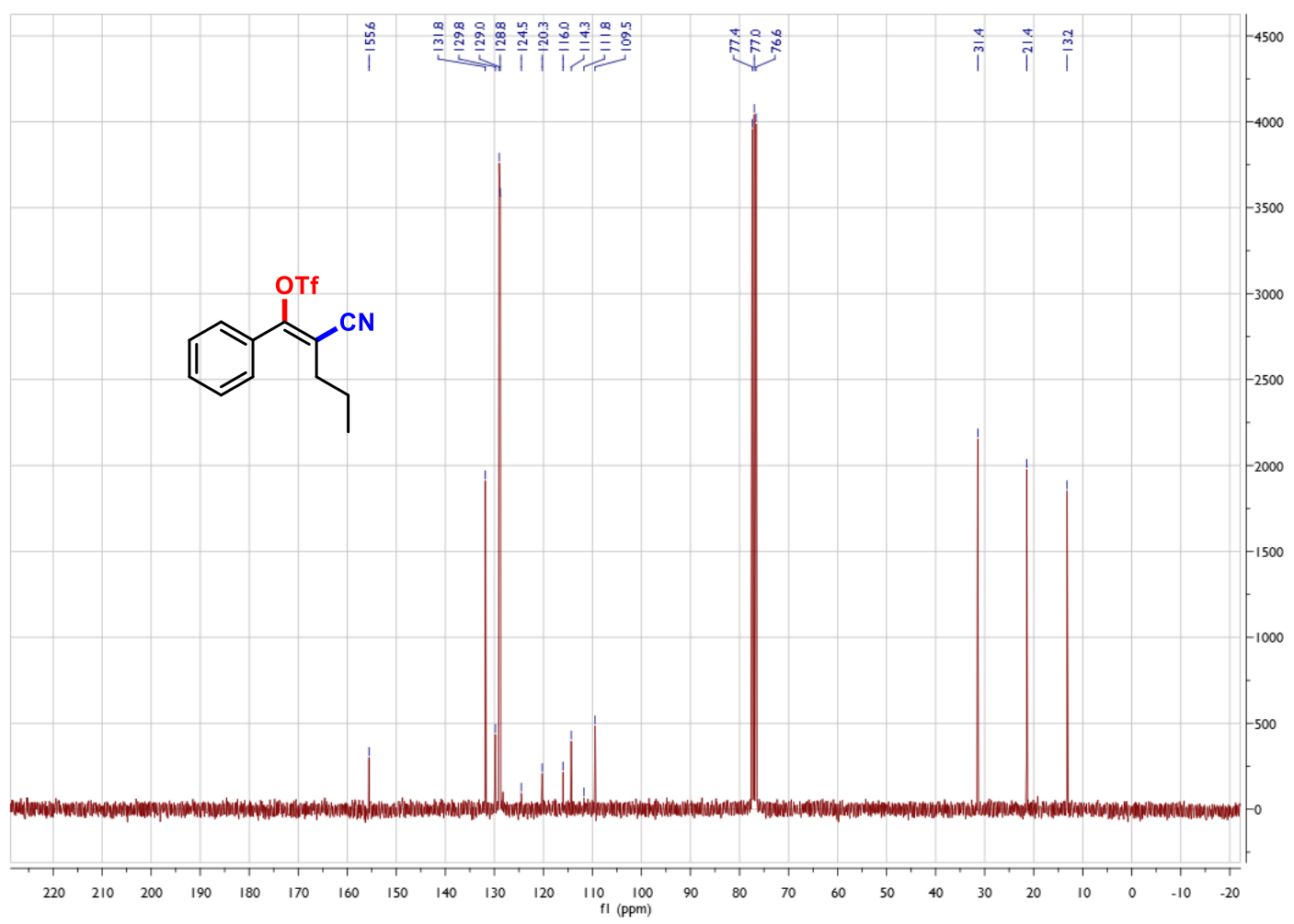


${ }^{19}$ F NMR Spectrum of (Z)-2-cyano-1-phenylpent-1-en-1-yl trifluoromethanesulfonate 2a

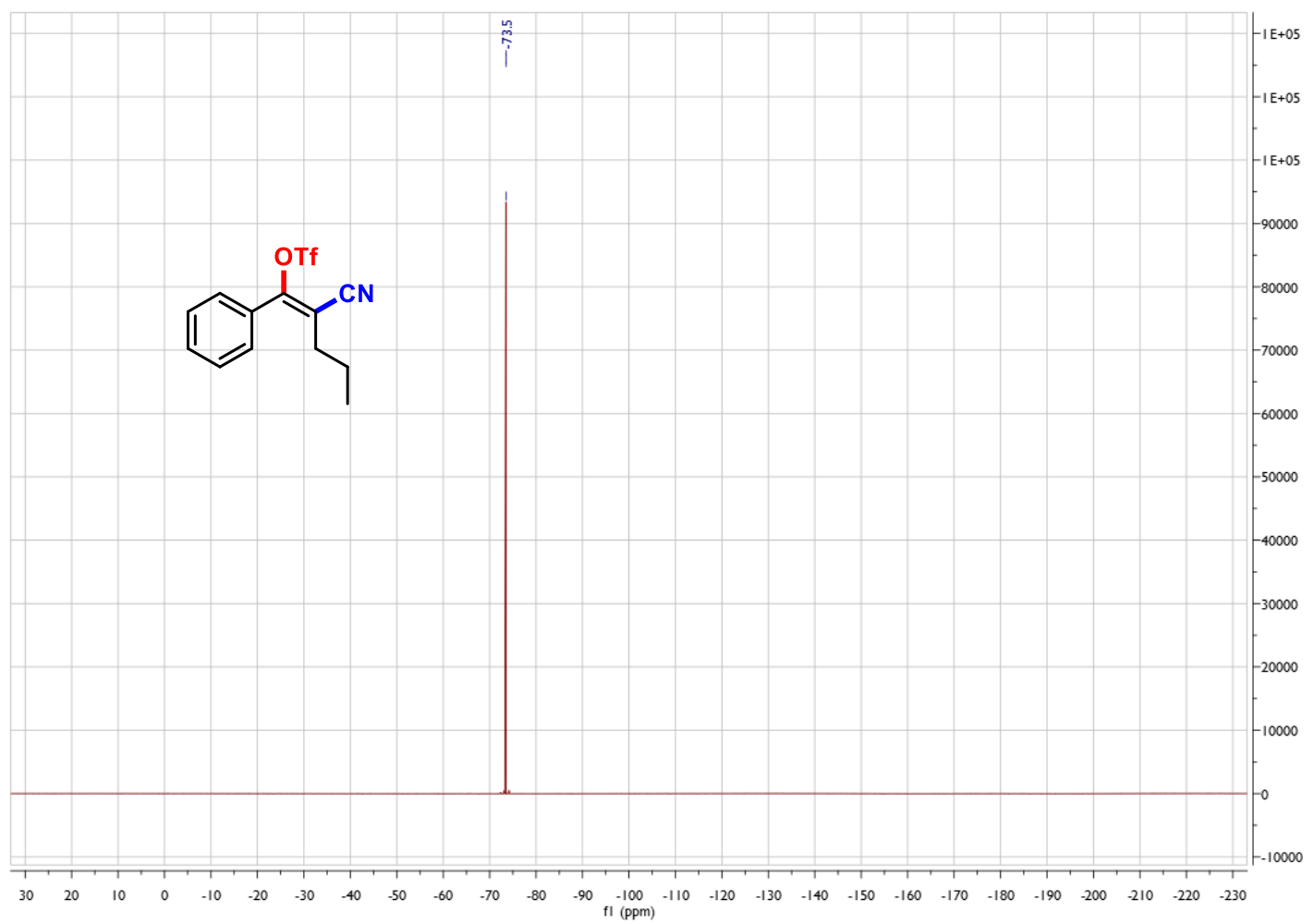

NOESY1D Spectrum of (Z)-2-cyano-1-phenylpent-1-en-1-yl trifluoromethanesulfonate 2a

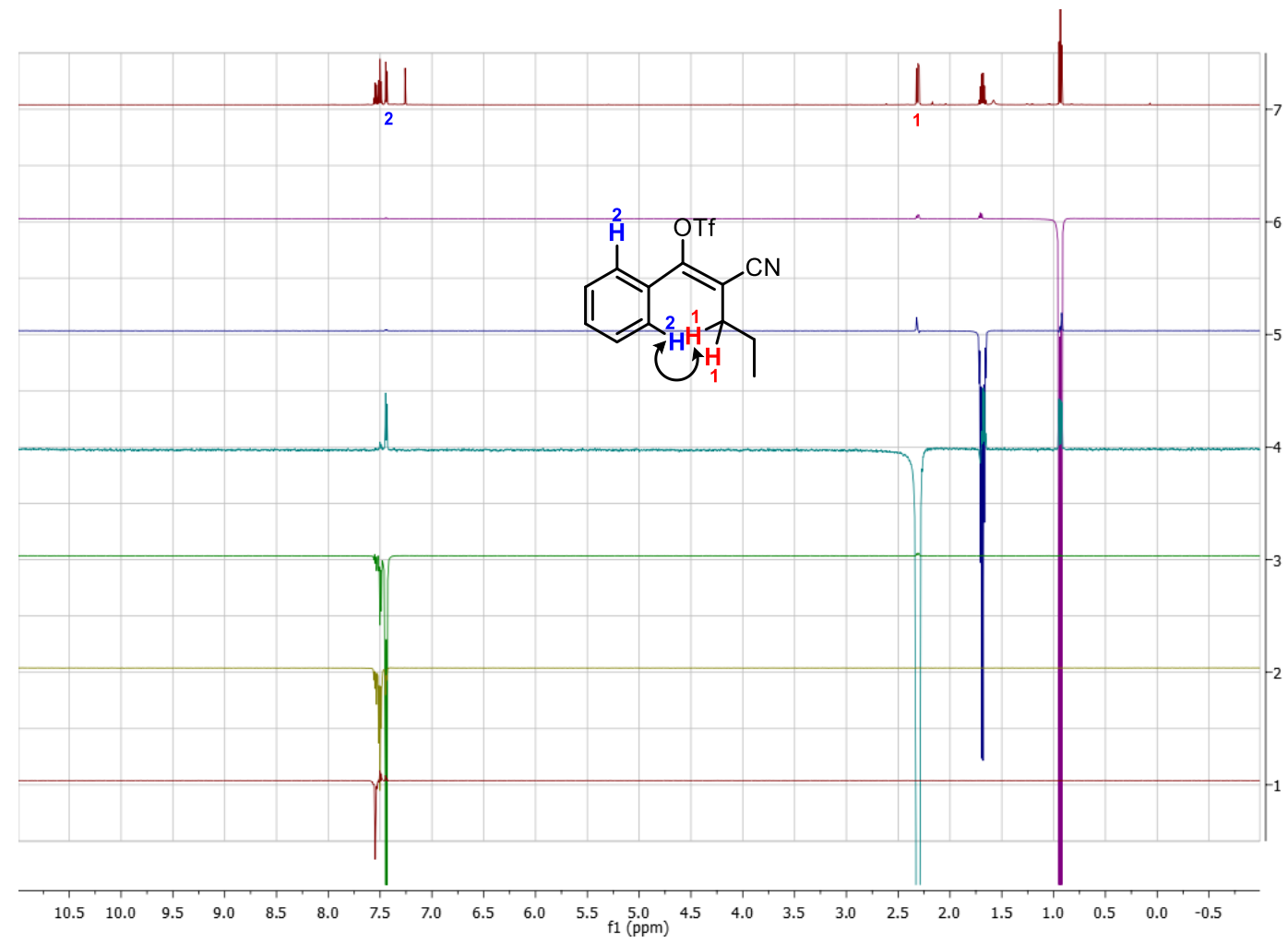


${ }^{1}$ H NMR Spectrum of (Z)-2-cyano-1-(p-tolyl)pent-1-en-1-yl trifluoromethanesulfonate $\mathbf{2 b}$

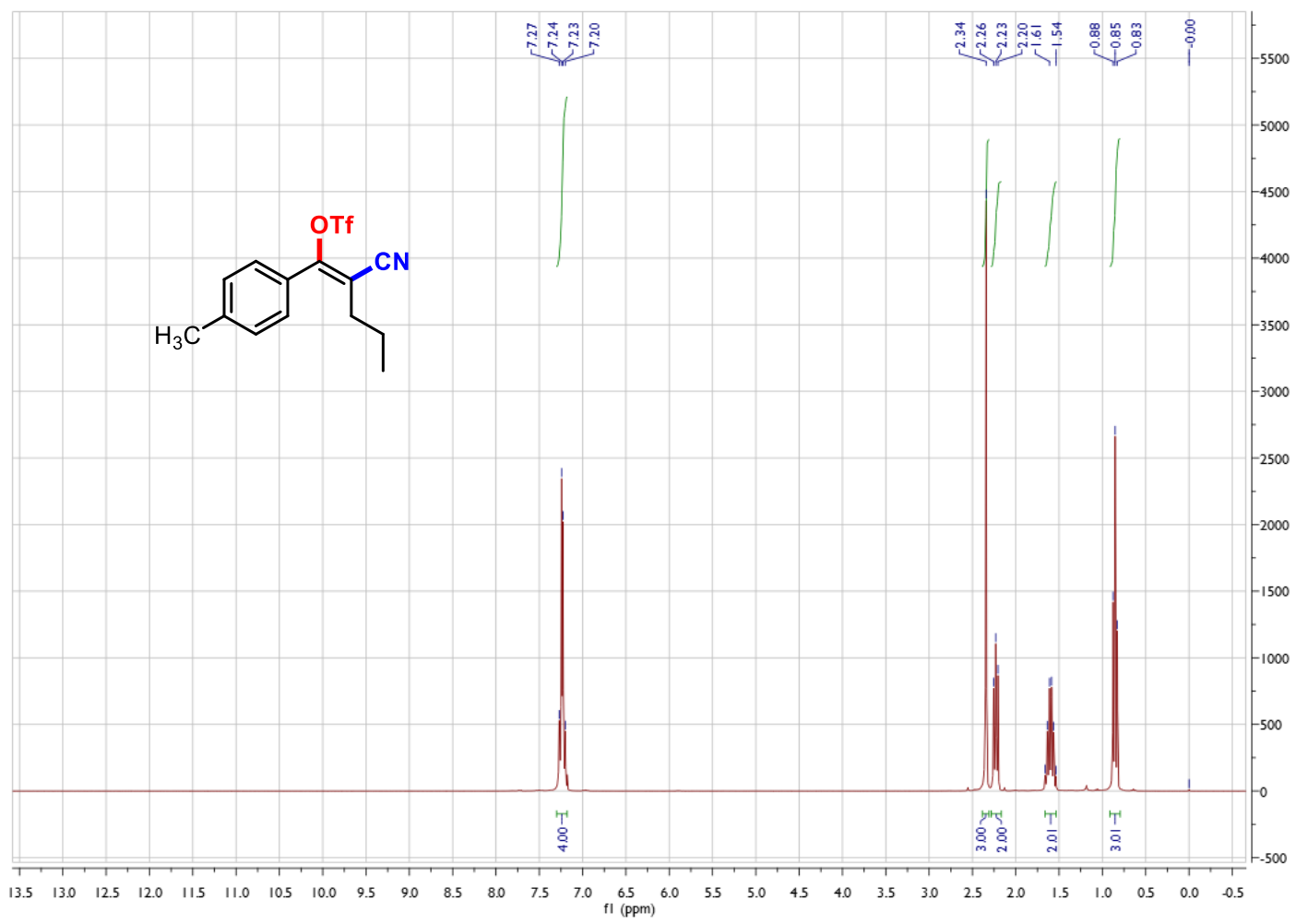

${ }^{13}$ C NMR Spectrum of (Z)-2-cyano-1-(p-tolyl)pent-1-en-1-yl trifluoromethanesulfonate $2 \mathrm{~b}$

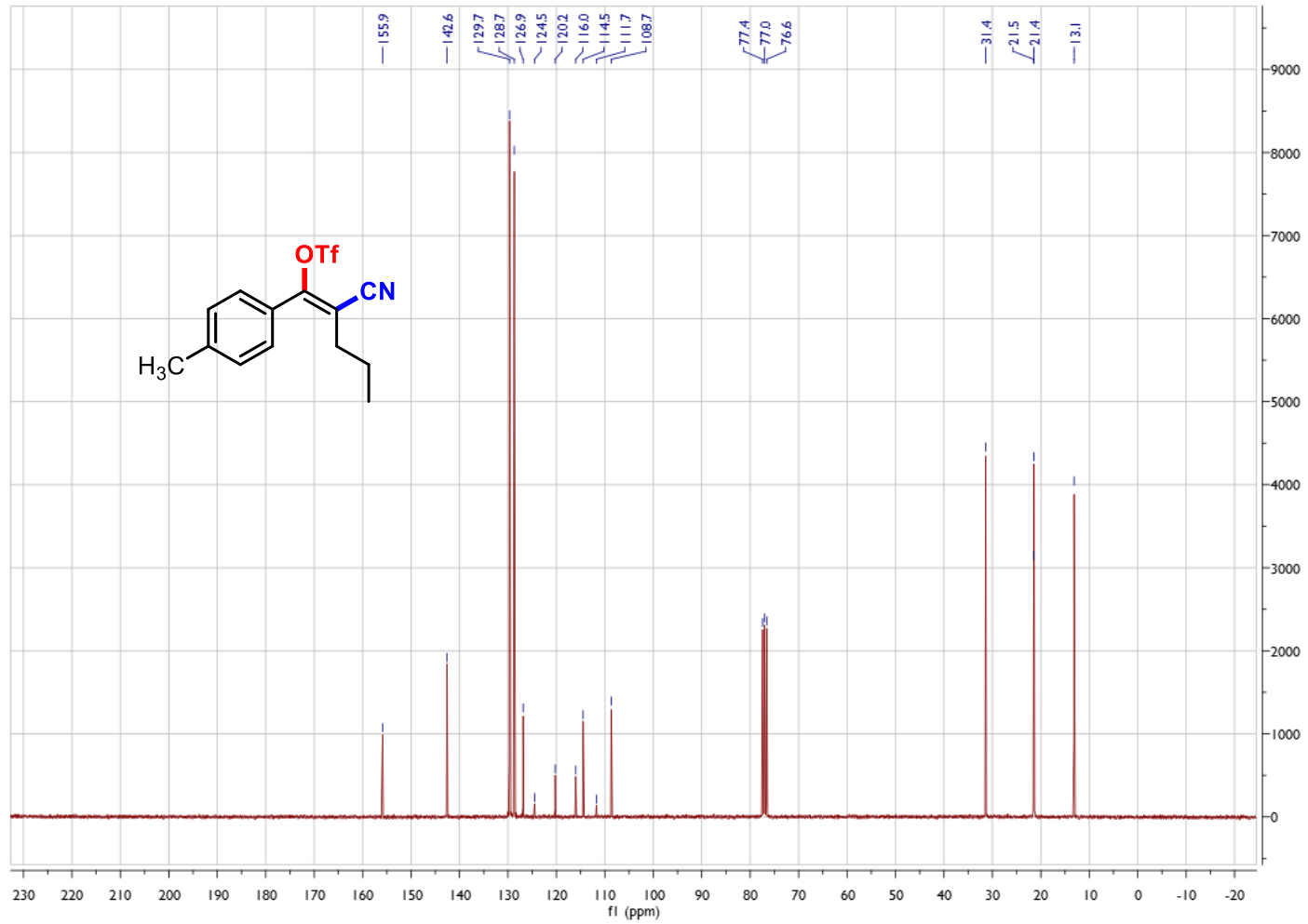


${ }^{19}$ F NMR Spectrum of (Z)-2-cyano-1-( $p$-tolyl)pent-1-en-1-yl trifluoromethanesulfonate $2 \mathrm{~b}$

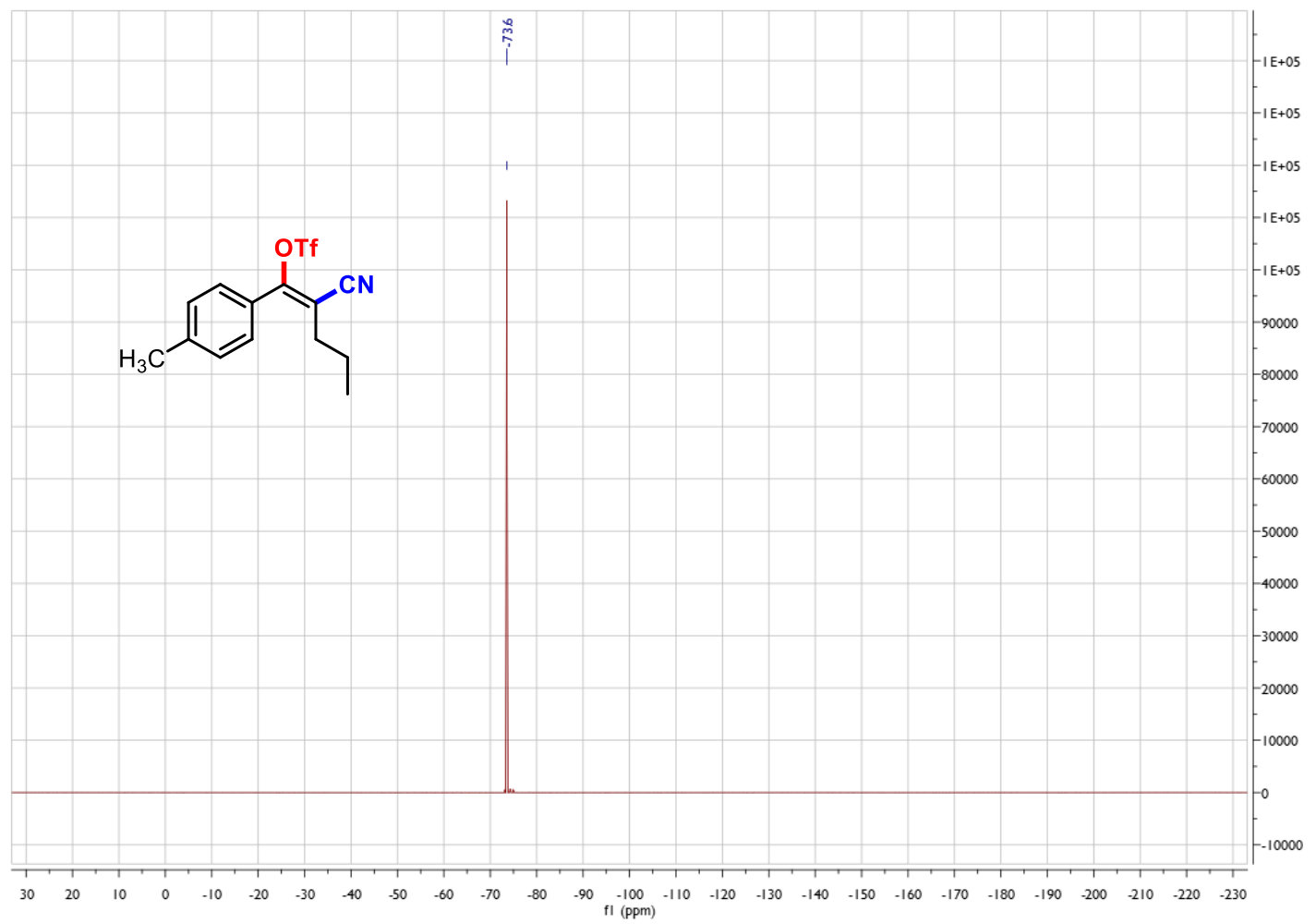


${ }^{1}$ H NMR Spectrum of (Z)-1-(4-chlorophenyl)-2-cyanopent-1-en-1-yl trifluoromethanesulfonate $2 \mathrm{c}$

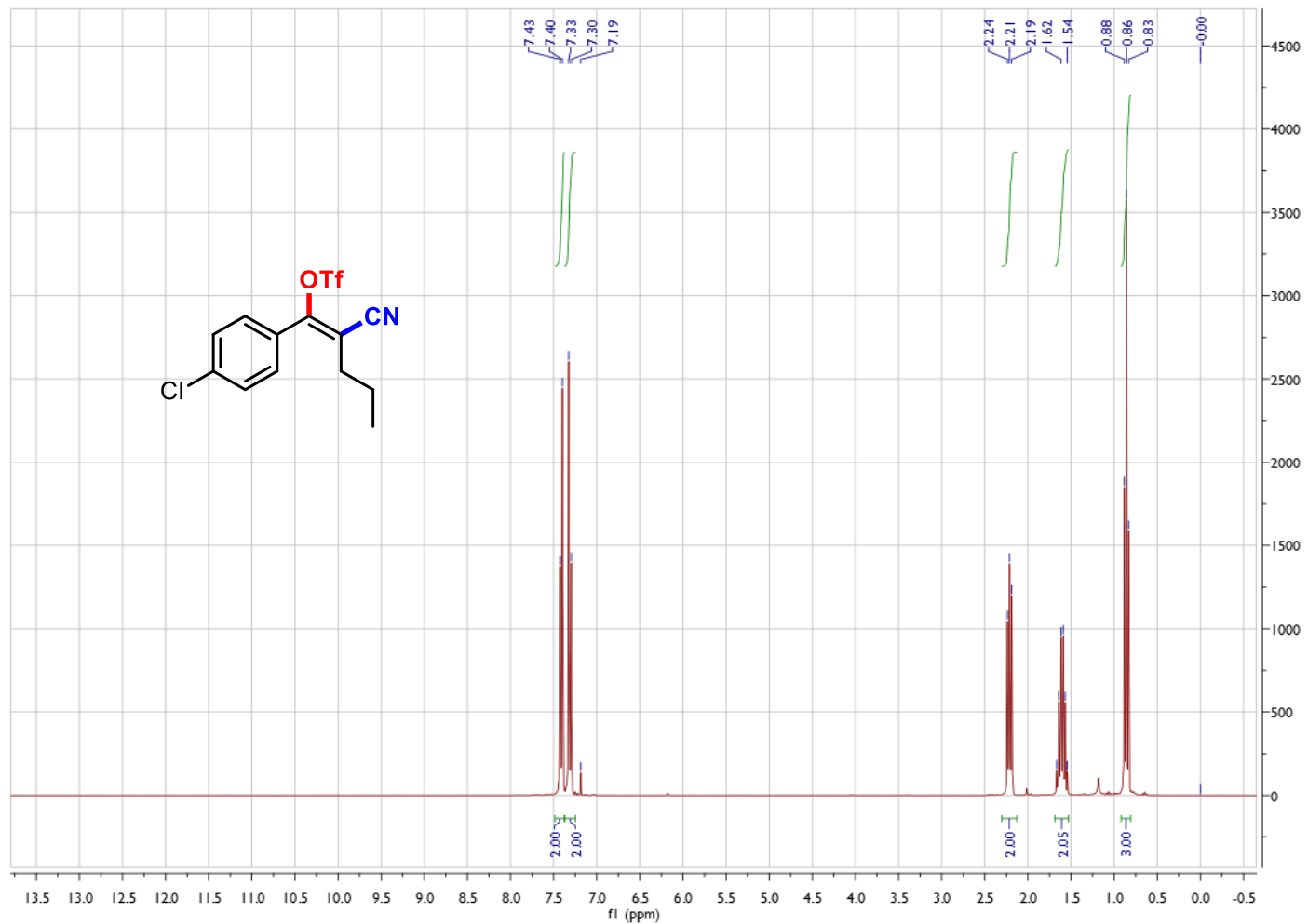

${ }^{13}$ C NMR Spectrum of (Z)-1-(4-chlorophenyl)-2-cyanopent-1-en-1-yl trifluoromethanesulfonate $2 \mathrm{c}$

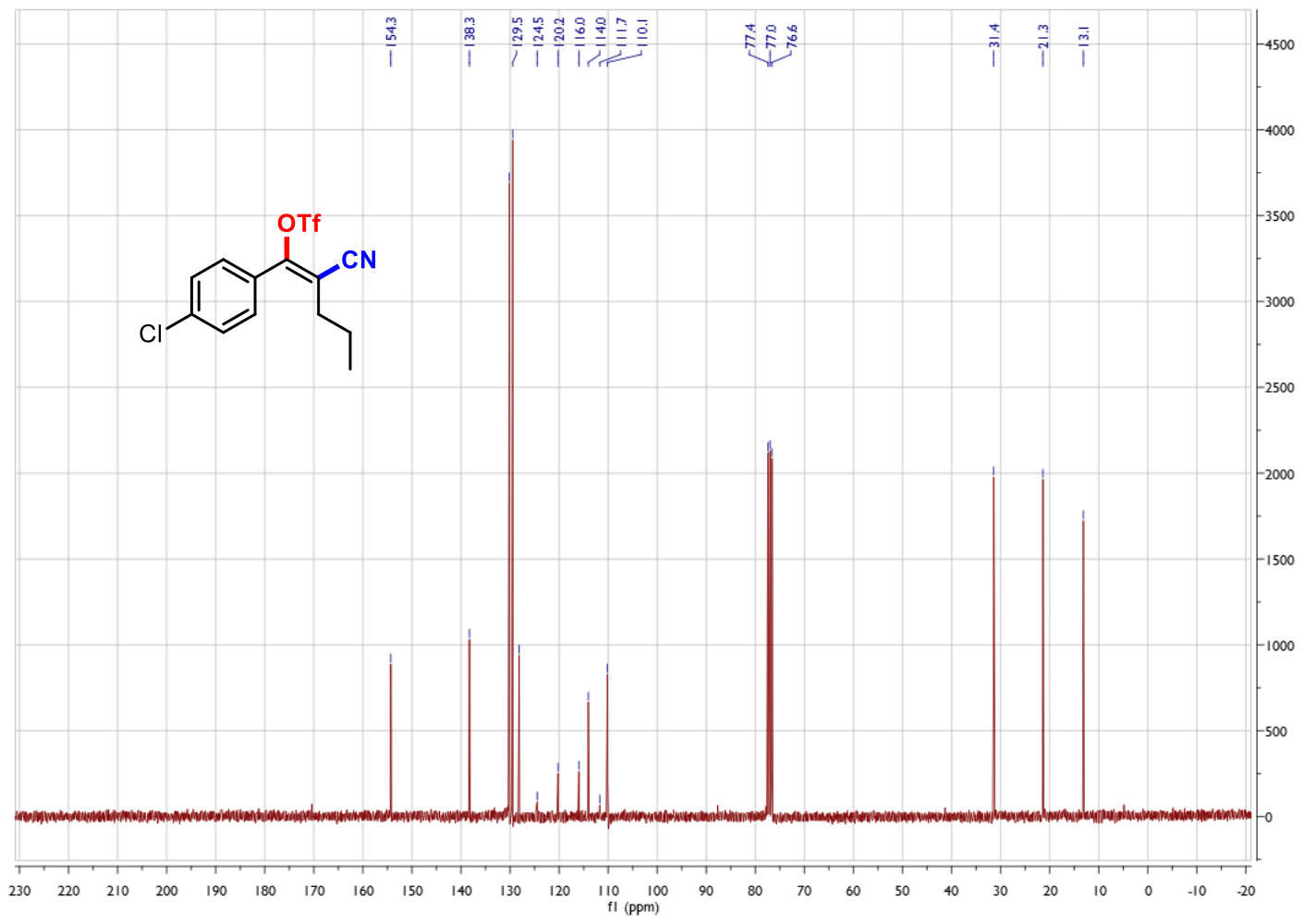


${ }^{19}$ F NMR Spectrum of (Z)-1-(4-chlorophenyl)-2-cyanopent-1-en-1-yl trifluoromethanesulfonate $2 \mathrm{c}$

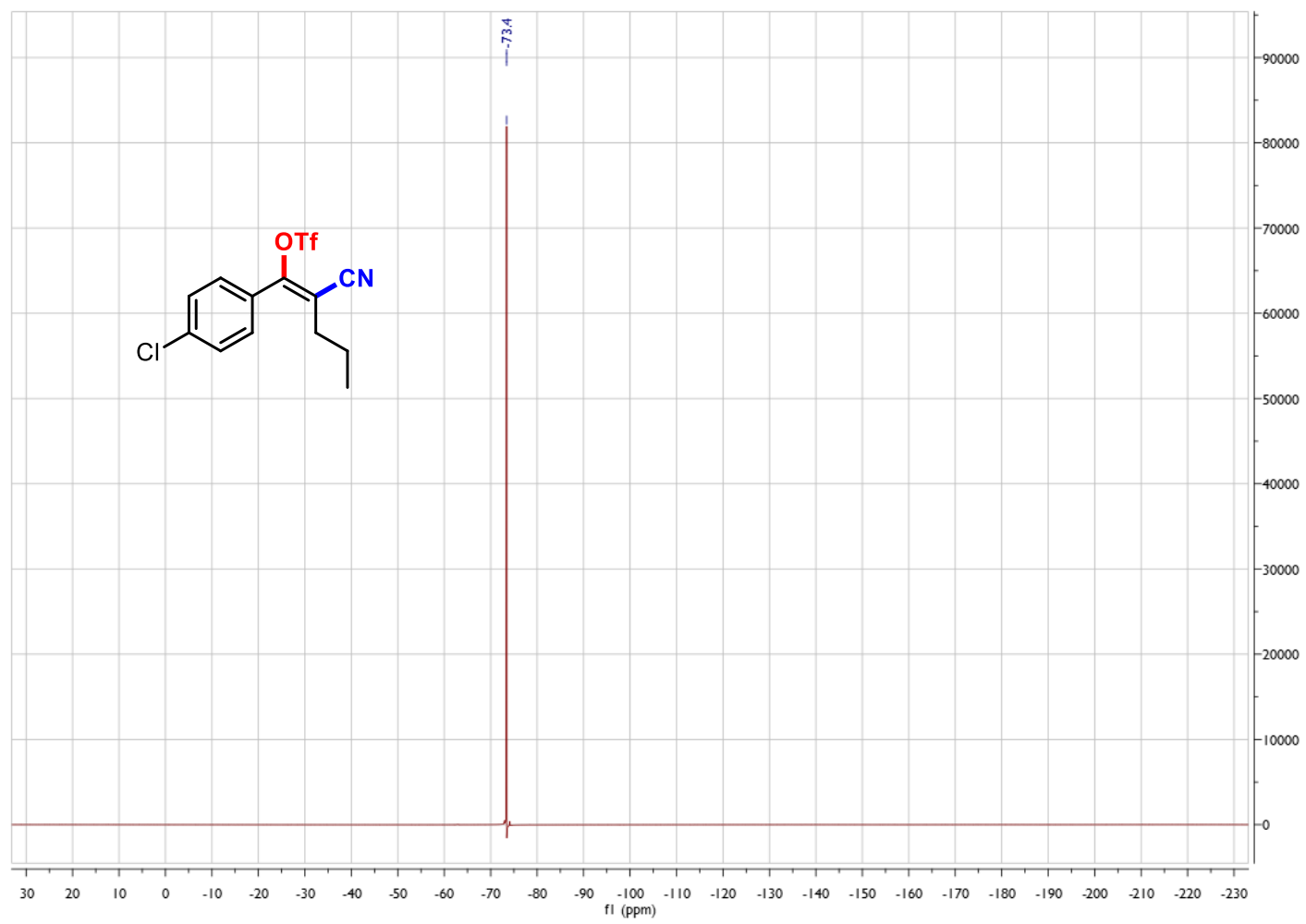


${ }^{1} \mathrm{H}$ NMR Spectrum of (Z)-1-(4-chloro-3-methylphenyl)-2-cyanopent-1-en-1-yl trifluoromethanesulfonate $2 \mathrm{~d}$

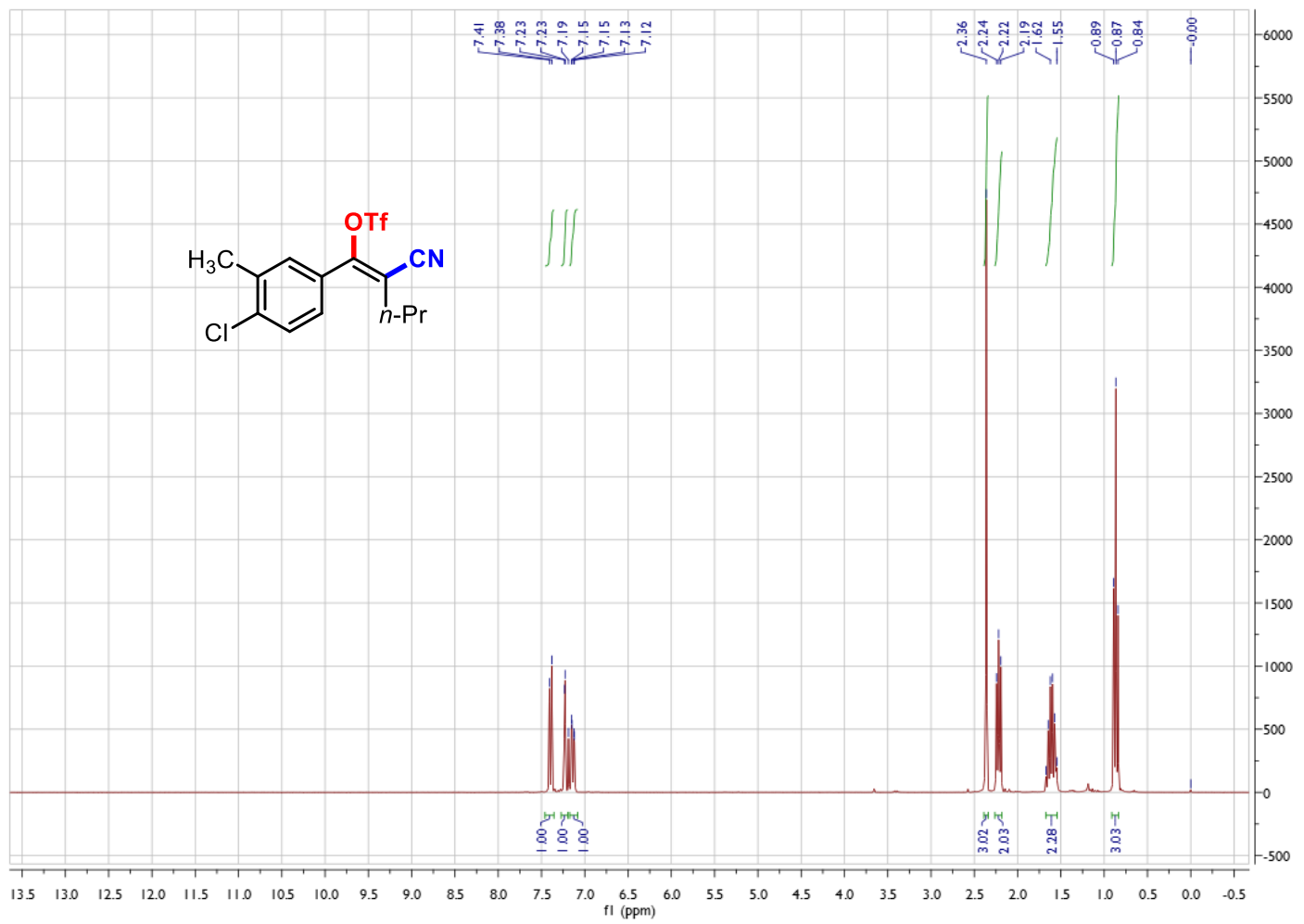

${ }^{13}$ C NMR Spectrum of (Z)-1-(4-chloro-3-methylphenyl)-2-cyanopent-1-en-1-yl trifluoromethanesulfonate $2 \mathrm{~d}$

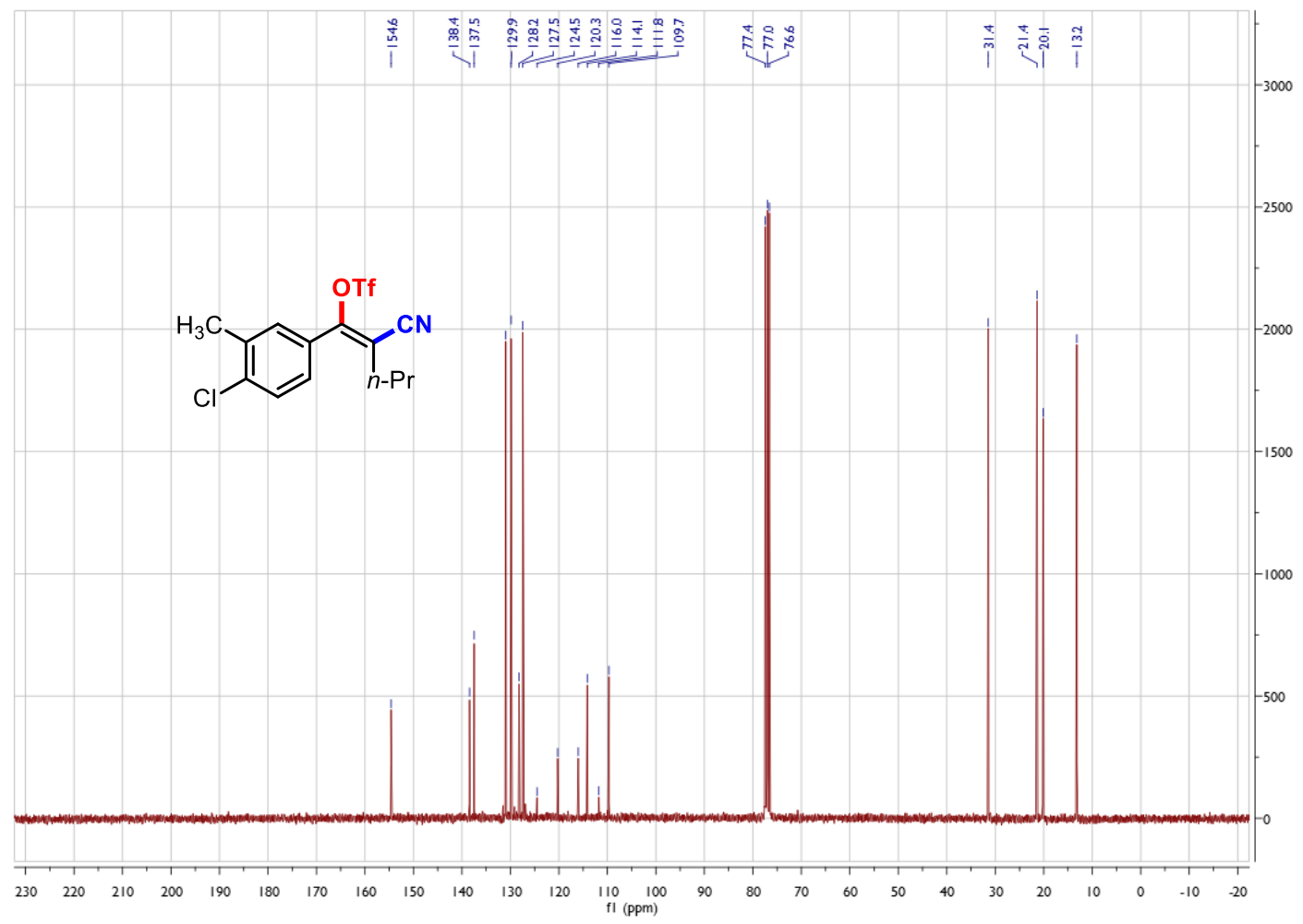


${ }^{19}$ F NMR Spectrum of (Z)-1-(4-chloro-3-methylphenyl)-2-cyanopent-1-en-1-yl trifluoromethanesulfonate $2 \mathrm{~d}$

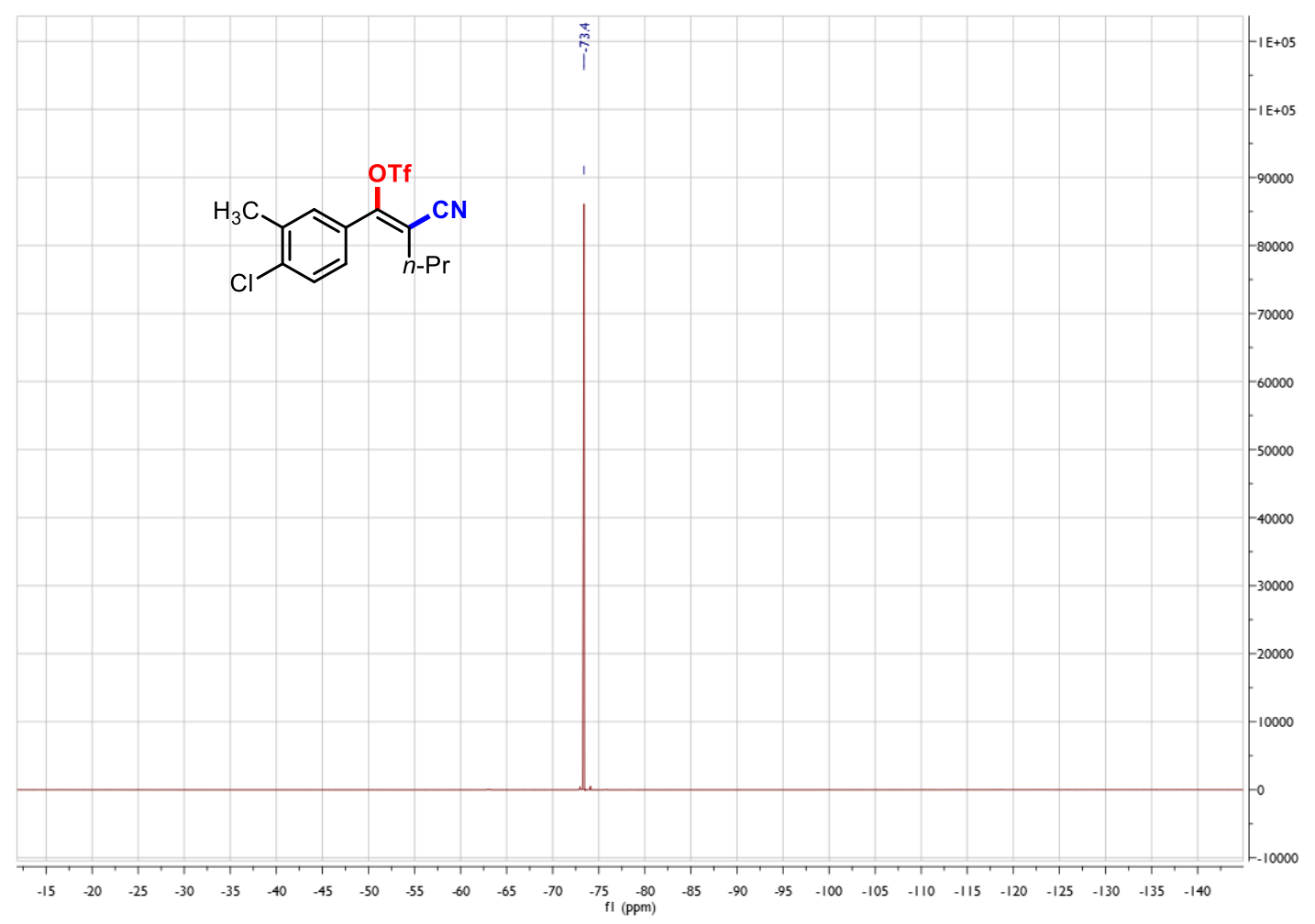


${ }^{1}$ H NMR Spectrum of (Z)-1-(4-bromophenyl)-2-cyanopent-1-en-1-yl trifluoro methanesulfonate $2 \mathrm{e}$

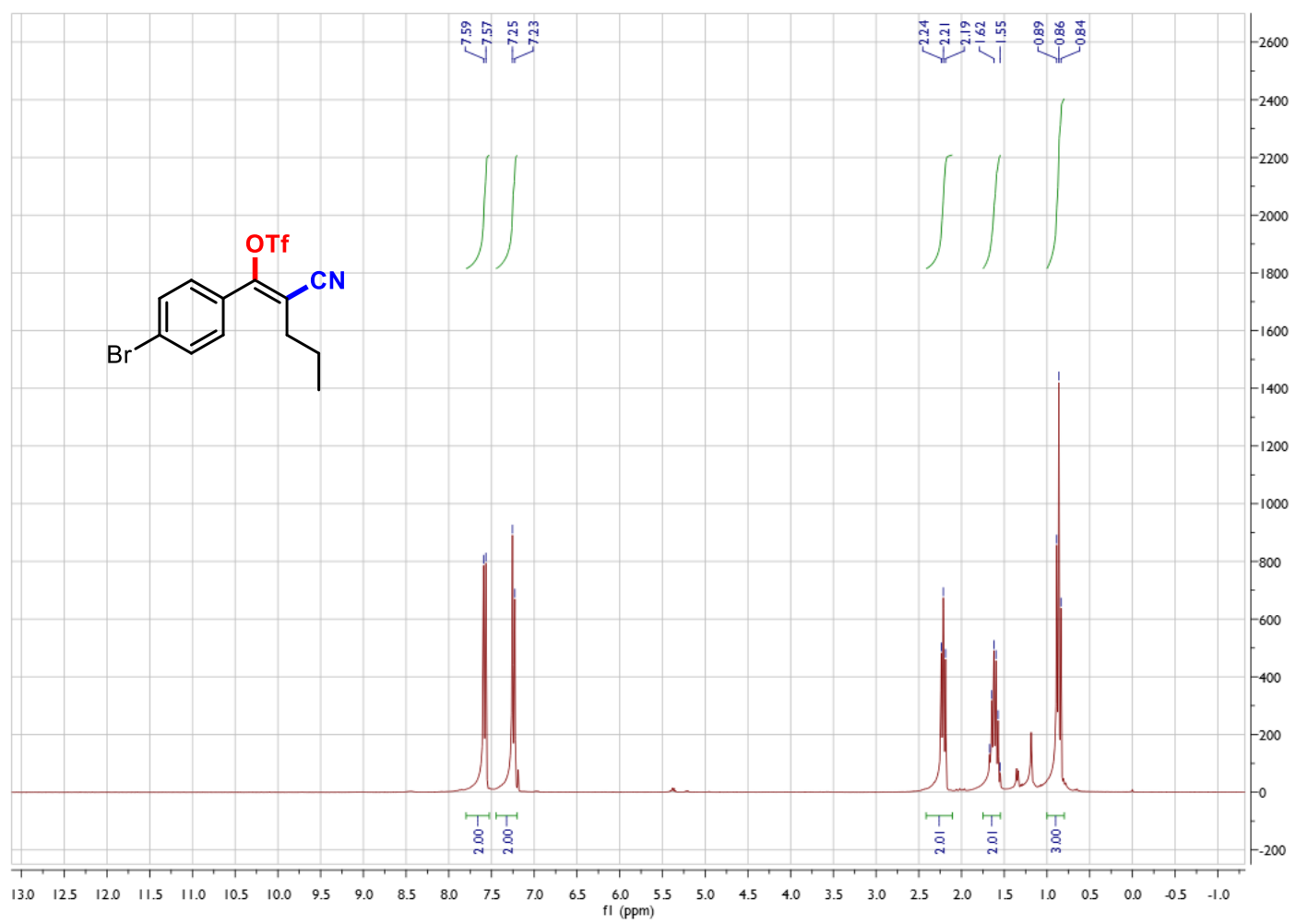

${ }^{13}$ C NMR Spectrum of (Z)-1-(4-bromophenyl)-2-cyanopent-1-en-1-yl trifluoro methanesulfonate $2 \mathrm{e}$

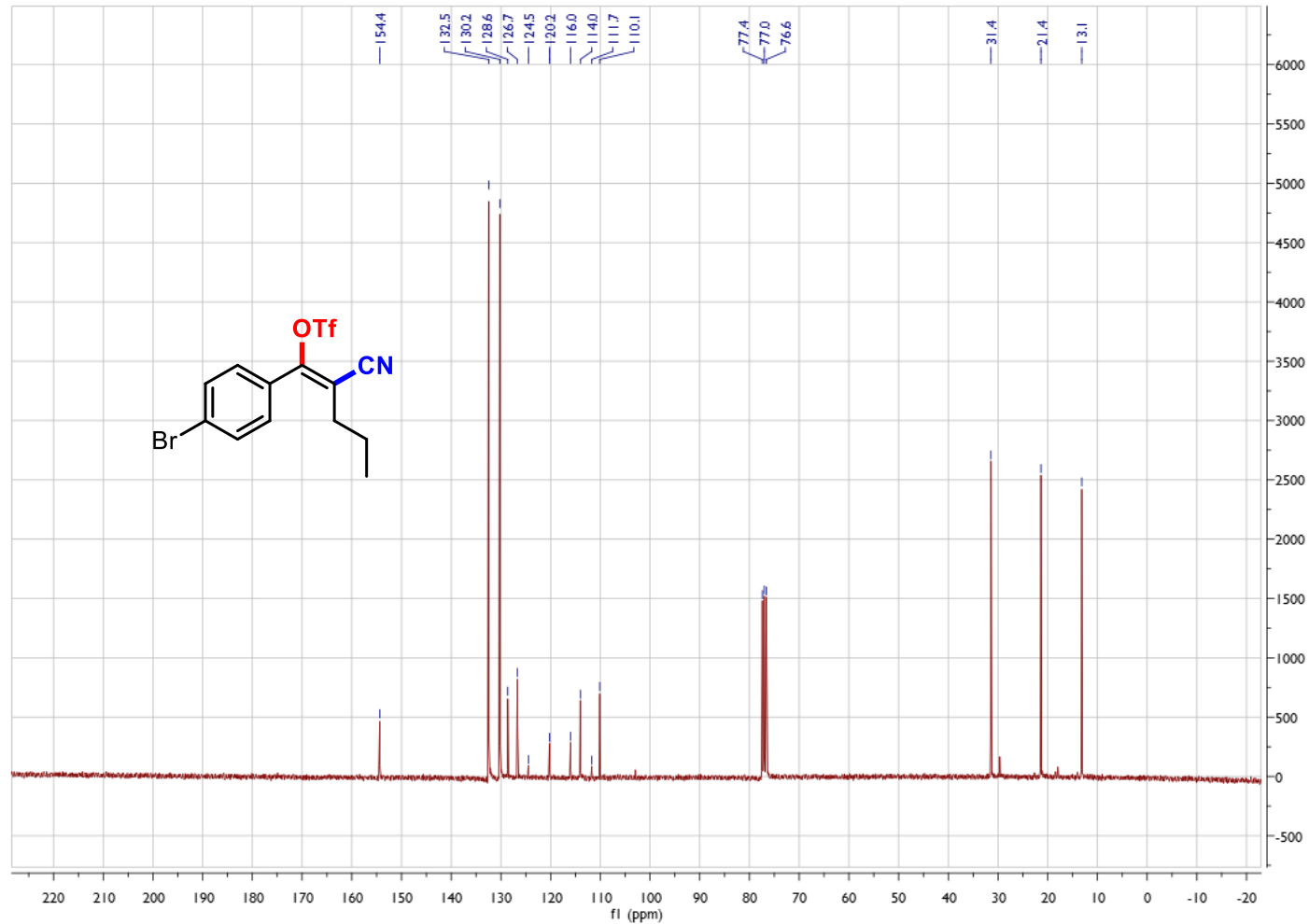


${ }^{19}$ F NMR Spectrum of (Z)-1-(4-bromophenyl)-2-cyanopent-1-en-1-yl trifluoro methanesulfonate $2 \mathrm{e}$

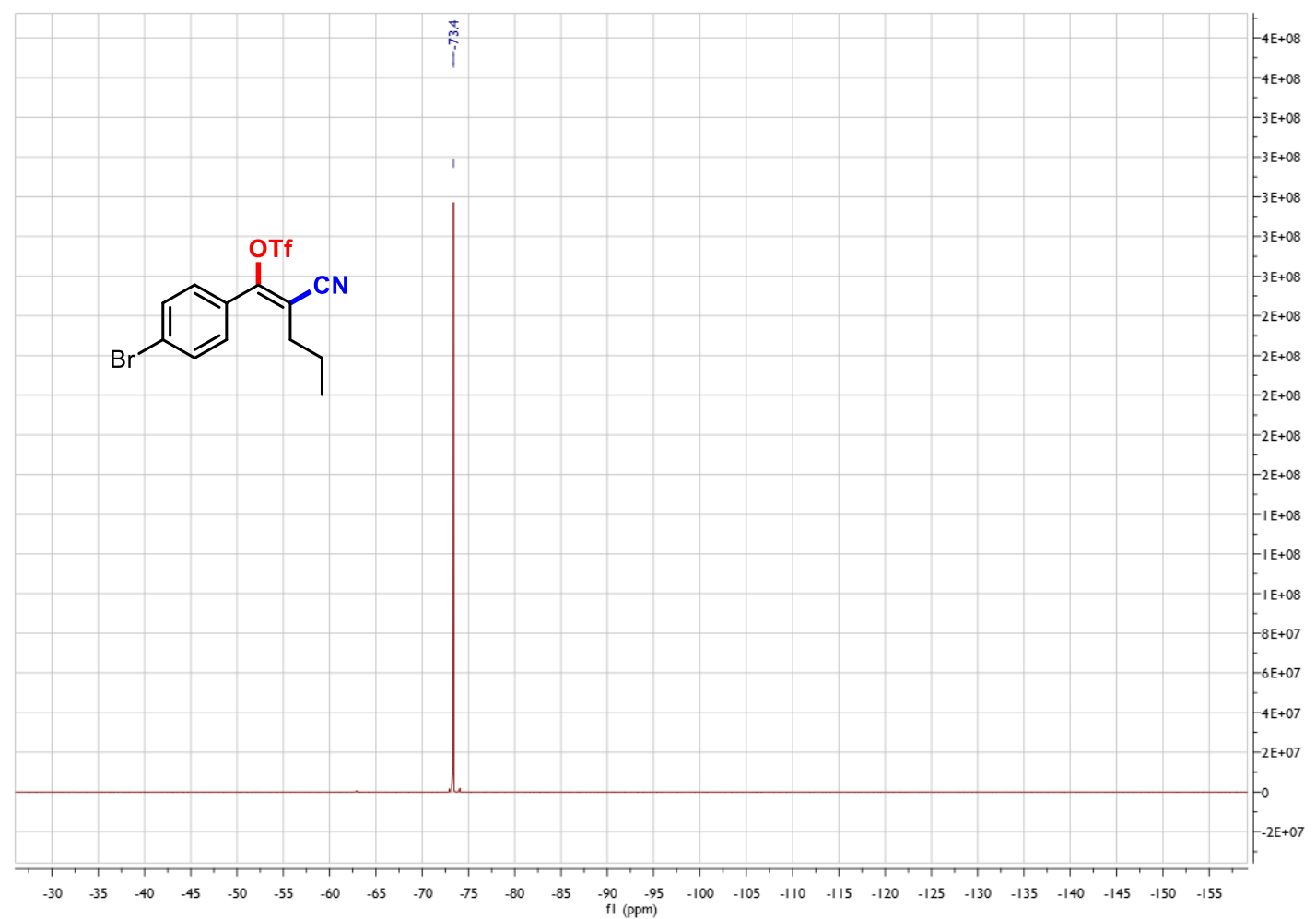


${ }^{1}$ H NMR Spectrum of (Z)-2-cyano-1-(4-fluorophenyl)pent-1-en-1-yl trifluoro methanesulfonate $2 \mathrm{f}$

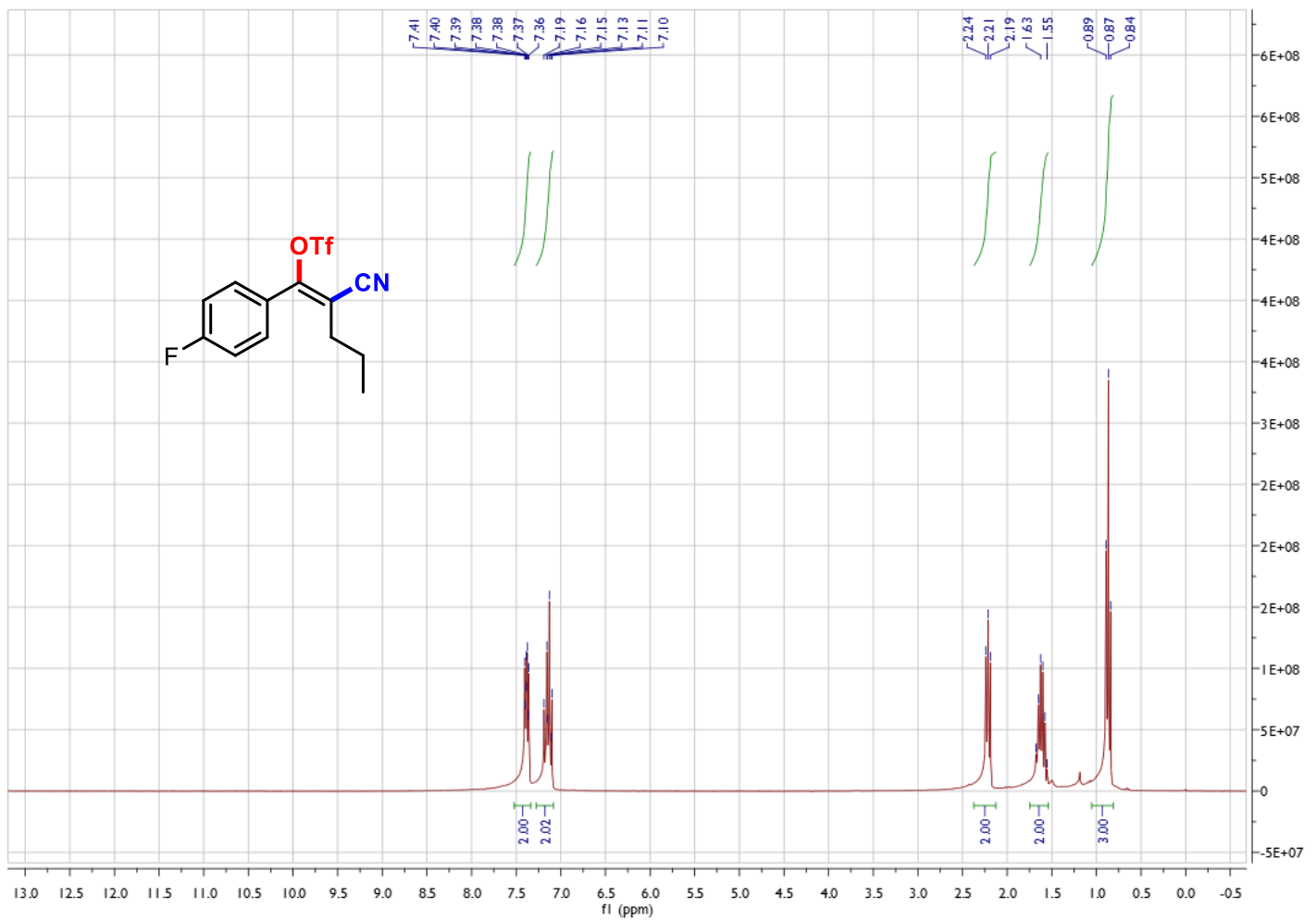

${ }^{13}$ C NMR Spectrum of (Z)-2-cyano-1-(4-fluorophenyl)pent-1-en-1-yl trifluoro methanesulfonate $2 f$

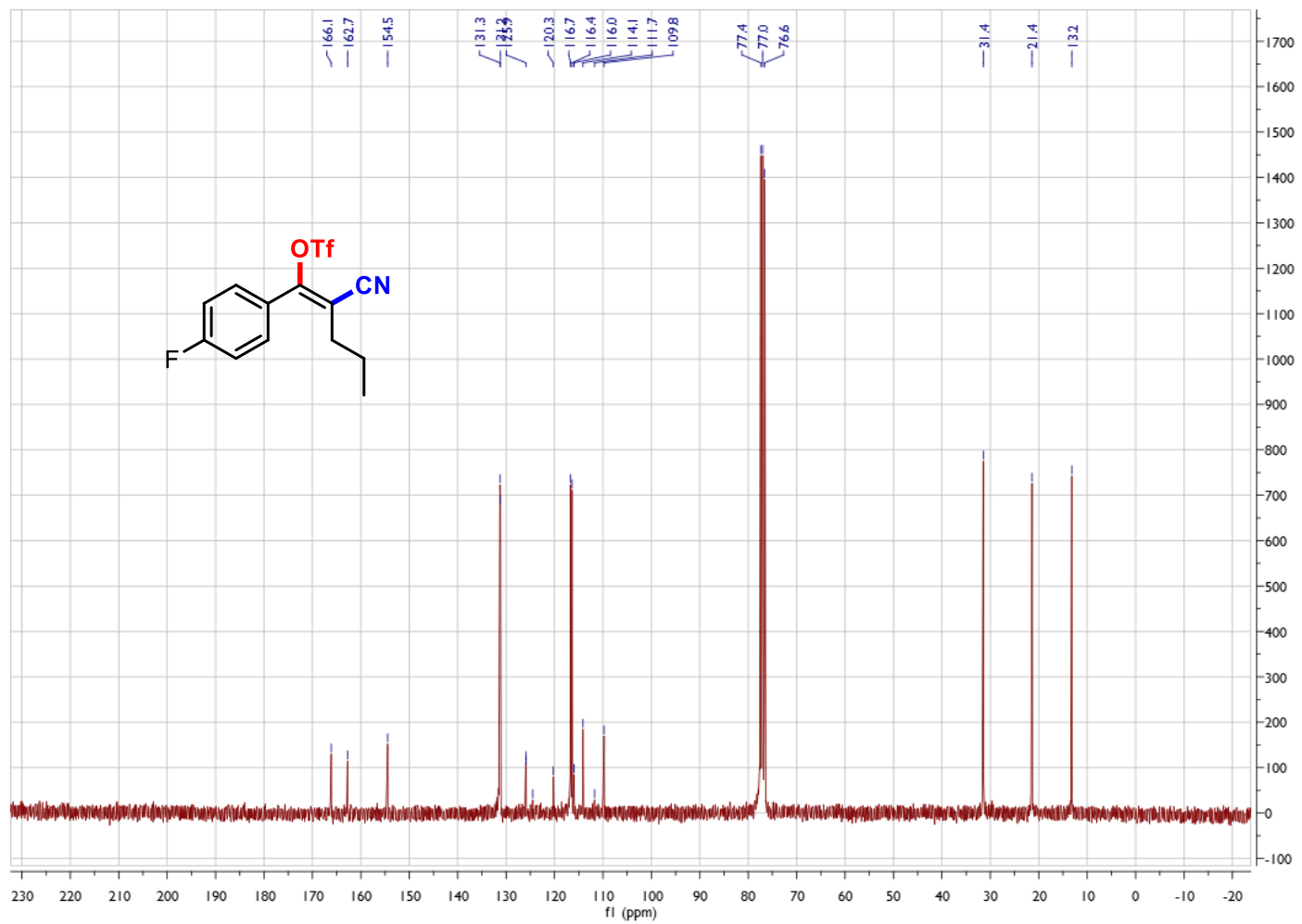


${ }^{19}$ F NMR Spectrum of (Z)-2-cyano-1-(4-fluorophenyl)pent-1-en-1-yl trifluoro methanesulfonate $2 f$

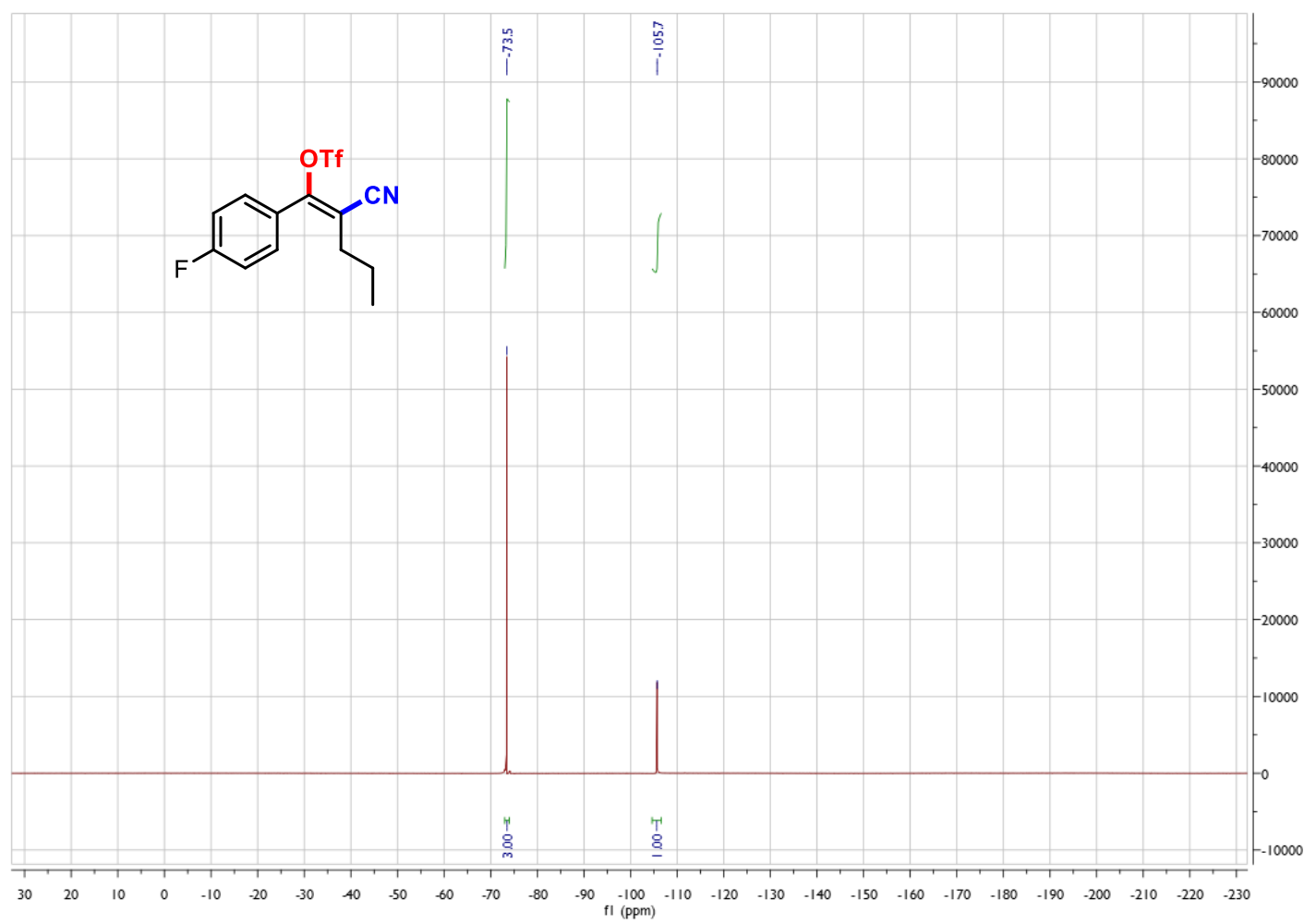


${ }^{1}$ H NMR Spectrum of (Z)-4-(2-cyano-1-(((trifluoromethyl)sulfonyl)oxy)pent-1-en1-yl)phenyl acetate $2 \mathrm{~g}$

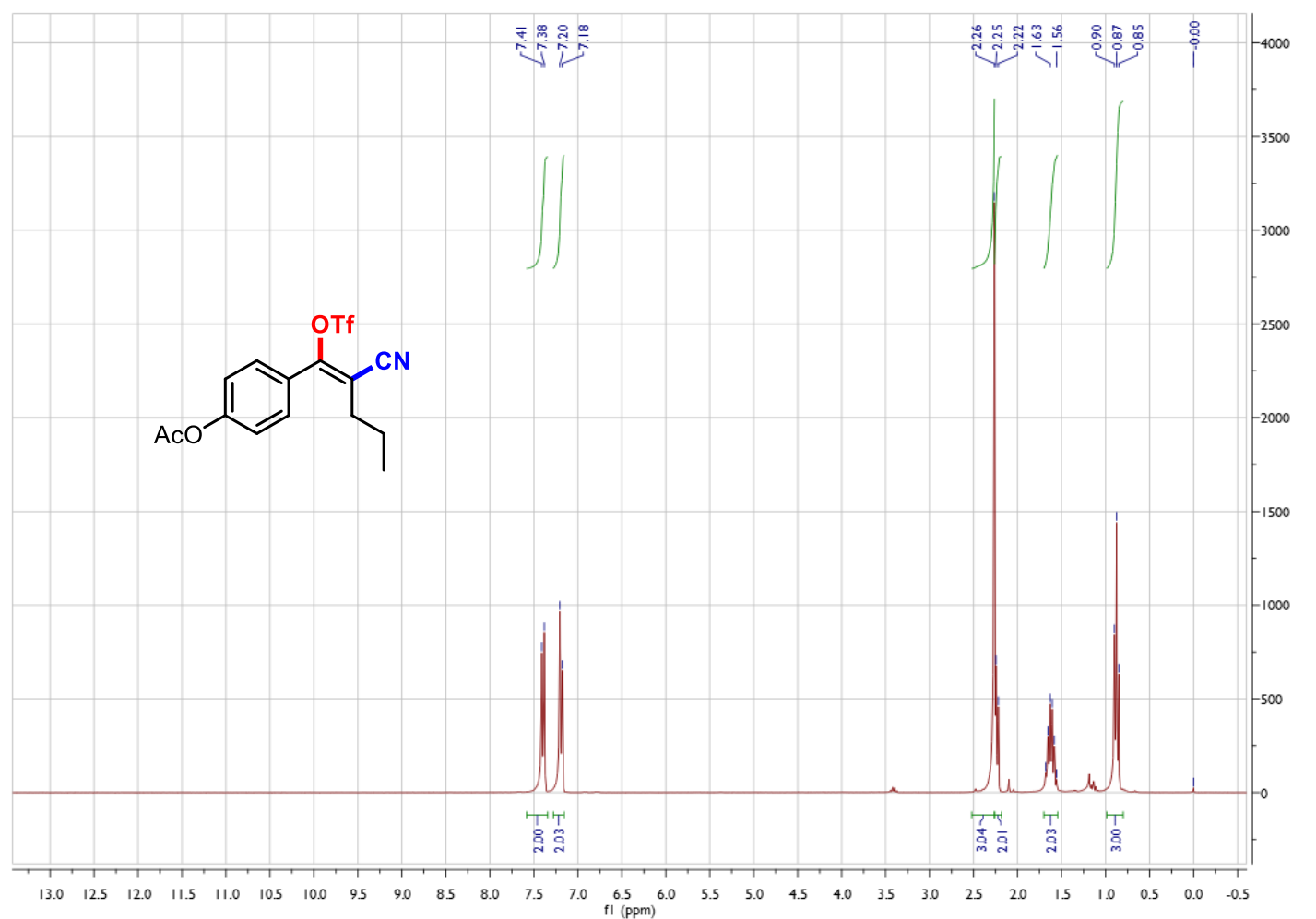

${ }^{13}$ C NMR Spectrum of (Z)-4-(2-cyano-1-(((trifluoromethyl)sulfonyl)oxy)pent-1en-1-yl)phenyl acetate $2 \mathrm{~g}$

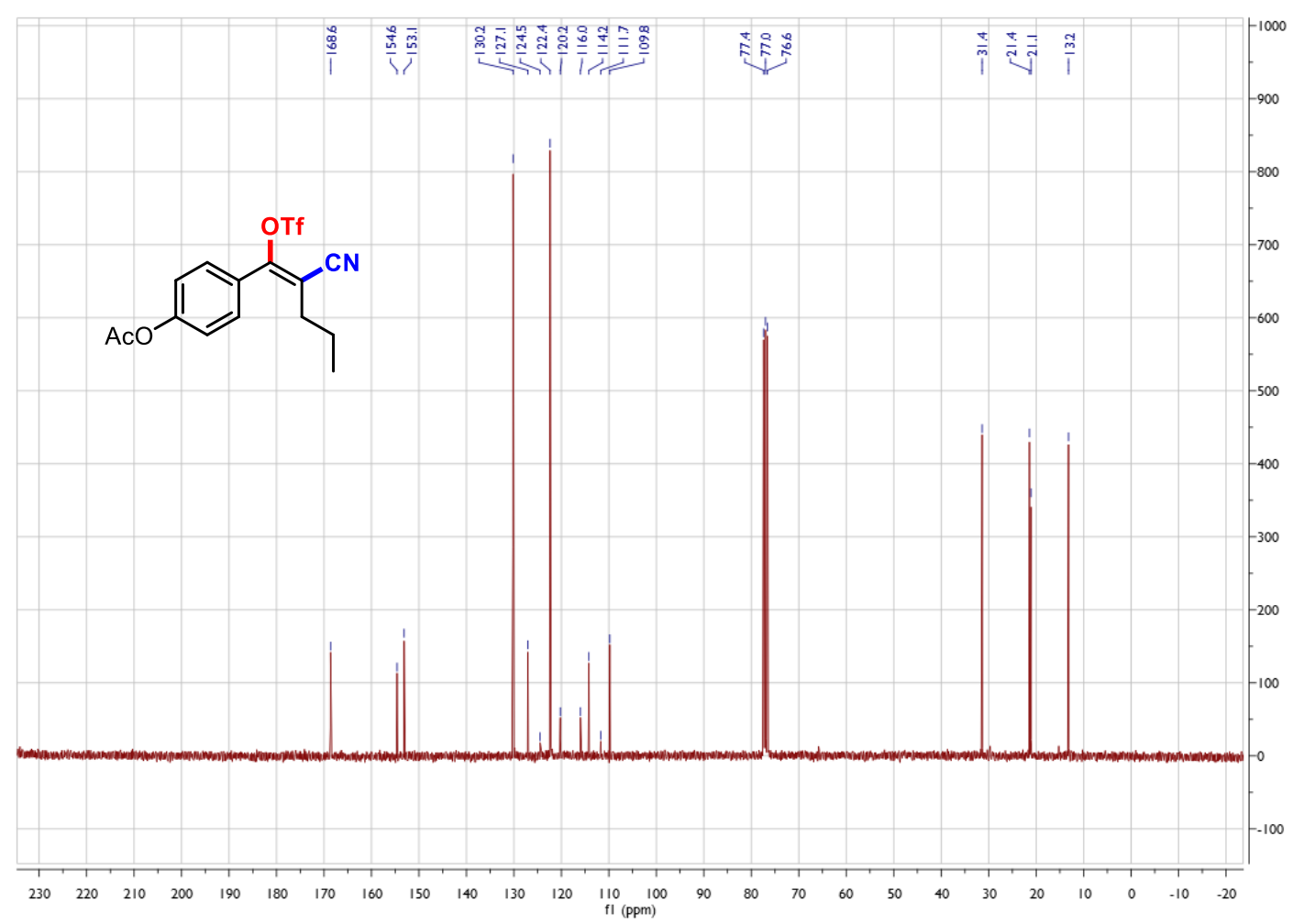


${ }^{19}$ F NMR Spectrum of (Z)-4-(2-cyano-1-(((trifluoromethyl)sulfonyl)oxy)pent-1-en1-yl)phenyl acetate $2 \mathrm{~g}$

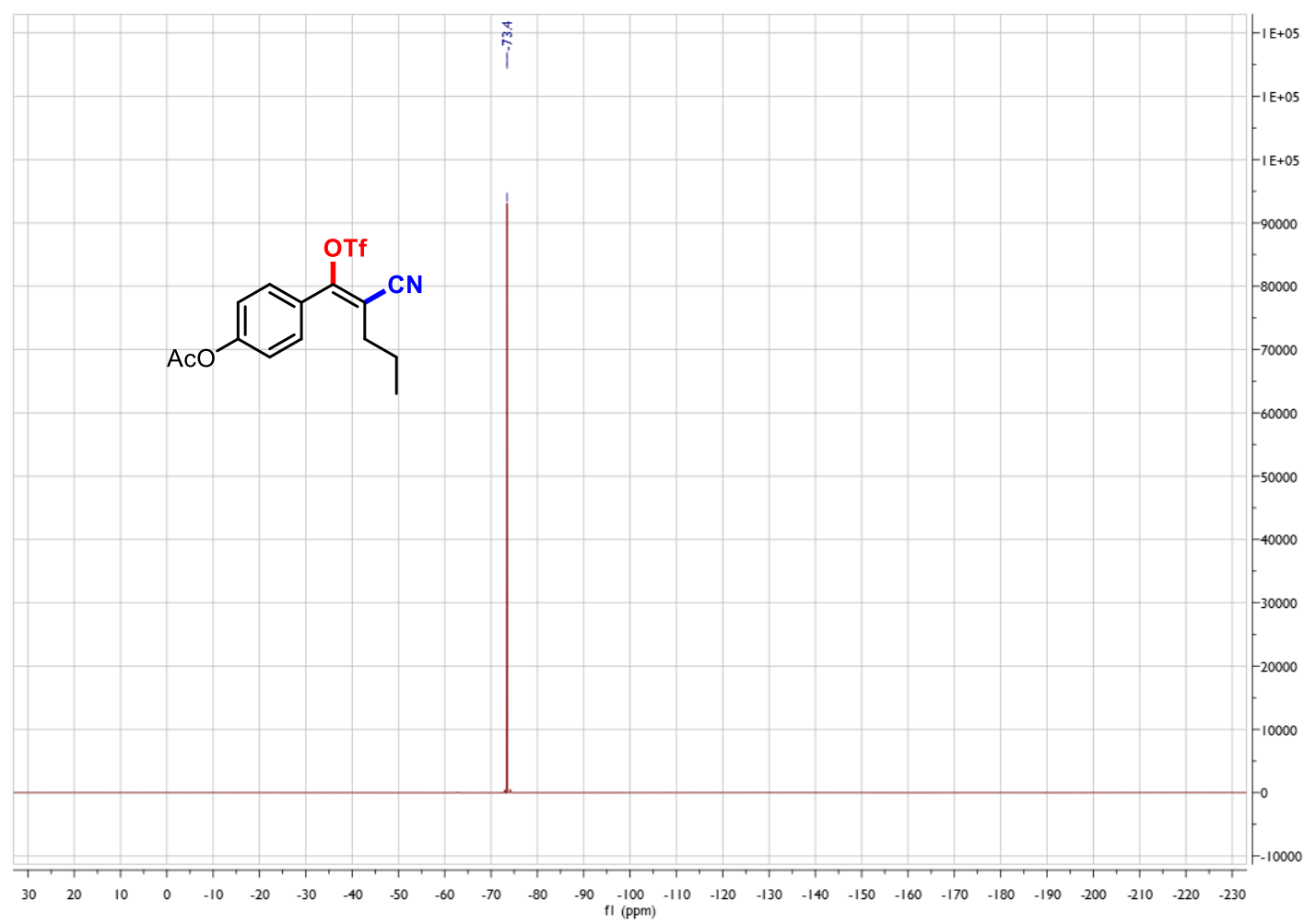


${ }^{1}$ H NMR Spectrum of (Z)-2-cyano-1-(4-(1,3-dioxoisoindolin-2-yl)phenyl)pent-1en-1-yl trifluoromethanesulfonate $2 h$

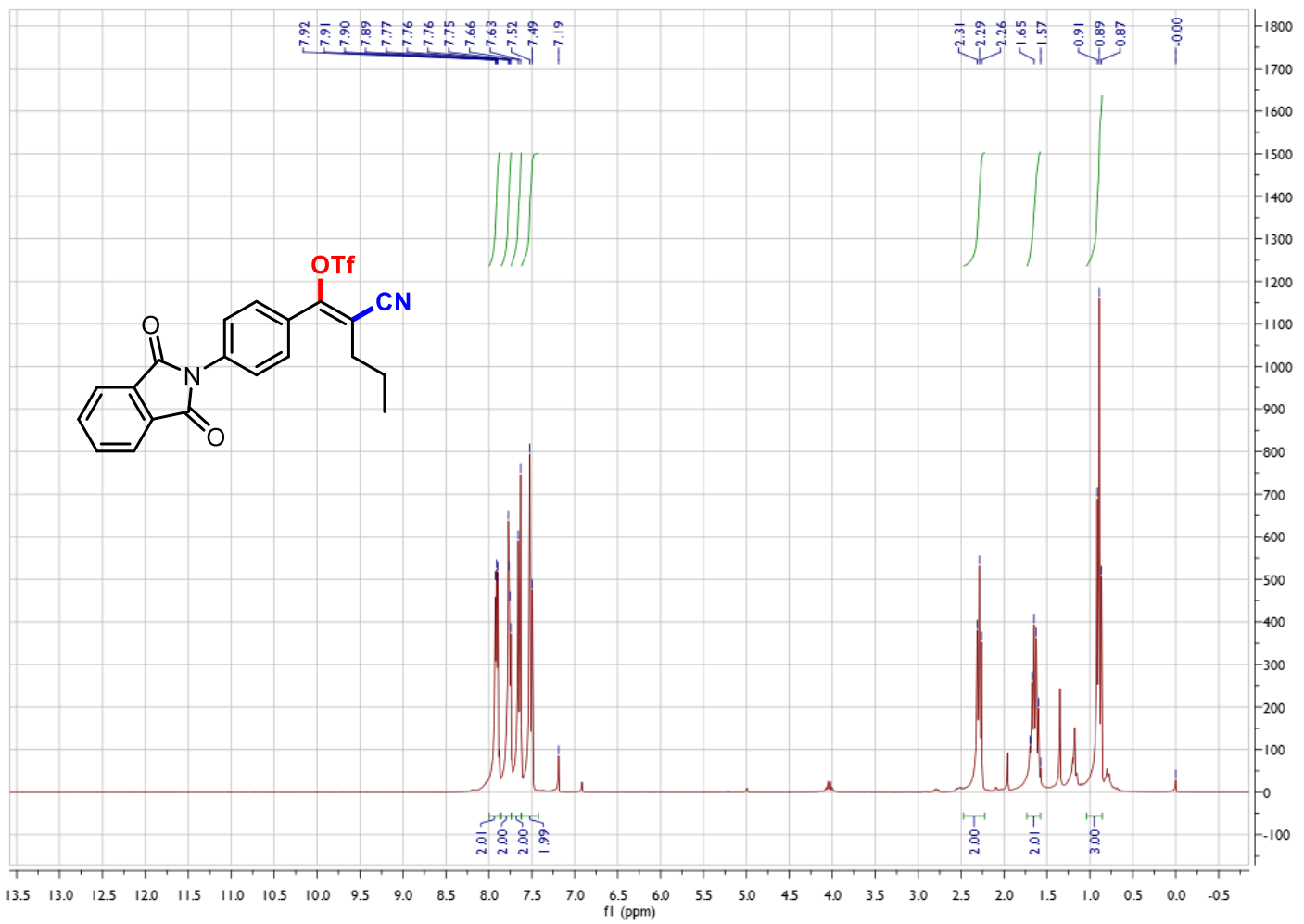

${ }^{13}$ C NMR Spectrum of (Z)-2-cyano-1-(4-(1,3-dioxoisoindolin-2-yl)phenyl)pent-1en-1-yl trifluoromethanesulfonate $2 h$

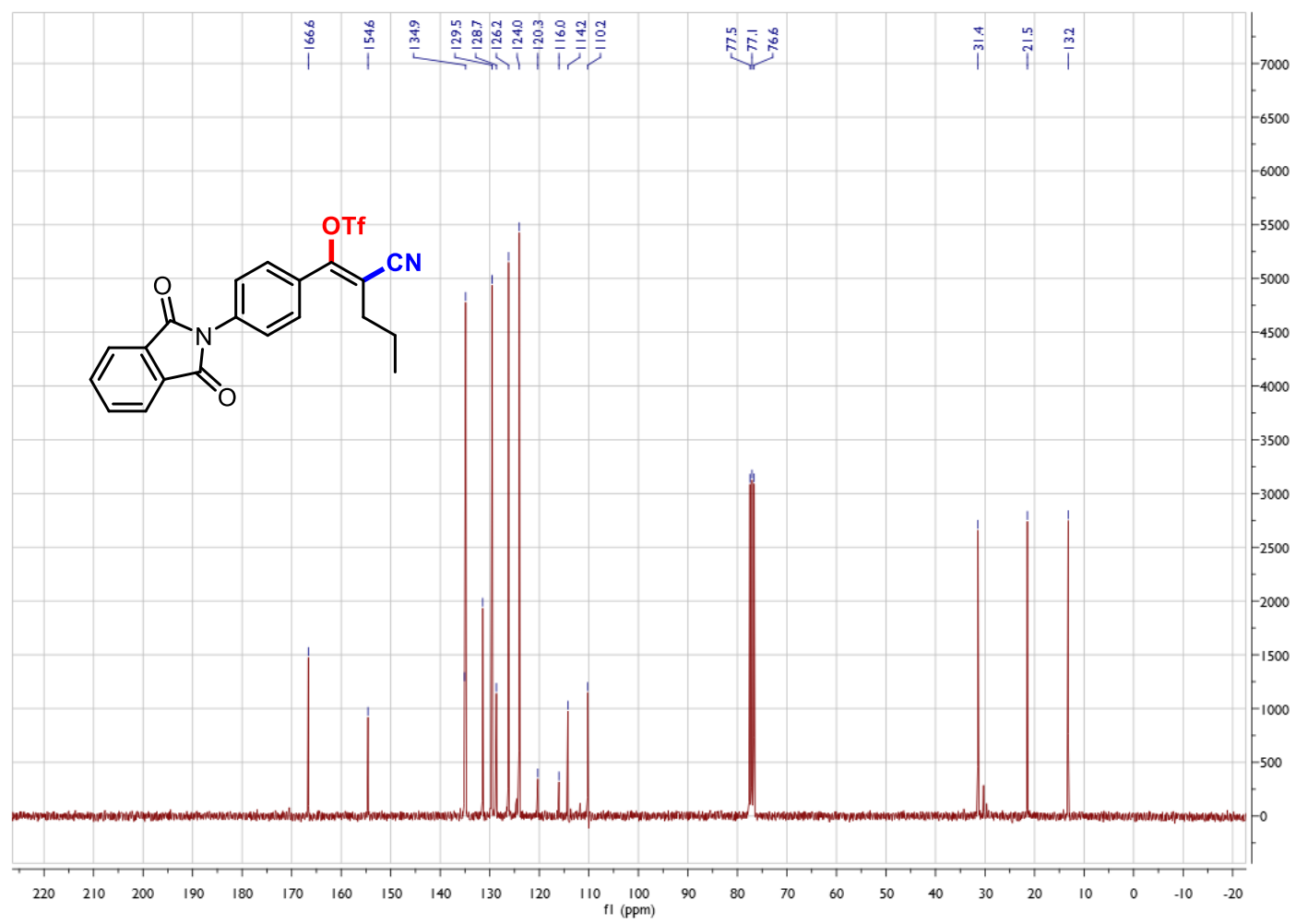


${ }^{19}$ F NMR Spectrum of (Z)-2-cyano-1-(4-(1,3-dioxoisoindolin-2-yl)phenyl)pent-1en-1-yl trifluoromethanesulfonate $2 h$

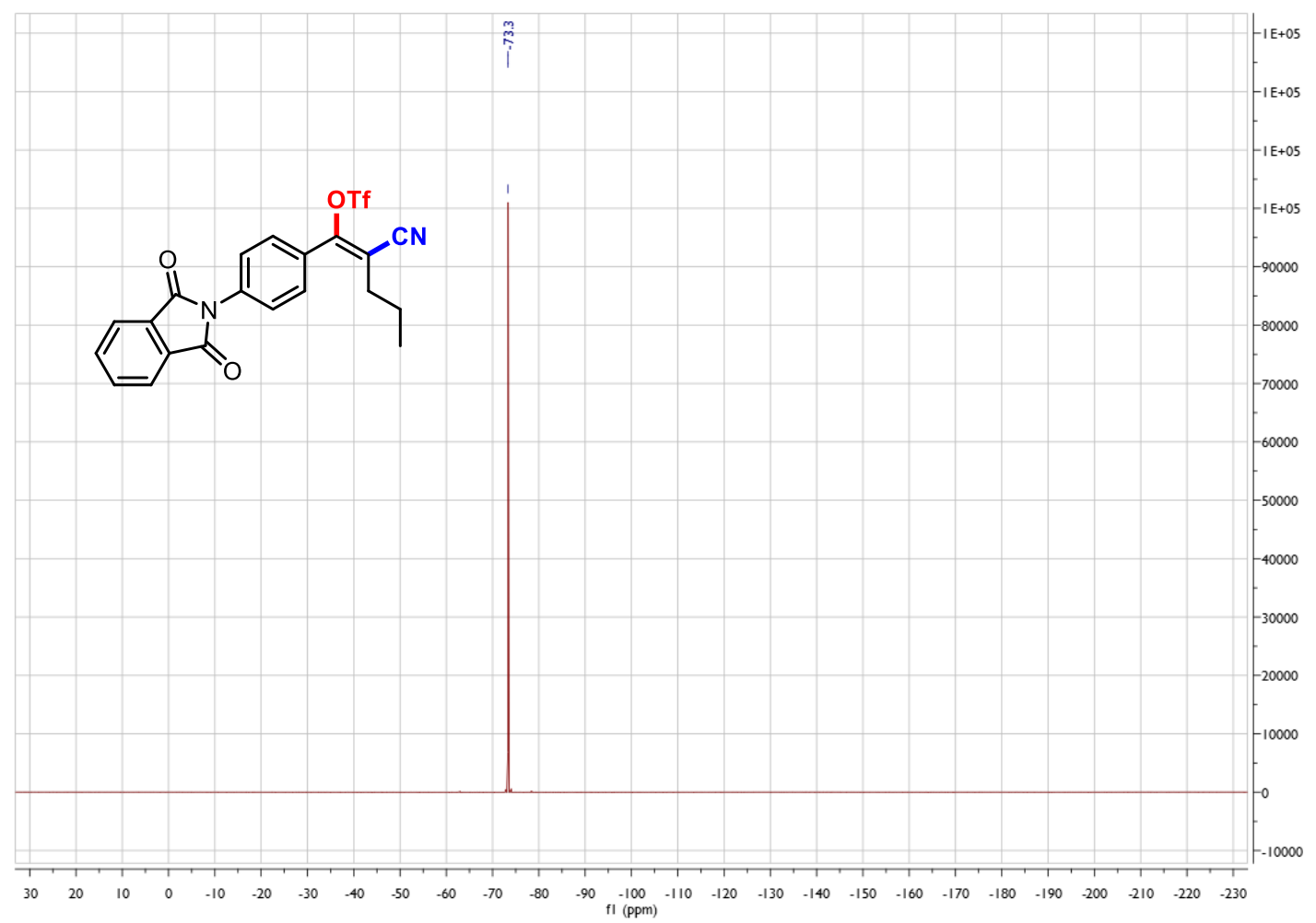


${ }^{1}$ H NMR Spectrum of (Z)-1-(4-Benzoylphenyl)-2-cyanopent-1-en-1-yl trifluoroethanesulfonate $2 i$

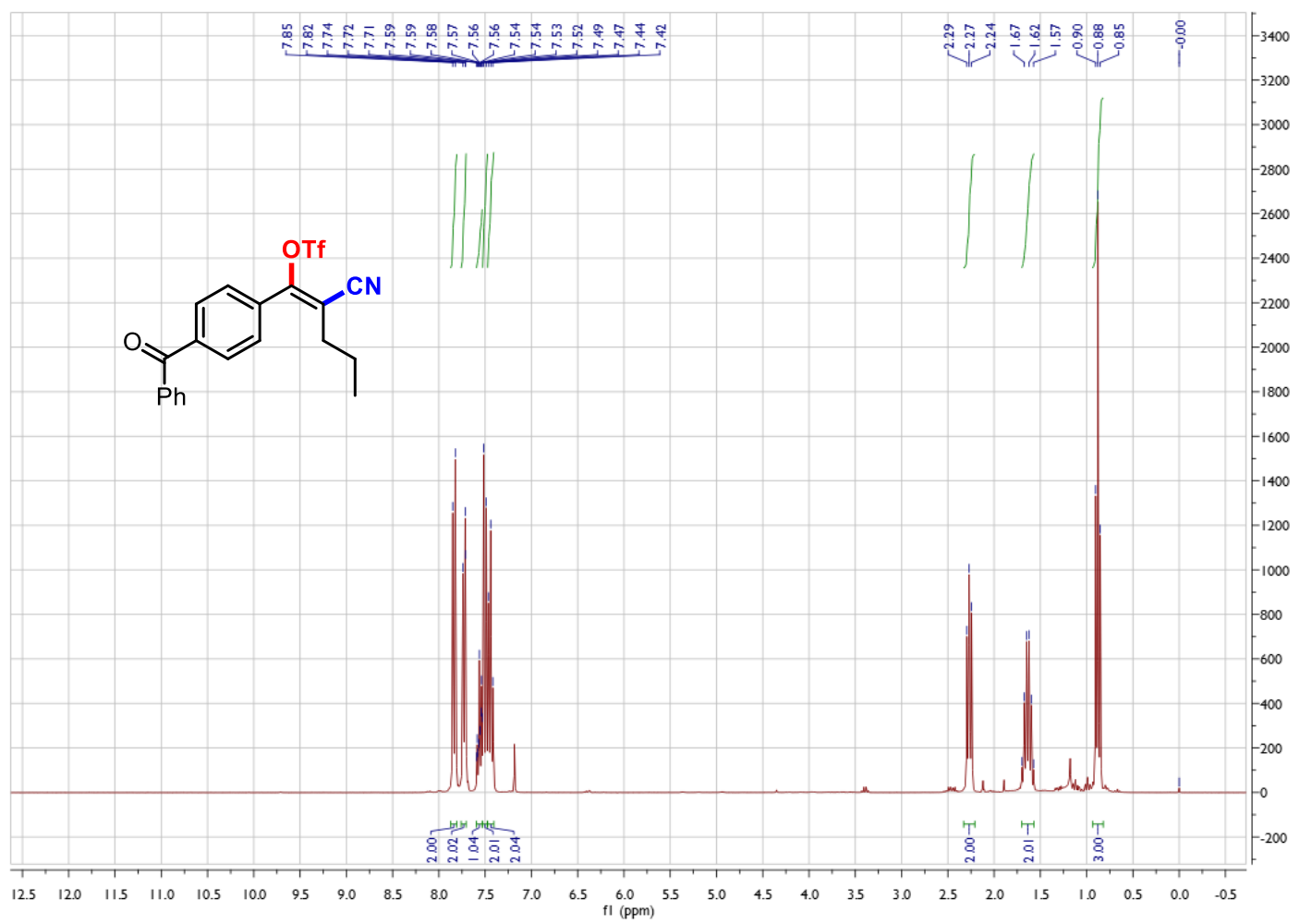

${ }^{13}$ C NMR Spectrum of (Z)-1-(4-Benzoylphenyl)-2-cyanopent-1-en-1-yl trifluoroethanesulfonate $2 i$

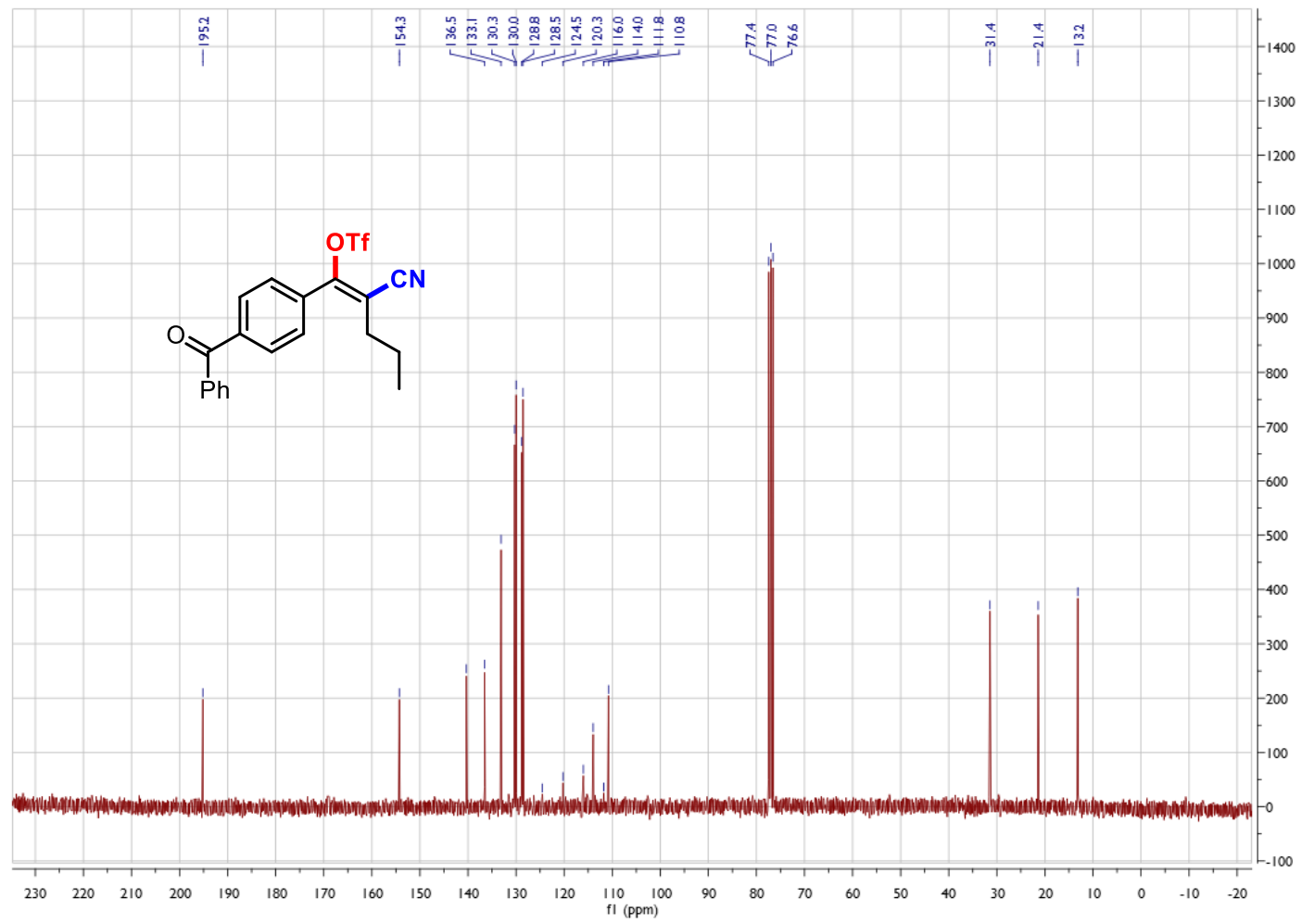


${ }^{19}$ F NMR Spectrum of (Z)-1-(4-Benzoylphenyl)-2-cyanopent-1-en-1-yl trifluoroethanesulfonate $2 \mathrm{i}$

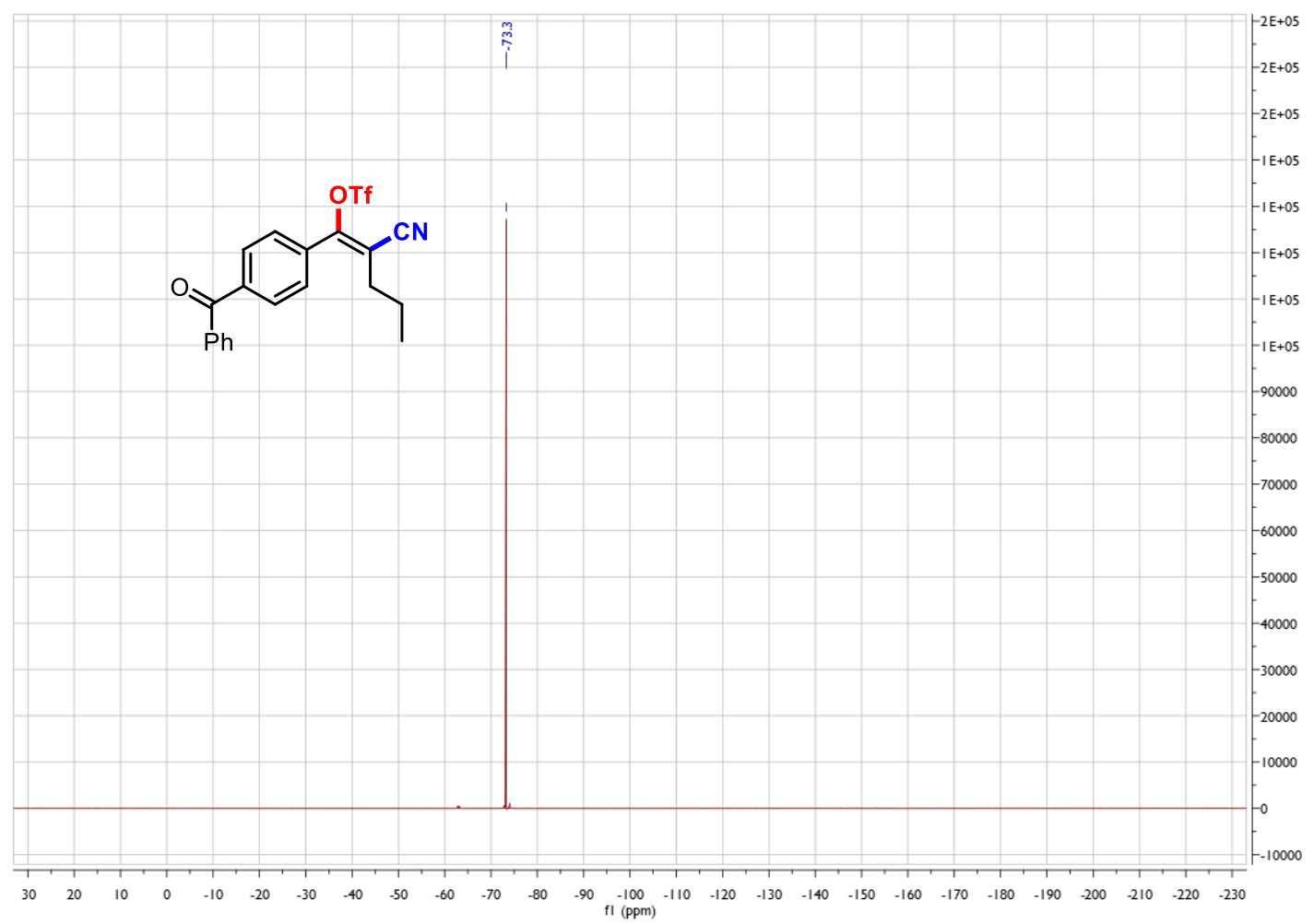


${ }^{1}$ H NMR Spectrum of (Z)-2-cyano-1-(4-(trifluoromethoxy)phenyl)pent-1-en-1-yl trifluoromethanesulfonate $2 \mathbf{j}$

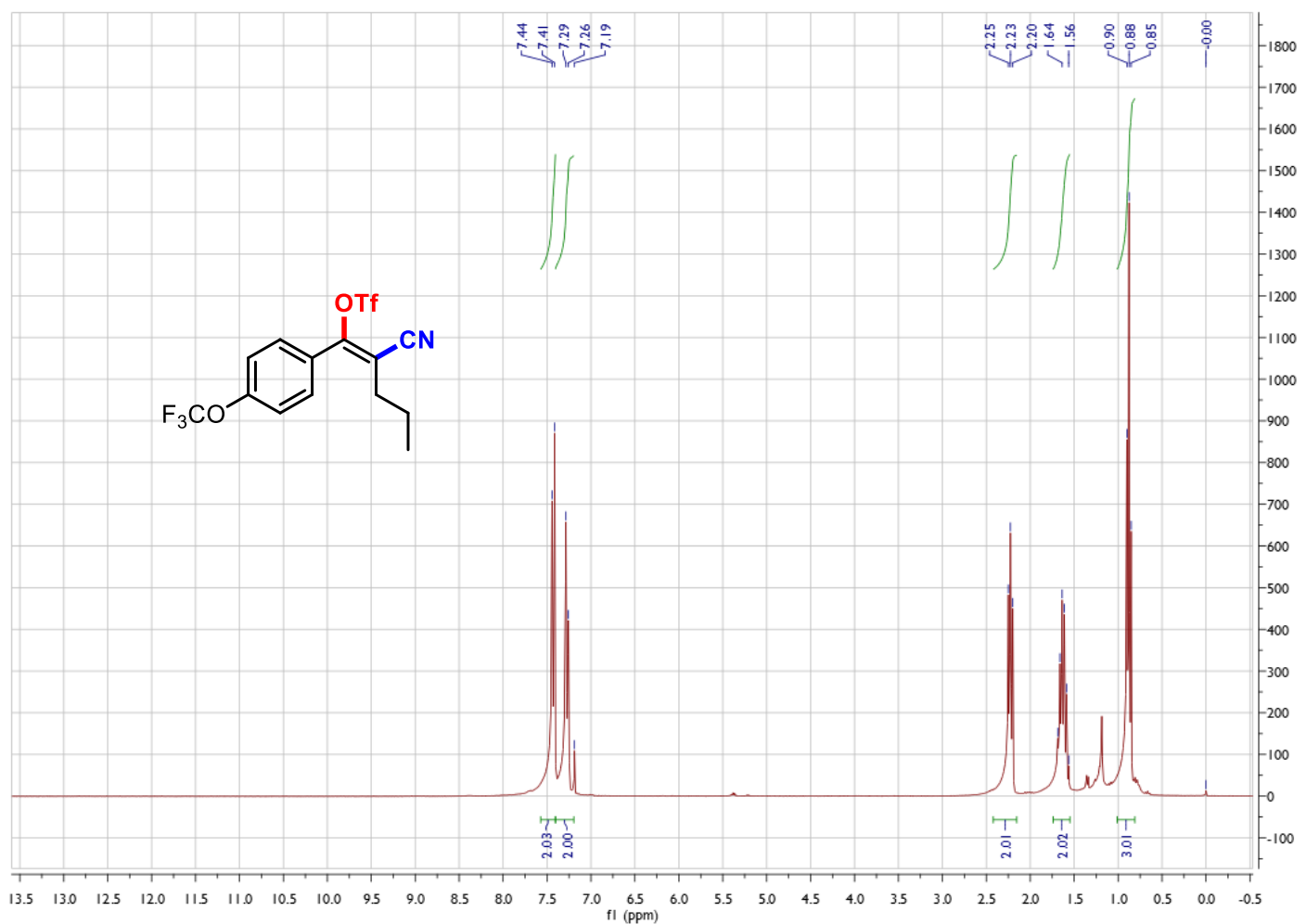

${ }^{13}$ C NMR Spectrum of (Z)-2-cyano-1-(4-(trifluoromethoxy)phenyl)pent-1-en-1-yl trifluoromethanesulfonate $2 \mathbf{j}$

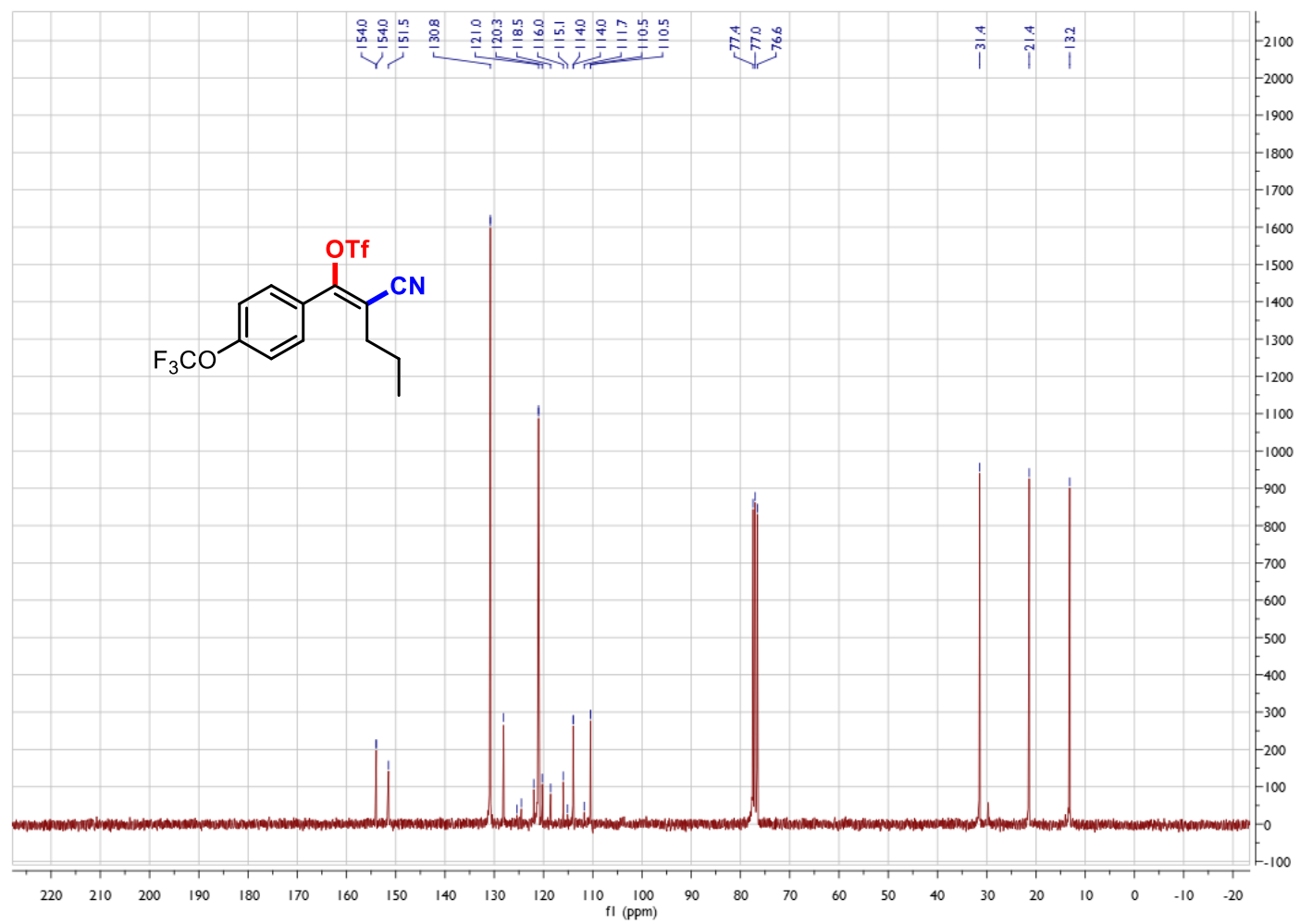


${ }^{19}$ F NMR Spectrum of (Z)-2-cyano-1-(4-(trifluoromethoxy)phenyl)pent-1-en-1-yl trifluoromethanesulfonate $\mathbf{2 j}$

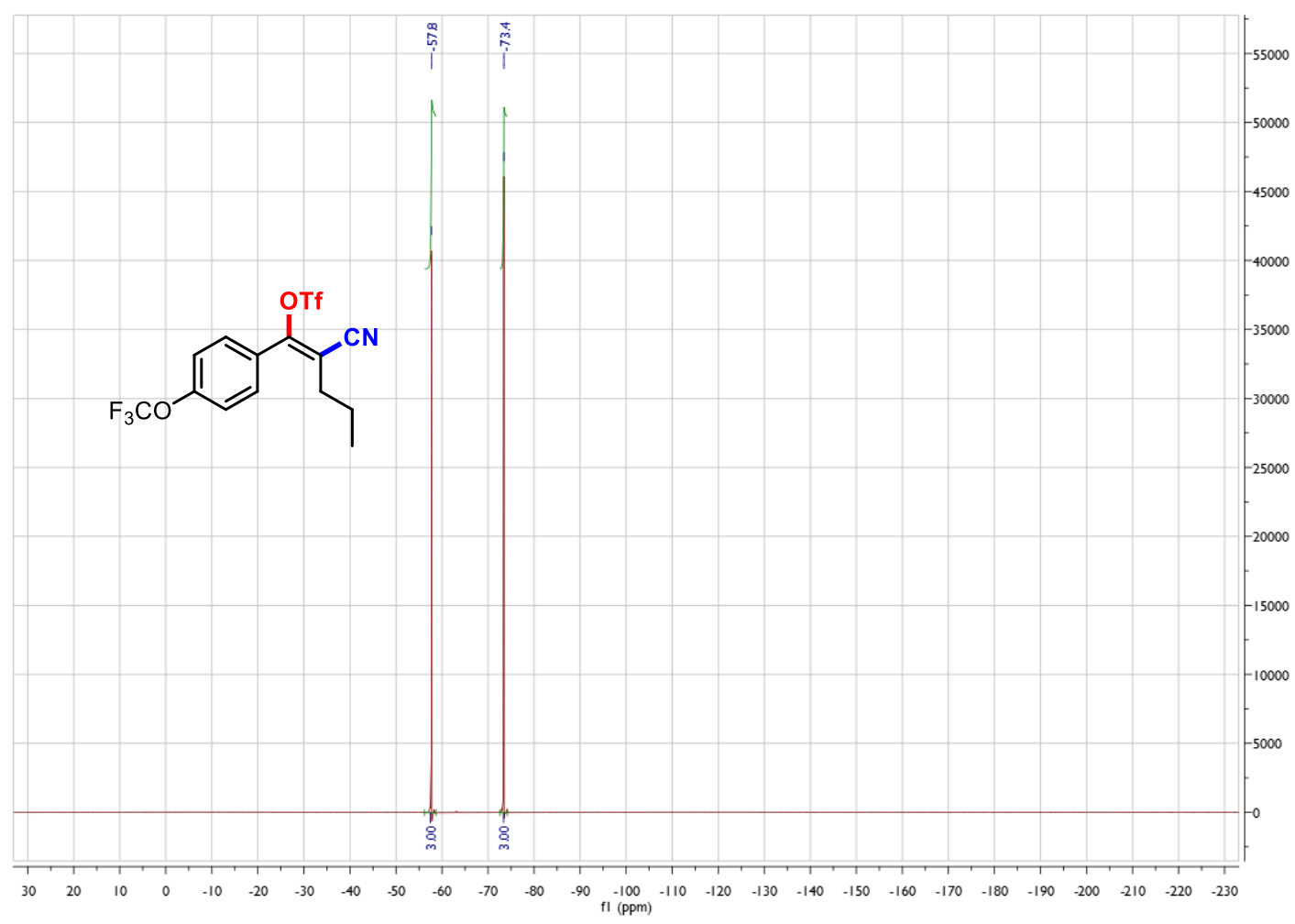


${ }^{1}$ H NMR Spectrum of $(Z)$-2-cyano-1-(4-((trifluoromethyl)thio)phenyl)pent-1-en-1yl trifluoromethanesulfonate $2 k$

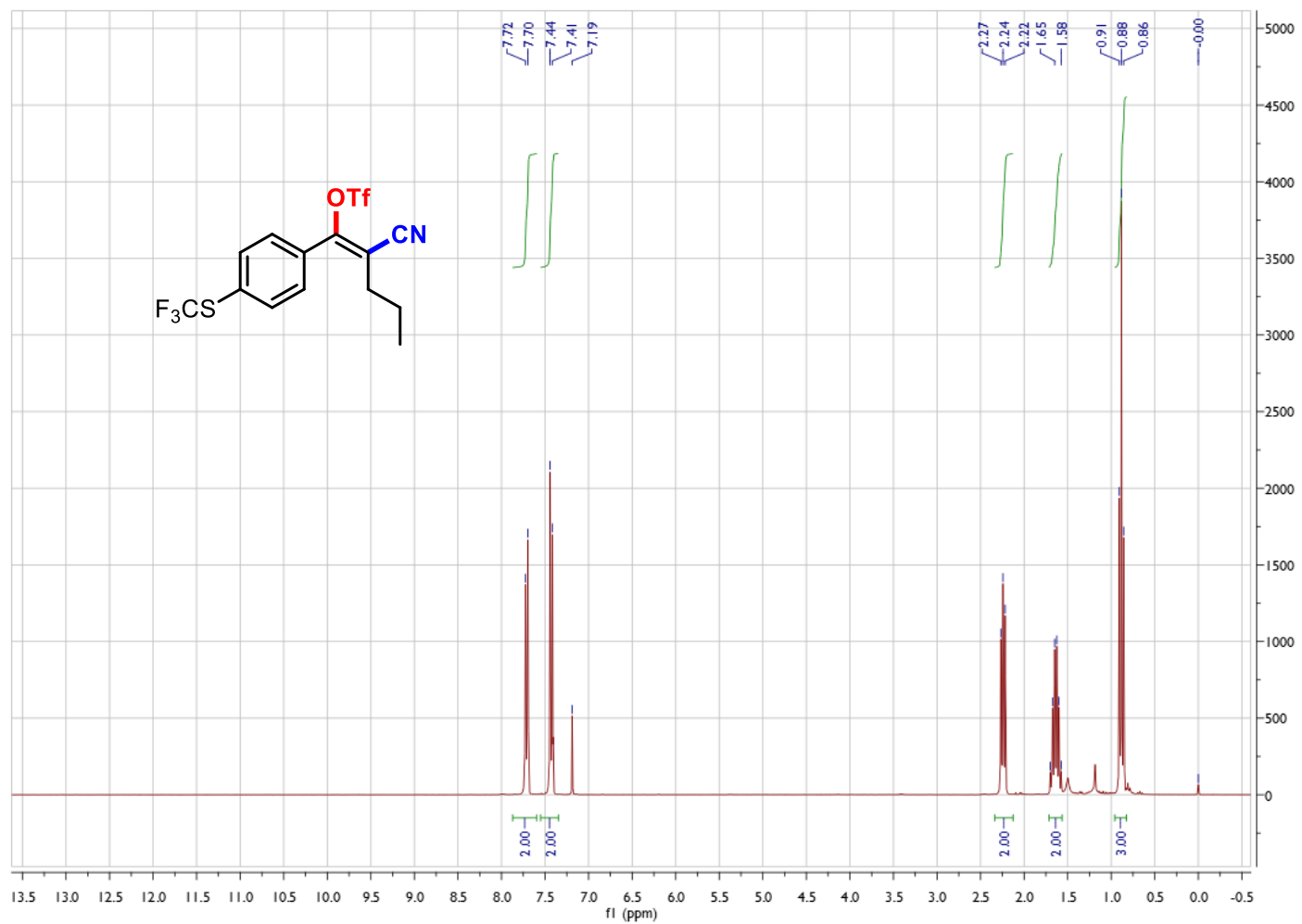

${ }^{13}$ C NMR Spectrum of (Z)-2-cyano-1-(4-((trifluoromethyl)thio)phenyl)pent-1-en1-yl trifluoromethanesulfonate $2 \mathrm{k}$

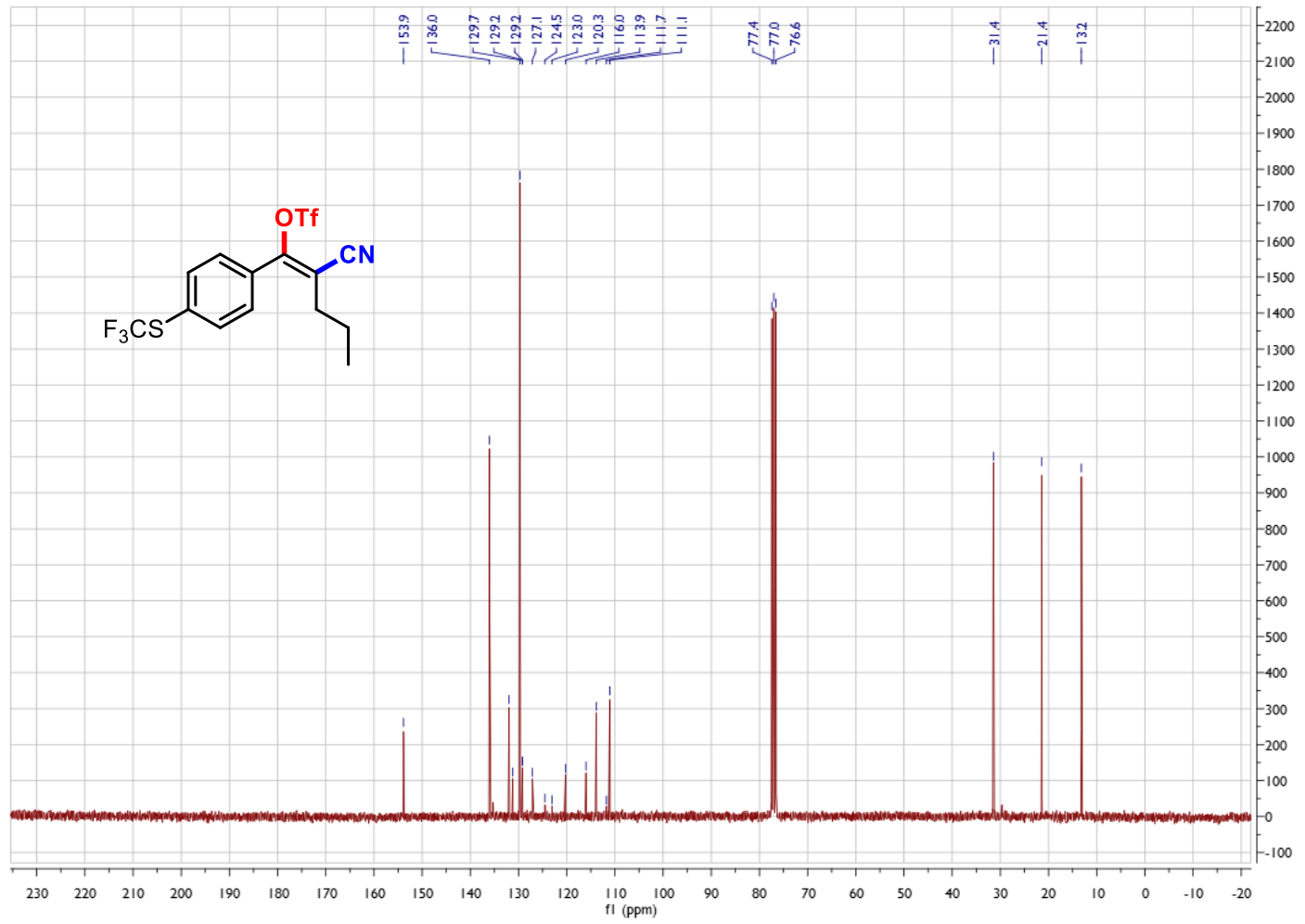


${ }^{19}$ F NMR Spectrum of (Z)-2-cyano-1-(4-((trifluoromethyl)thio)phenyl)pent-1-en1-yl trifluoromethanesulfonate $2 k$

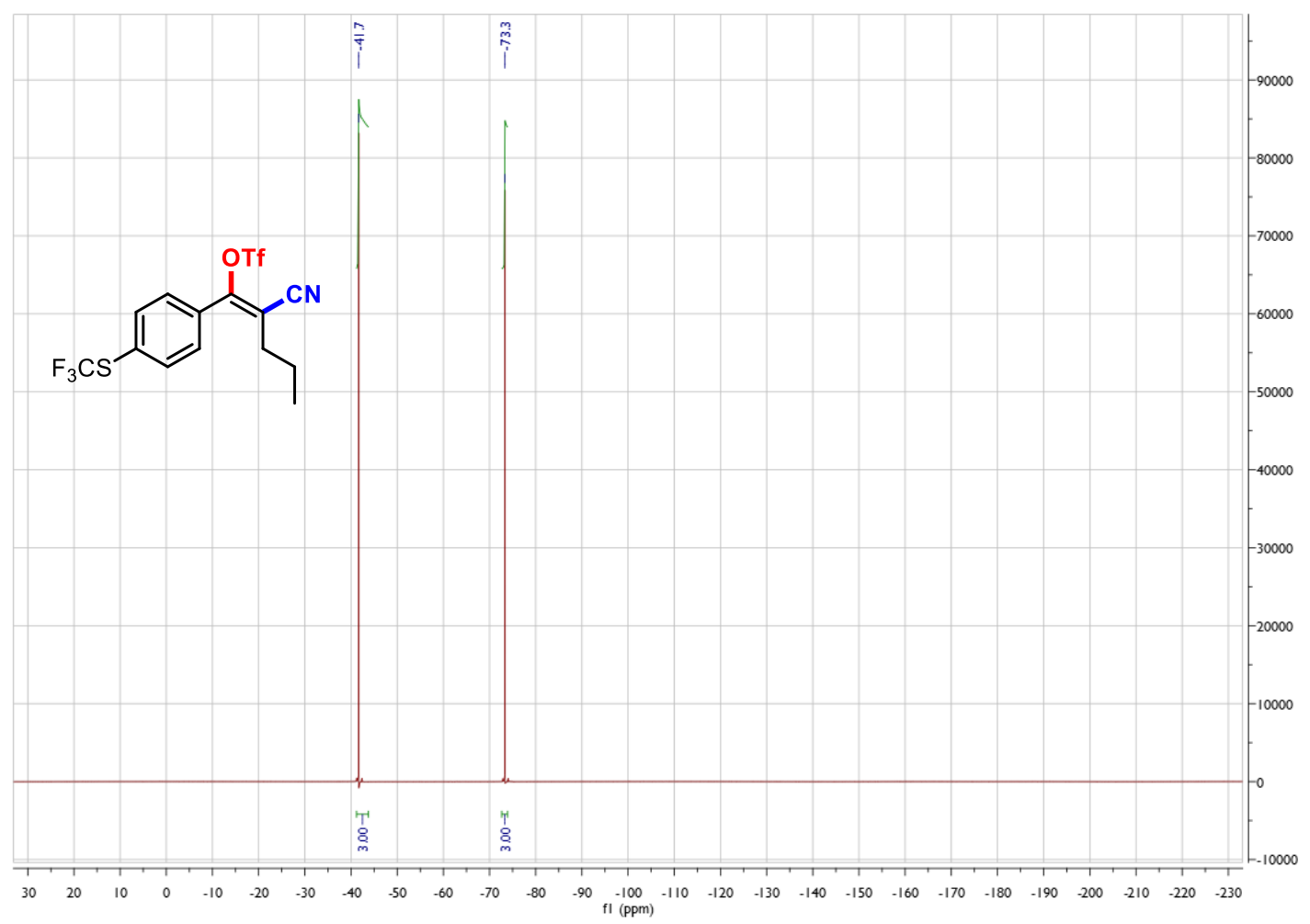


${ }^{1}$ H NMR Spectrum of (Z)-2-cyano-1-(4-((trifluoromethyl)thio)phenyl)pent-1-en-1yl trifluoromethanesulfonate 21

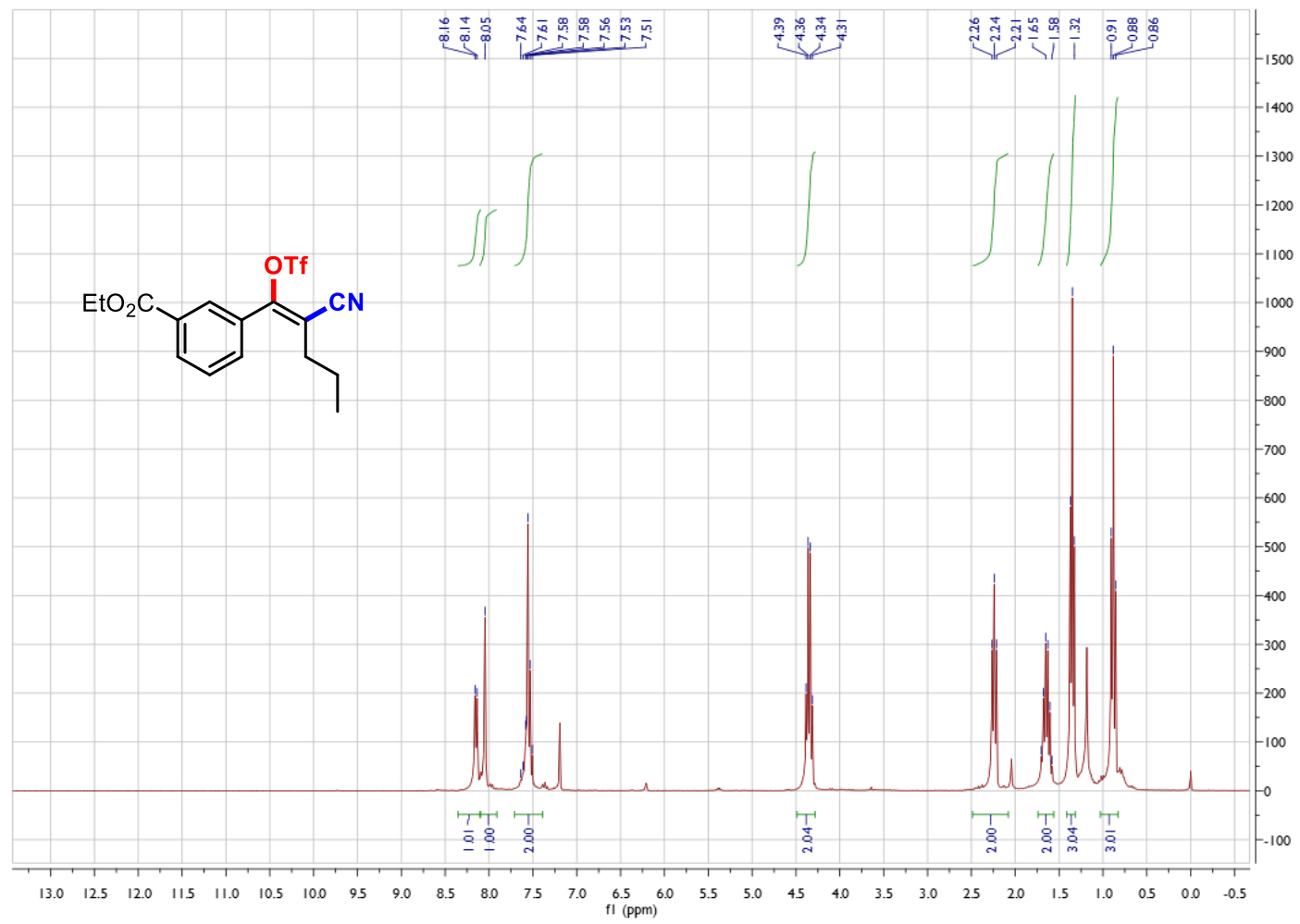

${ }^{13}$ C NMR Spectrum of (Z)-2-cyano-1-(4-((trifluoromethyl)thio)phenyl)pent-1-en1-yl trifluoromethanesulfonate $2 \mathrm{I}$

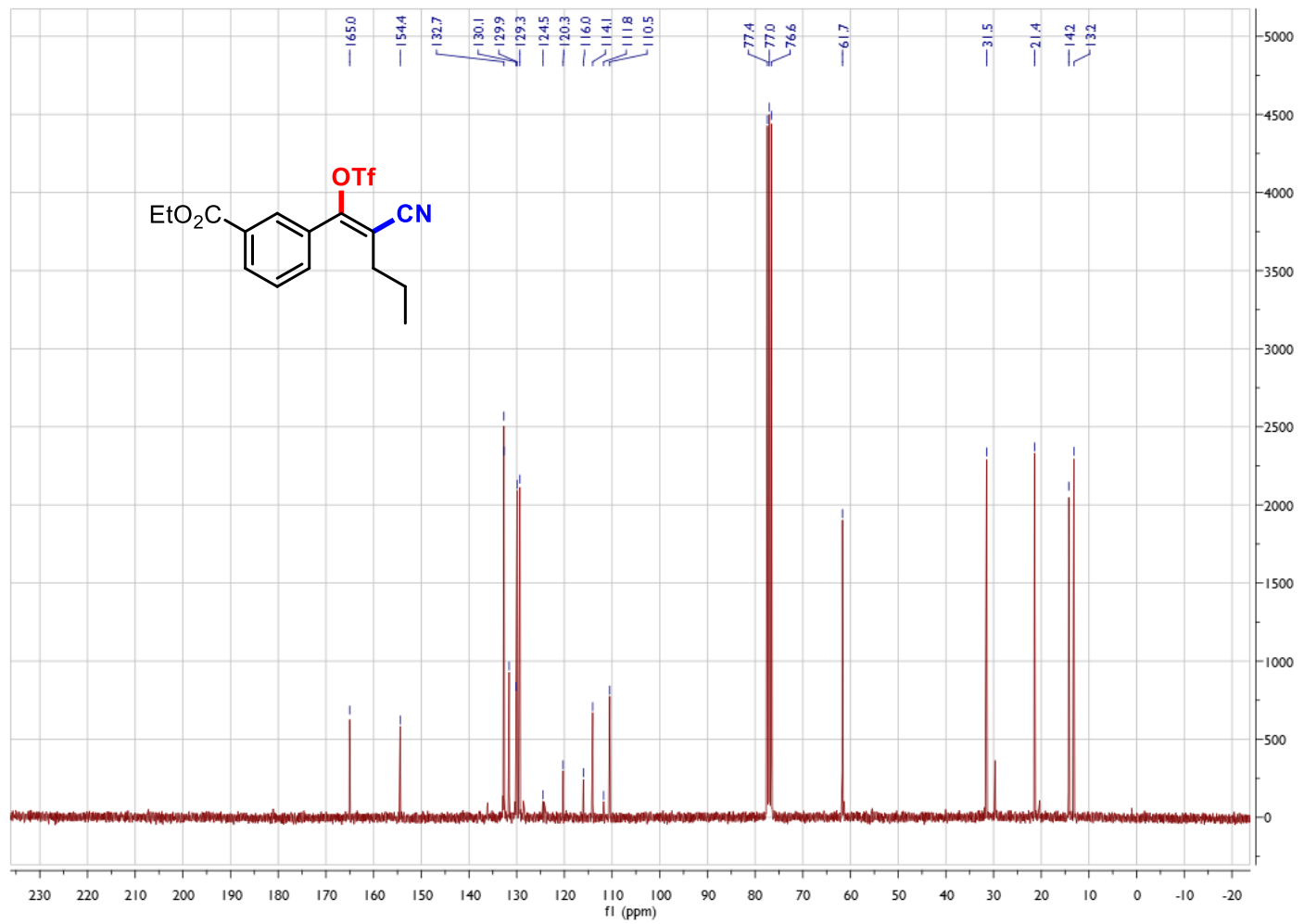


${ }^{19}$ F NMR Spectrum of (Z)-2-cyano-1-(4-((trifluoromethyl)thio)phenyl)pent-1-en1-yl trifluoromethanesulfonate 21

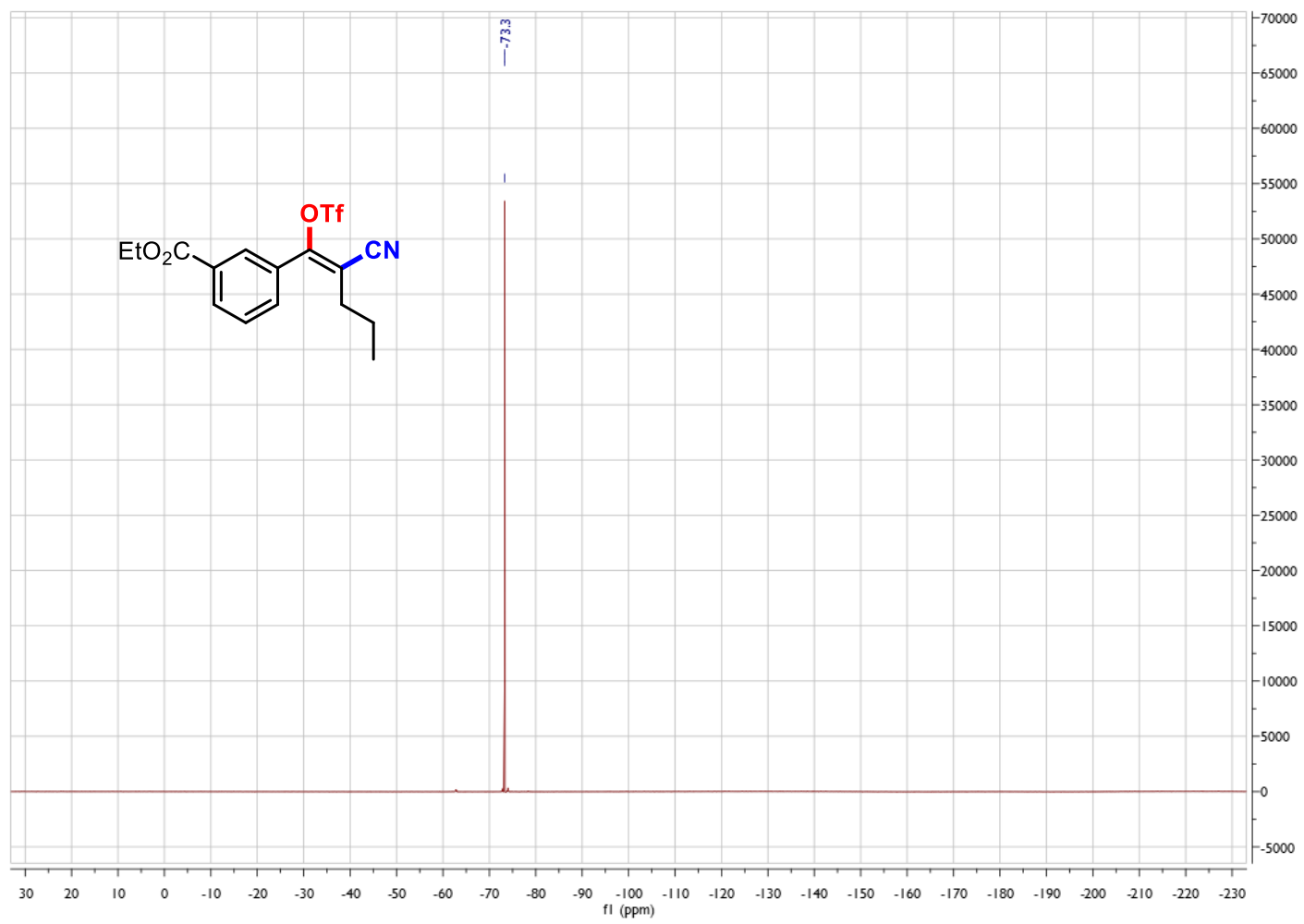


${ }^{1} \mathrm{H}$ NMR Spectrum of (Z)-2-cyano-1-(3-formylphenyl)pent-1-en-1-yl trifluoromethanesulfonate $2 \mathrm{~m}$

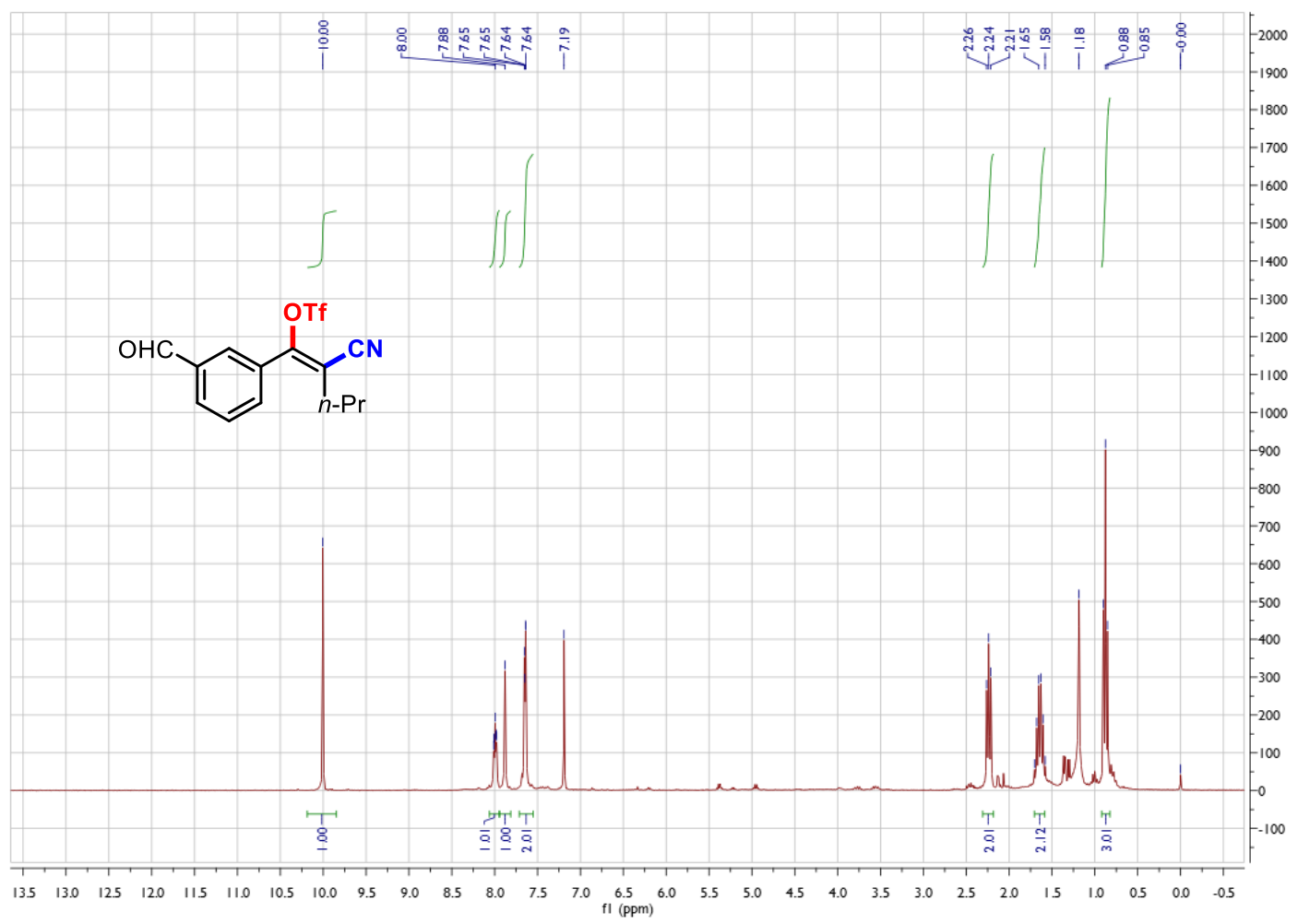

${ }^{13}$ C NMR Spectrum of (Z)-2-cyano-1-(3-formylphenyl)pent-1-en-1-yl trifluoromethanesulfonate $2 \mathrm{~m}$

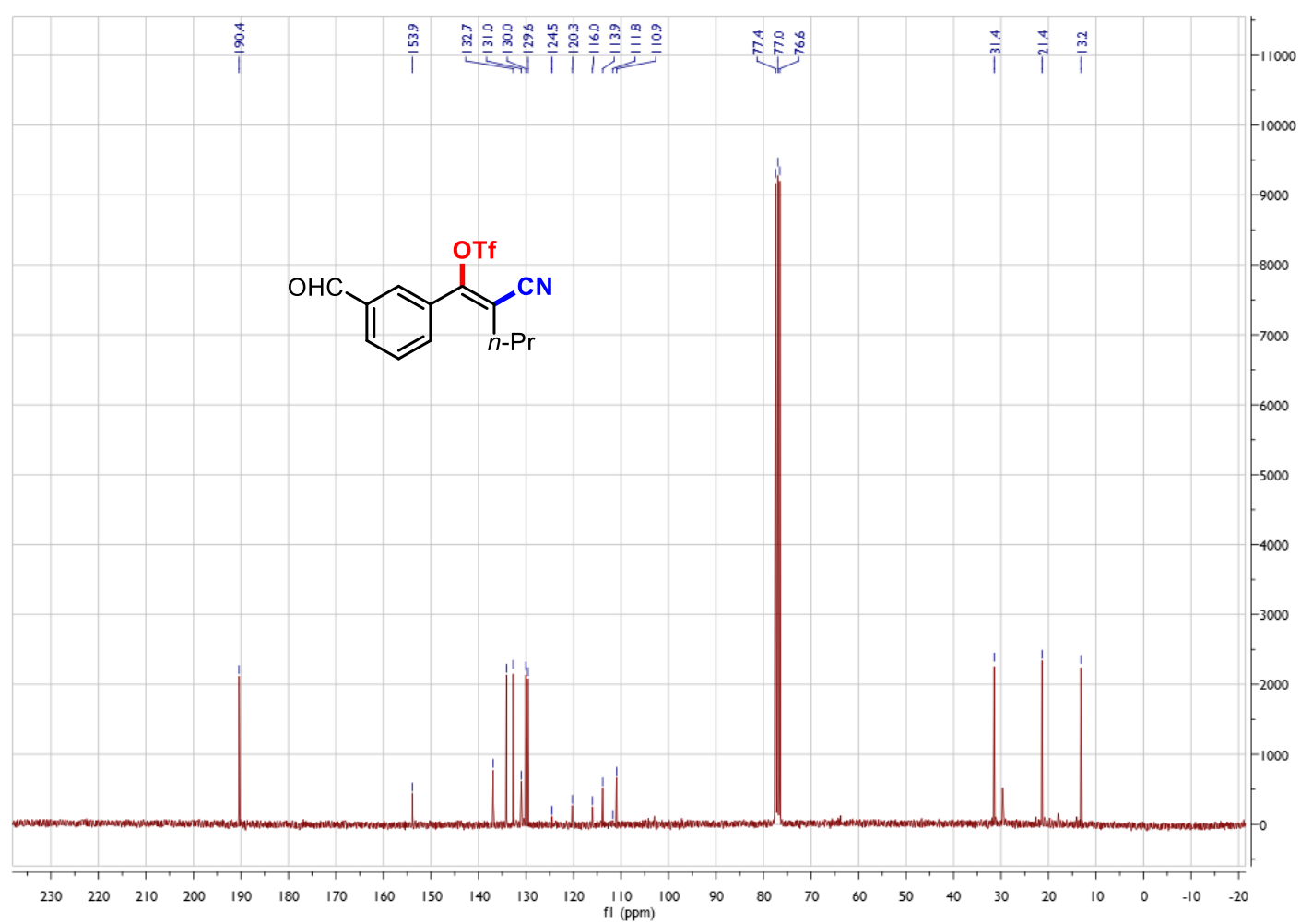


${ }^{19}$ F NMR Spectrum of (Z)-2-cyano-1-(3-formylphenyl)pent-1-en-1-yl trifluoroethanesulfonate $2 \mathrm{~m}$

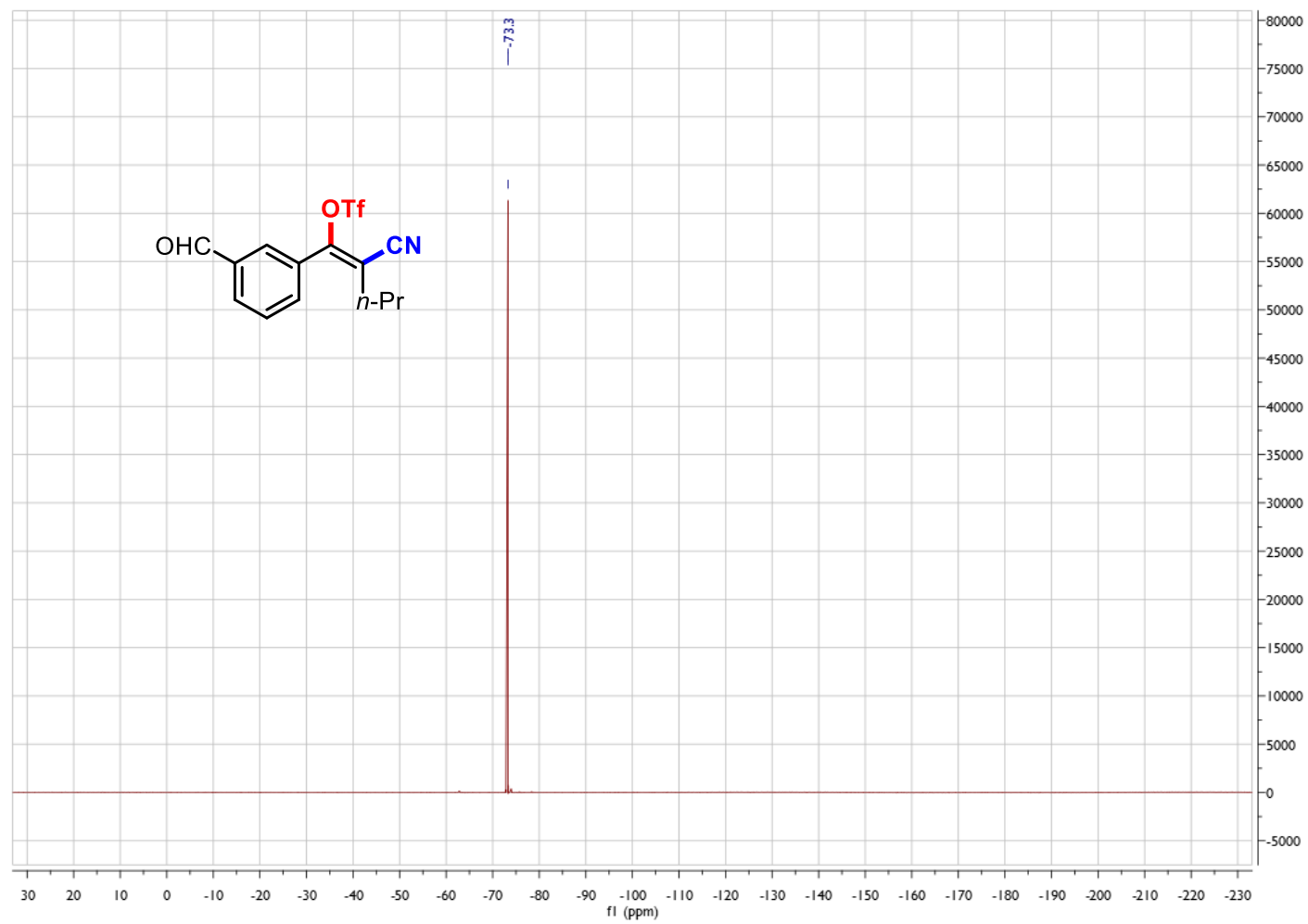


${ }^{1}$ H NMR Spectrum of (Z)-2-cyano-1-(o-tolyl)pent-1-en-1-yl trifluoromethanesulfonate 2n

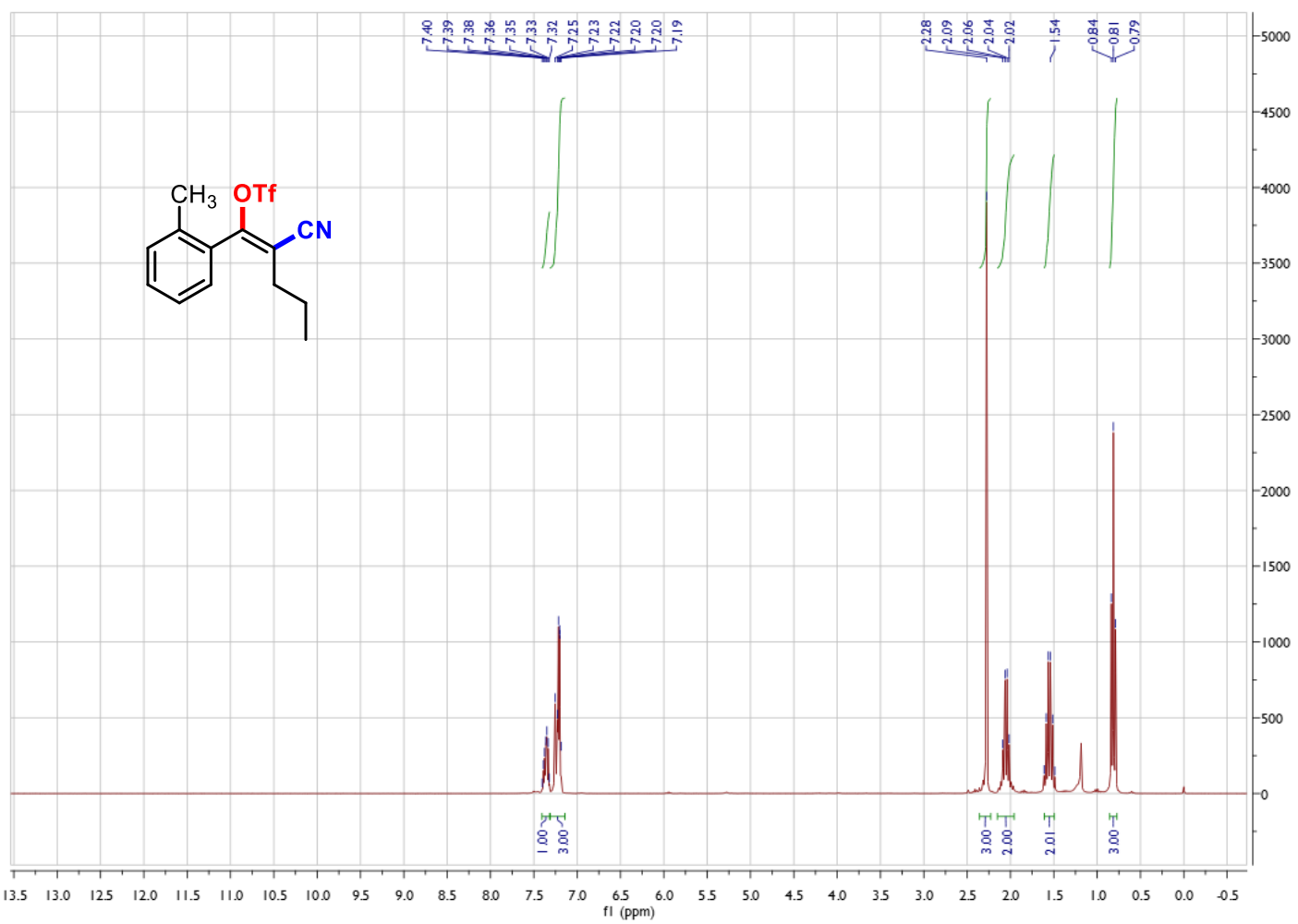

${ }^{13} \mathrm{C}$ NMR Spectrum of (Z)-2-cyano-1-(o-tolyl)pent-1-en-1-yl trifluoromethanesulfonate 2 n

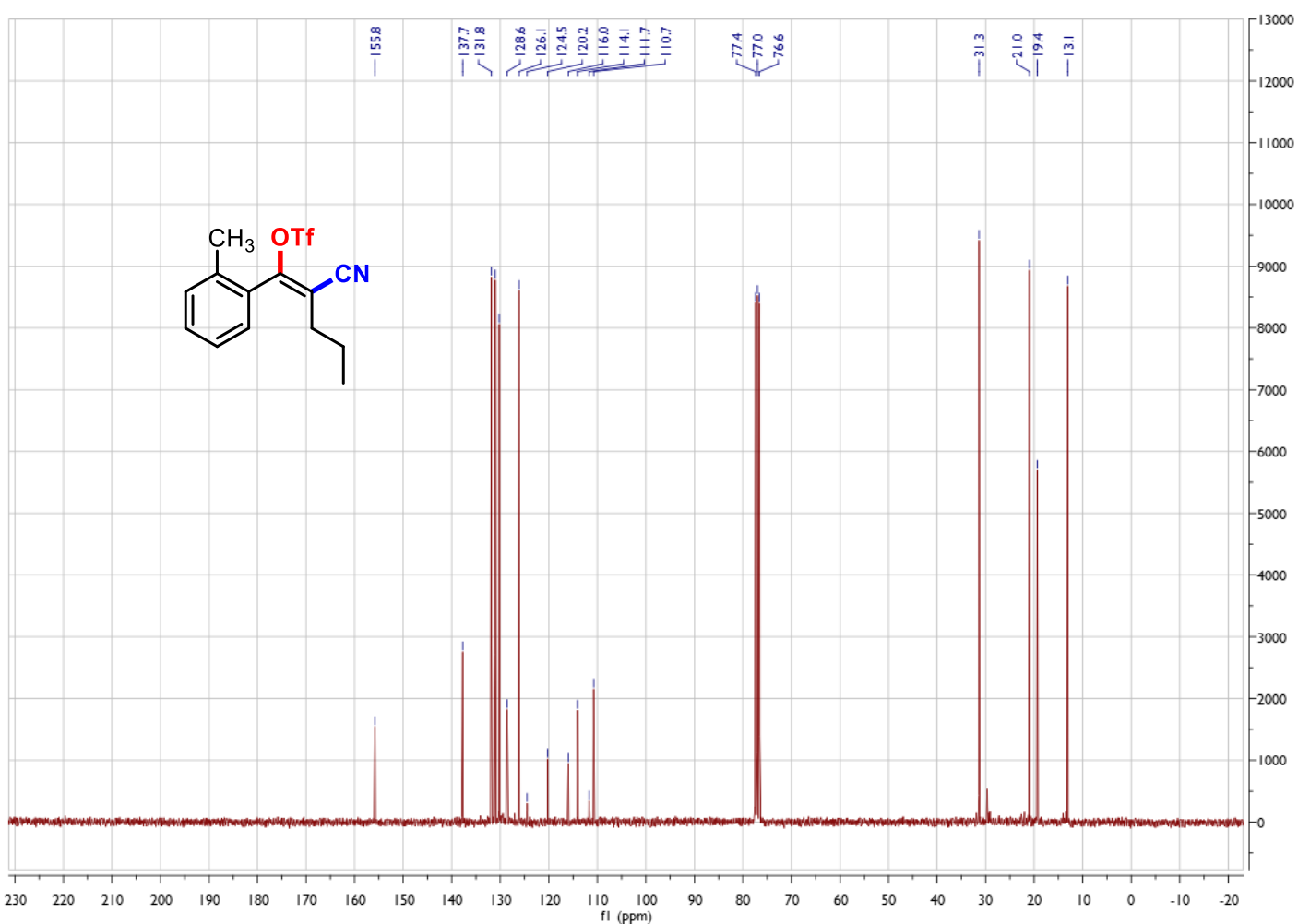


${ }^{19}$ F NMR Spectrum of (Z)-2-cyano-1-(o-tolyl)pent-1-en-1-yl trifluoromethanesulfonate $2 n$

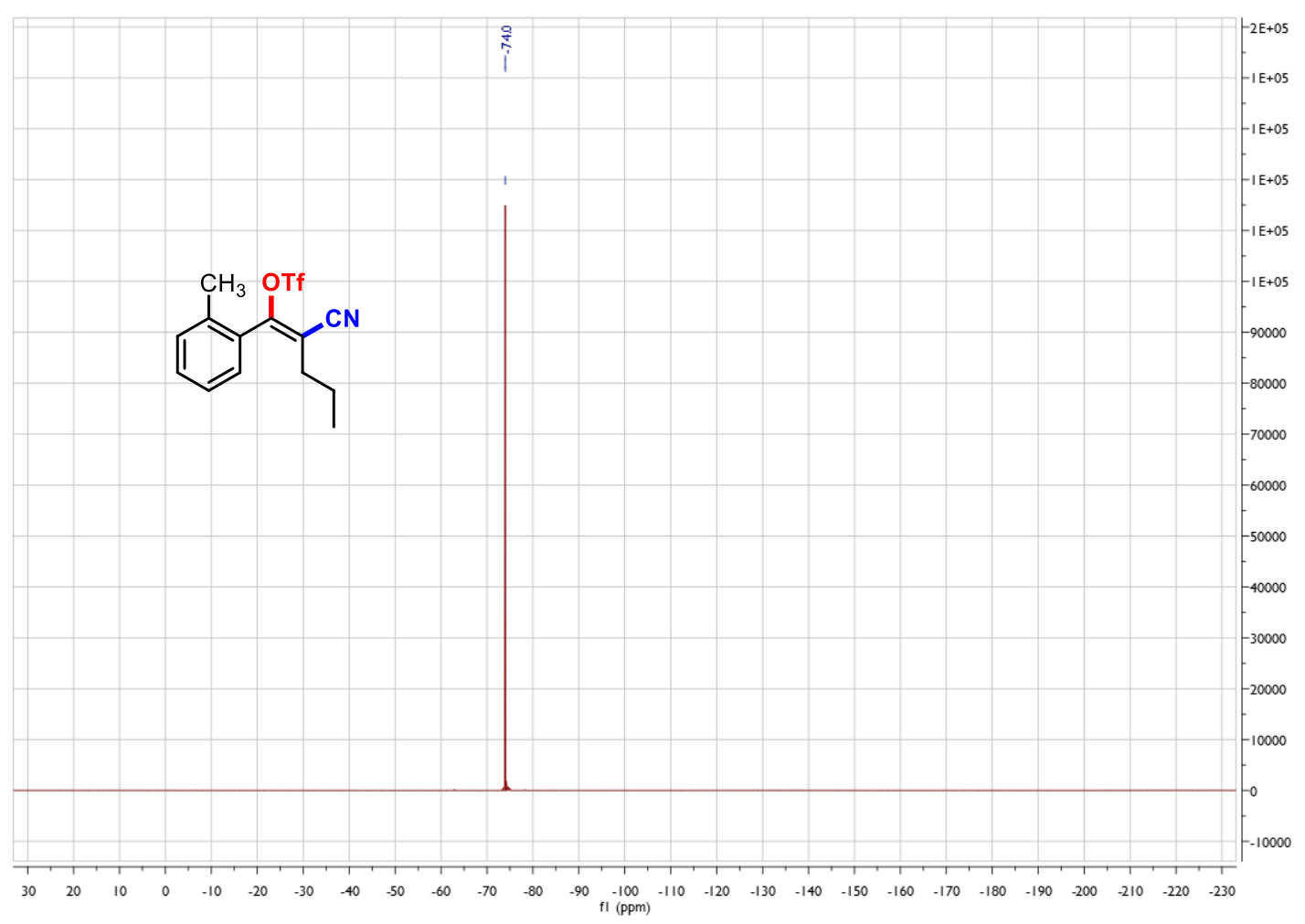


${ }^{1} \mathrm{H}$ NMR Spectrum of (Z)-1-(4-bromophenyl)-4-cyanohept-3-en-1-yn-3-yl trifluoromethanesulfonate 20

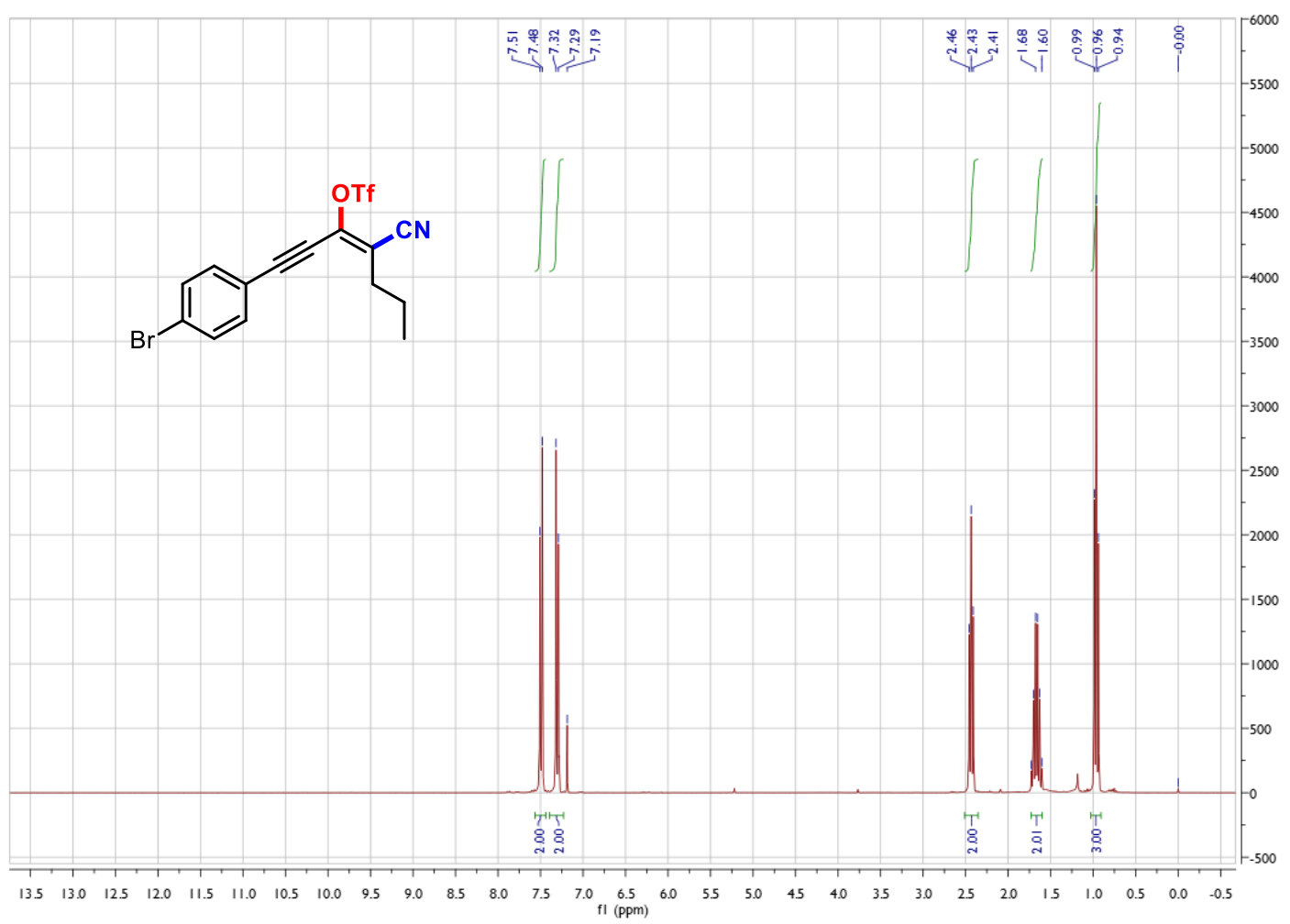

${ }^{13} \mathrm{C}$ NMR Spectrum of (Z)-1-(4-bromophenyl)-4-cyanohept-3-en-1-yn-3-yl trifluoromethanesulfonate 20

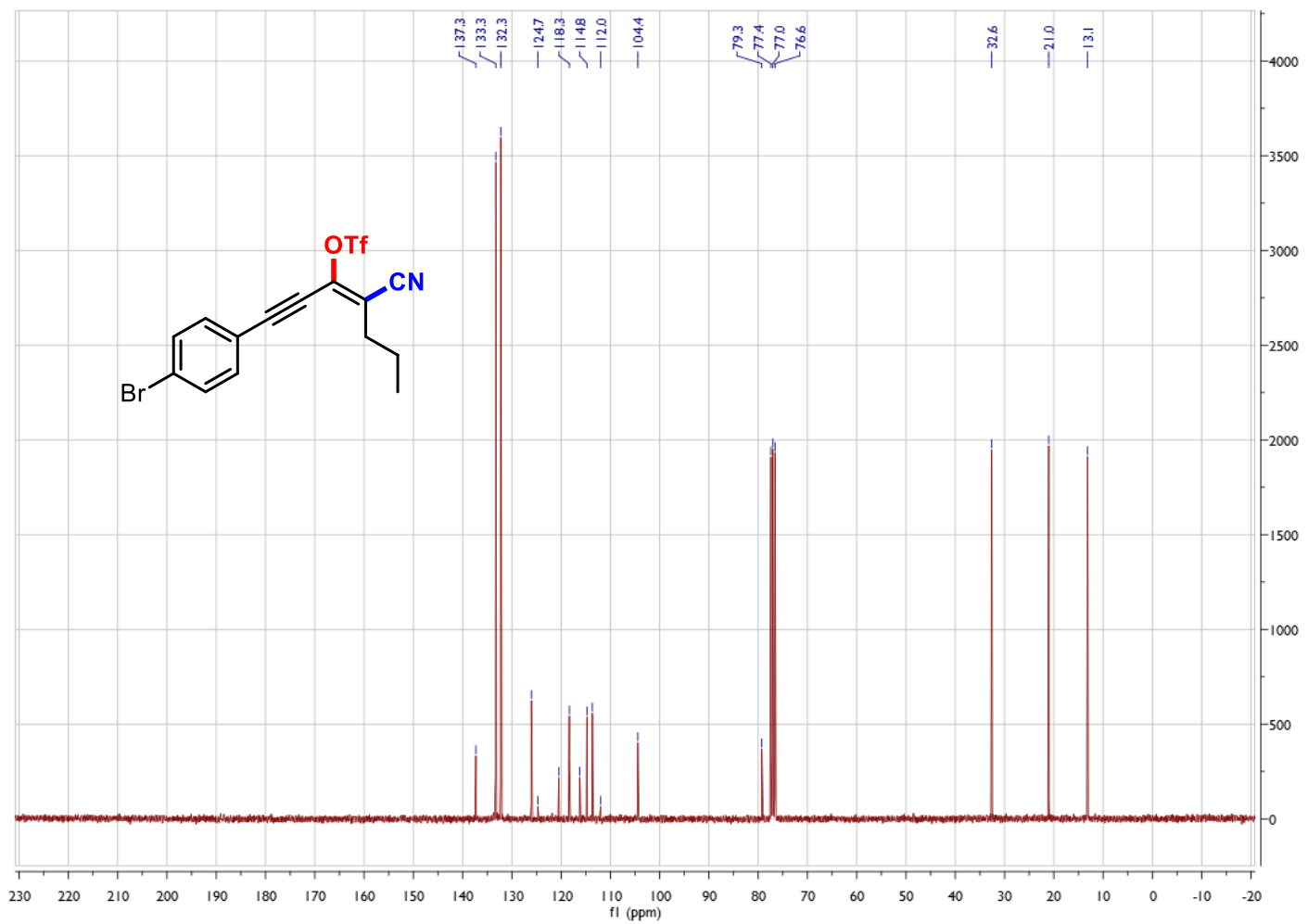


${ }^{19}$ F NMR Spectrum of (Z)-1-(4-bromophenyl)-4-cyanohept-3-en-1-yn-3-yl trifluoromethanesulfonate 20

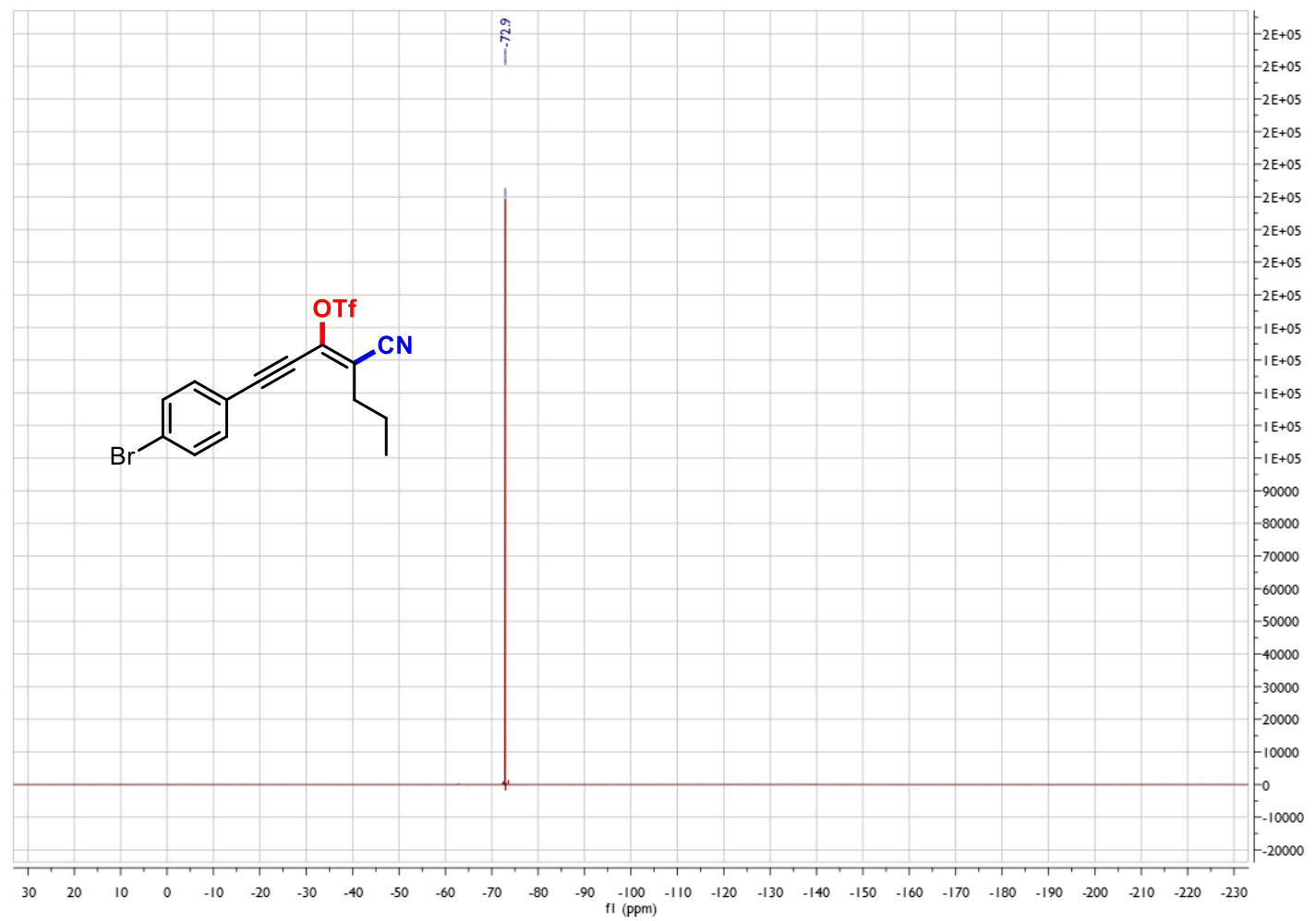

NOESY1D Spectrum of (Z)-1-(4-bromophenyl)-4-cyanohept-3-en-1-yn-3-yl trifluoromethanesulfonate 20

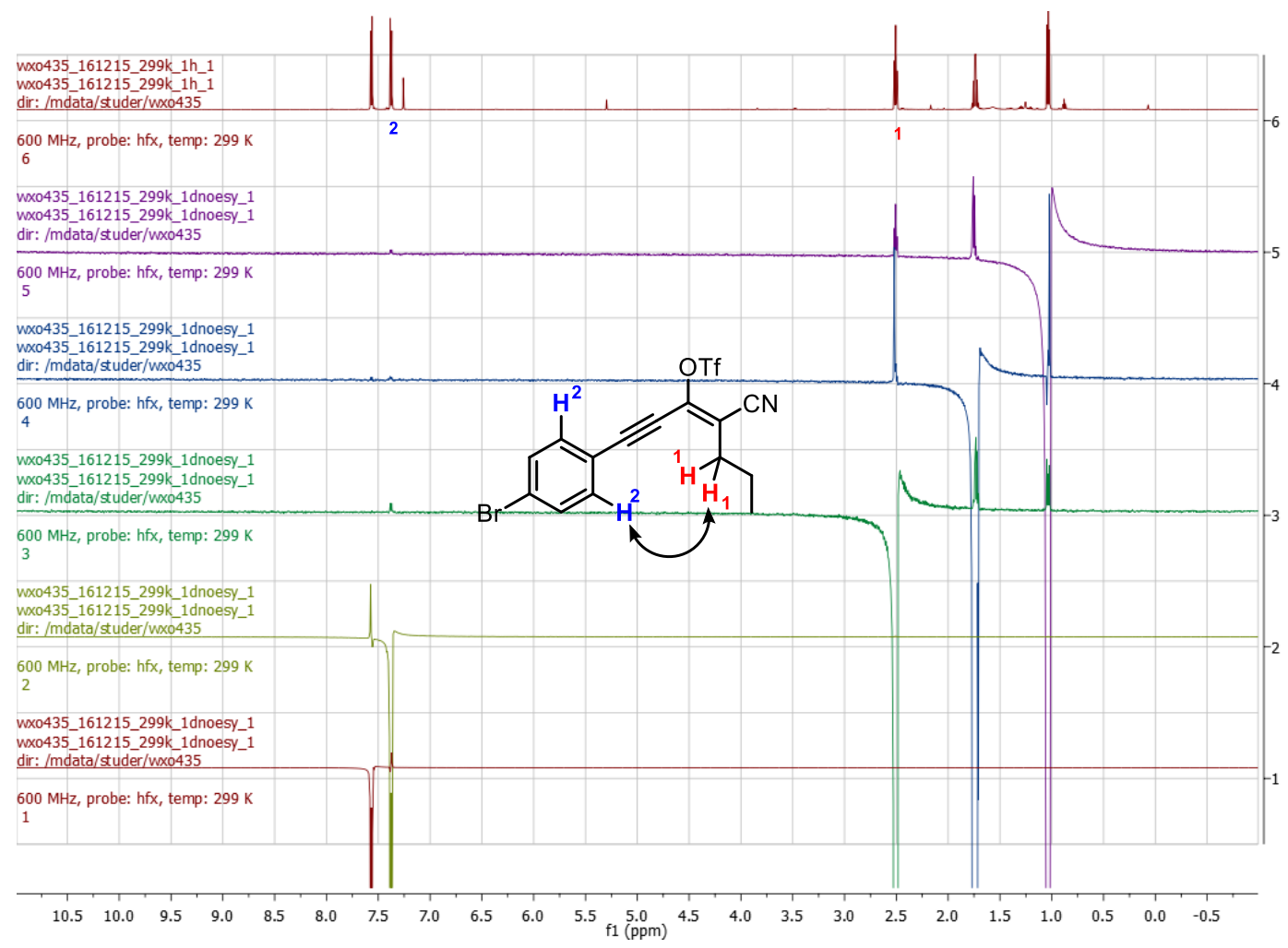


${ }^{13}$ C/1 H GHMBCAD Spectrum of (Z)-1-(4-bromophenyl)-4-cyanohept-3-en-1-yn3-yl trifluoromethanesulfonate 20

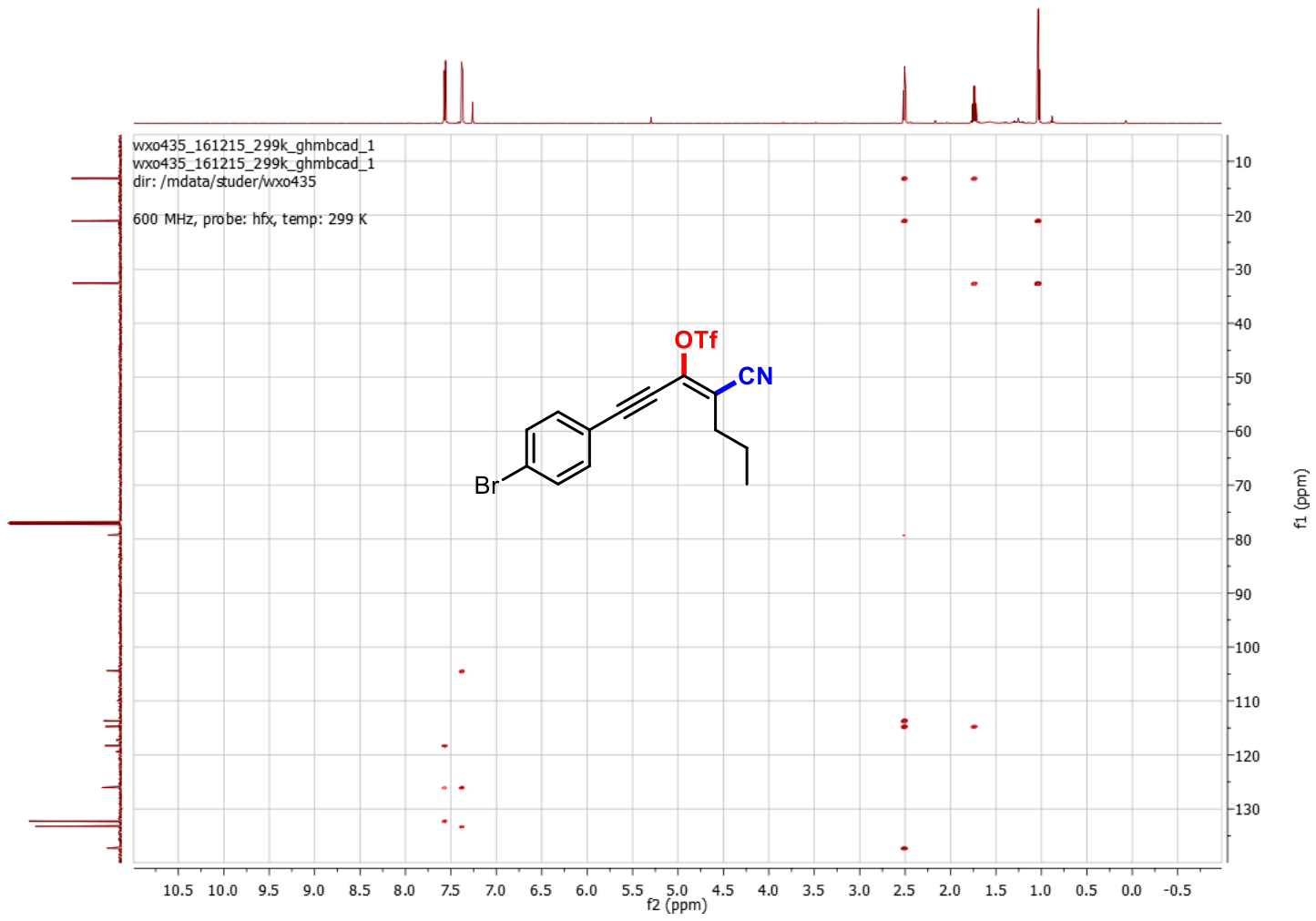


${ }^{1} \mathrm{H}$ NMR Spectrum of $(1 E, 3 Z)-1-(4-c h l o r o p h e n y l)-4-c y a n o h e p t a-1,3-d i e n-3-y l$ trifluoromethanesulfonate $2 p$

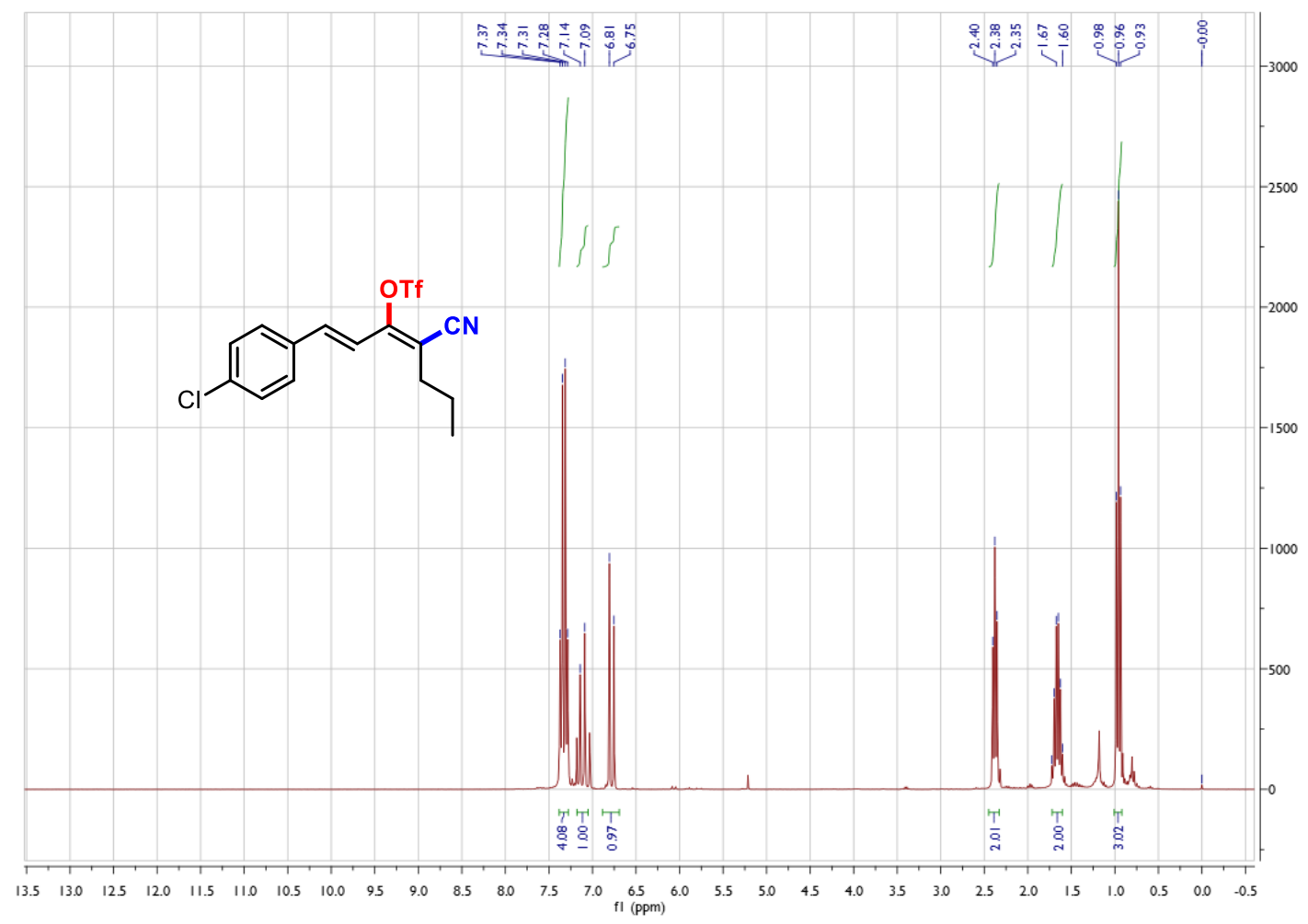

${ }^{13} \mathrm{C}$ NMR Spectrum of $(1 E, 3 Z)-1-(4-c h l o r o p h e n y l)-4-c y a n o h e p t a-1,3-d i e n-3-y l$ trifluoromethanesulfonate $2 p$

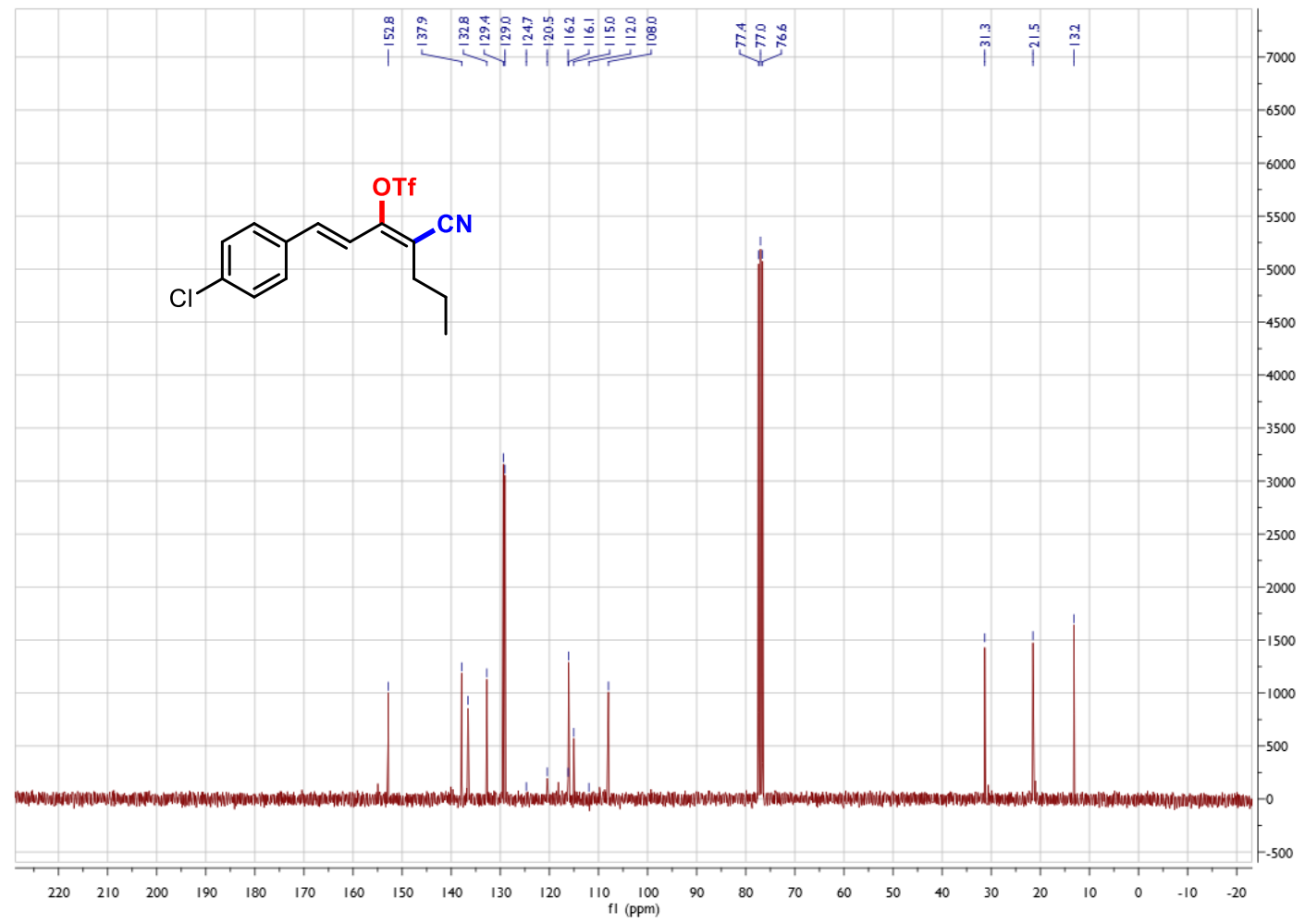


${ }^{19}$ F NMR Spectrum of $(1 E, 3 Z)-1-(4-c h l o r o p h e n y l)-4-c y a n o h e p t a-1,3-d i e n-3-y l$ trifluoromethanesulfonate $2 p$

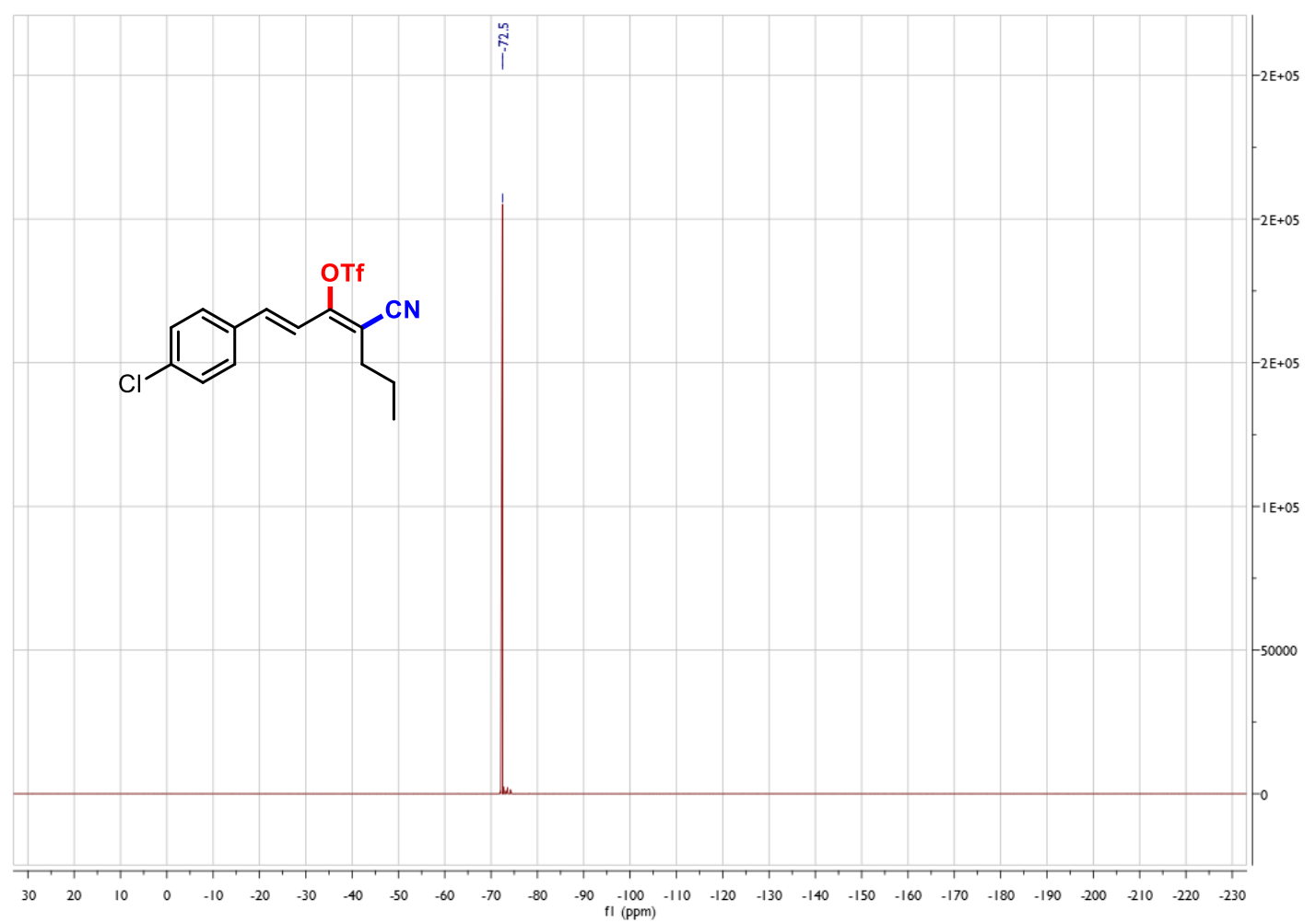

NOESY1D Spectrum of (1E, 3Z)-1-(4-chlorophenyl)-4-cyanohepta-1,3-dien-3-yl trifluoromethanesulfonate $2 \mathbf{p}$

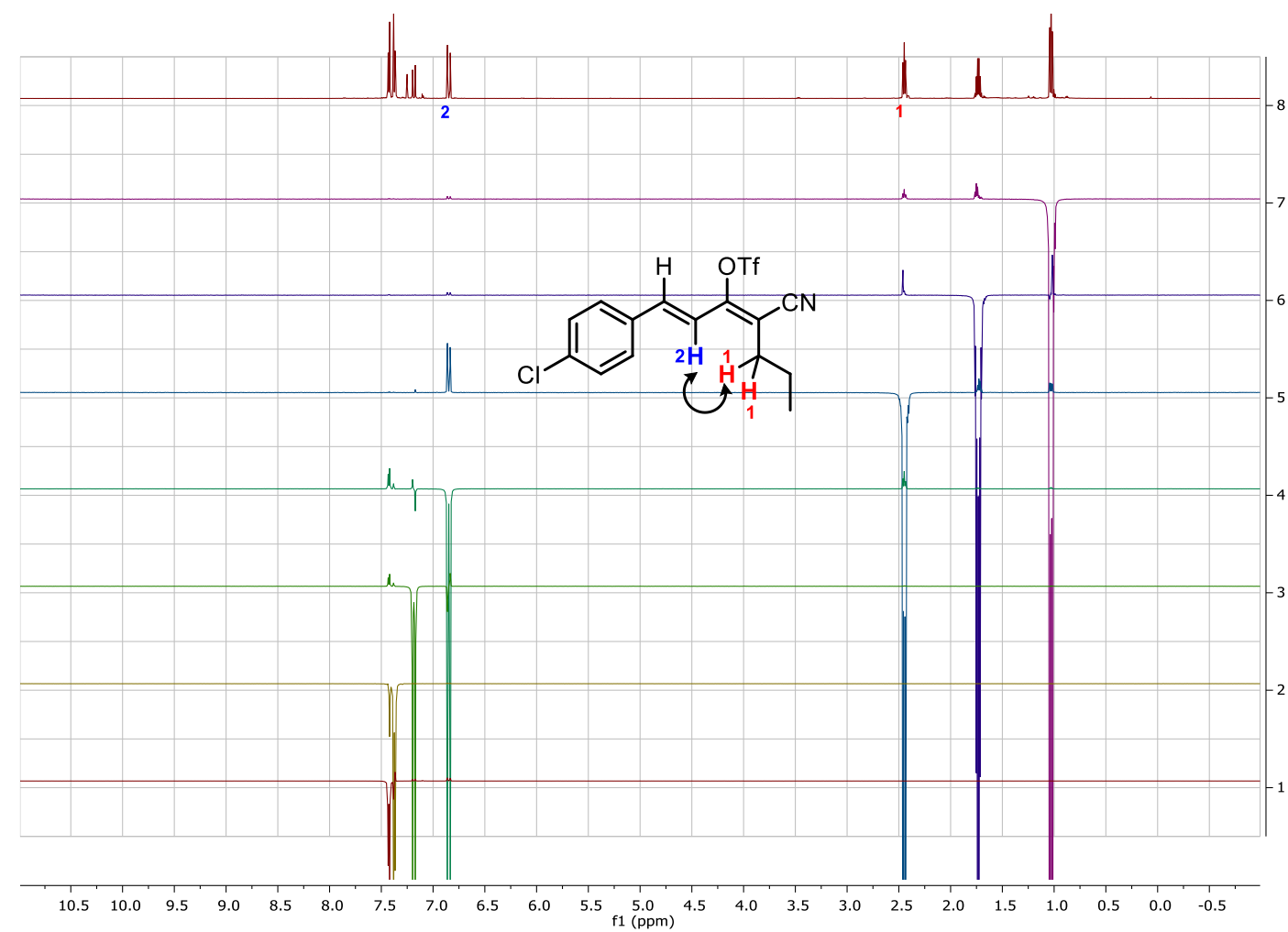


${ }^{1}$ H NMR Spectrum of (Z)-1-(4-bromophenyl)-5-chloro-2-cyanopent-1-en-1-yl trifluoromethanesulfonate $2 q$

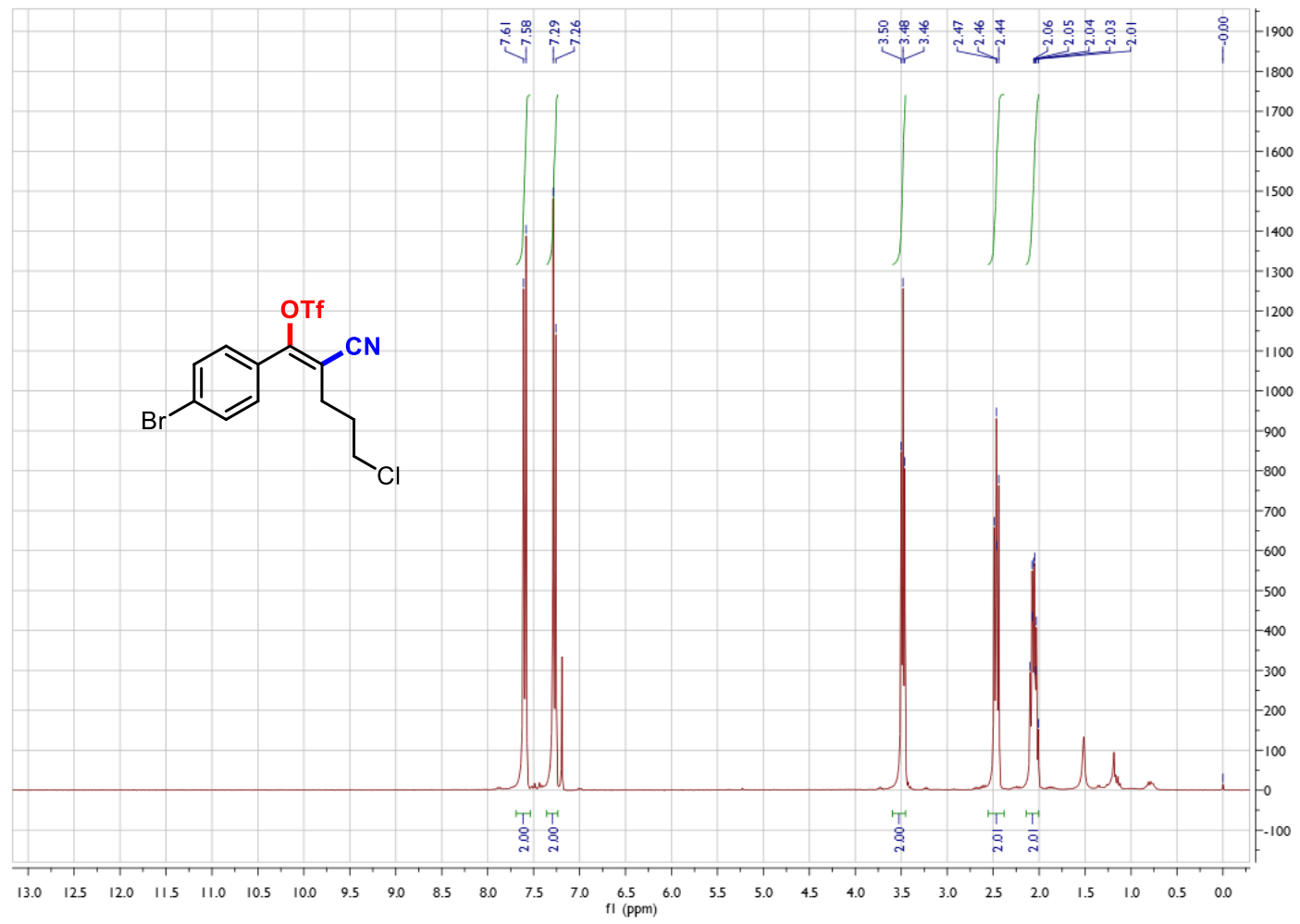

${ }^{13}$ C NMR Spectrum of (Z)-1-(4-bromophenyl)-5-chloro-2-cyanopent-1-en-1-yl trifluoromethanesulfonate $2 q$

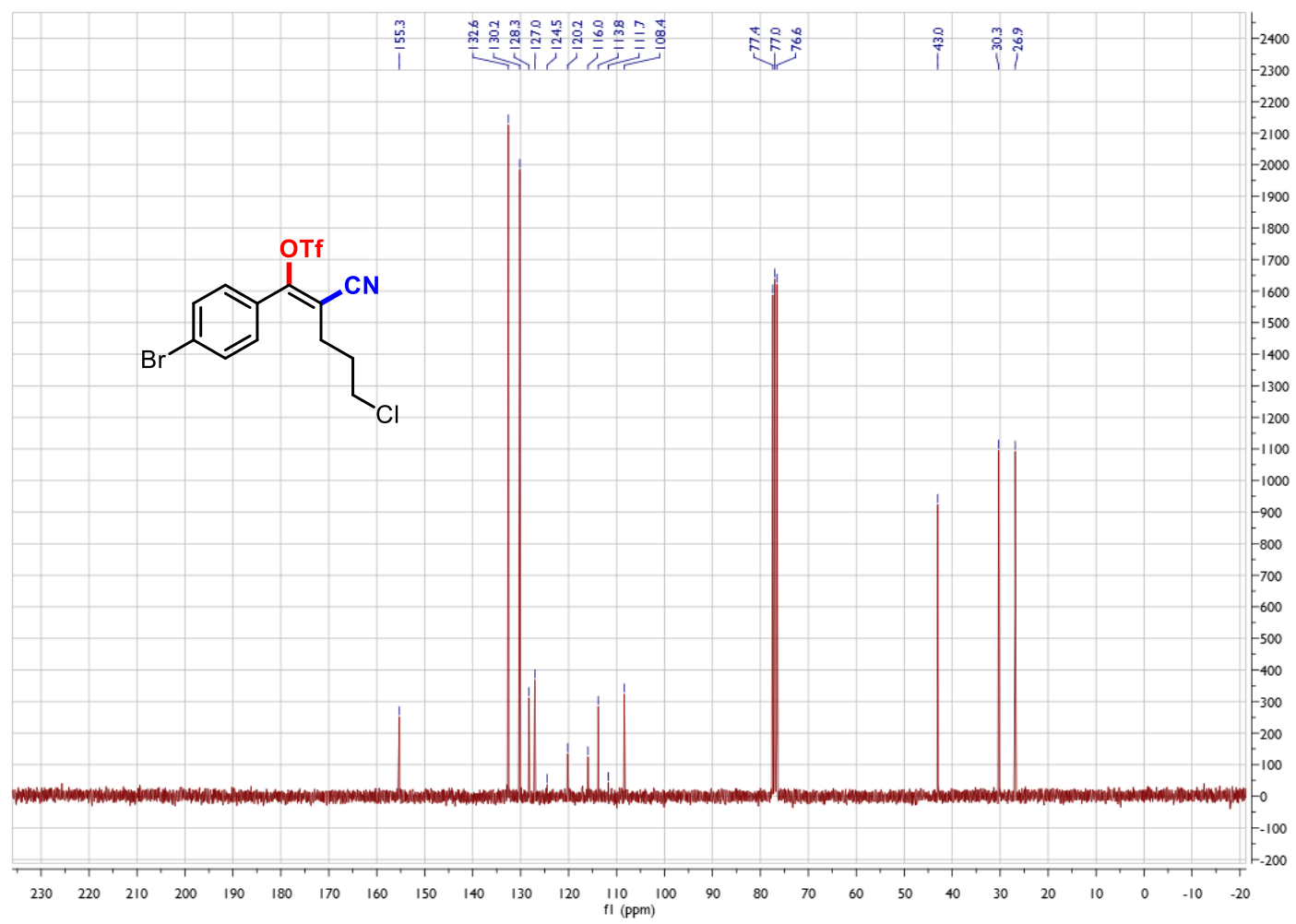


${ }^{19}$ F NMR Spectrum of (Z)-1-(4-bromophenyl)-5-chloro-2-cyanopent-1-en-1-yl trifluoromethanesulfonate $2 q$

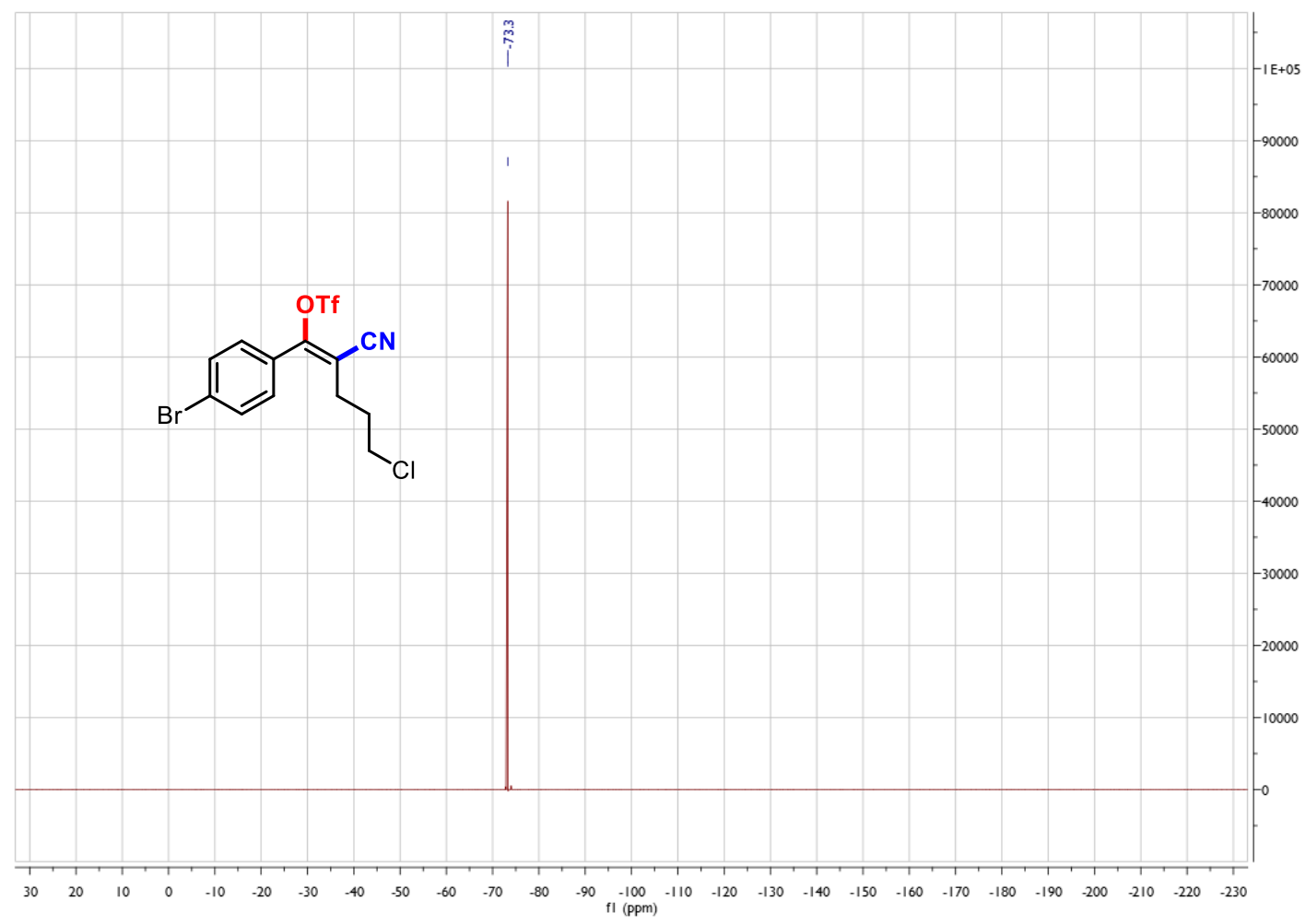


${ }^{1}$ H NMR Spectrum of (Z)-4-(4-bromophenyl)-3-cyano-4-(((trifluoromethyl)sulfonyl)-oxy)but-3-en-1-yl 4-methylbenzenesulfonate $2 r$

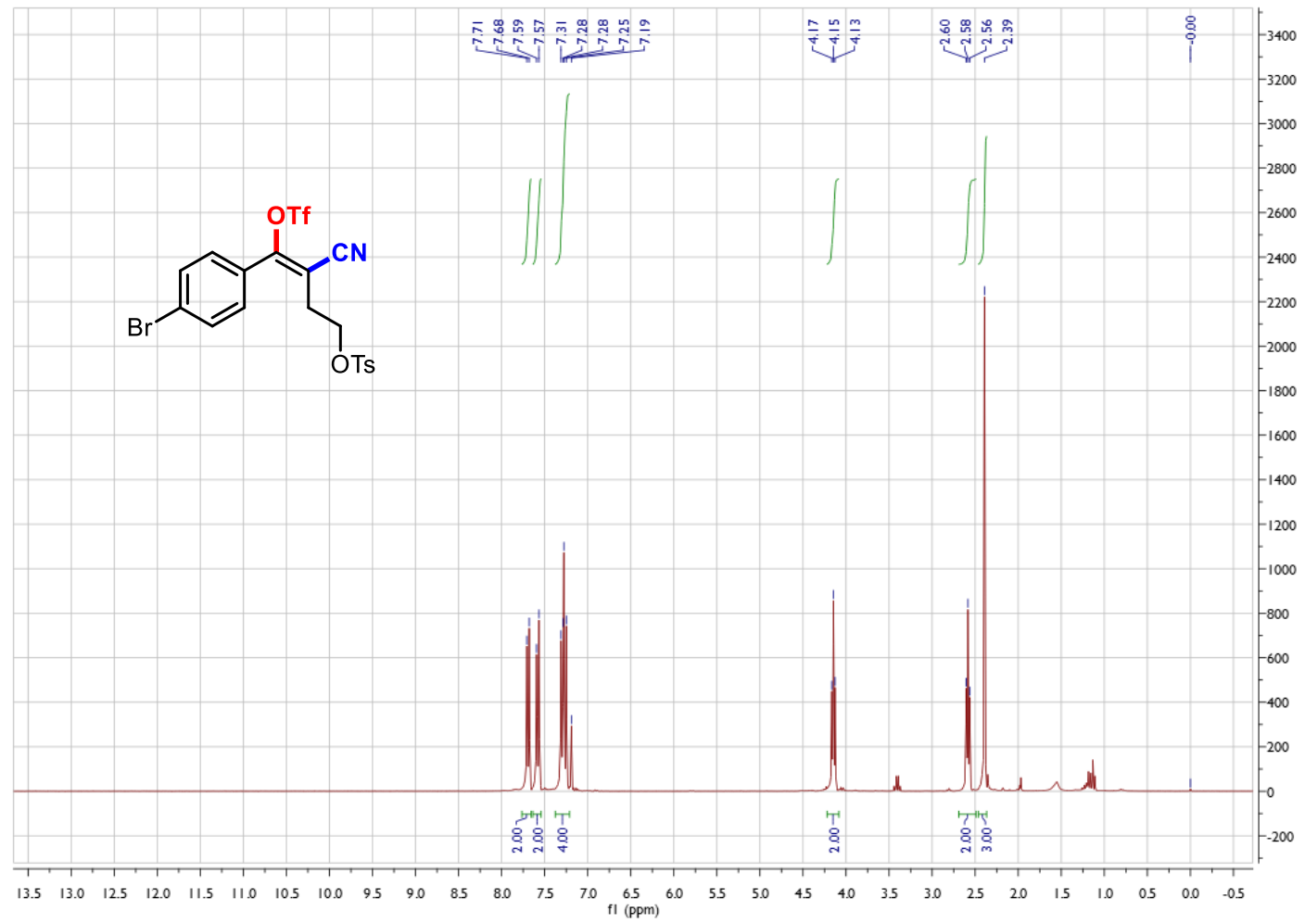

${ }^{13} \mathrm{C}$ NMR Spectrum of (Z)-4-(4-bromophenyl)-3-cyano-4-(((trifluoromethyl)sulfonyl)-oxy)but-3-en-1-yl 4-methylbenzenesulfonate $2 \mathrm{r}$

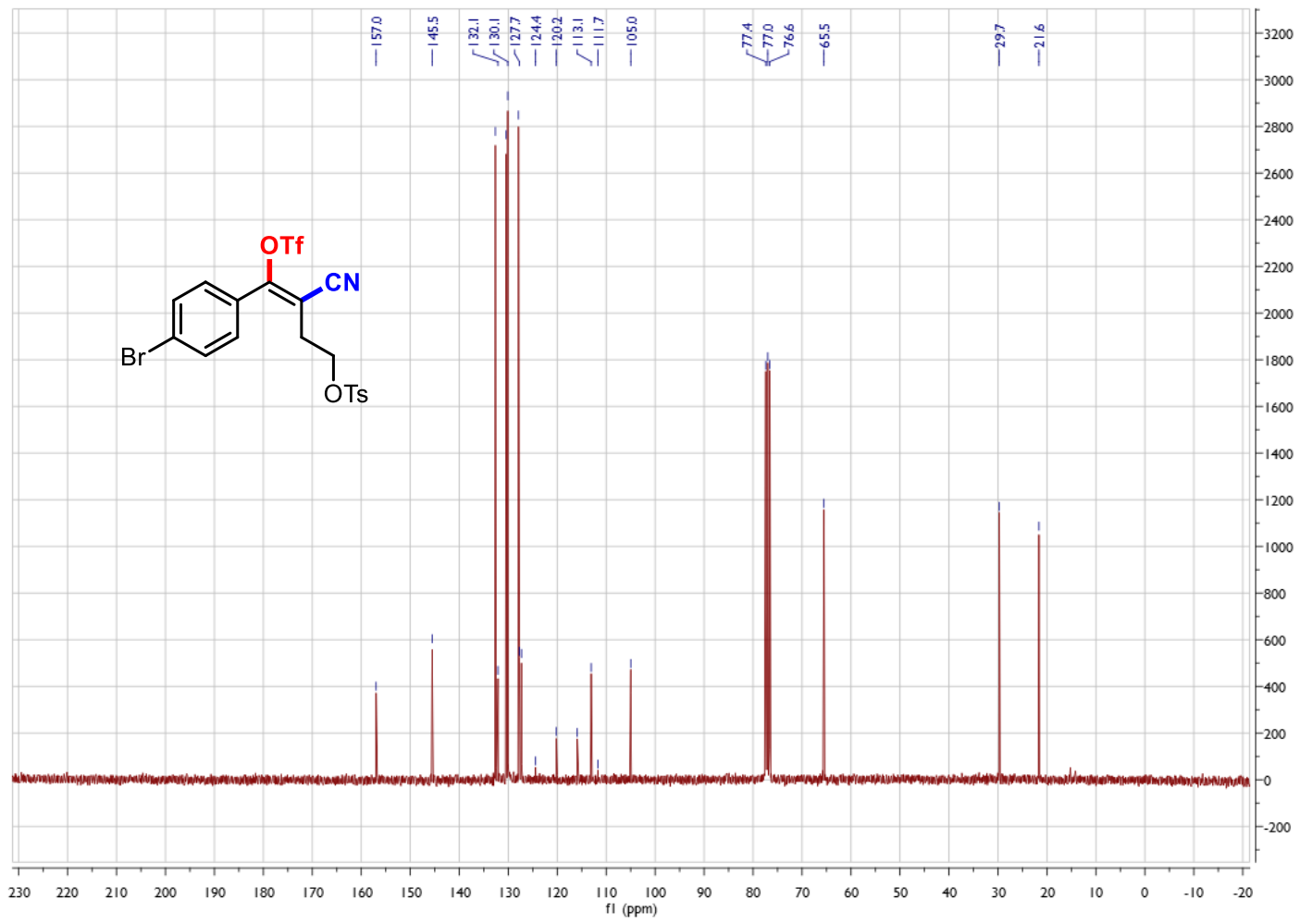


${ }^{19}$ F NMR Spectrum of (Z)-4-(4-bromophenyl)-3-cyano-4-(((trifluoromethyl)sulfonyl)-oxy)but-3-en-1-yl 4-methylbenzenesulfonate $2 r$

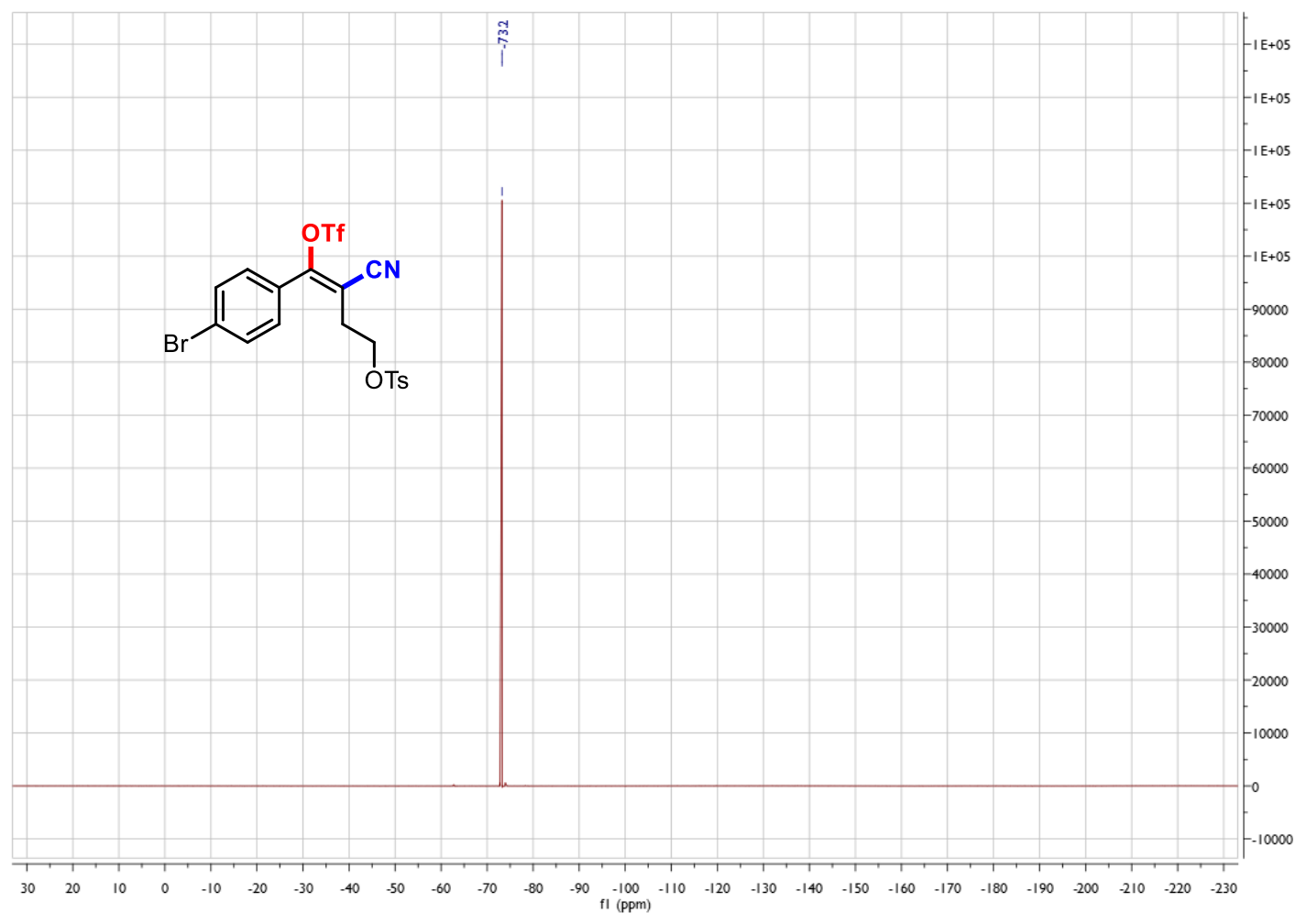


${ }^{1} \mathrm{H}$ NMR Spectrum of (Z)-1-(4-bromophenyl)-2-cyano-6-(1,3-dioxoisoindolin-2yl)hex-1-en-1-yl trifluoromethanesulfonate $2 \mathrm{~s}$

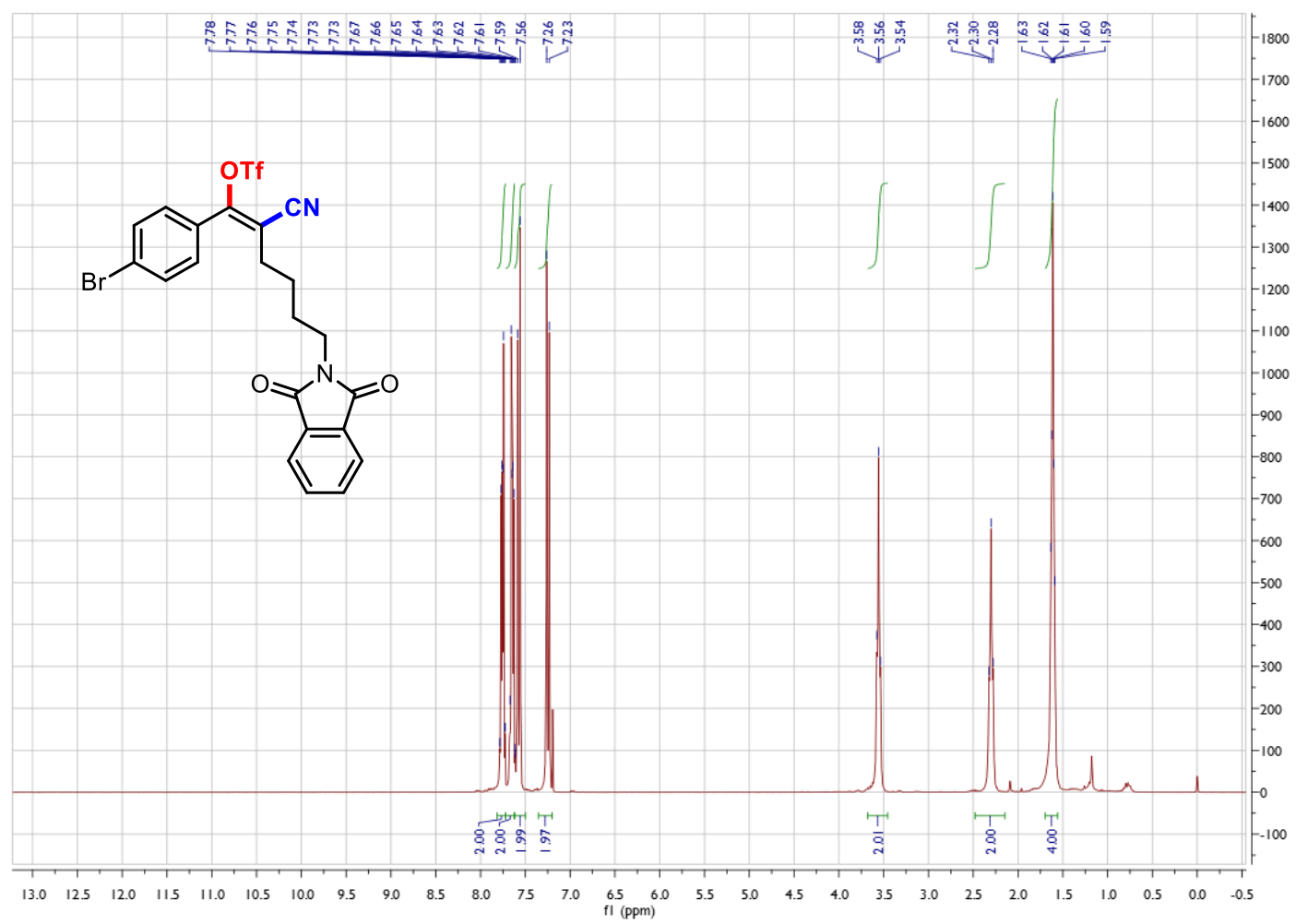

${ }^{13}$ C NMR Spectrum of (Z)-1-(4-bromophenyl)-2-cyano-6-(1,3-dioxoisoindolin-2yl)hex-1-en-1-yl trifluoromethanesulfonate $2 \mathrm{~s}$

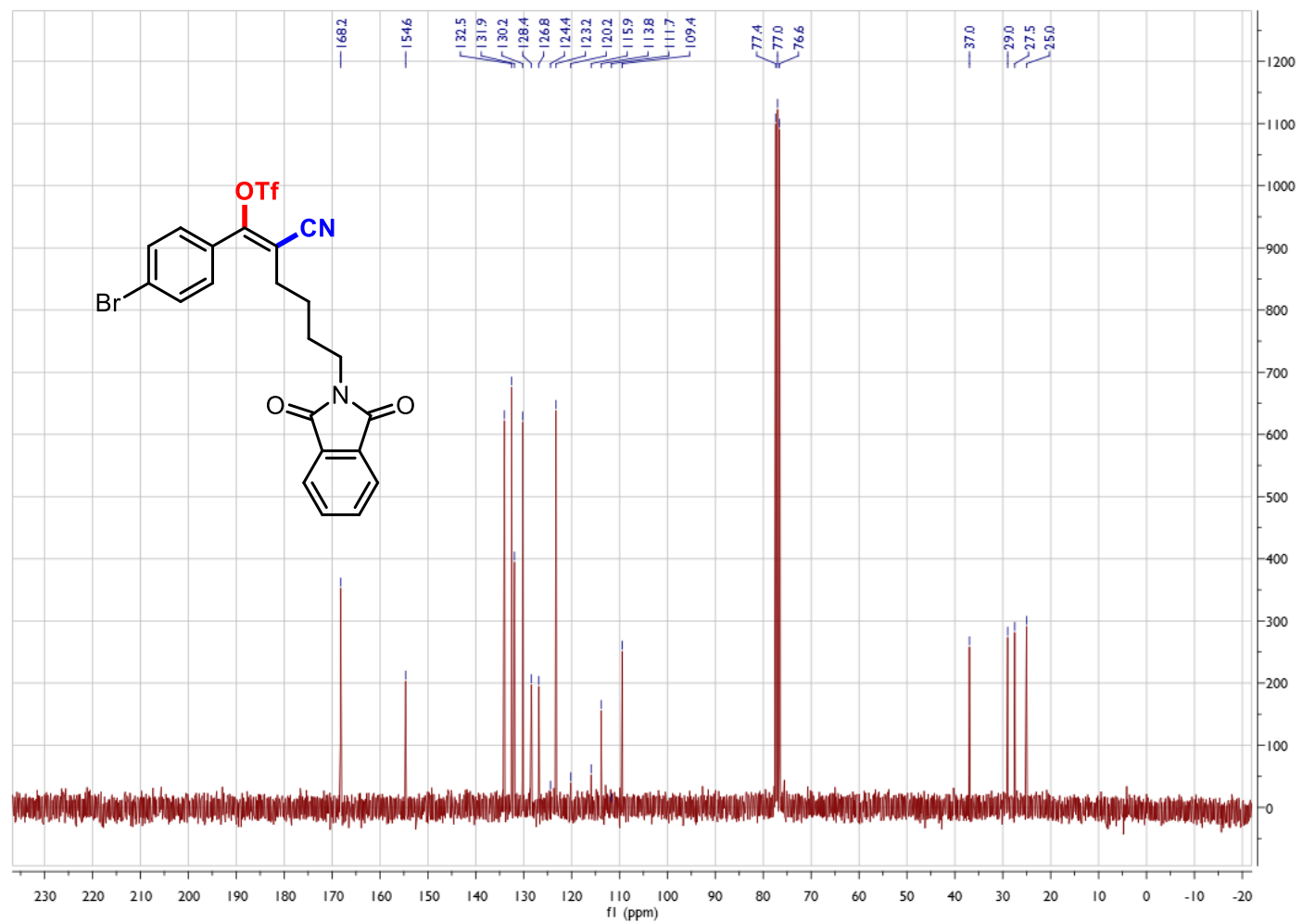


${ }^{19}$ F NMR Spectrum of (Z)-1-(4-bromophenyl)-2-cyano-6-(1,3-dioxoisoindolin-2yl)hex-1-en-1-yl trifluoromethanesulfonate $2 \mathrm{~s}$

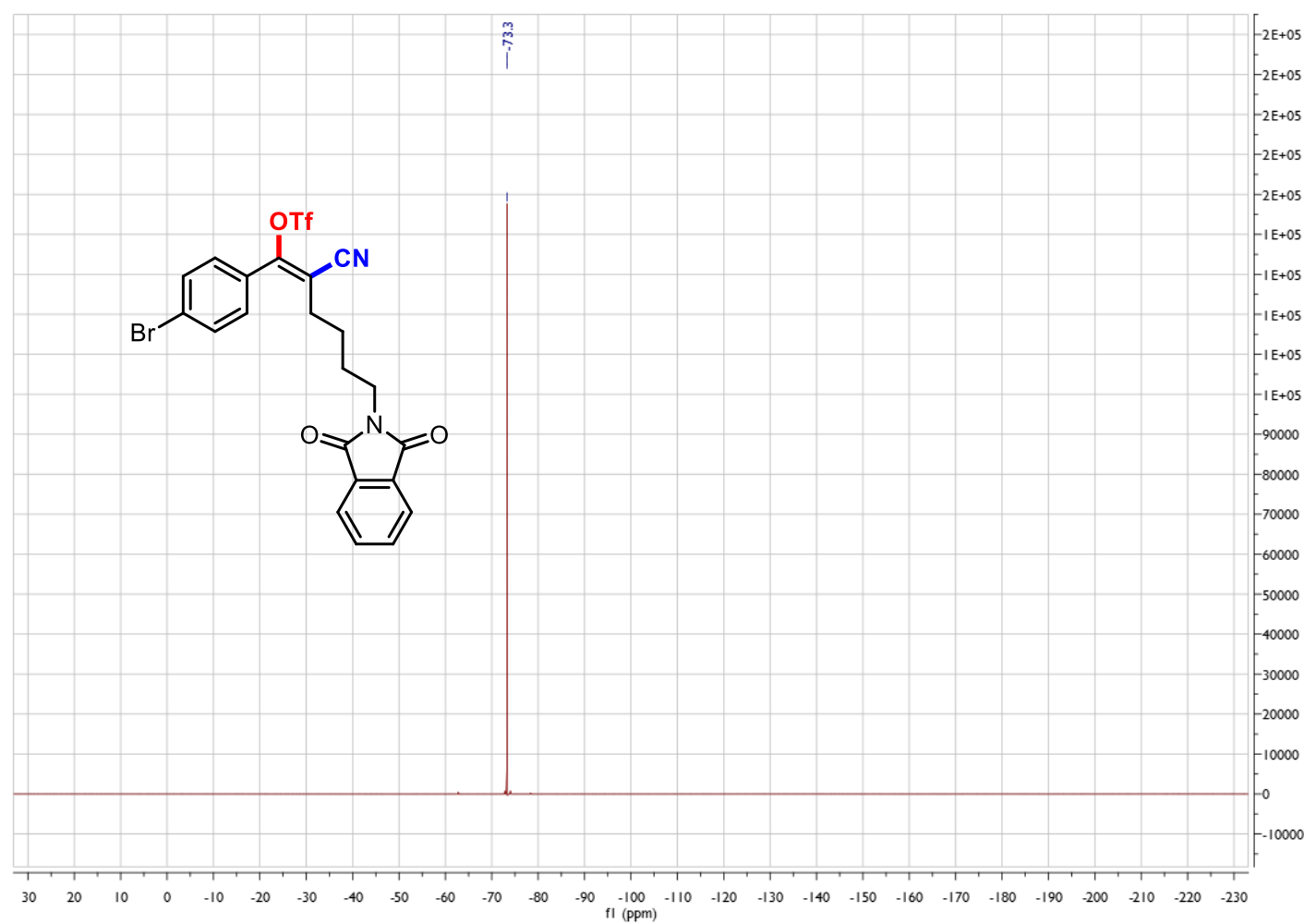


${ }^{1}$ H NMR Spectrum of (Z)-1-(4-bromophenyl)-2-cyanoprop-1-en-1-yl trifluoromethanesulfonate $2 \mathrm{t}$

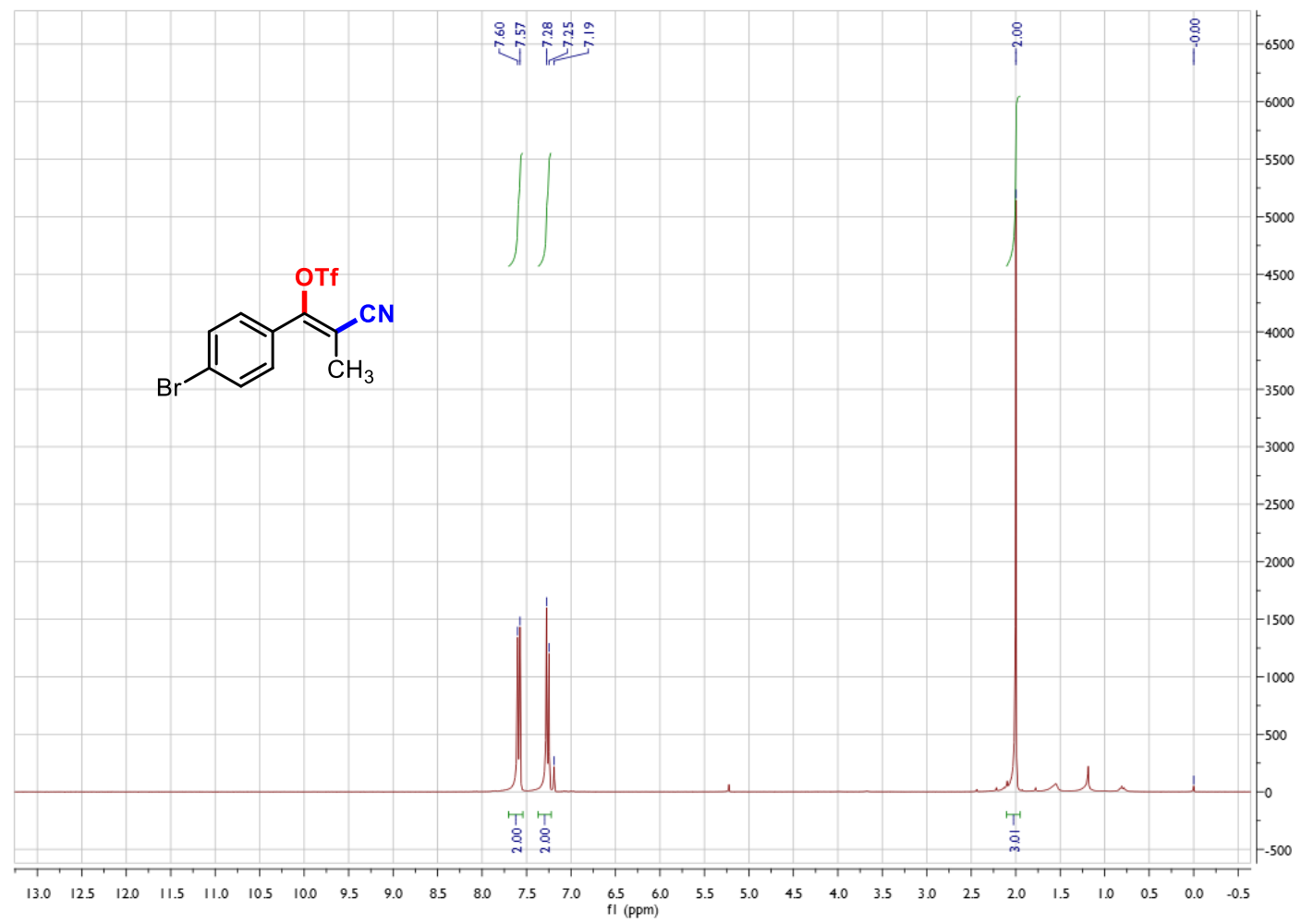

${ }^{13}$ C NMR Spectrum of (Z)-1-(4-bromophenyl)-2-cyanoprop-1-en-1-yl trifluoromethanesulfonate $2 t$

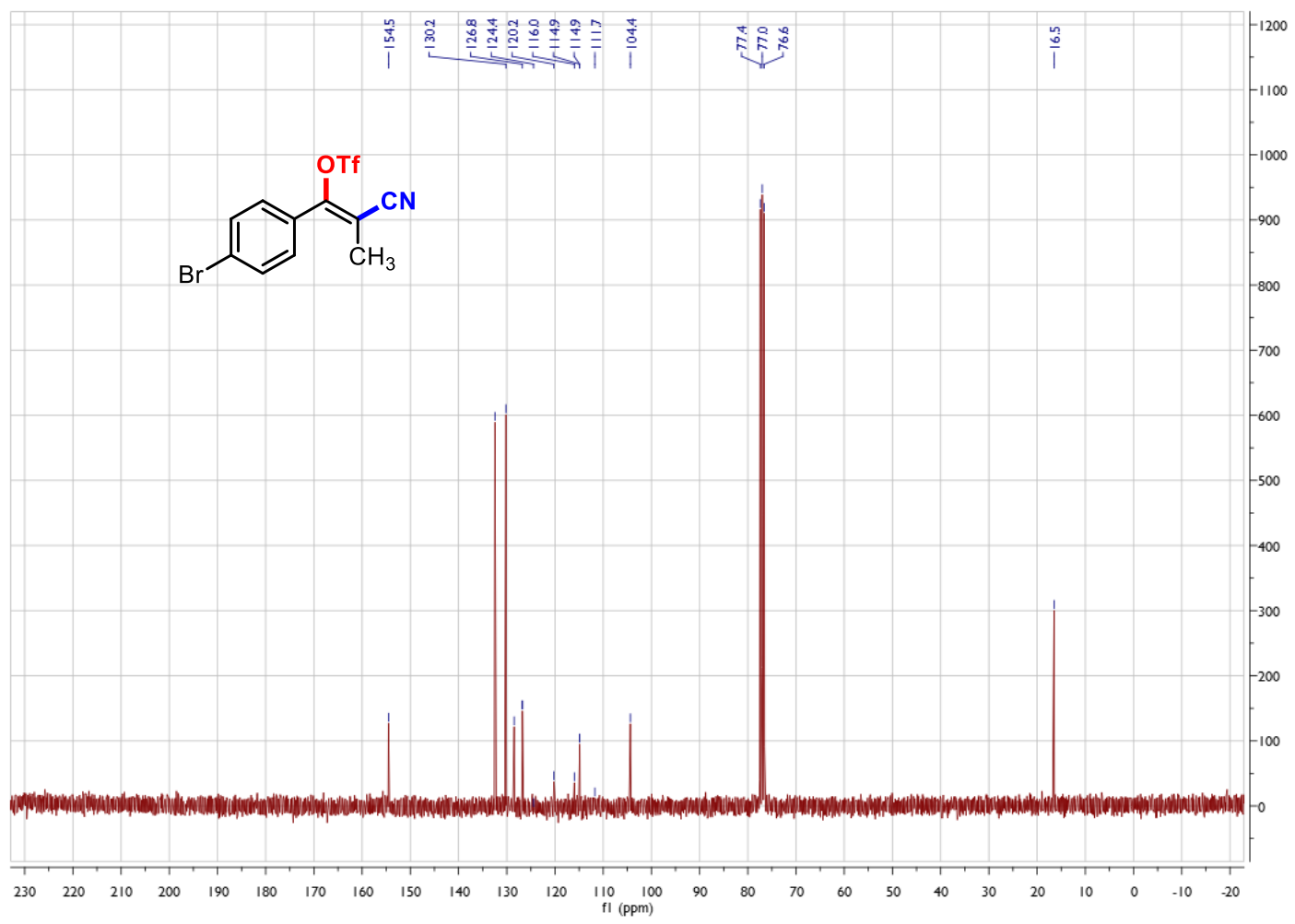


${ }^{19}$ F NMR Spectrum of (Z)-1-(4-bromophenyl)-2-cyanoprop-1-en-1-yl trifluoromethanesulfonate $2 t$

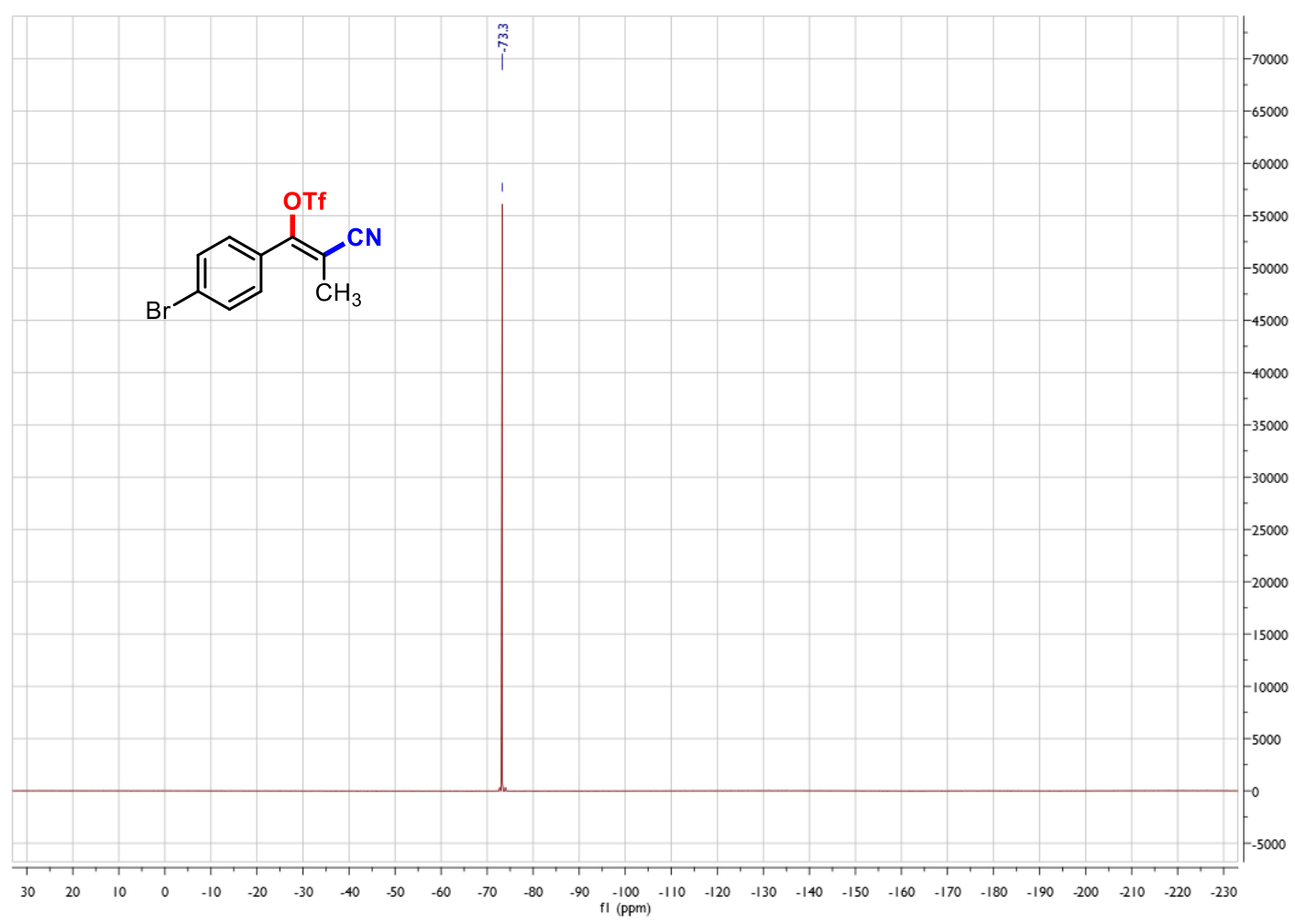


${ }^{1}$ H NMR Spectrum of (Z)-1-(4-bromophenyl)-2-cyano-3-methylbut-1-en-1-yl trifluoromethanesulfonate $2 u$

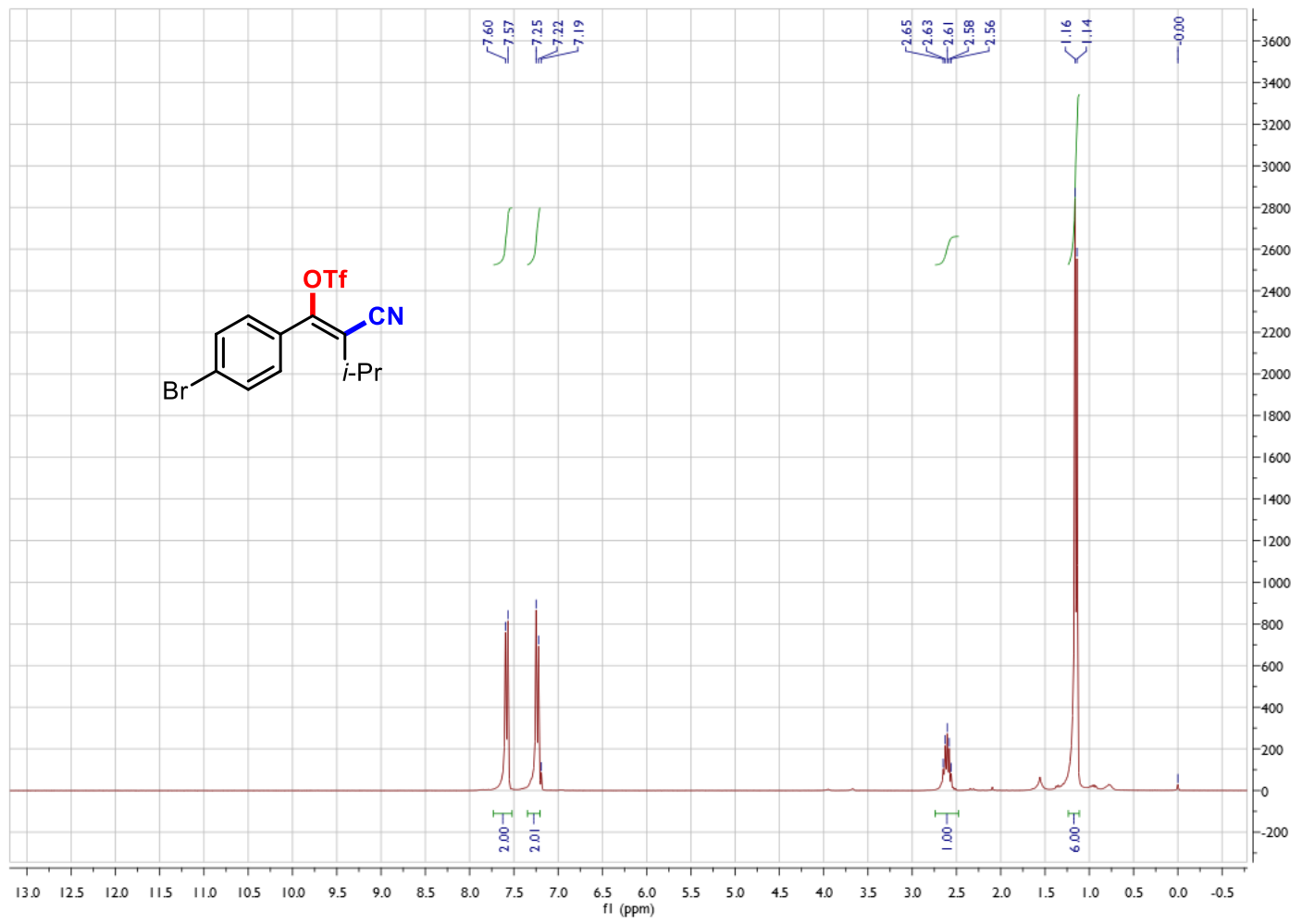

${ }^{13}$ C NMR Spectrum of (Z)-1-(4-bromophenyl)-2-cyano-3-methylbut-1-en-1-yl trifluoromethanesulfonate $2 u$

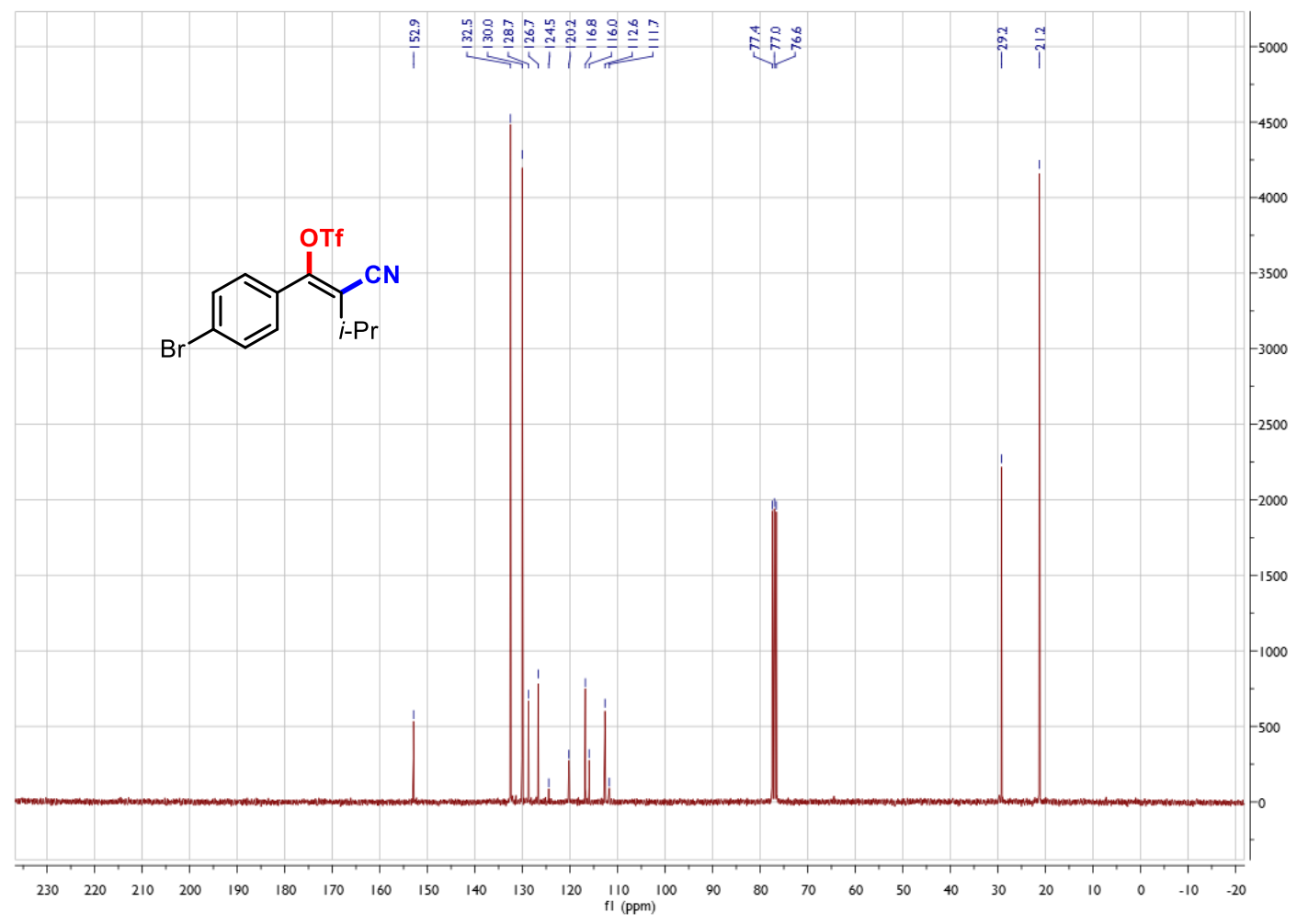


${ }^{19}$ F NMR Spectrum of (Z)-1-(4-bromophenyl)-2-cyano-3-methylbut-1-en-1-yl trifluoromethanesulfonate $2 u$

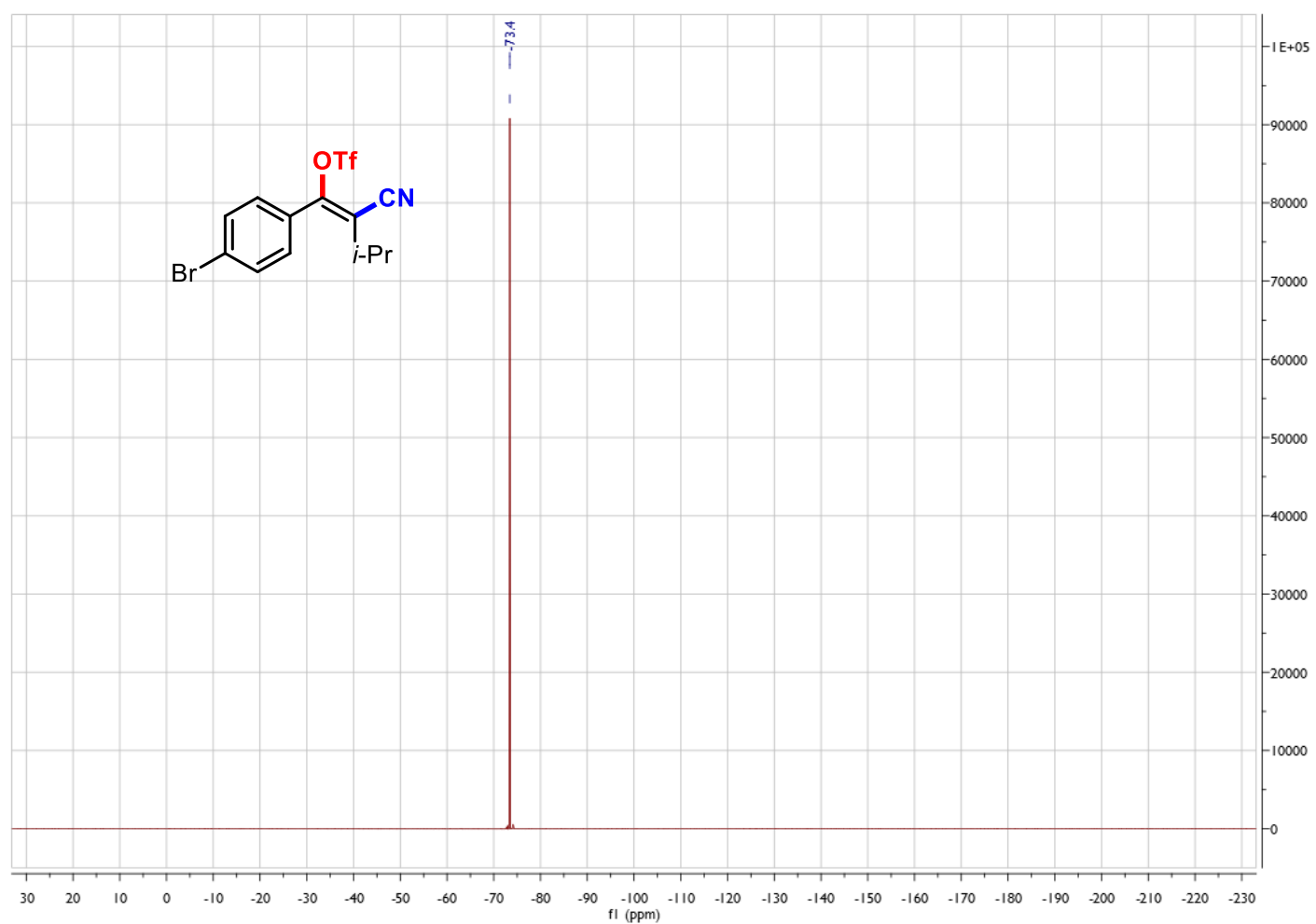


${ }^{1}$ H NMR Spectrum of (Z)-1-(4-bromophenyl)-2-cyano-3,3-dimethylbut-1-en-1-yl trifluoromethanesulfonate $2 \mathrm{v}$

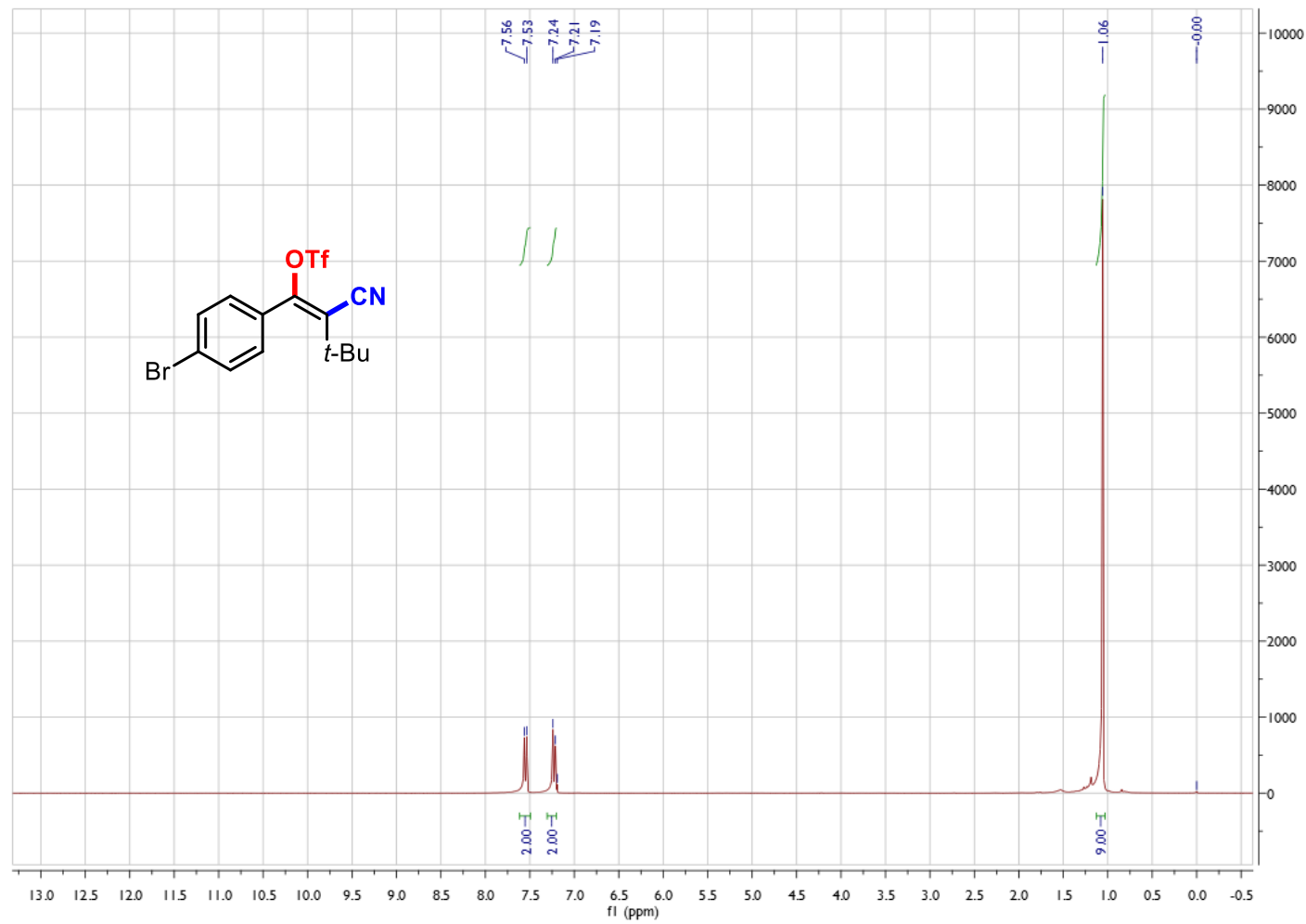

${ }^{13} \mathrm{C}$ NMR Spectrum of (Z)-1-(4-bromophenyl)-2-cyano-3,3-dimethylbut-1-en-1-yl trifluoromethanesulfonate $2 \mathrm{v}$

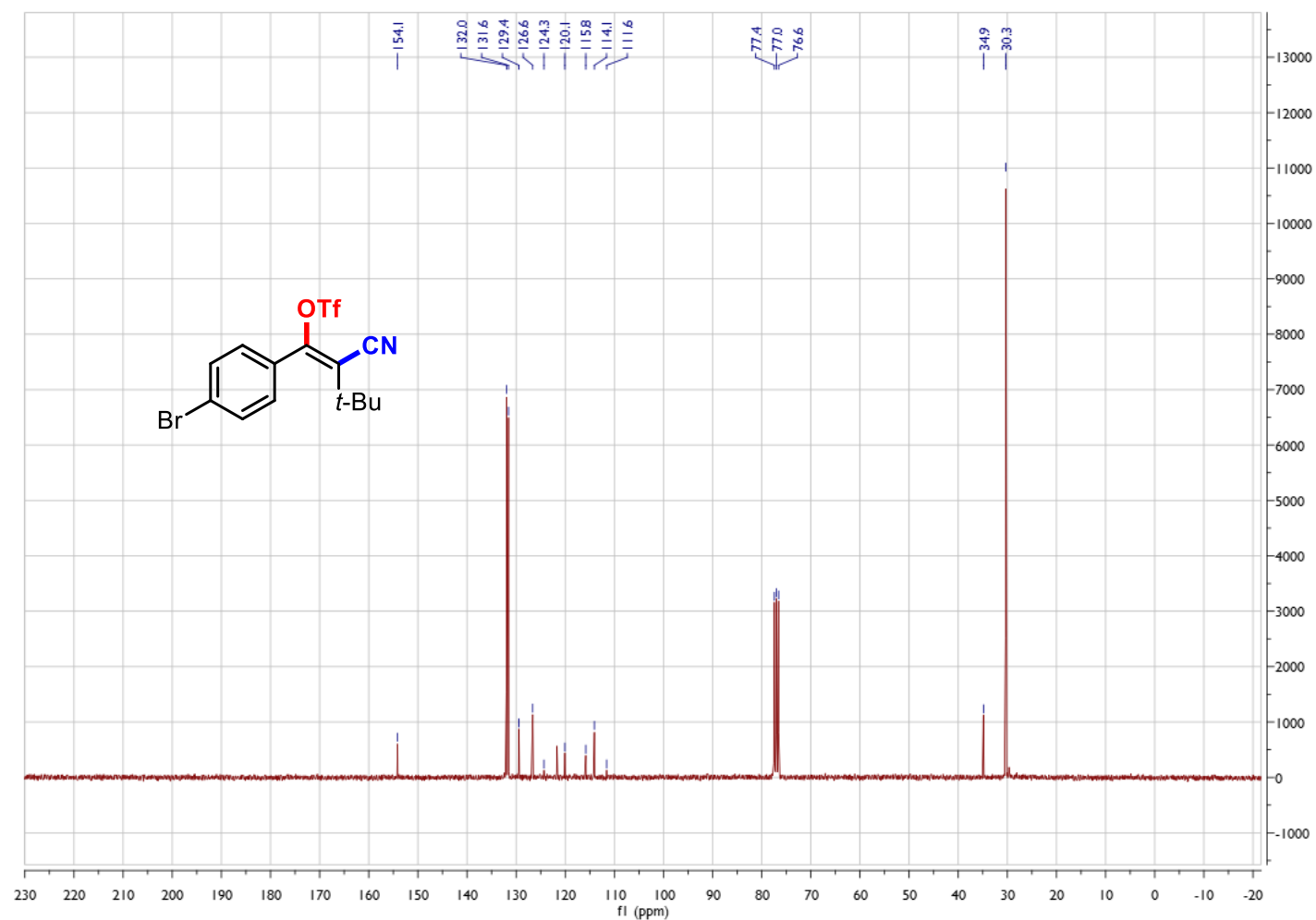


${ }^{19}$ F NMR Spectrum of (Z)-1-(4-bromophenyl)-2-cyano-3, 3-dimethylbut-1-en-1-yl trifluoromethanesulfonate $2 \mathrm{v}$

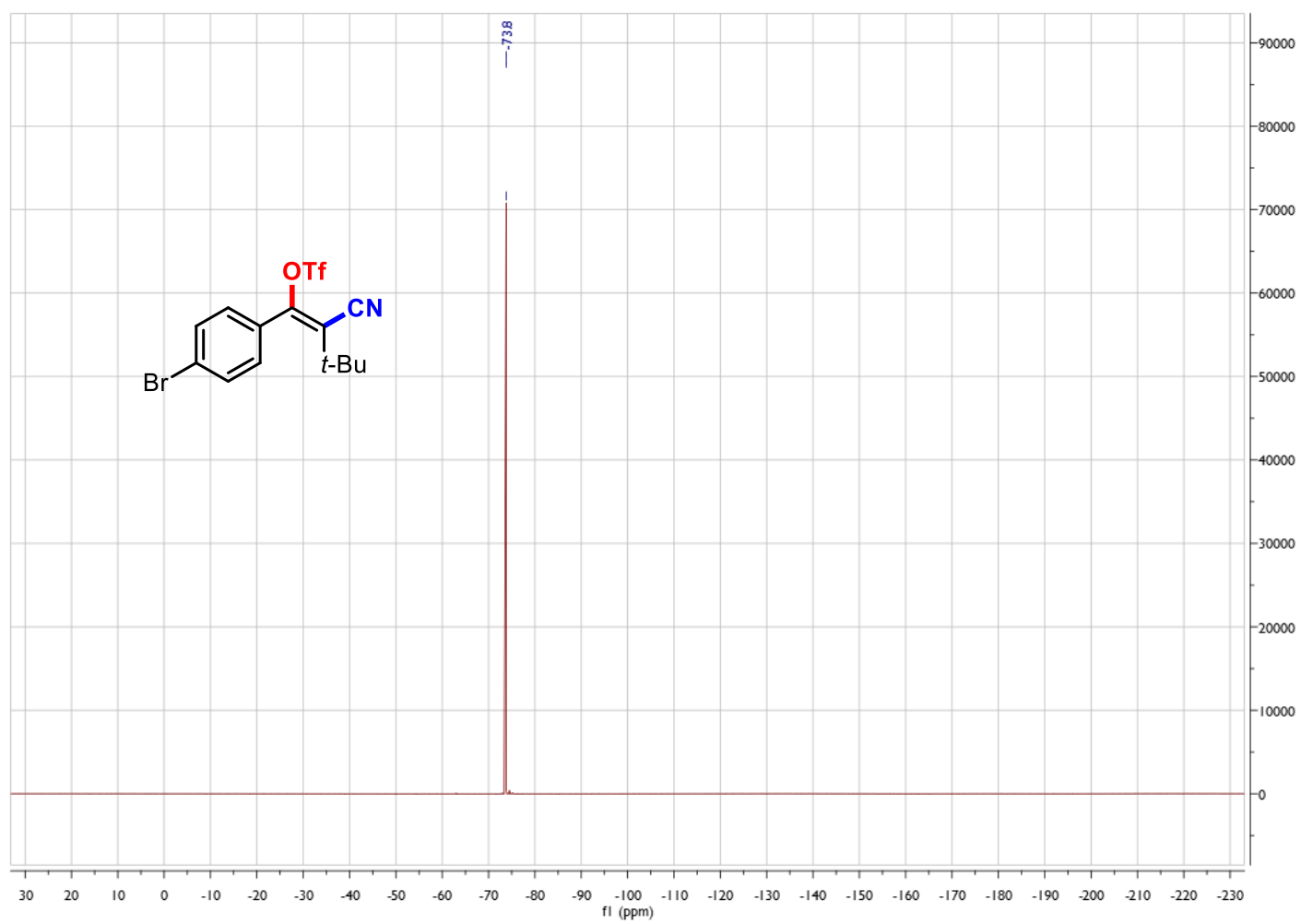


${ }^{1} \mathrm{H}$ NMR Spectrum of (Z)-1-(4-bromophenyl)-2-cyano-2-cyclopropylvinyl trifluoromethanesulfonate $2 \mathrm{w}$

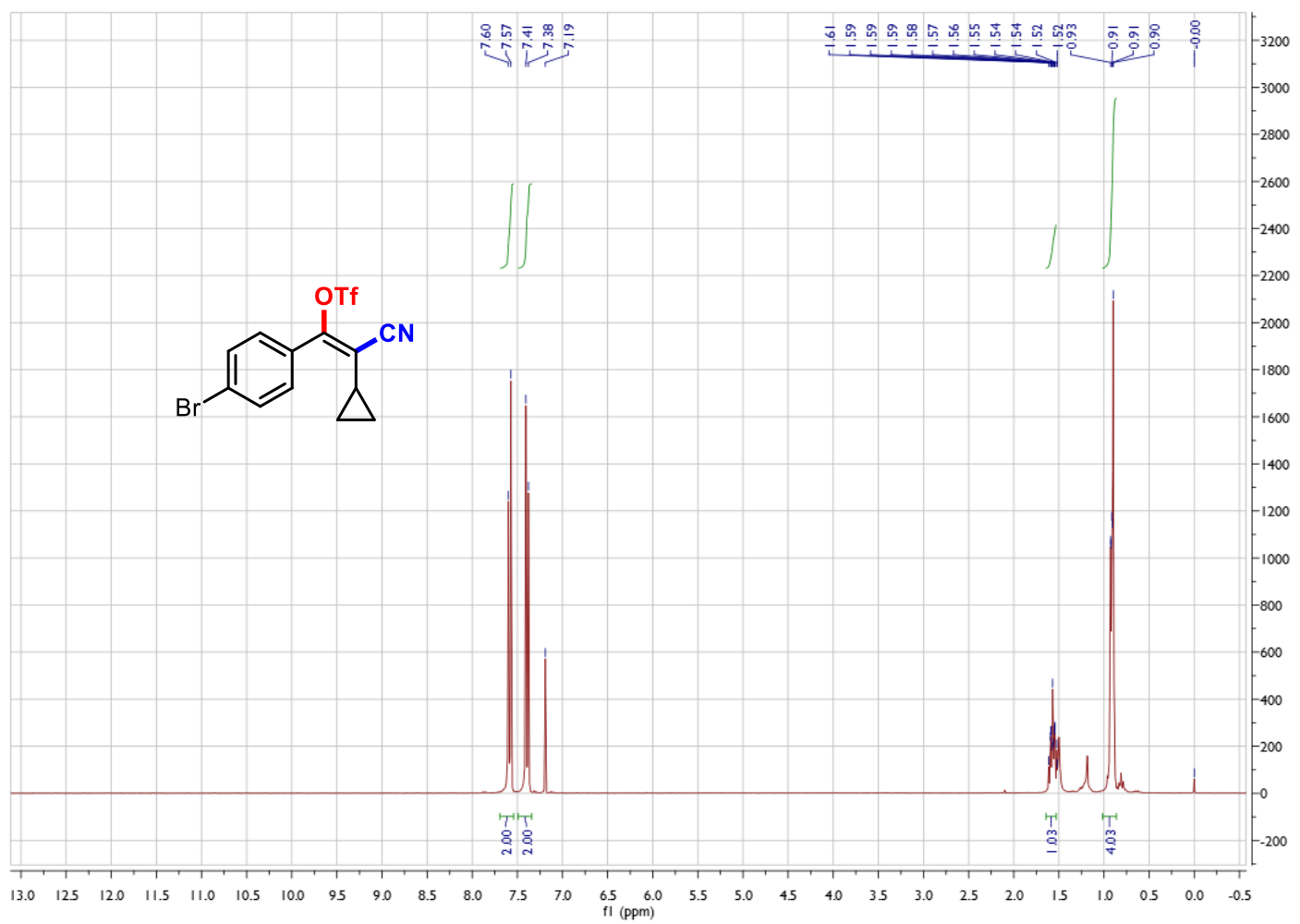

${ }^{13} \mathrm{C}$ NMR Spectrum of (Z)-1-(4-bromophenyl)-2-cyano-2-cyclopropylvinyl trifluoromethanesulfonate $2 \mathrm{w}$

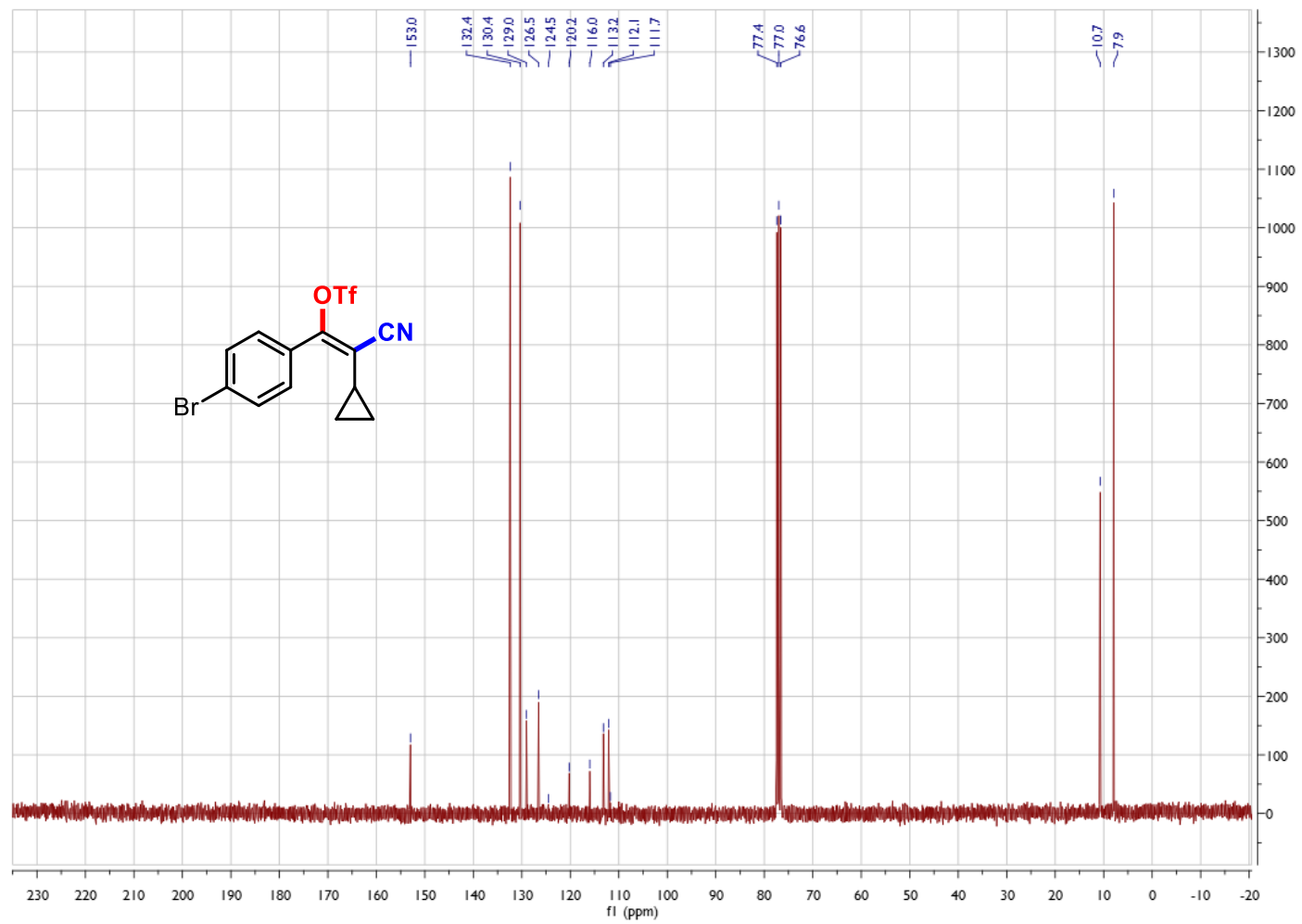


${ }^{19} \mathrm{~F}$ NMR Spectrum of (Z)-1-(4-bromophenyl)-2-cyano-2-cyclopropylvinyl trifluoromethanesulfonate $\mathbf{2 w}$

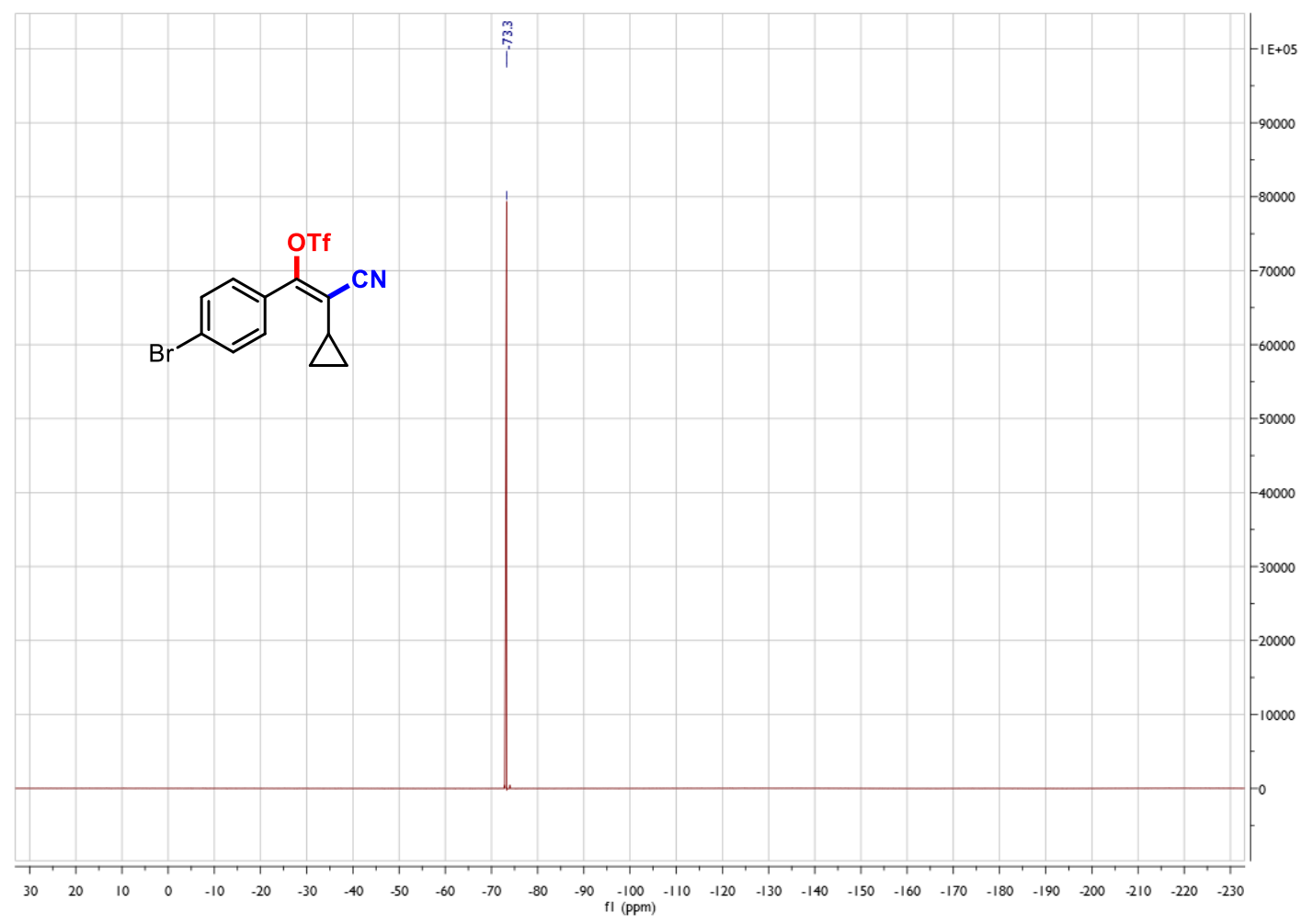


${ }^{1} \mathrm{H}$ NMR Spectrum of (Z)-1-(4-bromophenyl)-2-cyano-2-cyclopentylvinyl trifluoromethanesulfonate $2 x$

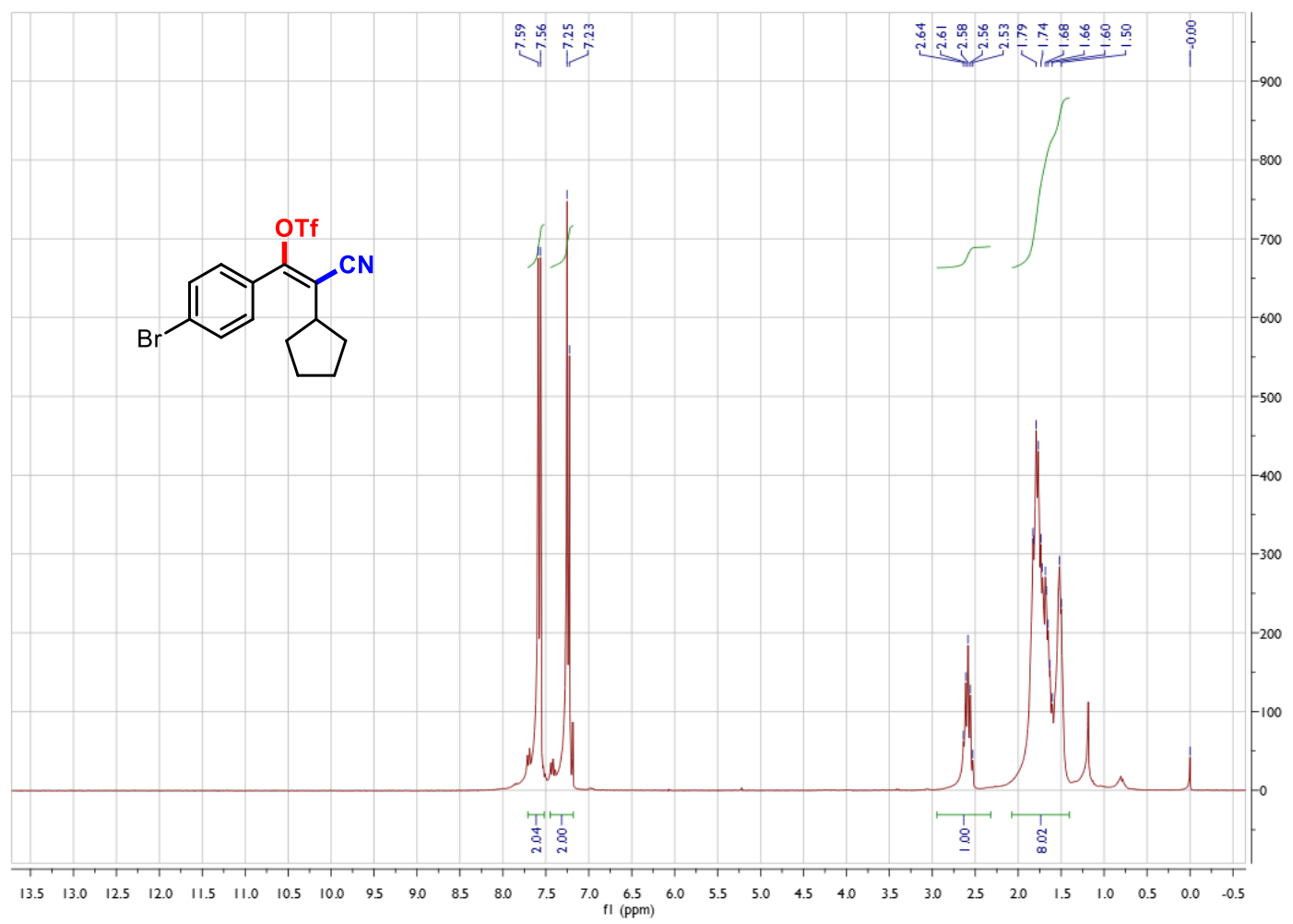

${ }^{13} \mathrm{C}$ NMR Spectrum of (Z)-1-(4-bromophenyl)-2-cyano-2-cyclopentylvinyl trifluoromethanesulfonate $2 \mathrm{x}$

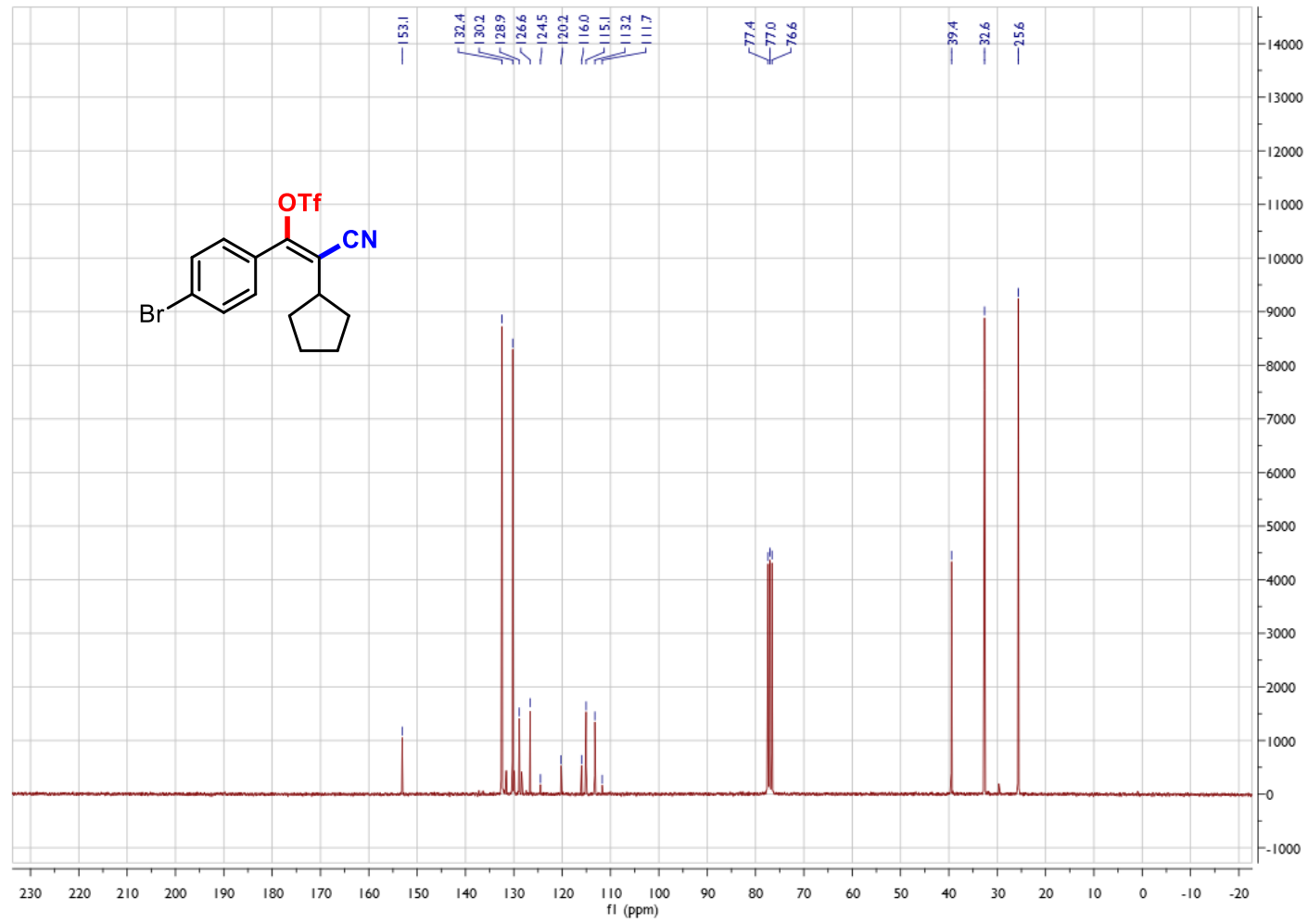


${ }^{19}$ F NMR Spectrum of (Z)-1-(4-bromophenyl)-2-cyano-2-cyclopentylvinyl trifluoromethanesulfonate $2 x$

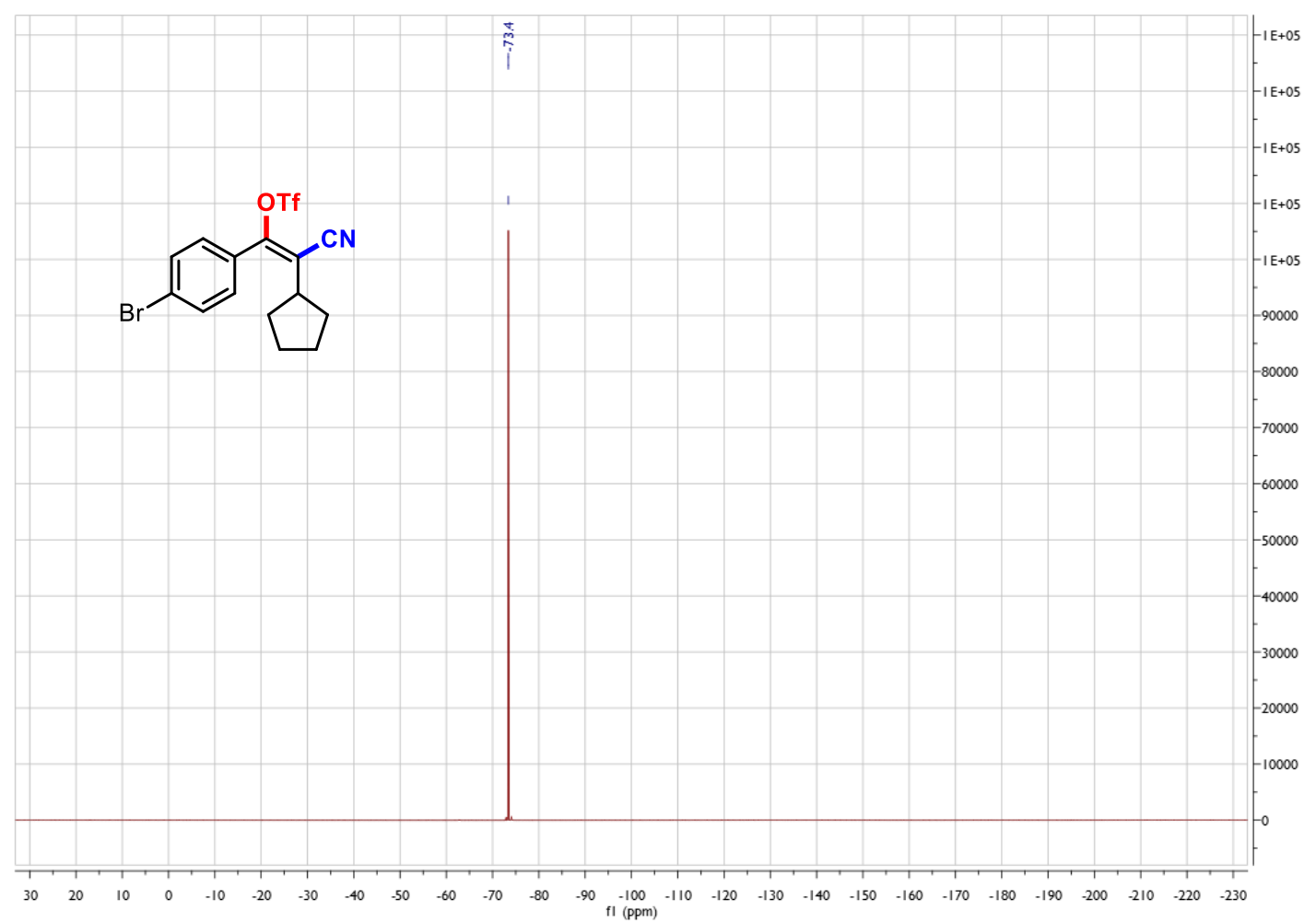


${ }^{1} \mathrm{H}$ NMR Spectrum of (Z)-1-(4-bromophenyl)-2-cyano-2-cyclohexylvinyl trifluoromethanesulfonate $2 y$

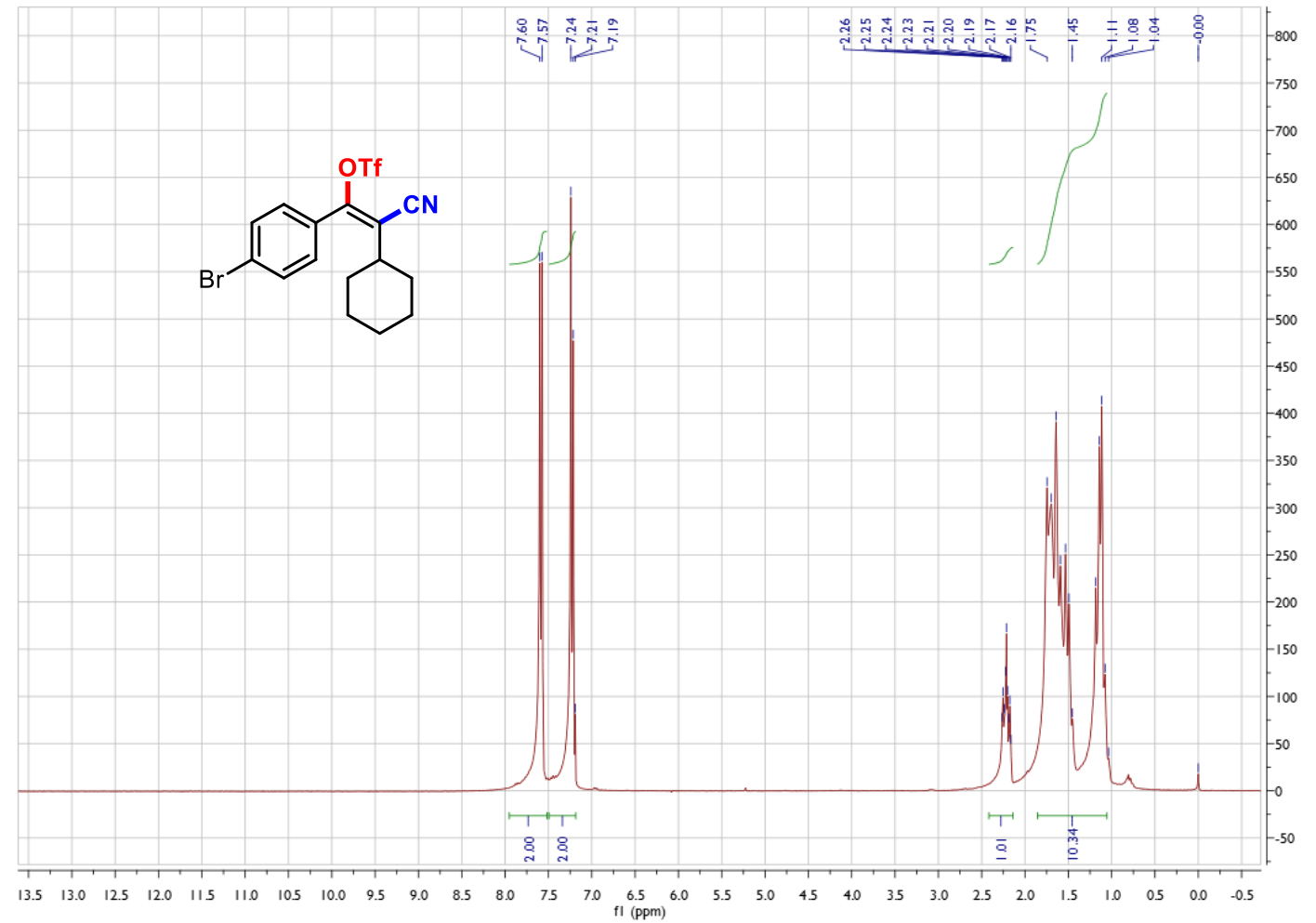

${ }^{13} \mathrm{C}$ NMR Spectrum of (Z)-1-(4-bromophenyl)-2-cyano-2-cyclohexylvinyl trifluoromethanesulfonate $2 \mathrm{y}$

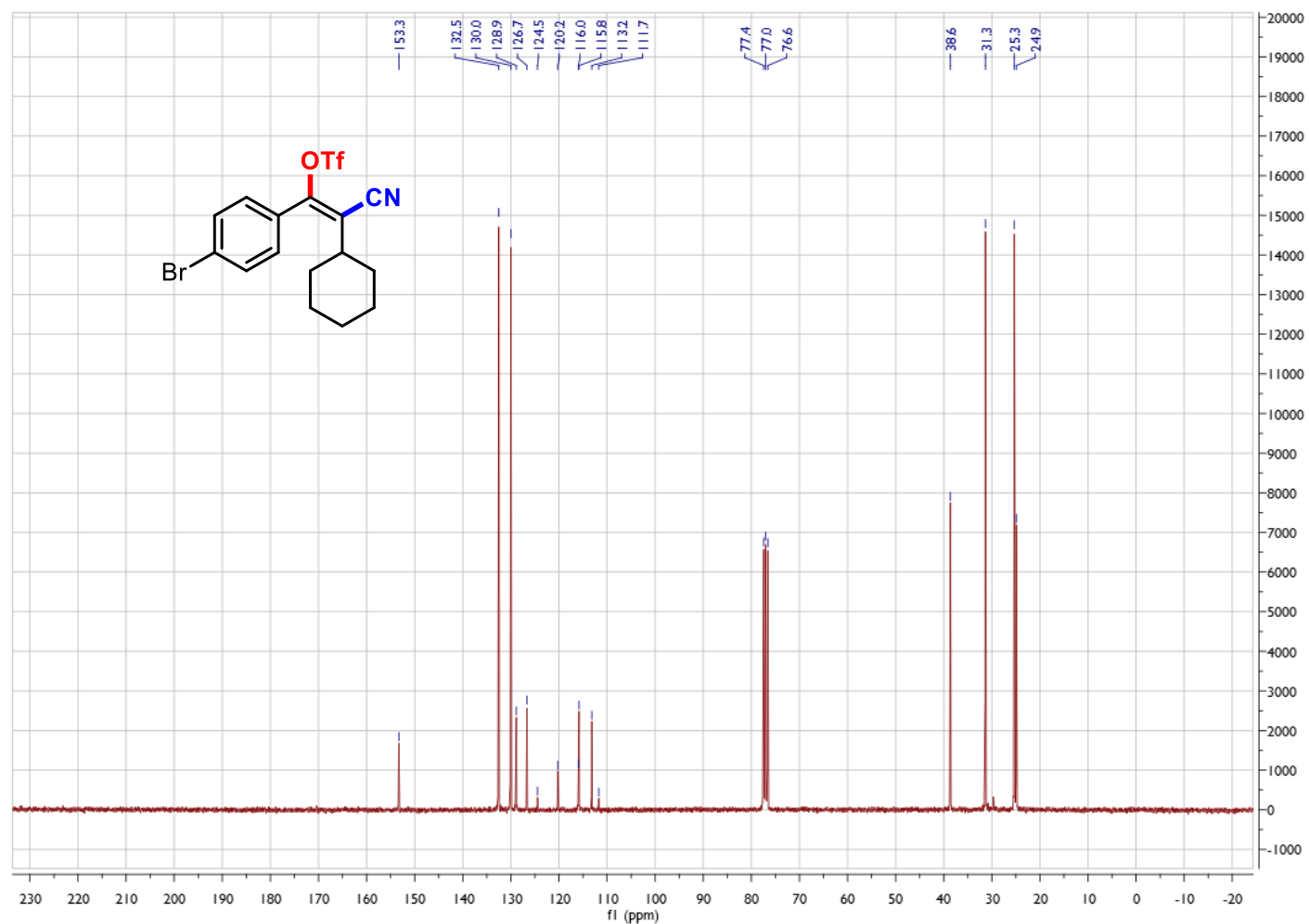


${ }^{19} \mathrm{~F}$ NMR Spectrum of (Z)-1-(4-bromophenyl)-2-cyano-2-cyclohexylvinyl trifluoromethanesulfonate $2 \mathrm{y}$

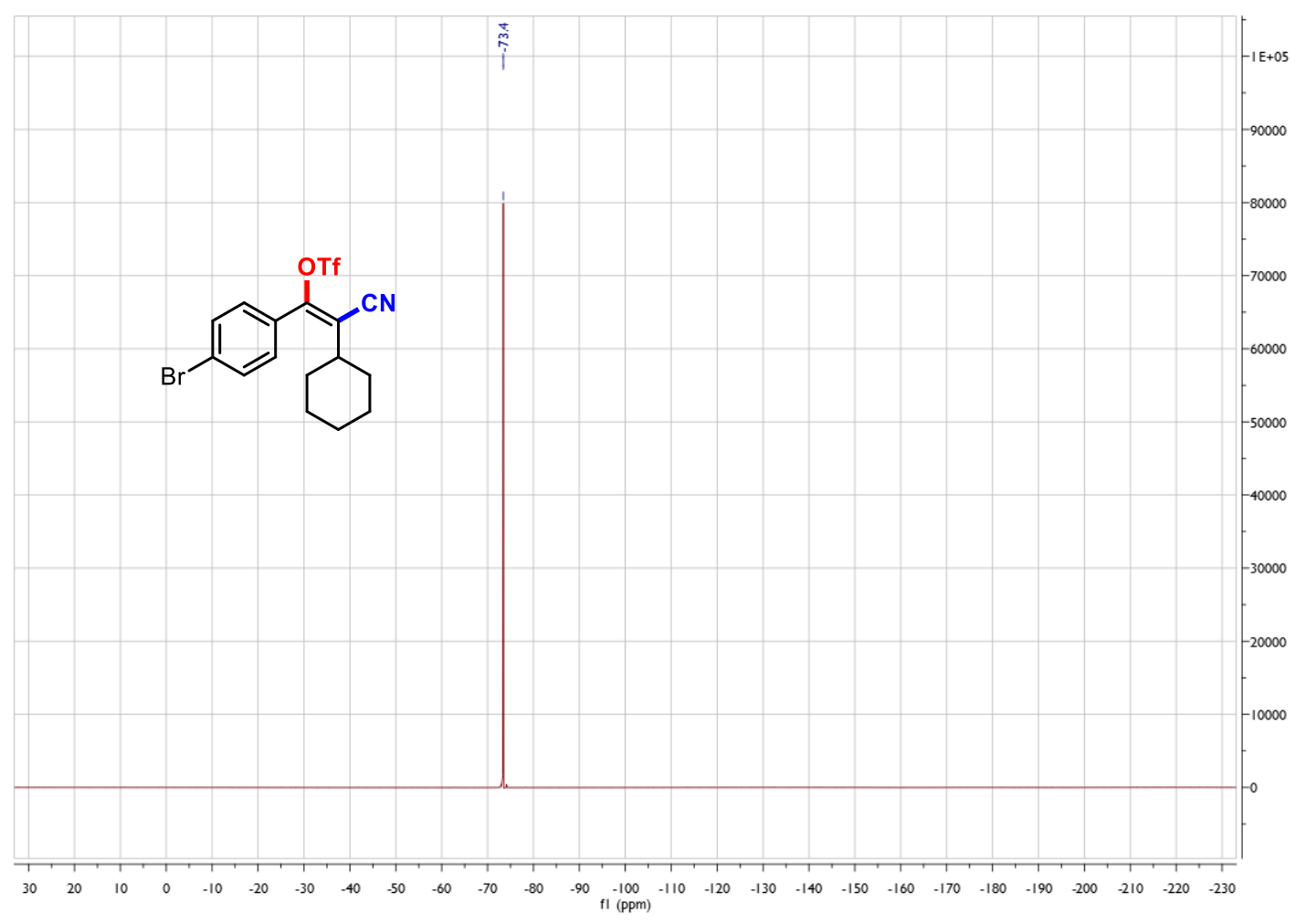


${ }^{1}$ H NMR Spectrum of (E)-(4-chlorophenyl)(2-(4-chlorophenyl)-3-cyanocyclopent2-en-1-ylidene)methyl trifluoromethanesulfonate $2 z$

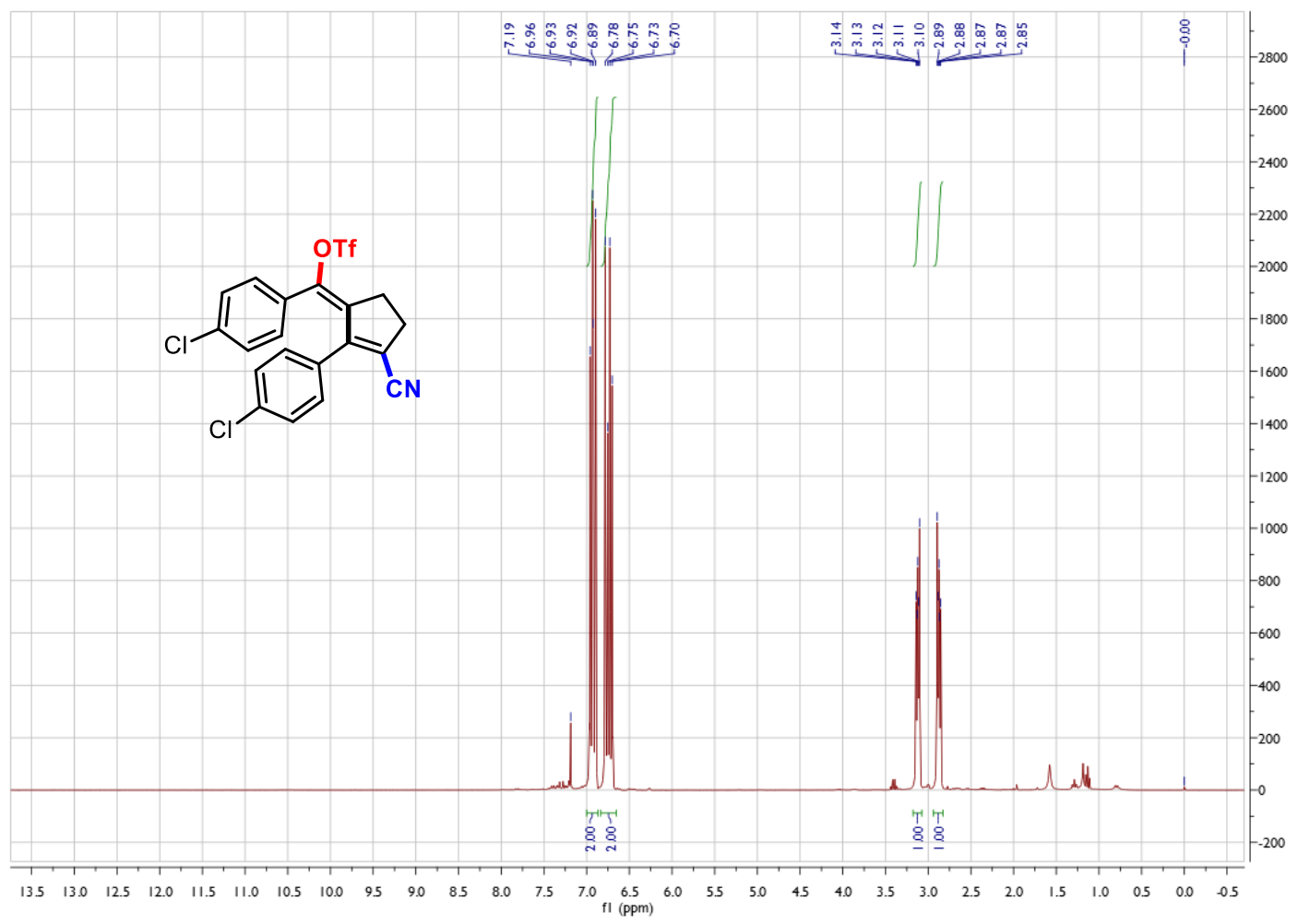

${ }^{13}$ C NMR Spectrum of (E)-(4-chlorophenyl)(2-(4-chlorophenyl)-3-cyanocyclopent - 2-en-1-ylidene)methyl trifluoromethanesulfonate $2 \mathrm{z}$

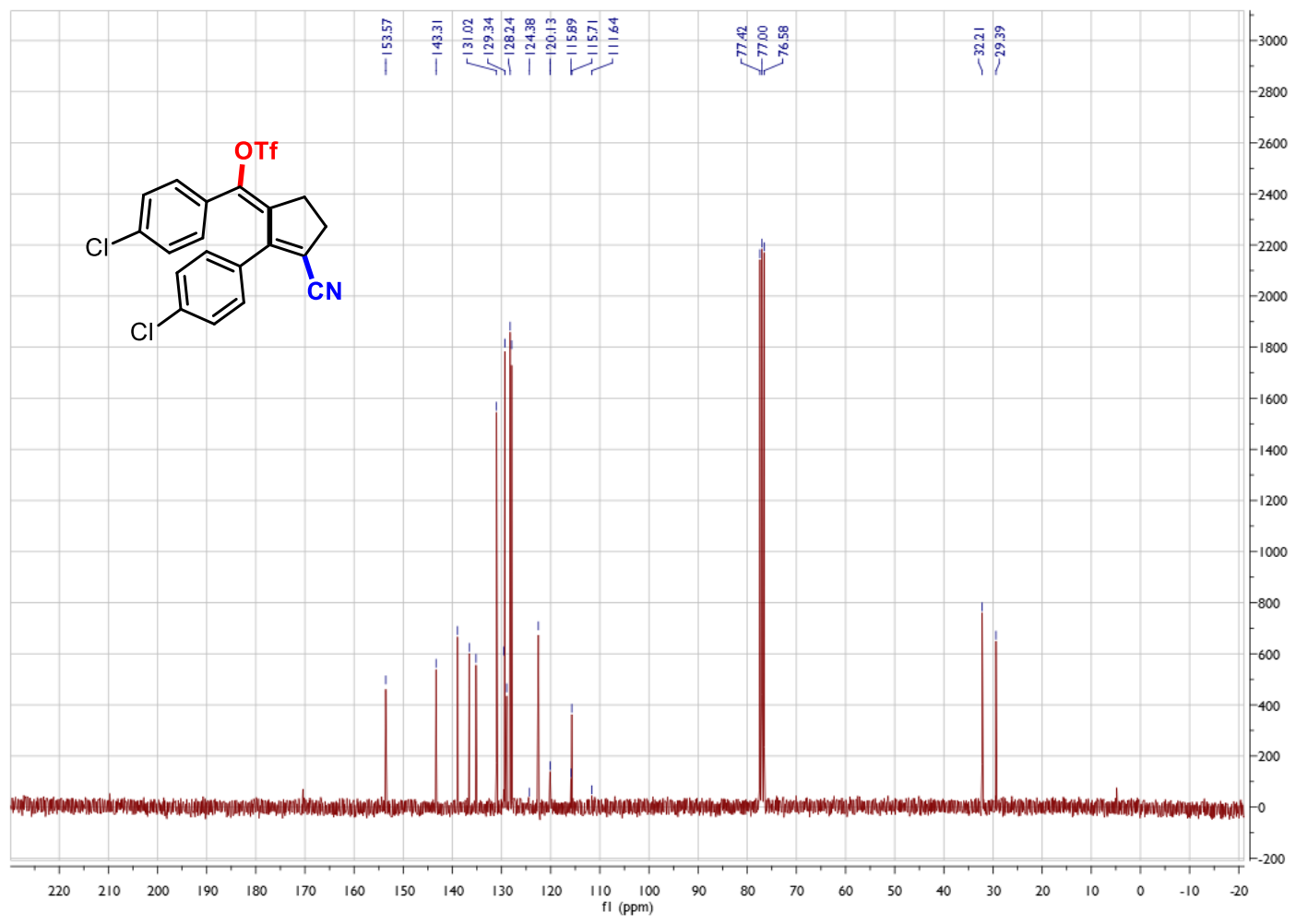


${ }^{19}$ F NMR Spectrum of $(E)$-(4-chlorophenyl)(2-(4-chlorophenyl)-3-cyanocyclopent2-en-1-ylidene)methyl trifluoromethanesulfonate $2 z$

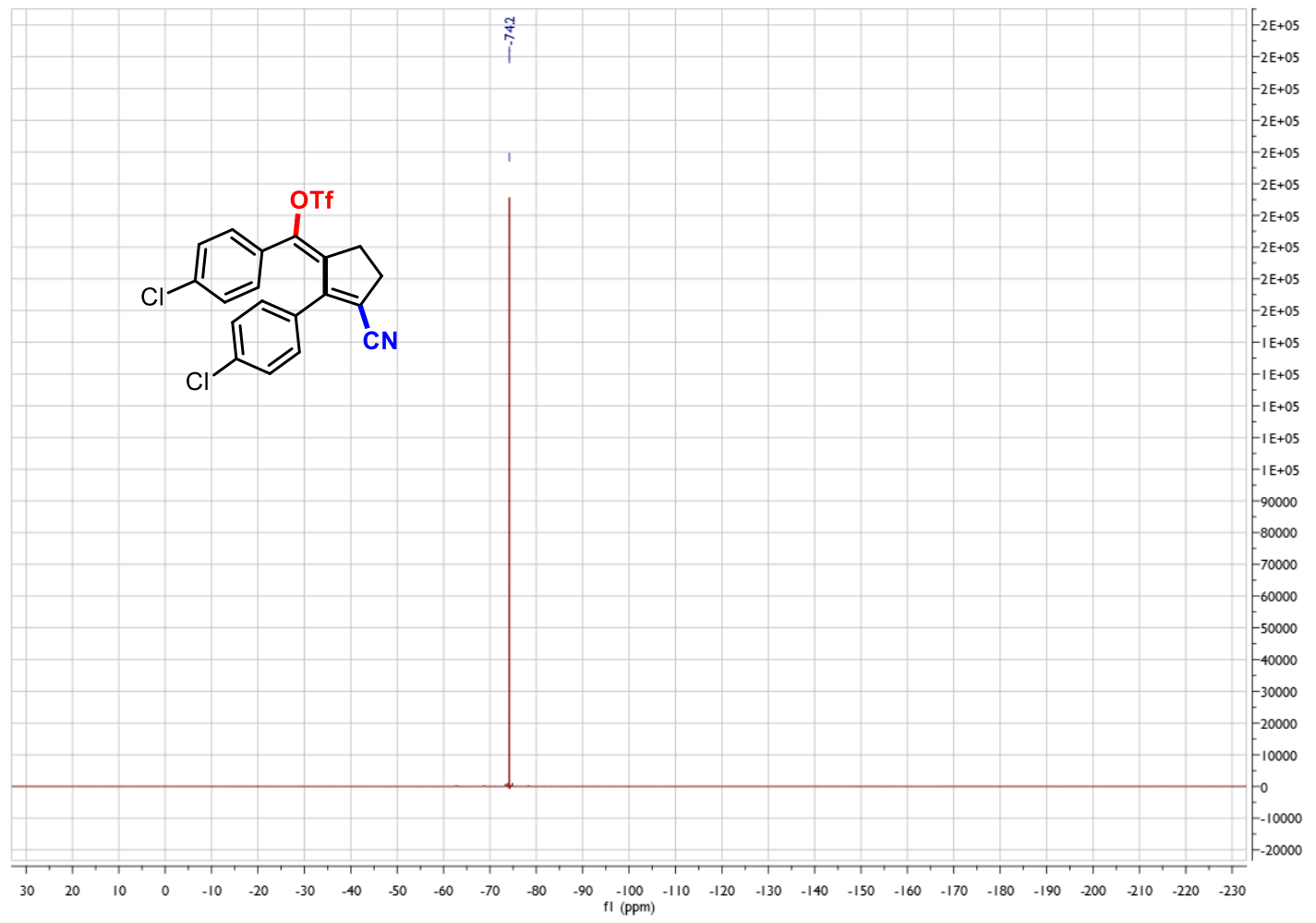

${ }^{19}$ F/1 H-GHOESY Spectrum of $(E)$-(4-chlorophenyl)(2-(4-chlorophenyl)-3cyanocyclo pent-2-en-1-ylidene)methyl trifluoromethanesulfonate $2 \mathrm{z}$

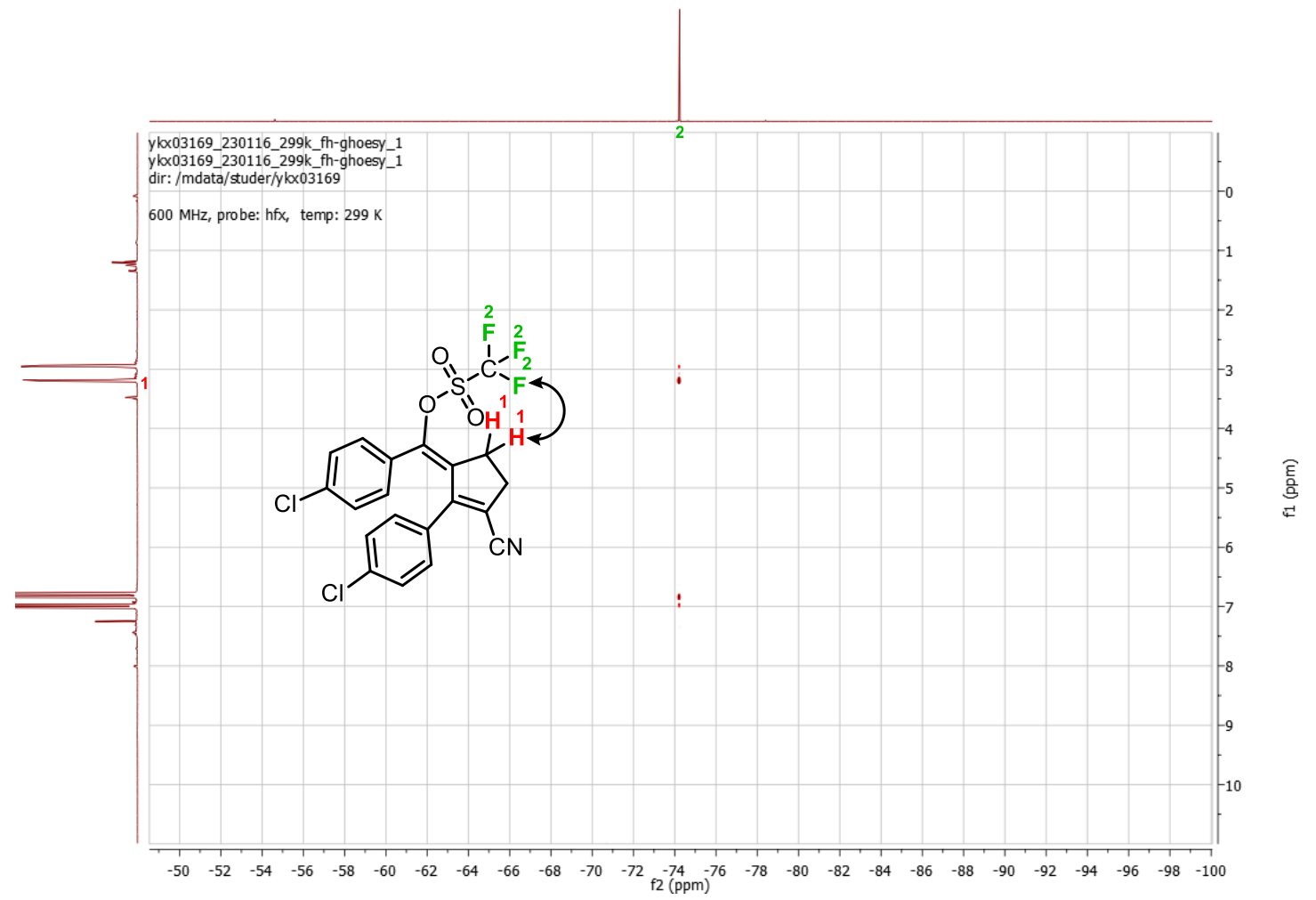


Spectra of products 4-11 in follow-up chemistry

${ }^{1}$ H NMR Spectrum of (Z)-2-((4-methoxyphenyl)(phenyl)methylene)pentanenitrile 4

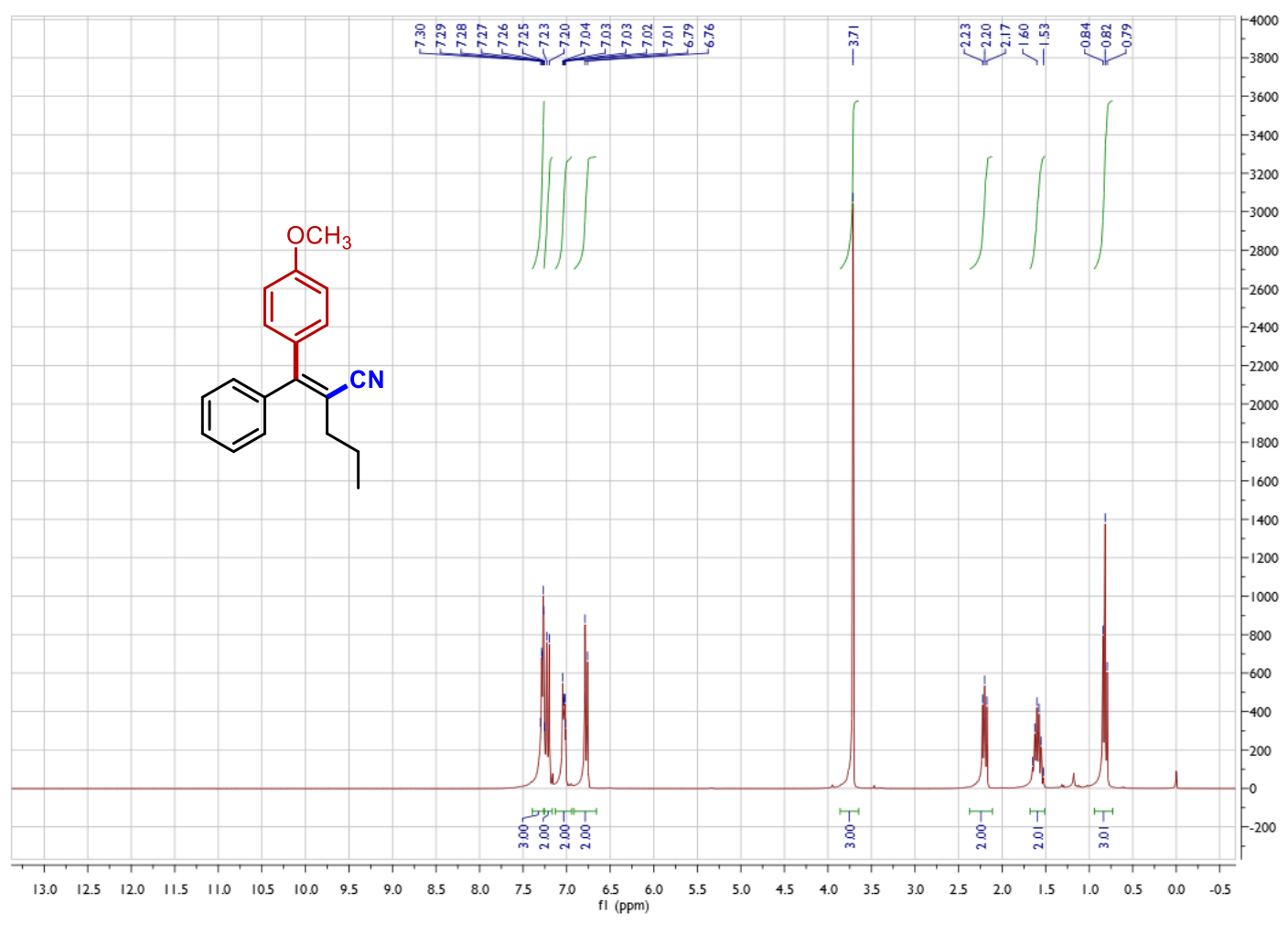

${ }^{13} \mathrm{C}$ NMR Spectrum of (Z)-2-((4-methoxyphenyl)(phenyl)methylene)pentanenitrile 4

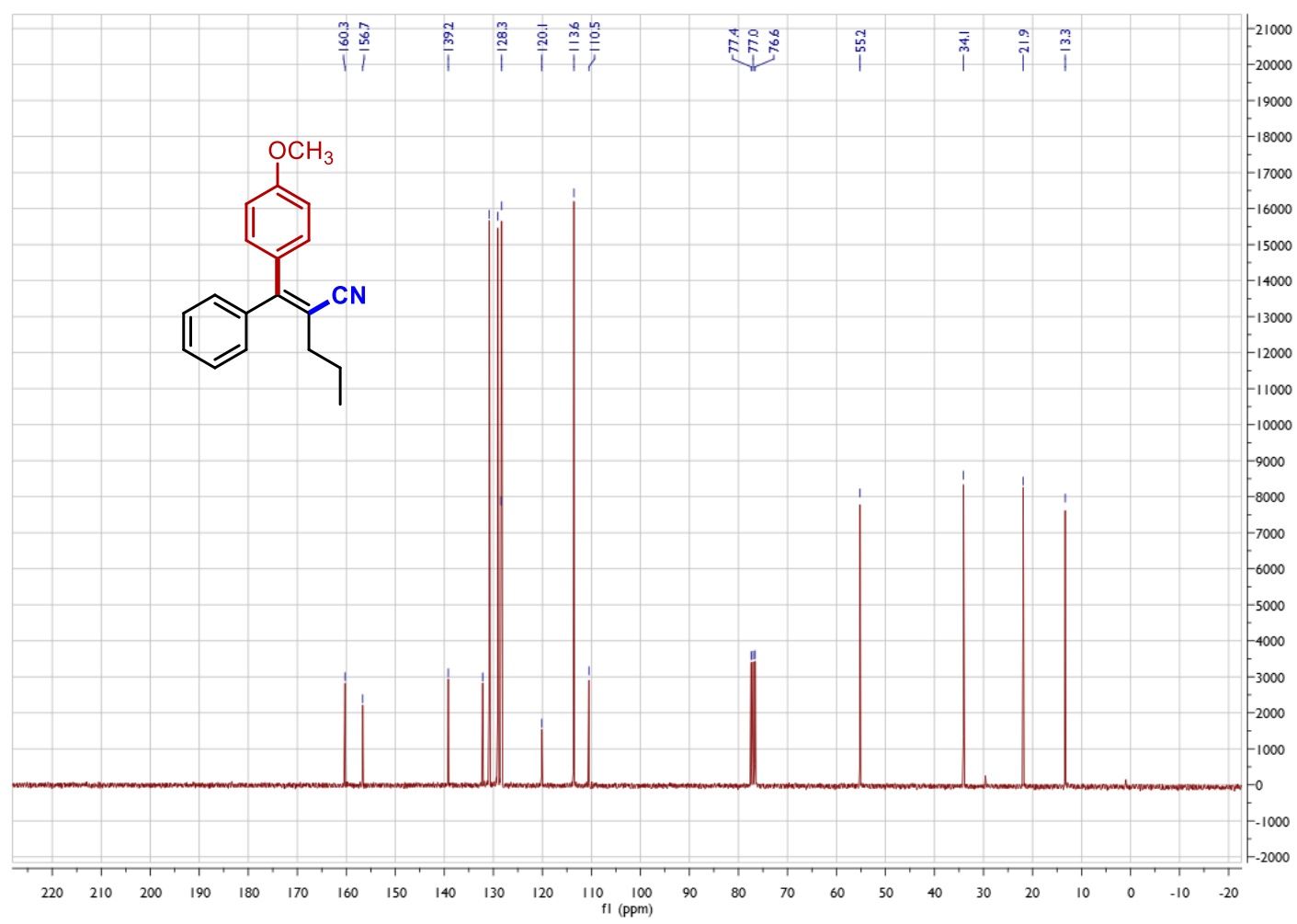


NOESY1D Spectrum of (Z)-2-((4-methoxyphenyl)(phenyl)methylene)pentanenitrile 4

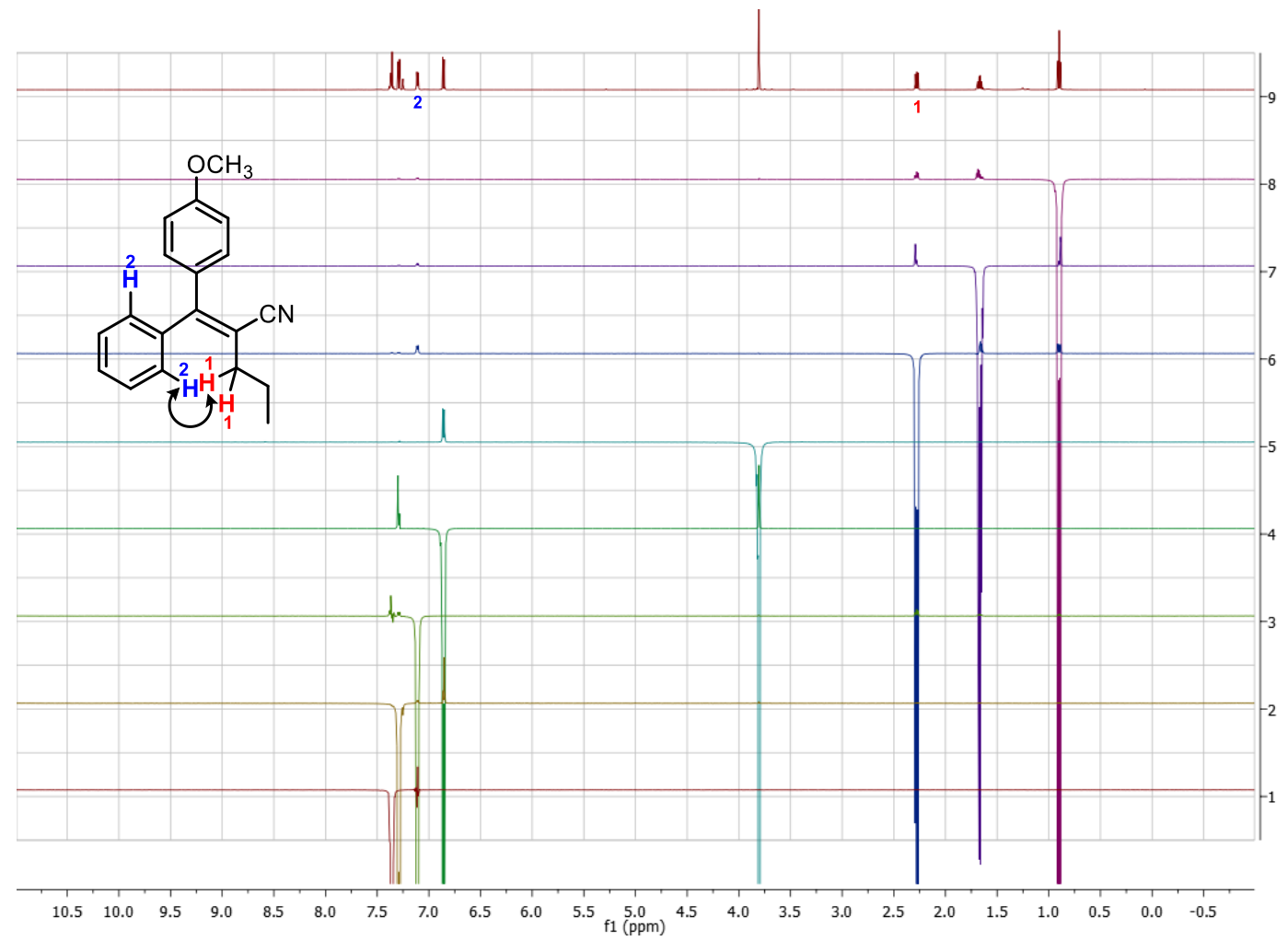


${ }^{1}$ H NMR Spectrum of (E)-3-phenyl-2-propylpenta-2, 4-dienenitrile 5

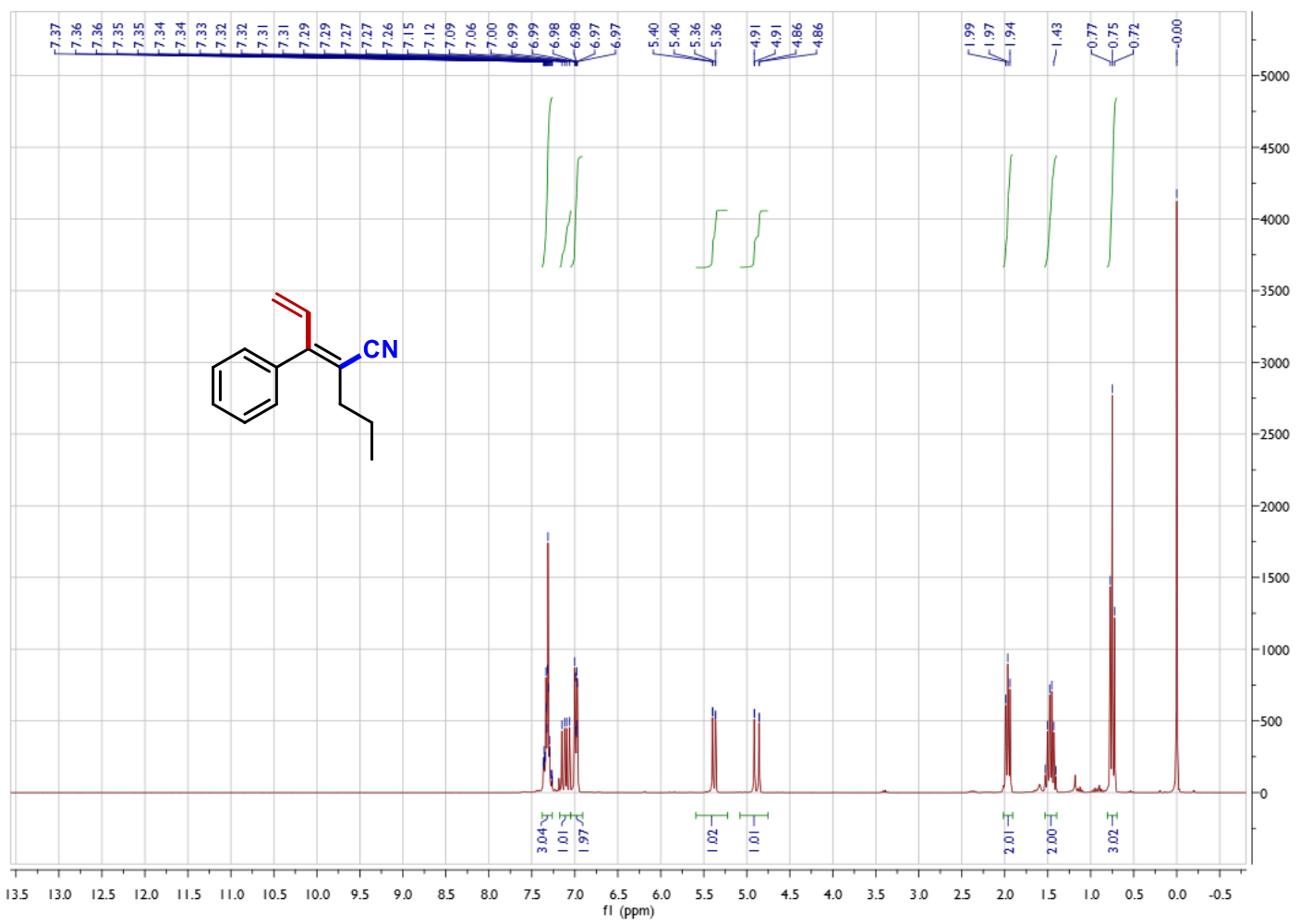

${ }^{13} \mathrm{C}$ NMR Spectrum of $(E)$-3-phenyl-2-propylpenta-2, 4-dienenitrile 5

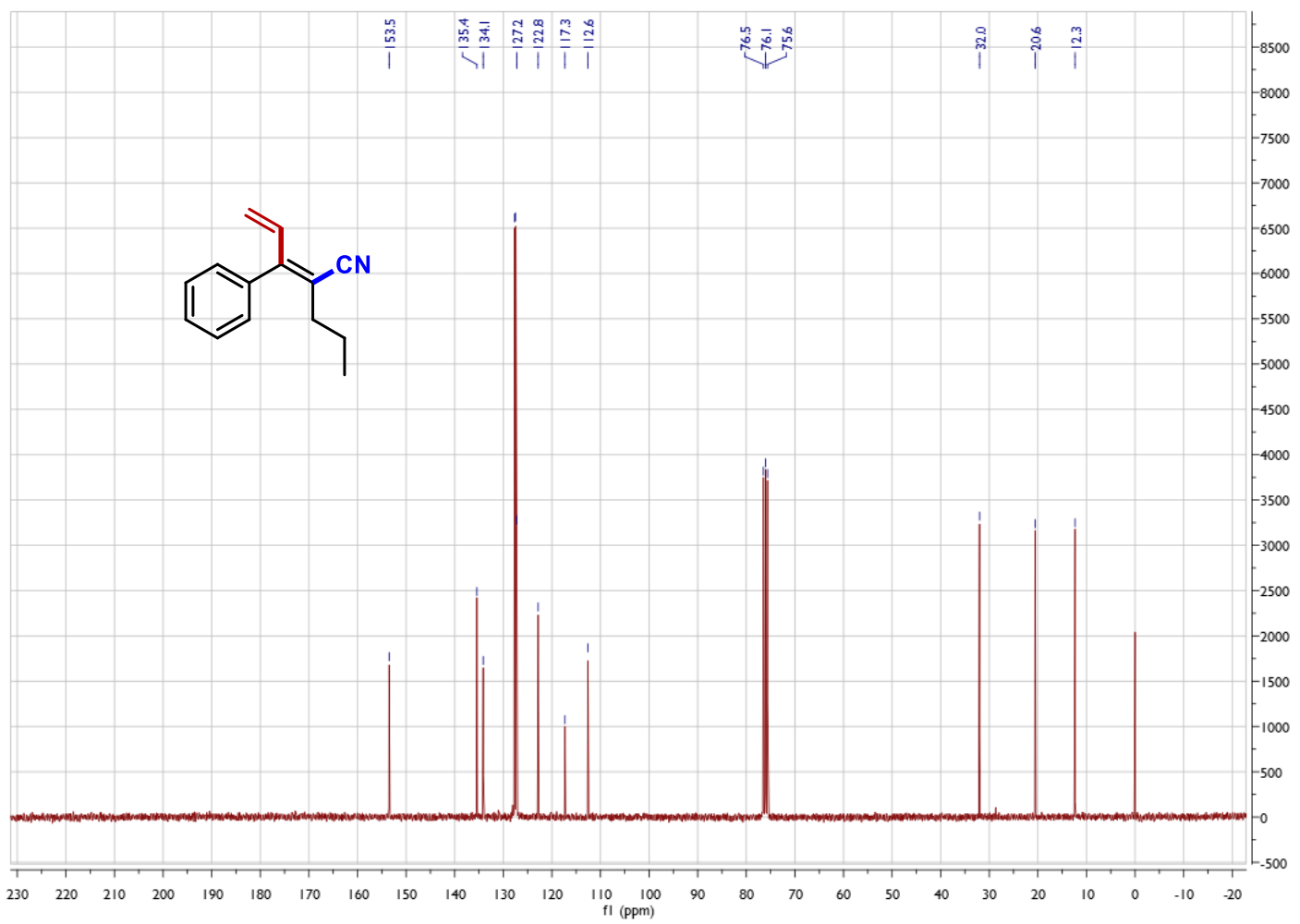


NOESY1D Spectrum of $(E)$-3-phenyl-2-propylpenta-2, 4-dienenitrile 5

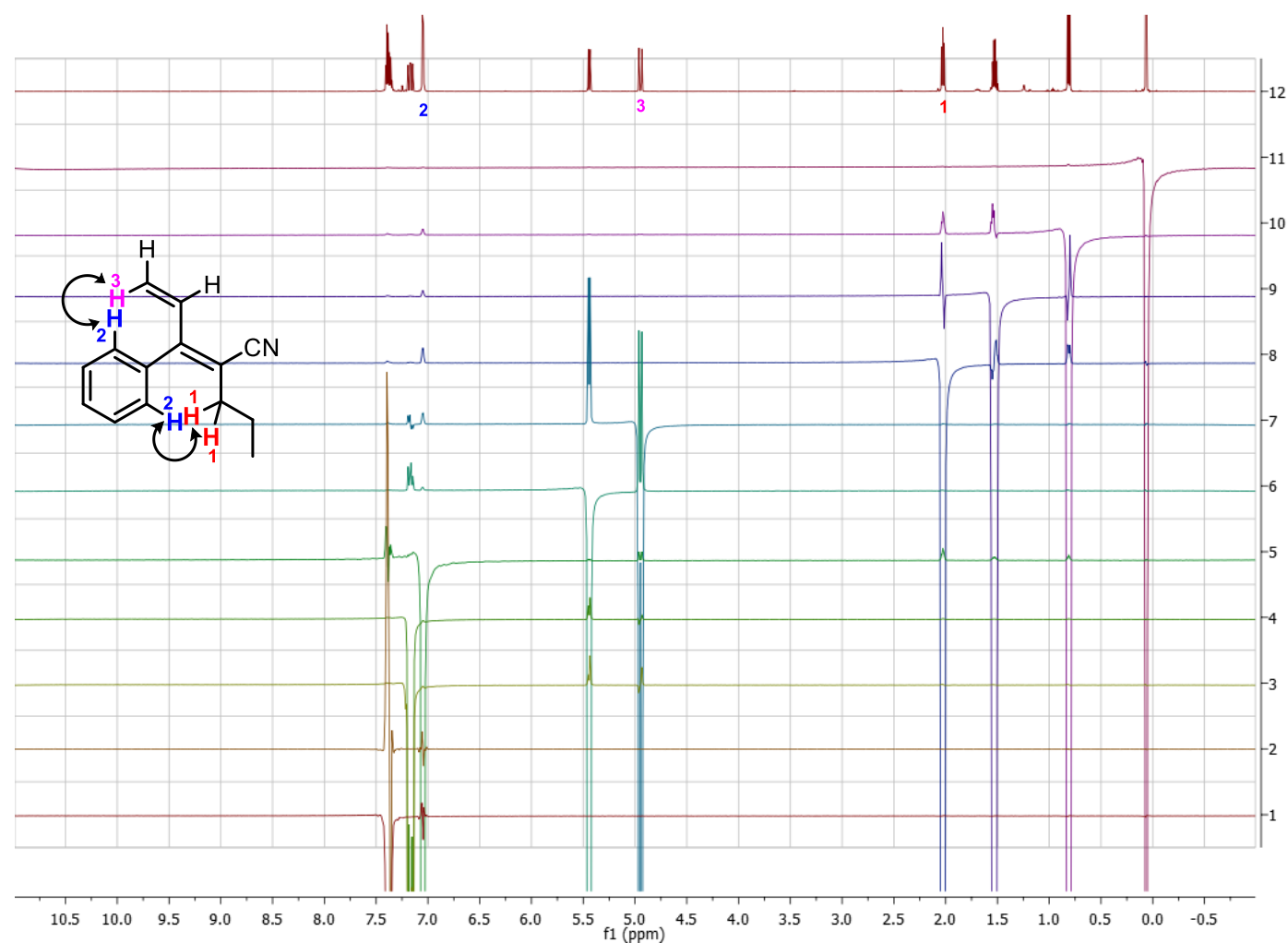


${ }^{1}$ H NMR Spectrum of (Z)-7-hydroxy-3-phenyl-2-propylhept-2-en-4-ynenitrile 6

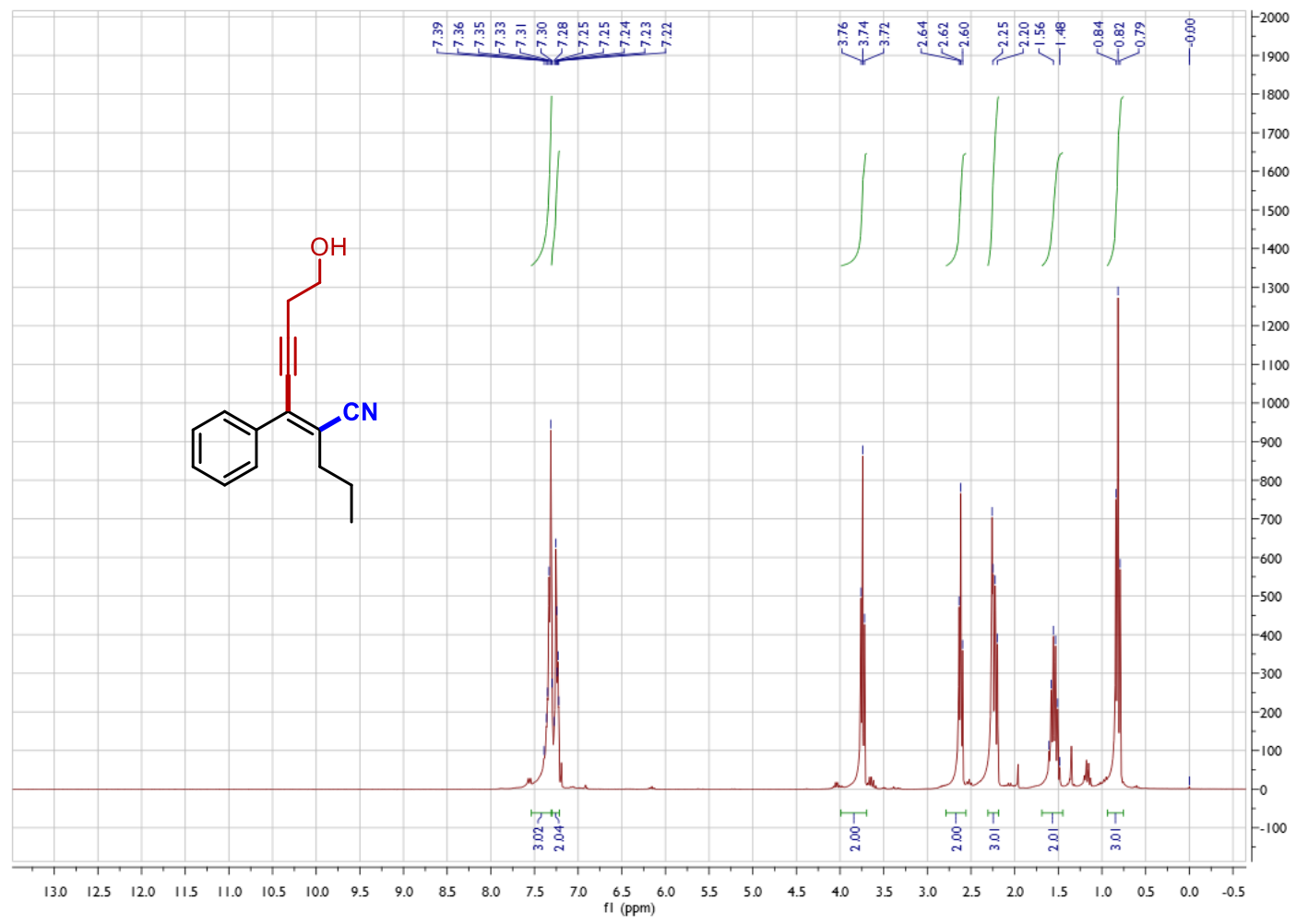

${ }^{13}$ C NMR Spectrum of (Z)-7-hydroxy-3-phenyl-2-propylhept-2-en-4-ynenitrile 6

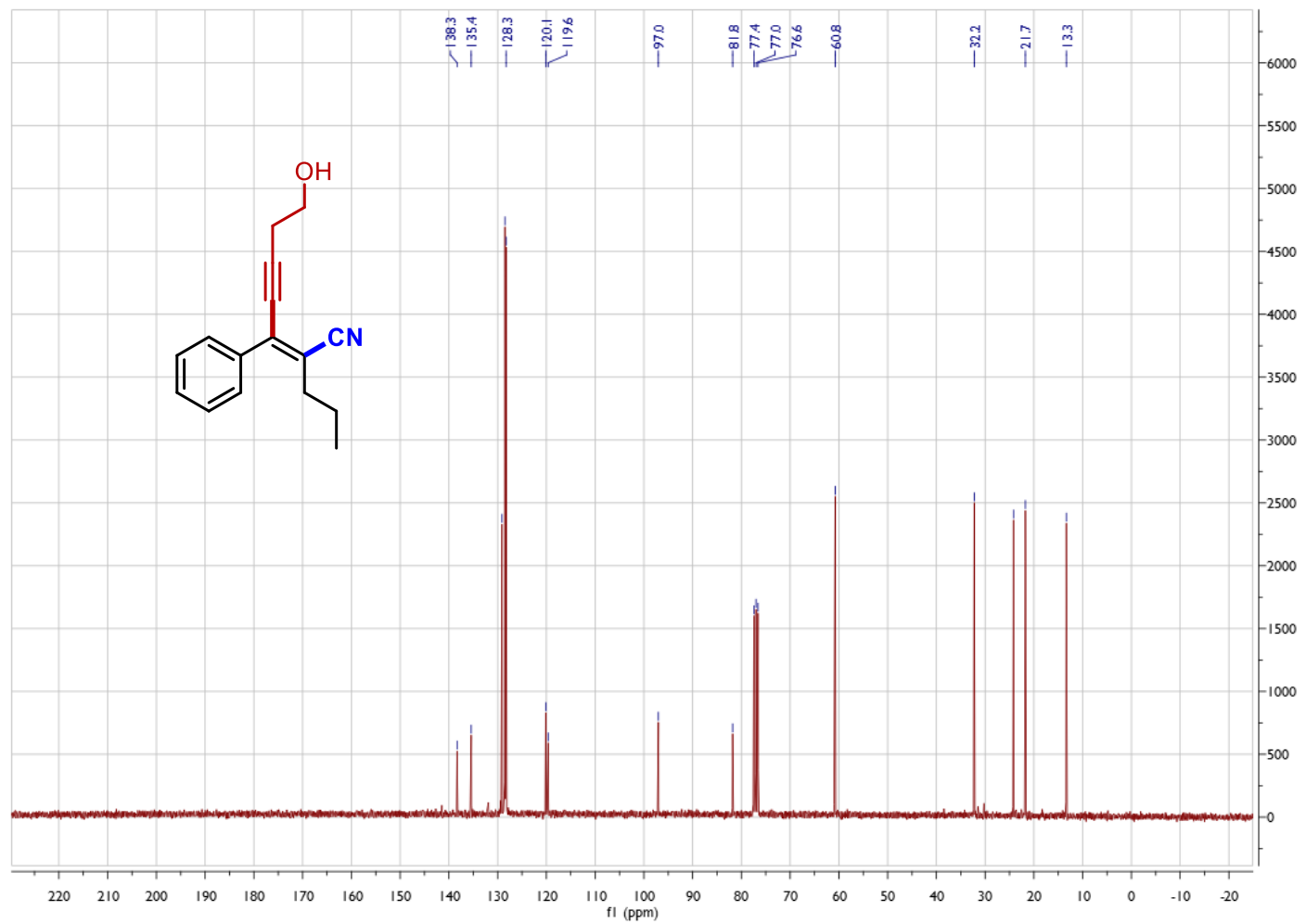


NOESZ1D Spectrum of (Z)-7-hydroxy-3-phenyl-2-propylhept-2-en-4-ynenitrile 6

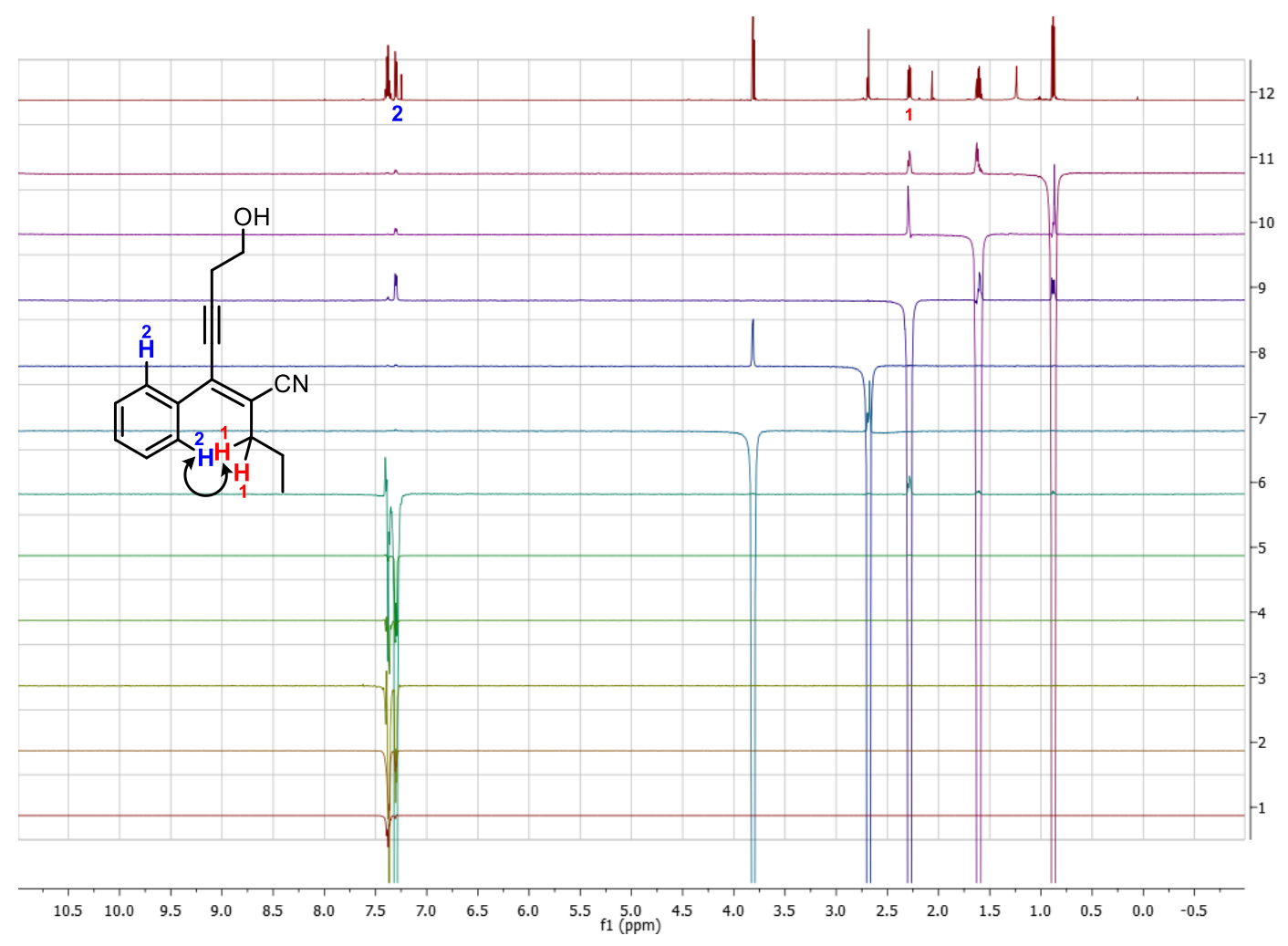


${ }^{1} \mathrm{H}$ NMR Spectrum of $(E)-N$-(2-cyano-1-phenylpent-1-en-1-yl)benzamide 7

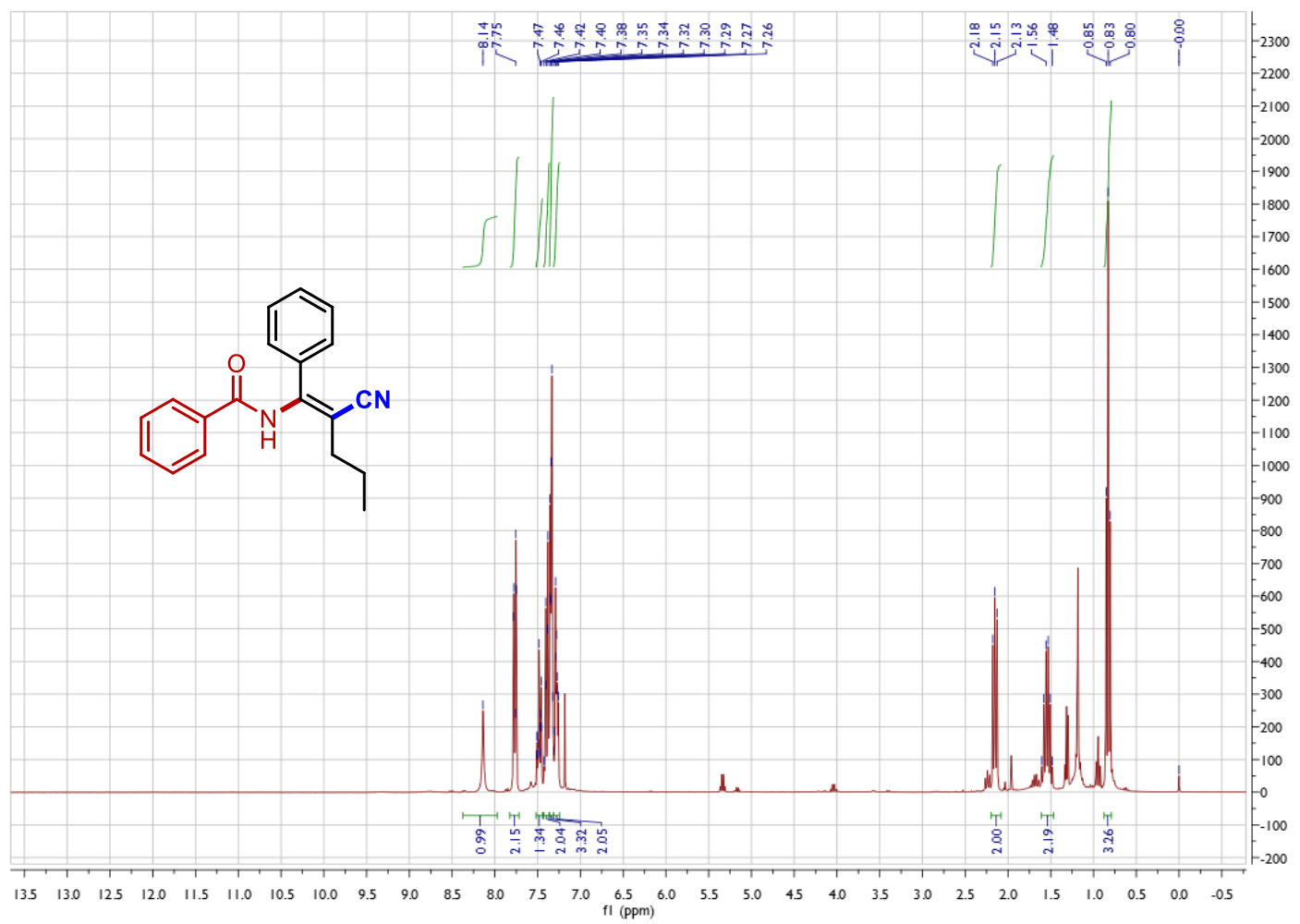

${ }^{13} \mathrm{C}$ NMR Spectrum of $(E)-N$-(2-cyano-1-phenylpent-1-en-1-yl)benzamide 7

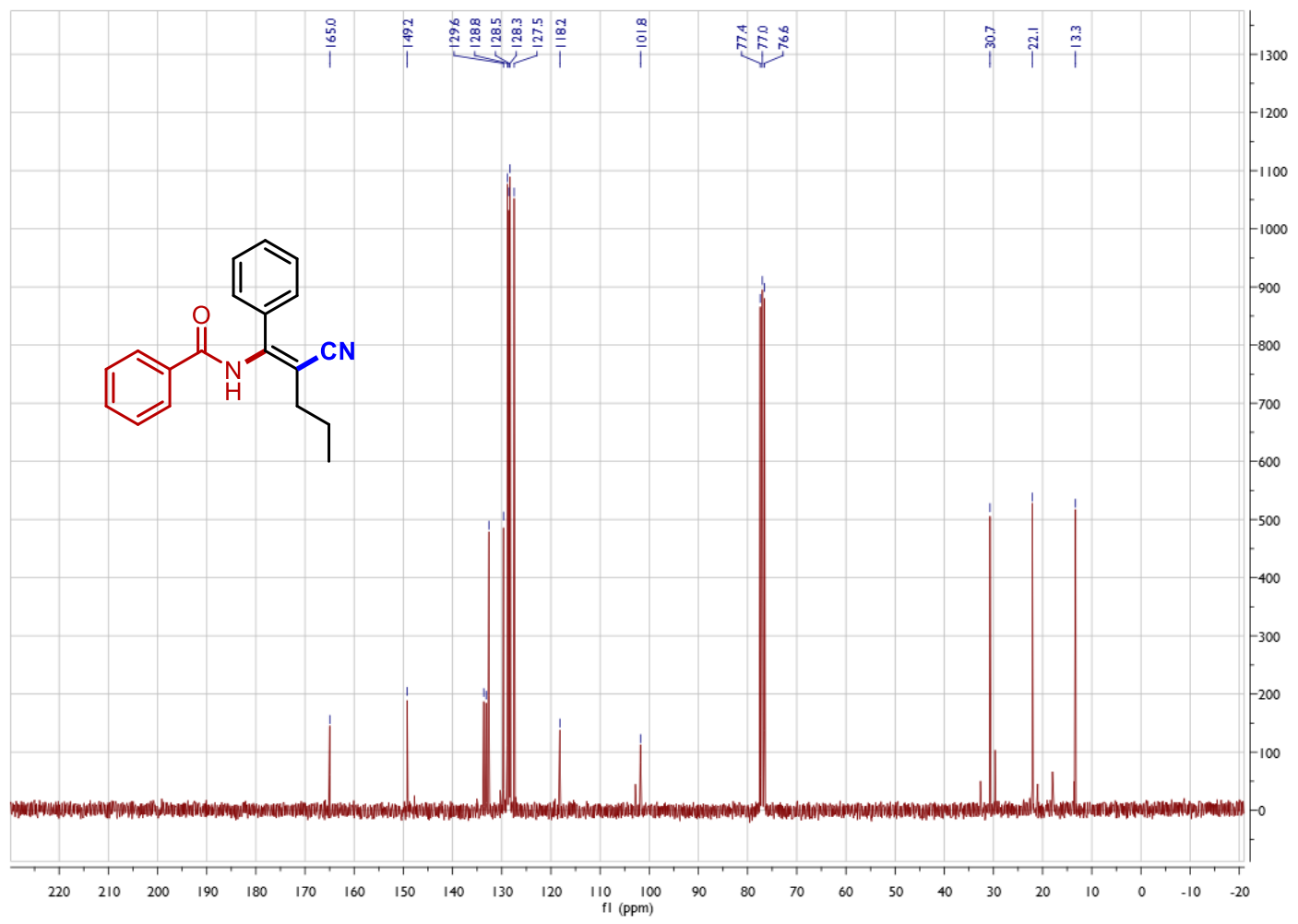


NOESY1D Spectrum of $(E)-N$-(2-cyano-1-phenylpent-1-en-1-yl)benzamide 7

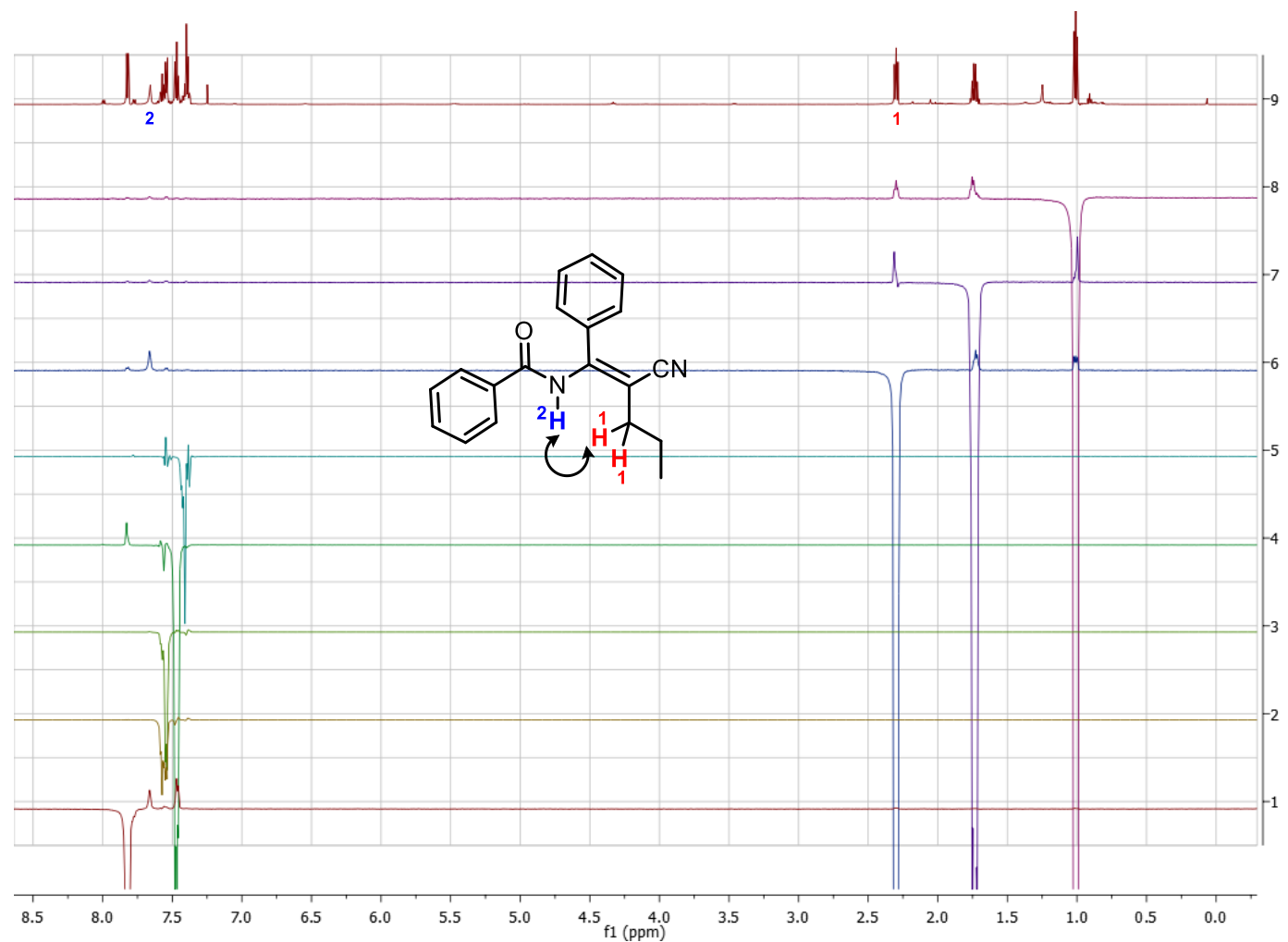


${ }^{1}$ H NMR Spectrum of methyl (Z)-3-cyano-2-phenylhex-2-enoate 8

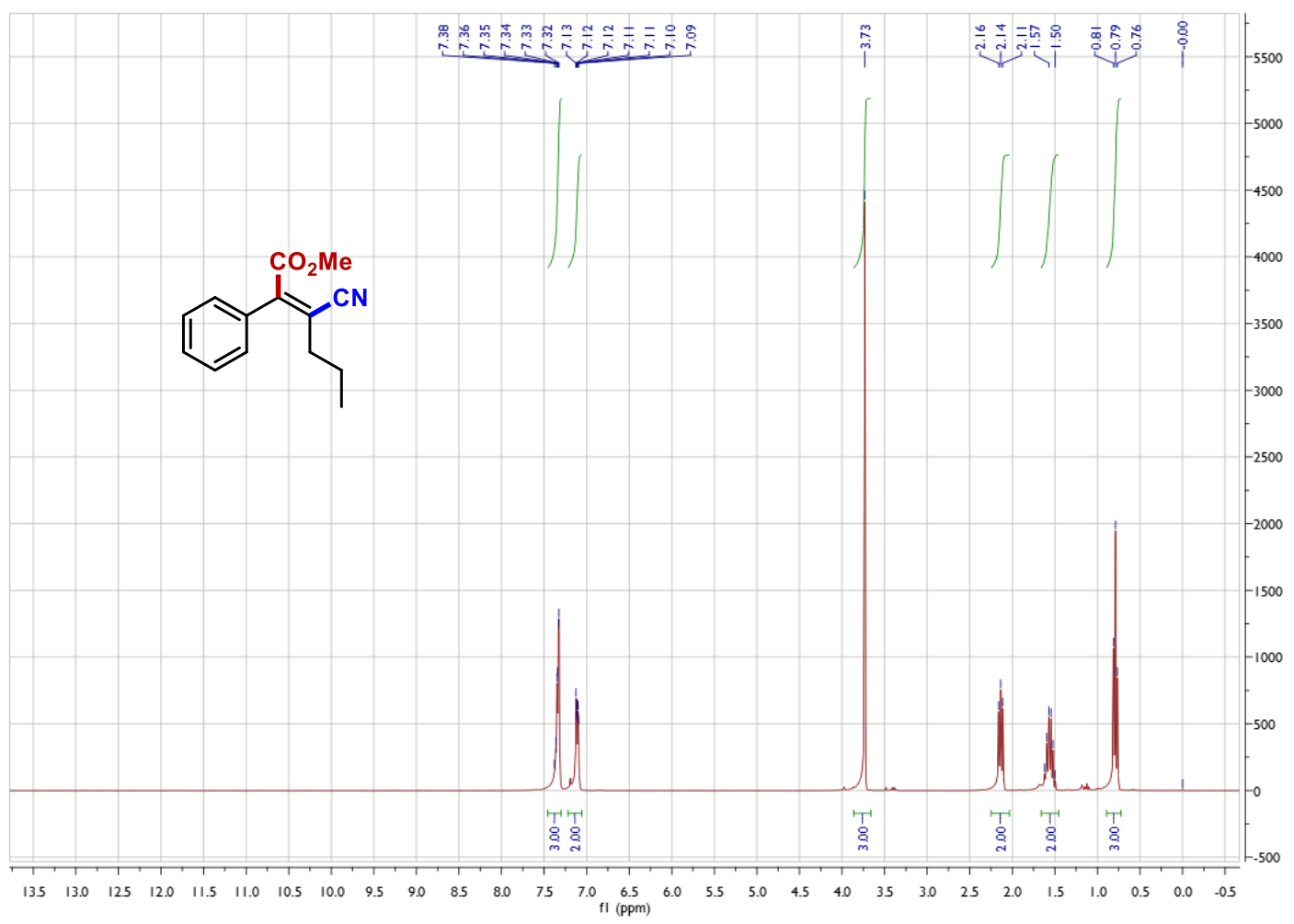

${ }^{13}$ C NMR Spectrum of methyl (Z)-3-cyano-2-phenylhex-2-enoate 8

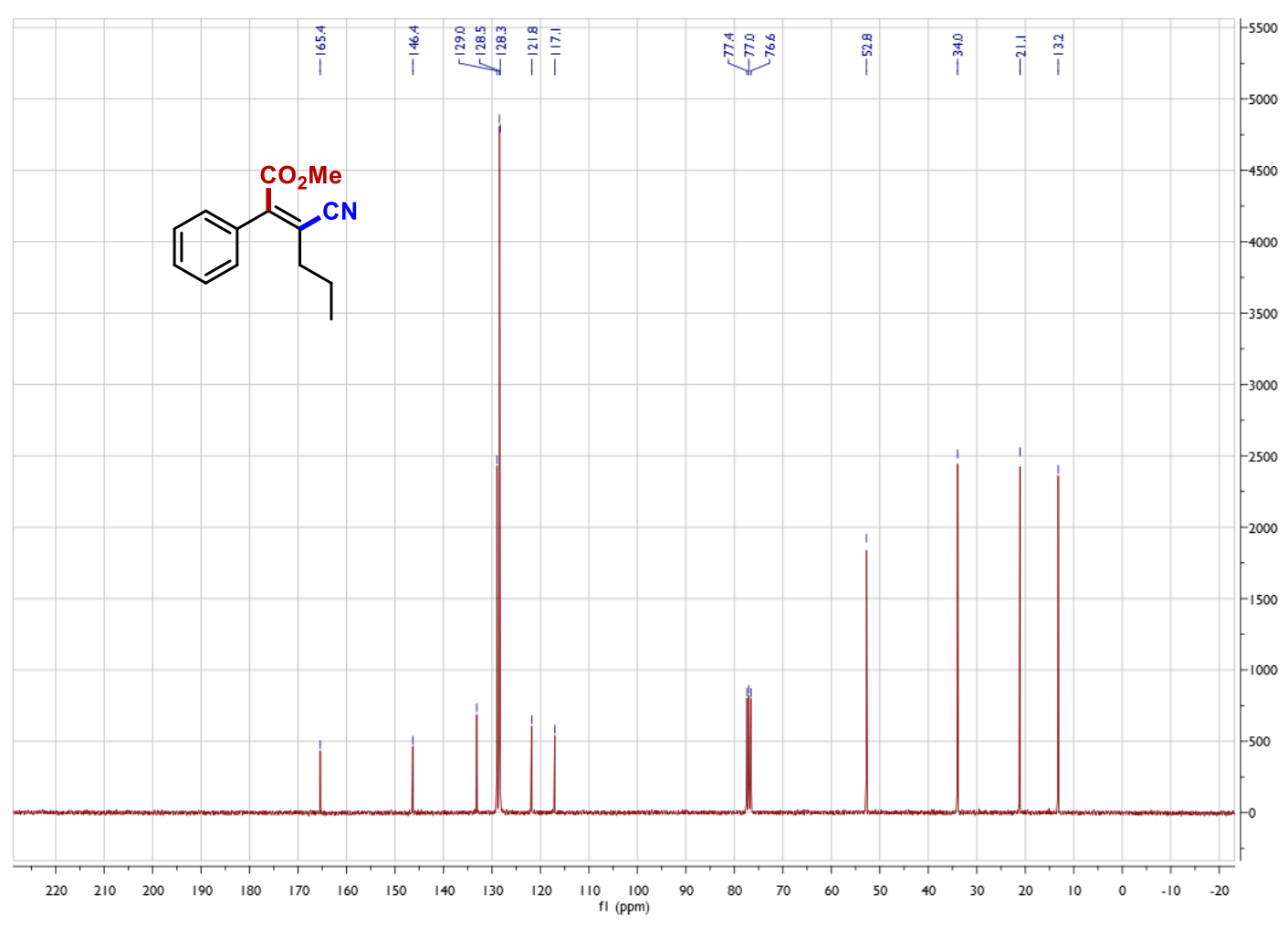


NOESY1D Spectrum of methyl (Z)-3-cyano-2-phenylhex-2-enoate 8

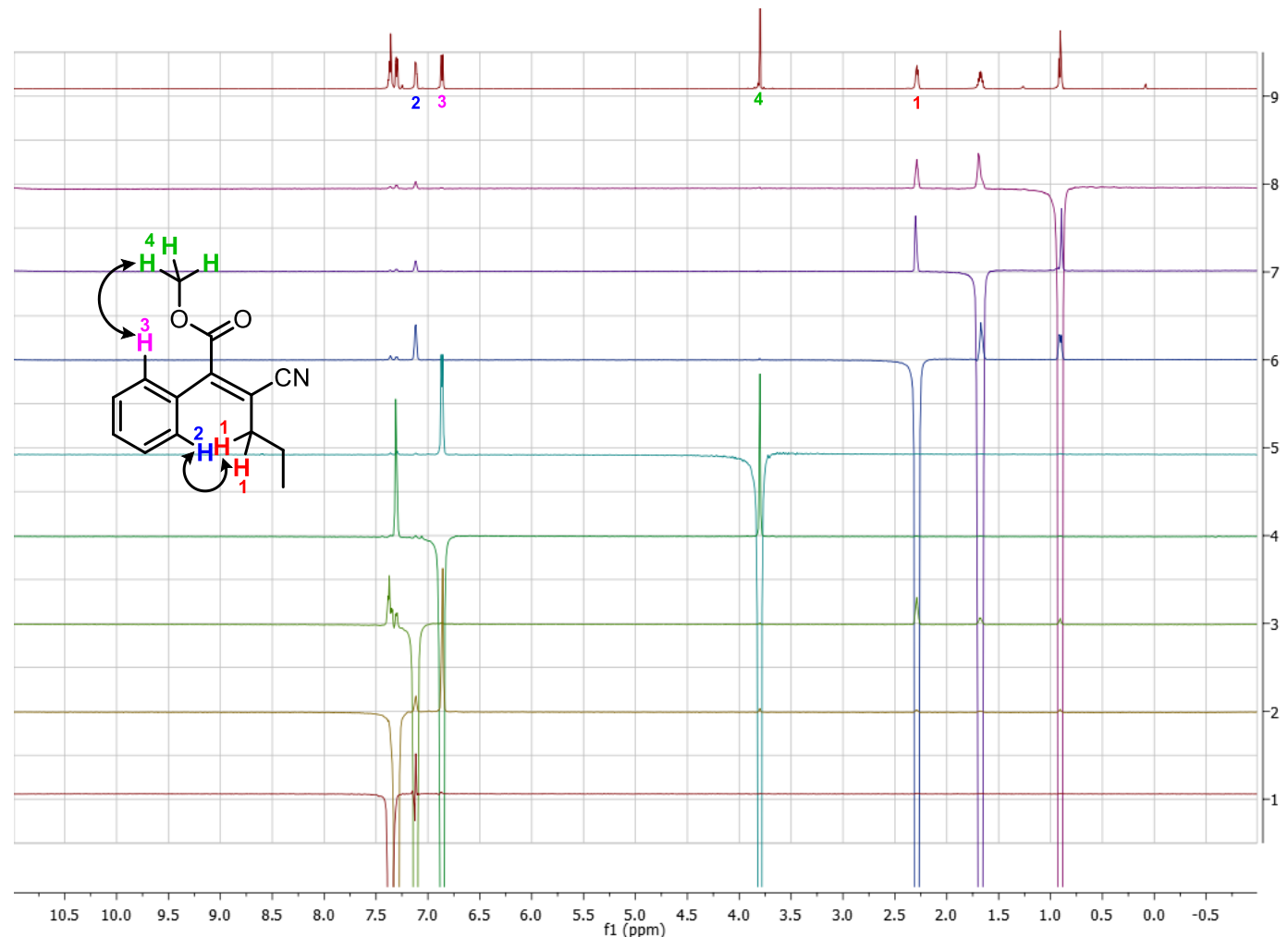


${ }^{1} \mathrm{H}$ NMR Spectrum of 2-benzoylpentanenitrile 9

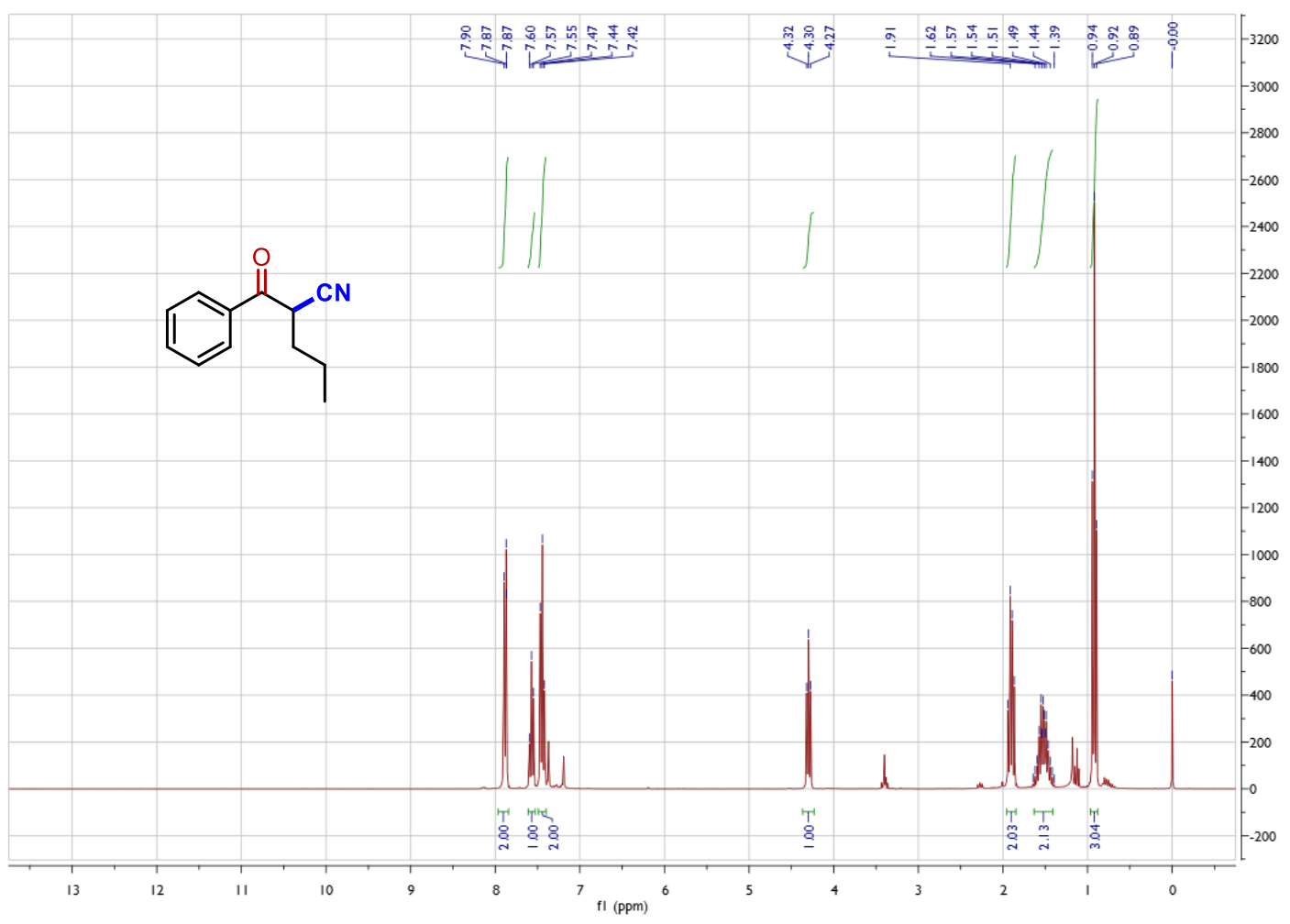

${ }^{13}$ C NMR Spectrum of 2-benzoylpentanenitrile 9

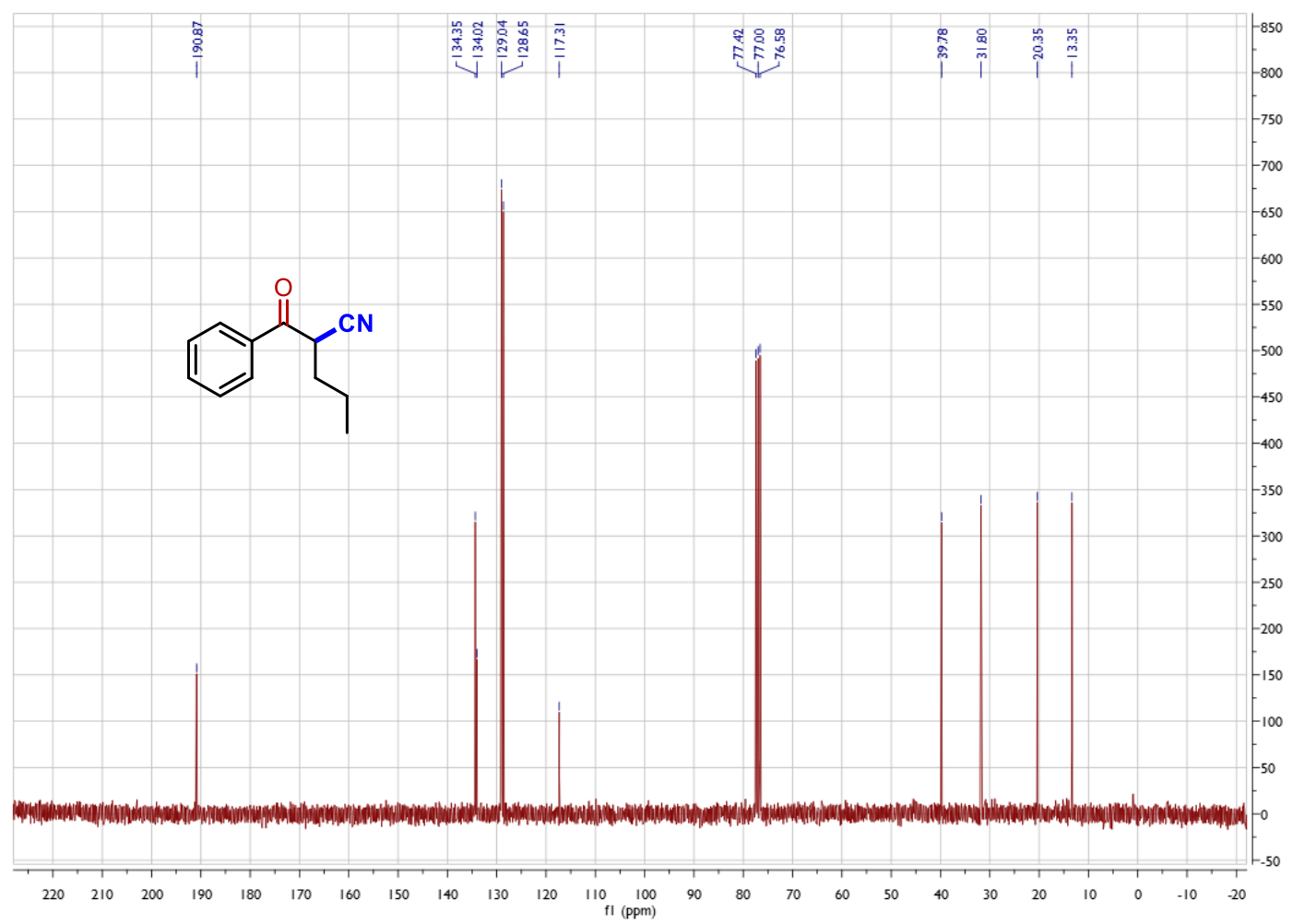


${ }^{1}$ H NMR Spectrum of 2-Benzoylpentanamide 10

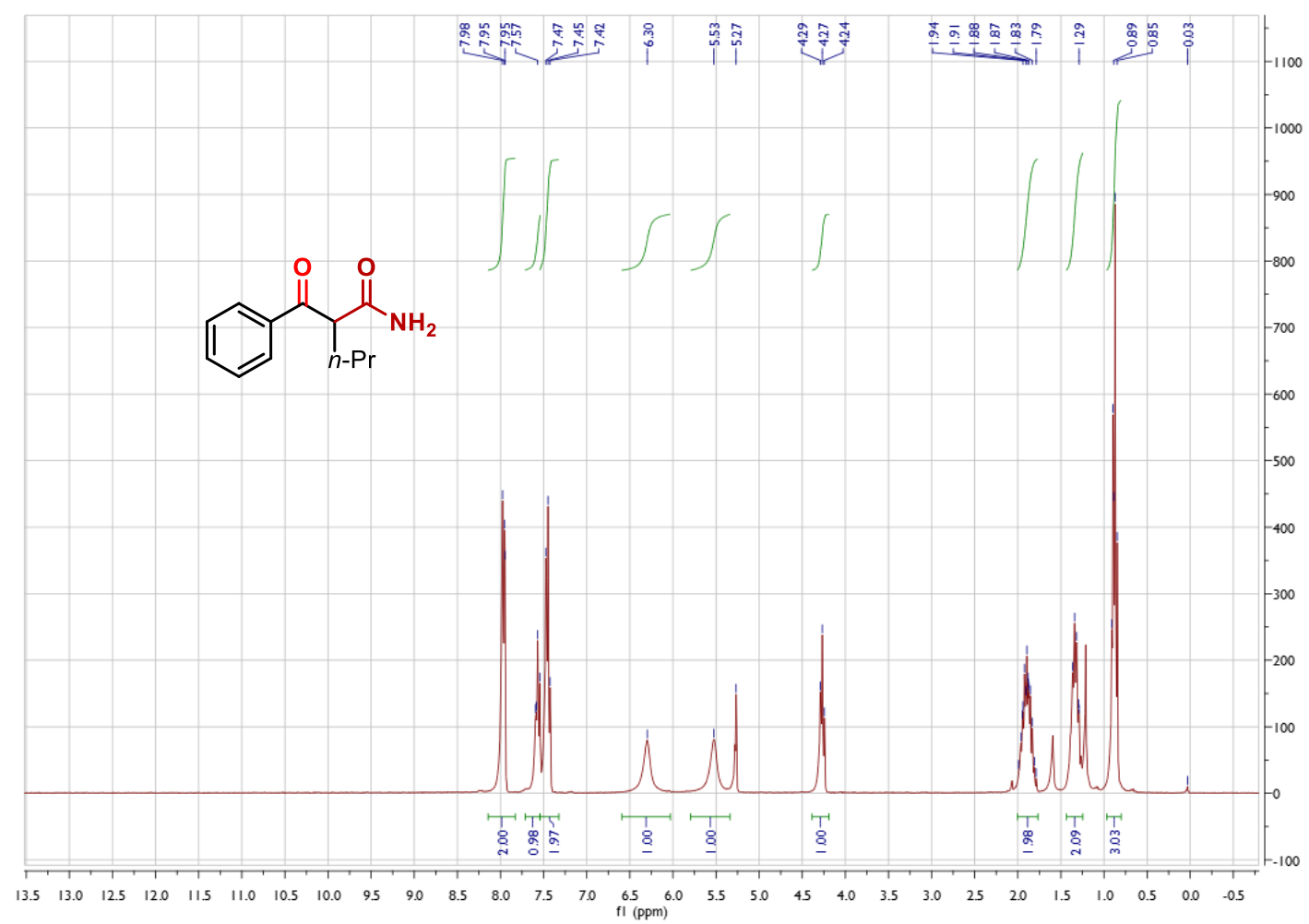

${ }^{13}$ C NMR Spectrum of 2-Benzoylpentanamide 10

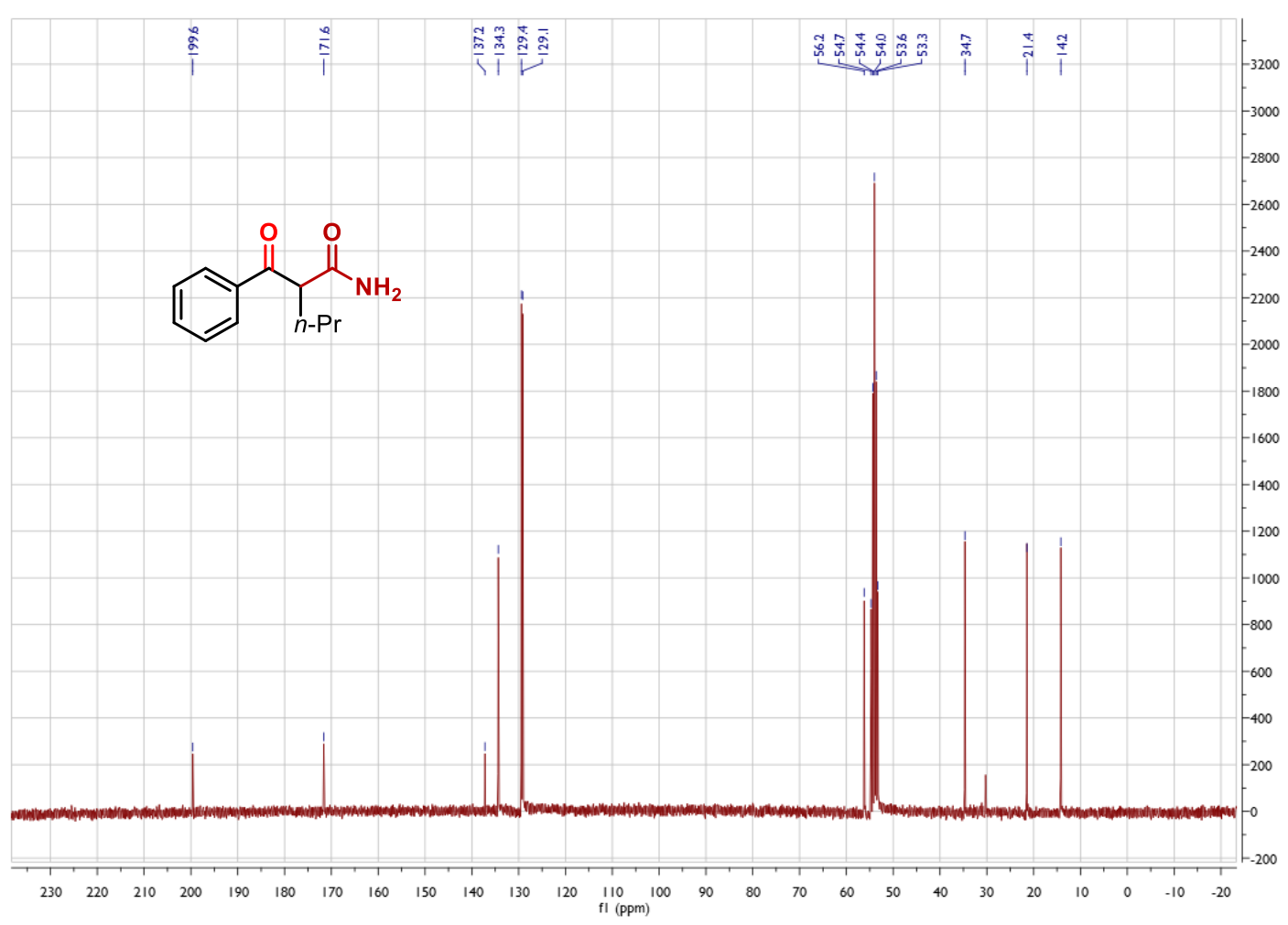


${ }^{1}$ H NMR Spectrum of Ethyl 3-amino-5-phenyl-4-propylthiophene-2-carboxylate 11

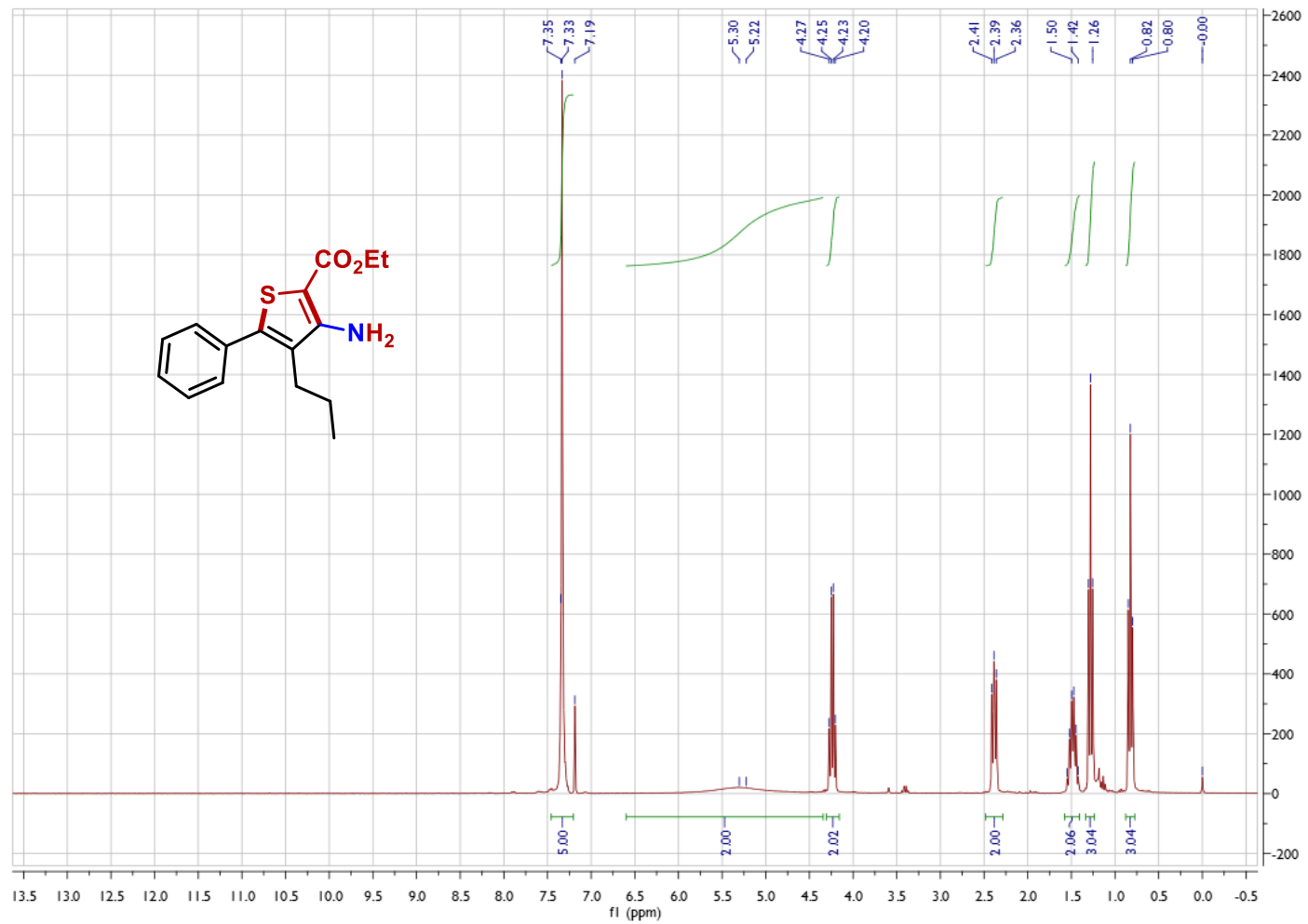

${ }^{13}$ C NMR Spectrum of Ethyl 3-amino-5-phenyl-4-propylthiophene-2-carboxylate 11

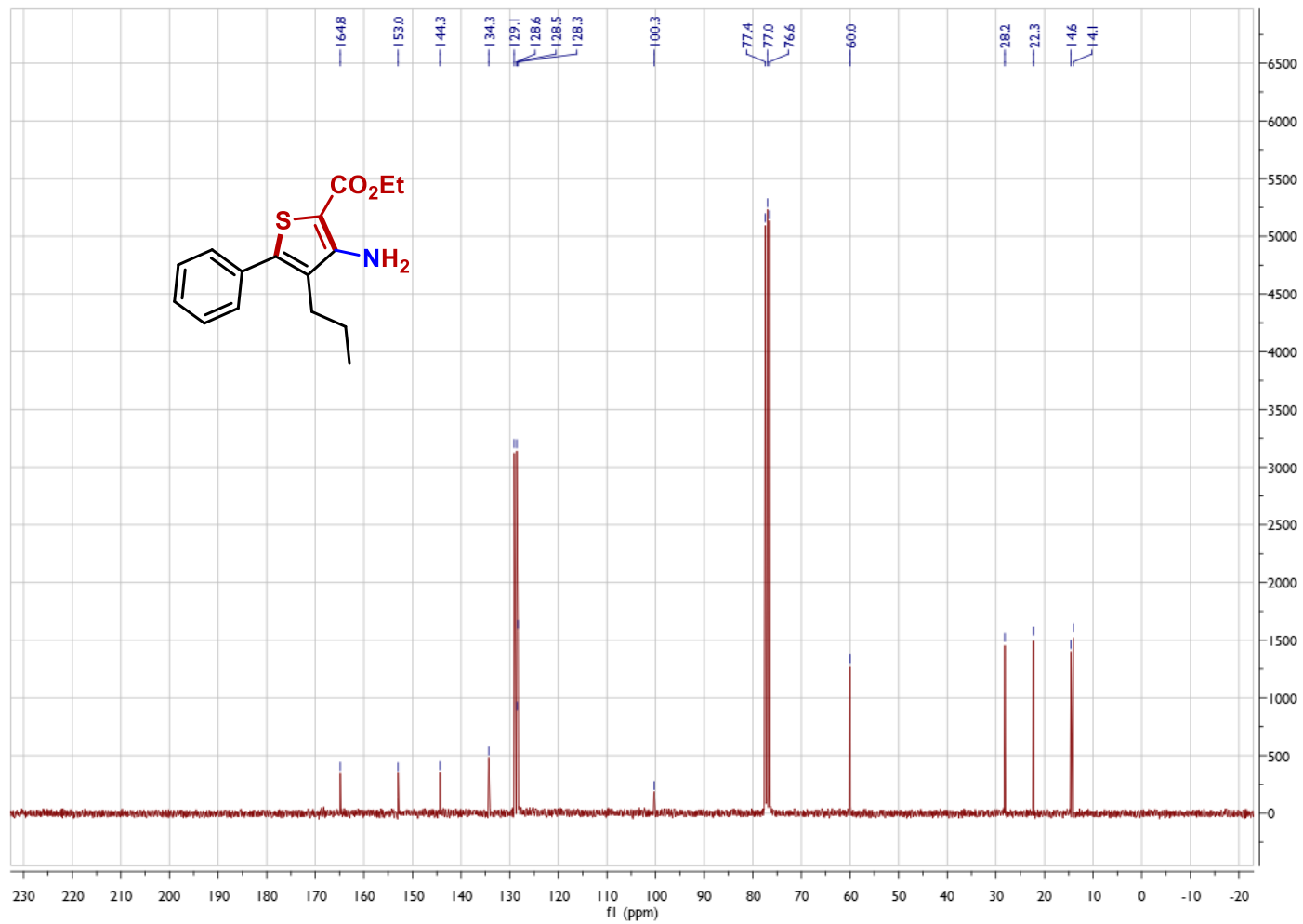




\section{References}

[1] (a) Frei, R.; Courant, T.; Wodrich, M. D.; Waser, J. Chem. Eur. J. 2015, 21, 2662-2668; (b) Zhu, D.; Chang, D.; Shi, L. Chem. Commun. 2015, 51, 7180-7183; (c) Zhdankin, V. V.; Scheuller M. C.; Stang, P. J. Tetrahedron Lett. 1993, 34, 6853-6856.

[2] Songis, O.; Míšek, J.; Schmid, M. B.; Kollárovič, A.; Stará, I. G.; Saman, D.; Císařová, I.; Starý, I. J. Org. Chem. 2010, 75, 6889-6899.

[3] Miersch, A.; Hilt, G. Chem. Eur. J. 2012, 18, 9798-9801.

[4] Tong, L.; Qin, A.; Zhang, X.; Mao, Y.; Sun, J.; Tang, B. Z. Science China: Chemistry, 2011, 54, 1948-1954.

[5] Siebeneicher, H.; Doye, S. Eur. J. Org. Chem. 2002, 1213-1220.

[6] Wang, S.; Yu, L.; Li, P.; Meng, L.; Wang, L. Synthesis 2011, 10, 1541-1546.

[7] Chen, Z.; Jiang, H.; Li, Y.; Qi, C. Chem. Commun. 2010, 46, 8049-8051.

[8] Li, X.; Peng, F.; Zhou, M.; Mo, M.; Zhao, R.; Shao Z. Chem. Commun. 2014, 50, 1745-1747.

[9] You, H.-W.; Lee, K.-J. Synlett 2001, 1, 105-107. 